\title{
Evaluation über die Effektivität eines strukturierten Trainingsprogramms zur Behandlung des chronischen Fatigue-Syndroms bei Brustkrebspatientinnen
}

\author{
Dissertation \\ zur Erlangung des Doktorgrades \\ der Sozialwissenschaftlichen Fakultät \\ der Georg-August-Universität Göttingen
}

vorgelegt von

Marcus Wuttke

aus Göttingen

Göttingen 2010 
1. Gutachter: Prof. Dr. med. Dr. rer. nat. Andree Niklas

2. Gutachter: Prof. Dr. med. Manfred E. Heim

3. Gutachter: Prof. Dr. Arnd Krüger

Tag der mündlichen Prüfung: 
Die vorliegende Studie wurde finanziell gefördert durch die Deutsche Krebshilfe im Rahmen des Förderschwerpunktprogramms Psychosoziale Onkologie. 


\section{Inhalt}

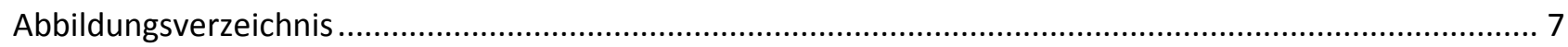

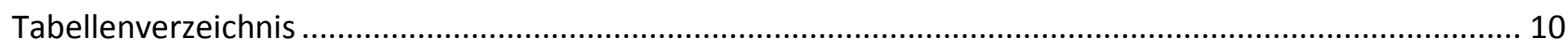

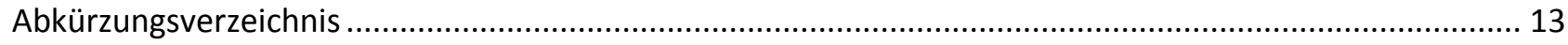

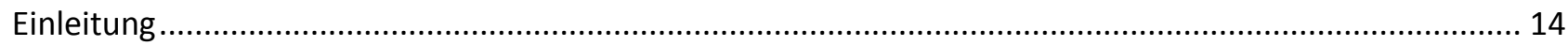

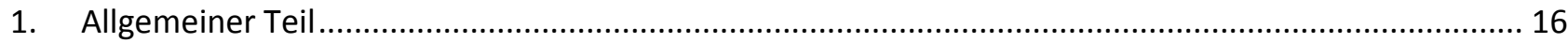

$1.1 \quad$ Funktionelle Beeinträchtigungen nach Brustkrebstherapie....................................................16

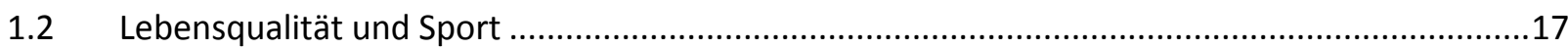

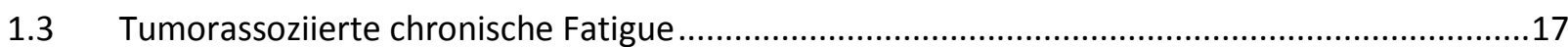

1.3.1 Instrumente zur Erfassung der tumorbedingten Fatigue .....................................................18

1.4 Rehabilitationstraining und medizinische Trainingstherapie ...................................................20

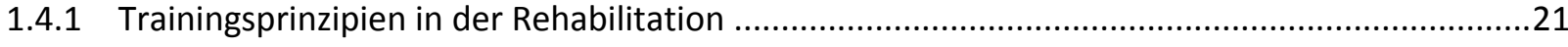

1.4.2 Prinzip der Entwicklungs- und Gesundheitsförderung ...............................................................23

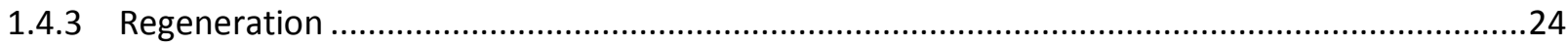

1.5 Psychische und psychophysiologische Einflüsse des Rehabilitationstrainings.............................25

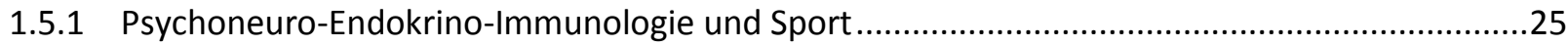

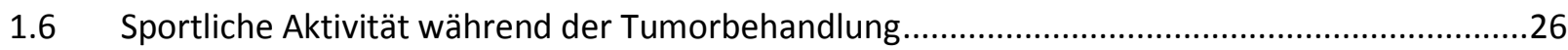

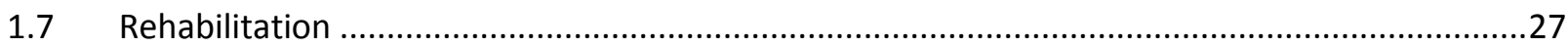

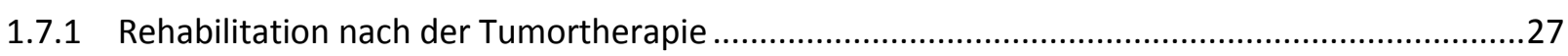

1.8 Krebsnachsorge und ambulante Krebssportgruppen............................................................28

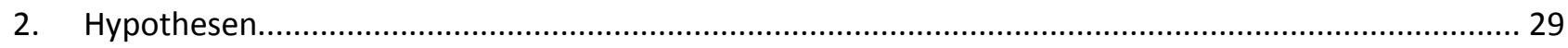

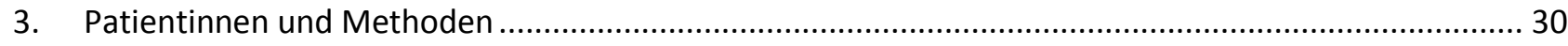

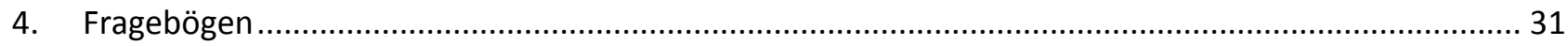

4.1 Fatigue - LASA (Linear-Analog-Self-Assessment-Scale) ............................................................

4.2 HADS (Hospital Anxiety and Depression Scale) ........................................................................

4.3 MFI (Multidimensional Fatigue Inventory) ..............................................................................

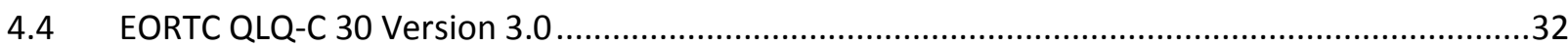

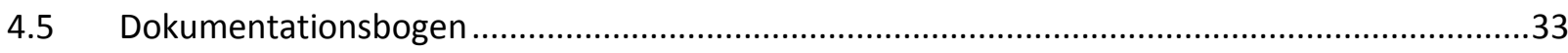

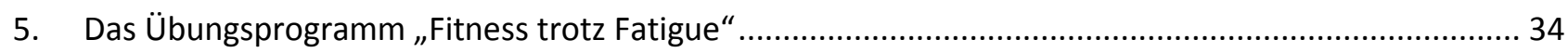

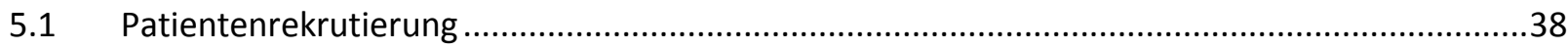

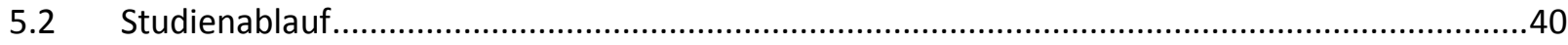

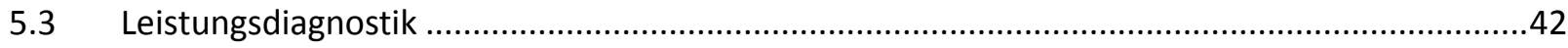

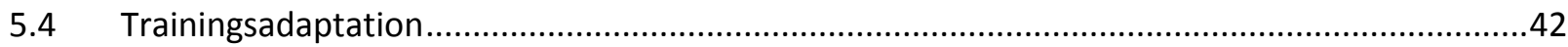




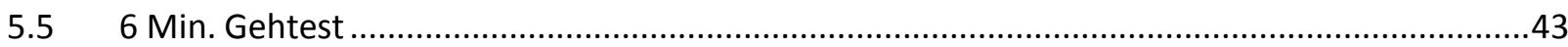

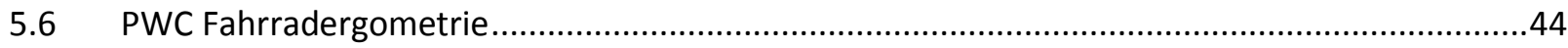

5.7 Messung der Muskelkraft .....................................................................................46

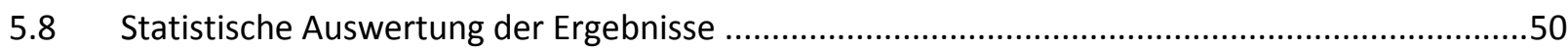

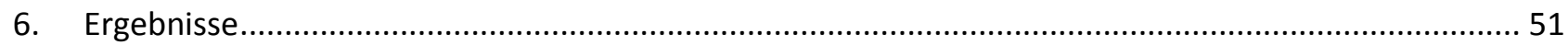

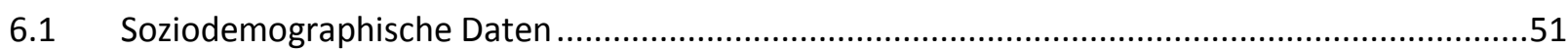

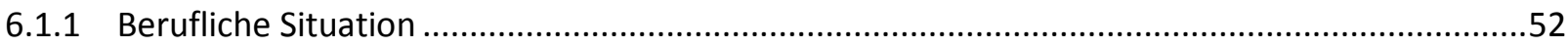

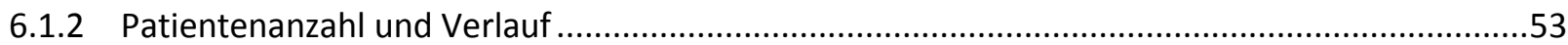

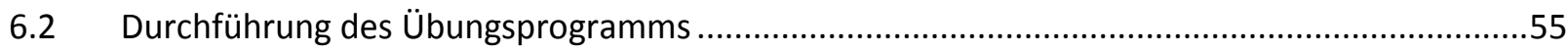

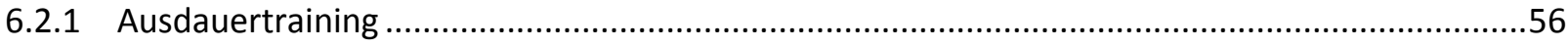

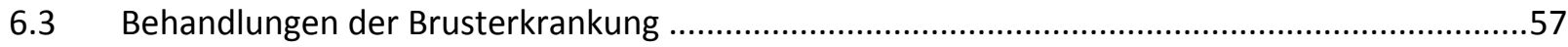

6.3.1 Physiotherapeutische Fragestellungen ...................................................................66

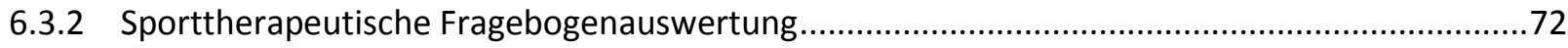

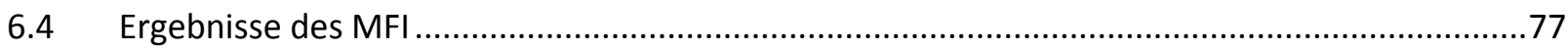

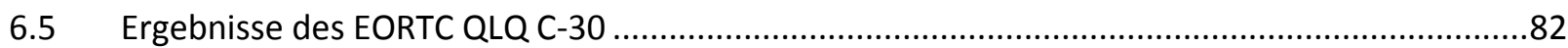

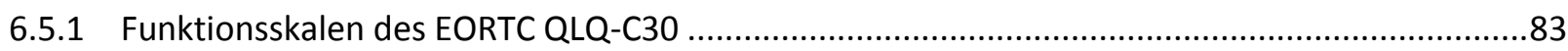

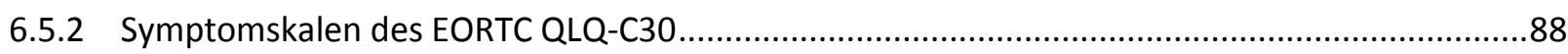

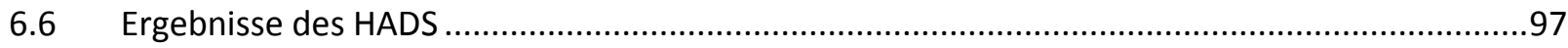

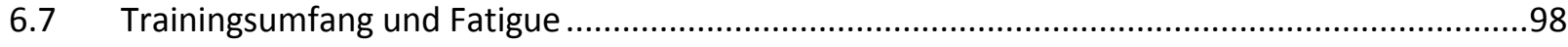

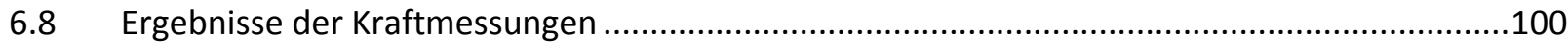

E.9 Ergebnisse der Leistungsdiagnostik.................................................................... 101

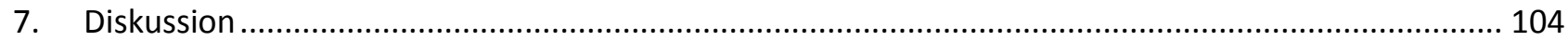

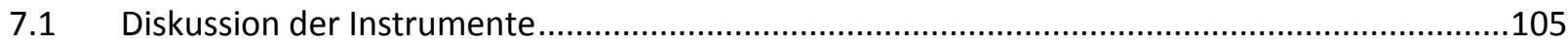

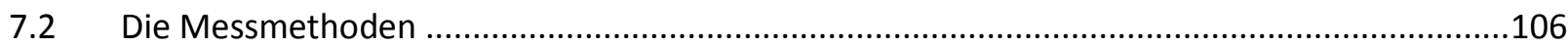

7.3 Die Patientenrekrutierung und der Drop-out........................................................... 107

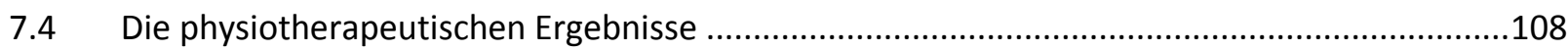

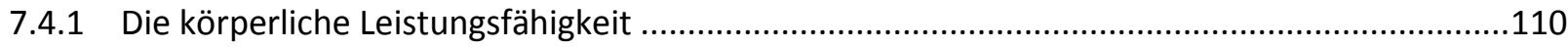

7.5 Die psychoonkologischen Ergebnisse .................................................................

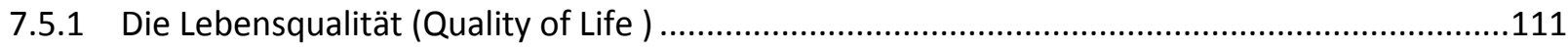

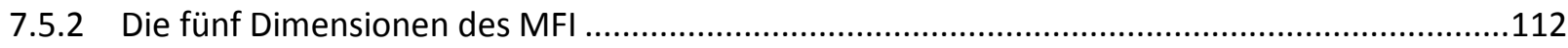

7.5.3 Ängste und Depressionen (HADS) ....................................................................... 114

7.6 Einfluss von psychischem und physischem Stress auf die Immunologie .............................116

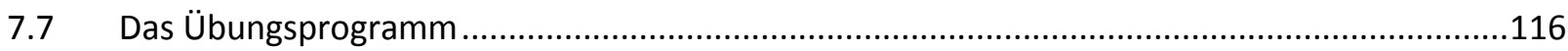

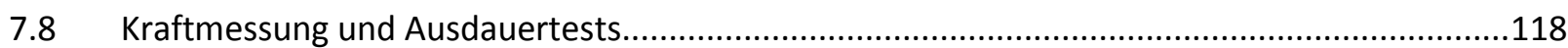




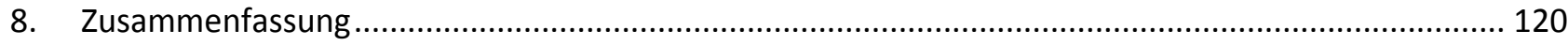

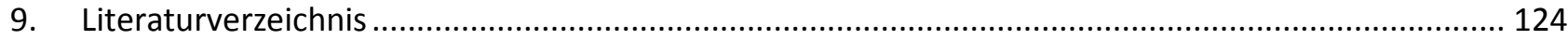

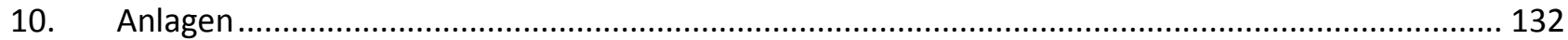




\section{Abbildungsverzeichnis}

Abbildung 1: Teufelskreis "mangelnde Aktivität und Fatigue“

Abbildung 2: Einfluss von Ausdauersport auf psychoneuroimmunologische Parameter ..26

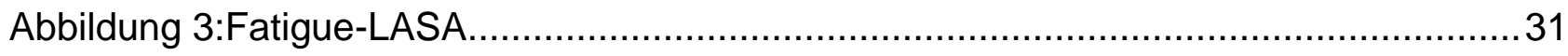

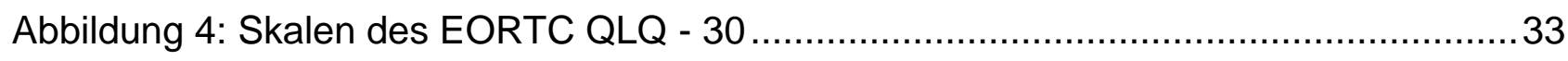

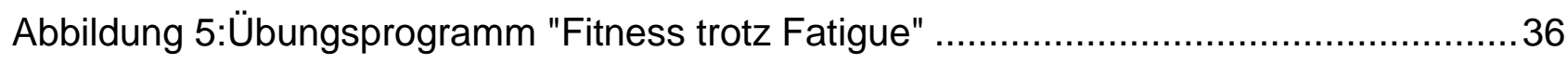

Abbildung 6: Screening und Randomisierung der Patientinnen ........................................ 39

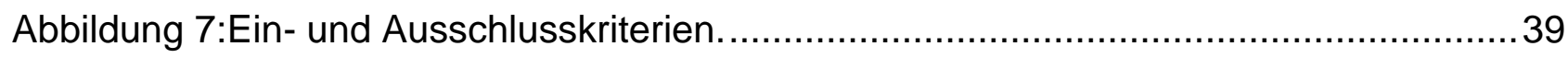

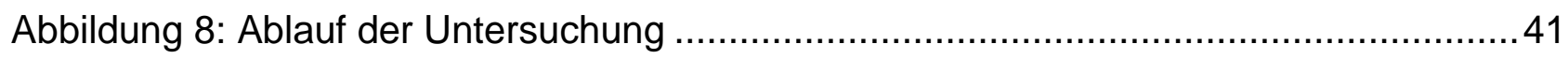

Abbildung 9: Übersicht über die lokale und allgemeine Ausdauer ................................. 42

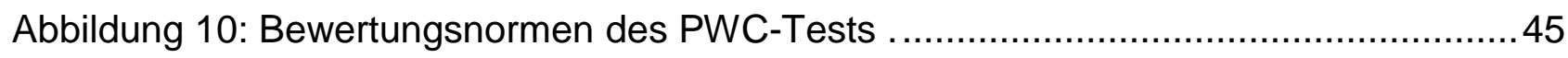

Abbildung 11: Beispiel für die graphische Ermittlung des PWC 150 durch Interpolation bei Über- bzw. Unterschreitung einer Herzschlagfrequenz von 150/min. ............................... 45

Abbildung 12: Grundschema der kybernetischen neuroendokrinen Kontrolle. ..................47

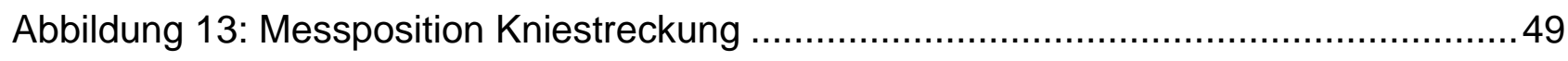

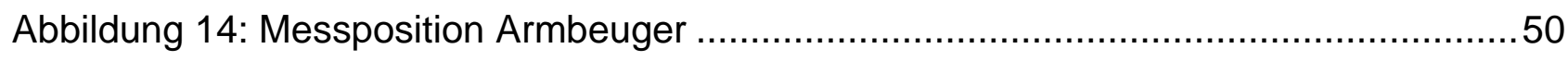

Abbildung 15 a-c: Graphische Darstellung von Alter, Gewicht und Größe der Patientinnen

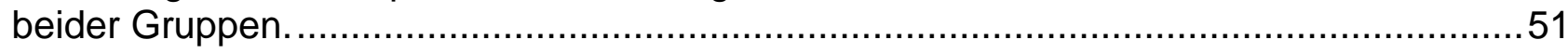

Abbildung 16 a und b: Patientinnenanzahl an den einzelnen Messzeitpunkten beider

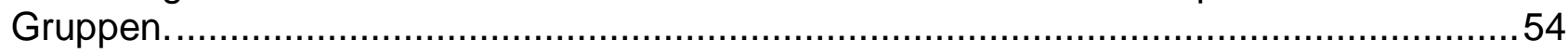

Abbildung 17 a und b: Anzahl der im Monat durchgeführten Kraft- und Dehnübungen

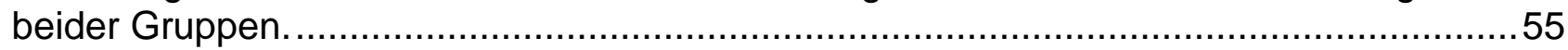

Abbildung 18: Durchschnittlicher Ausdauertrainingsumfang in Std./Monat an den

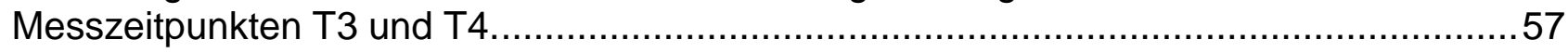

Abbildung 19: Art der durchgeführten Operation bei den Patientinnen der Trainingsgruppe.

Abbildung 20: Art der durchgeführten Operation bei den Patientinnen der

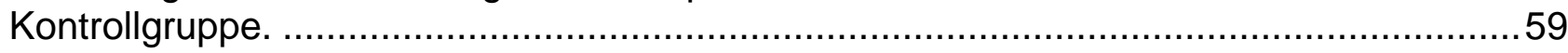

Abbildung 21: Durchführung einer Chemotherapie in der Trainingsgruppe. ......................60

Abbildung 22: Durchführung einer Chemotherapie in der Kontrollgruppe.........................61

Abbildung 23: Durchführung einer Hormontherapie in der Trainingsgruppe.......................62

Abbildung 24: Durchführung einer Hormontherapie in der Kontrollgruppe. ........................63

Abbildung 25: Durchführung einer Strahlentherapie in der Trainingsgruppe. .....................64

Abbildung 26: Durchführung einer Strahlentherapie in der Kontrollgruppe........................65

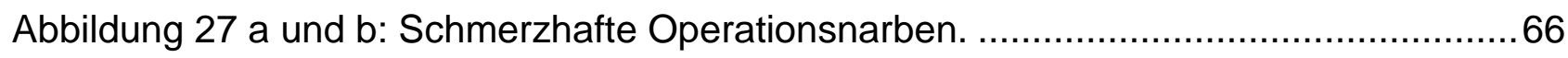

Abbildung 28 a und b: Schwellungen im Operationsbereich........................................66 
Abbildung 29 a und b: Schmerzhafte Verspannungen im Schulterbereich zum Messzeitpunkt T1

Abbildung 30 a und b: Schmerzhafte Verspannungen im Schulterbereich zum Messzeitpunkt T3

Abbildung 31 a und b: Schmerzhafte Verspannungen im Schulterbereich zum Messzeitpunkt T4......

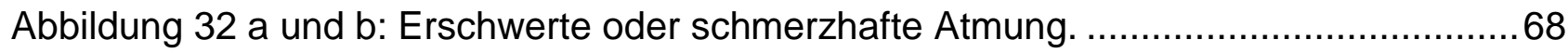

Abbildung 33 a und $b$ : Sensibilitätsstörungen der Hand.

Abbildung 34 a und b: Gefühlter Kraftverlust in Arm oder Schulter zum Messzeitpunkt T1

Abbildung 35 a und b: Gefühlter Kraftverlust in Arm oder Schulter zum Messzeitpunkt T3.

Abbildung 36 a und b: Gefühlter Kraftverlust in Arm oder Schulter zum Messzeitpunkt T4.

Abbildung 37 a und b: Einschränkungen im täglichen Leben zum Messzeitpunkt T1 1 ........71

Abbildung 38 a und b: Einschränkungen im täglichen Leben zum Messzeitpunkt T3.........71

Abbildung 39 a und b: Einschränkungen im täglichen Leben zum Messzeitpunkt T4.........72

Abbildung 40 a und b: Sport vor der Erkrankung. ................................................... 72

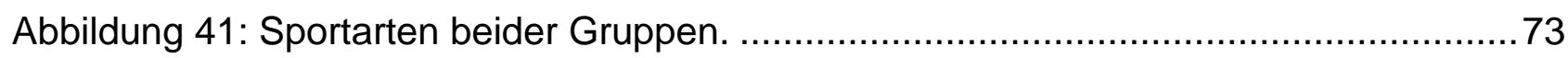

Abbildung 42 a und b: Einschätzung körperlicher Leistungsfähigkeit zum Messzeitpunkt T1

Abbildung 43 a und b: Einschätzung körperlicher Leistungsfähigkeit zum Messzeitpunkt T3.

Abbildung 44 a und b: Einschätzung körperlicher Leistungsfähigkeit zum Messzeitpunkt T4

Abbildung 45: Graph der allgemeinen Fatigue des MFI für die Trainings- und Kontrollgruppe von T1-T4.

Abbildung 46 a und b: Graphen der physischen Fatigue des MFI für die Trainings- und Kontrollgruppe von T1-T4.

Abbildung $47 \mathrm{a}$ und b: Graphen der mentalen Fatigue des MFI für die Trainings- und Kontrollgruppe von T1-T4.

Abbildung 48 a und b: Graphen der reduzierten Aktivität des MFI für die Trainings- und Kontrollgruppe von T1-T4.

Abbildung 49 a und b: Graphen der reduzierten Motivation des MFI für die Trainings- und Kontrollgruppe von T1-T4.

Abbildung 50: Global health status des EORTC der Trainings- und Kontrollgruppe von T1 bis T4.

Abbildung 51 a und b: Physical Functioning des EORTC der Trainings- und Kontrollgruppe von $\mathrm{T} 1$ bis $\mathrm{T} 4$. 
Abbildung 52 a und b: Cognitive Functioning des EORTC der Trainings- und Kontrollgruppe von T1 bis T4.

Abbildung 53: Emotional Functioning des EORTC der Trainings- und Kontrollgruppe von T1 bis T4.

Abbildung 54: Role Functioning des EORTC der Trainings- und Kontrollgruppe von T1 bis T4 .86

Abbildung 55: Social Functioning des EORTC der Trainings- und Kontrollgruppe von T1 bis T4.

Abbildung 56: Graphen der EORTC-Fatigue der Trainings- und Kontrollgruppe von T1 bis T4.

Abbildung 57: Nausea and vomiting des EORTC der Trainings- und Kontrollgruppe von T1 bis T4.

Abbildung 58: Painscore (Schmerzen) des EORTC der Trainings- und Kontrollgruppe von T1 bis T4.

Abbildung 59: Insomnia (Schlaflosigkeit/Schlafstörungen) Score des EORTC der

Trainings- und Kontrollgruppe von T1 bis T4.

Abbildung 60: Dyspnoe des EORTC der Trainings- und Kontrollgruppe von T1 bis T4.....92 Abbildung 61: Appetite loss (Appetitverlust) des EORTC der Trainings- und Kontrollgruppe von T1 bis T4.

Abbildung 62: Constipation (Verstopfungen) des EORTC der Trainings- und Kontrollgruppe von T1 bis T4.

Abbildung 63: Diarrhoe des EORTC der Trainings- und Kontrollgruppe von T1 bis T4....95 Abbildung 64: Financial difficulties (finanzielle Schwierigkeiten) des EORTC der Trainingsund Kontrollgruppe von T1 bis T4.

Abbildung 65 a-d: Angst- und Depressionsscores der Trainings- und Kontrollgruppe von T1 bis T4.

Abbildung 66 a und b: Allgemeine Fatigue bezogen auf den Trainingsumfang. 98

Abbildung 67 a und b: Physische Fatigue bezogen auf den Trainingsumfang. .99

Abbildung 68 a-d: Darstellung der isometrischen Kraftmessung der Beinstrecker und Armbeuger zu Beginn und Ende (T1 und T2) der Rehabilitation in der Trainings- und Kontrollgruppe.

Abbildung 69: Wegstrecke des 6 Min. Gehtests zu Anfang und zum Ende der Rehabilitation in der Trainings- und Kontrollgruppe.

Abbildung 70 a und b: Pulsfrequenzen der Trainingsgruppe an den Messzeitpunkten T1und T2 während unterschiedlicher Wattstufen.

Abbildung 71 a und b: Pulsfrequenzen der Kontrollgruppe an den Messzeitpunkten T1und T2 während unterschiedlicher Wattstufen. 


\section{Tabellenverzeichnis}

Tabelle 1: Mittelwerte und Standardabweichung der Müdigkeitswerte der weiblichen Allgemeinbevölkerung

Tabelle 2 :Beispiel für einen Trainingswochenplan

Tabelle 3: Schnittbestimmung für das Ausdauertraining (Std./Monat)

Tabelle 4: Muskeln und Muskelgruppen

Tabelle 5: Angaben beider Studiengruppen über Alter und Anzahl der Patientinnen in den unterschiedlichen Altersgruppen.

Tabelle 6: Angaben beider Studiengruppen über Größe und Gewicht.

Tabelle 7: Berufliche Situation der Trainingsgruppe zum Messzeitpunkt T1.

Tabelle 8: Berufliche Situation der Kontrollgruppe zum Messzeitpunkt T1.

Tabelle 9: Anzahl der Patientinnen in den einzelnen Scores des Fatigue-LASA.

Tabelle 10: Patientinnenanzahl an den einzelnen Messzeitpunkten beider Gruppen.

Tabelle 11: Prozentuale Teilnehmerinnenanzahl bezogen auf den Messzeitpunkt $\mathrm{T} 1=100 \%$.

Tabelle 12: Anzahl durchgeführter Dehnübungen pro Monat (nach Rehabilitation).

Tabelle 13: Anzahl durchgeführter Kraftübungen pro Monat (nach Rehabilitation)

Tabelle 14: Prozentuale Durchführung der Kraft- und Dehnübungen

Tabelle 15: Unter- über- und durchschnittlicher Trainingsumfang und Anzahl der Patientinnen.

Tabelle 16: Trainingsschnitt in Stunden / Monat zu T3 und T4.

Tabelle 17: Operative Eingriffe bei den Patientinnen der Trainingsgruppe

Tabelle 18: Art der durchgeführten Operation in der Trainingsgruppe.

Tabelle 19: Operative Eingriffe bei den Patientinnen der Kontrollgruppe

Tabelle 20: Art der durchgeführten Operation in der Kontrollgruppe.

Tabelle 21: Durchführung einer Chemotherapie in der Trainingsgruppe.

Tabelle 22: Durchführung einer Chemotherapie in der Kontrollgruppe.

Tabelle 23: Durchführung einer Hormontherapie in der Trainingsgruppe.

Tabelle 24: Durchführung einer Hormontherapie in der Kontrollgruppe.

Tabelle 25: Durchführung einer Strahlentherapie in der Trainingsgruppe.

Tabelle 26: Durchführung einer Strahlentherapie in der Kontrollgruppe.

Tabelle 27: Einschätzung körperlicher Leistungsfähigkeit der Trainingsgruppe zum Messzeitpunkt T1.

Tabelle 28: Einschätzung körperlicher Leistungsfähigkeit der Kontrollgruppe zum Messzeitpunkt T1.

Tabelle 29: Einschätzung körperlicher Leistungsfähigkeit der Trainingsgruppe zum Messzeitpunkt T3. 
Tabelle 30: Einschätzung körperlicher Leistungsfähigkeit der Kontrollgruppe zum Messzeitpunkt T3.

Tabelle 31: Einschätzung körperlicher Leistungsfähigkeit der Trainingsgruppe zum Messzeitpunkt T4.

Tabelle 32: Einschätzung körperlicher Leistungsfähigkeit der Kontrollgruppe zum Messzeitpunkt T

Tabelle 33: Allgemeiner Fatigue-Scores des MFI für die Trainingsgruppe (Tr1-4) und die Kontrollgruppe (K1-4) für die Messzeitpunkte T1-T4.

Tabelle 34: Physischer Fatigue-Score des MFI für die Trainingsgruppe (Tr1-4) und die Kontrollgruppe (K1-4) für die Messzeitpunkte T1-T4.

Tabelle 35: Mentaler Fatigue-Score des MFI für die Trainingsgruppe (Tr1-4) und die Kontrollgruppe (K1-4) für die Messzeitpunkte T1-T4.

Tabelle 36: Reduzierter Aktivität-Score des MFI für die Trainingsgruppe (Tr1-4) und die Kontrollgruppe (K1-4) für die Messzeitpunkte T1-T4.

Tabelle 37: Reduzierter Motivation-Score des MFI für die Trainingsgruppe (Tr1-4) und die Kontrollgruppe (K1-4) für die Messzeitpunkte T1-T4.

Tabelle 38: EORTC global health status der Trainings-und Kontrollgruppe von T1-T4. 82

Tabelle 39: EORTC physical functioning-Score der Trainings- und Kontrollgruppe von T1T4.

Tabelle 40: EORTC cognitive functioning-Score der Trainings- und Kontrollgruppe von T1T4.

Tabelle 41: EORTC emotional functioning-Score der Trainings- und Kontrollgruppe von T1-T4.

Tabelle 42: EORTC role functioning-Score der Trainings- und Kontrollgruppe von T1-T4.86

Tabelle 43: EORTC social functioning-Score der Trainings- und Kontrollgruppe von T1-T4.

Tabelle 44: EORTC Fatigue-Score der Trainings- und Kontrollgruppe von T1-T4.

Tabelle 45: Nausea und vomiting (Erbrechen) der Trainings- und Kontrollgruppe von T1T4.

Tabelle 46: Pain (Schmerzen) der Trainings- und Kontrollgruppe von T1-T4. 90

Tabelle 47: Insomnia (Schlaflosigkeit) der Trainings- und Kontrollgruppe von T1-T4. 91

Tabelle 48: Dyspnoe der Trainings- und Kontrollgruppe von T1-T4.

Tabelle 49: Appetite loss (Appetitverlust) der Trainings- und Kontrollgruppe von T1-T4. 93

Tabelle 50: Constipation (Verstopfungen) der Trainings- und Kontrollgruppe von T1-T4. 94

Tabelle 51: Diarrhoe der Trainings- und Kontrollgruppe von T1-T4.

Tabelle 52: Financial difficulties (finanzielle Schwierigkeiten) der Trainings- und Kontrollgruppe von T1-T4.

Tabelle 53: Angst- und Depressionsscores der Trainings- und Kontrollgruppe von T1 bis $\mathrm{T} 4$ 
Tabelle 54: Durchschnittliche Trainingsumfänge in den Monaten 1-3 (bisT3) und 4-6 (bisT4) nach Rehabilitation und ihr Bezug auf die allgemeine Fatigue.

Tabelle 55: Durchschnittliche Trainingsumfänge in den Monaten 1-3 (bisT3) und 4-6 (bisT4) nach Rehabilitation und ihr Bezug auf die physische Fatigue.

Tabelle 56: Pulsfrequenzen (Mittelwerte) 9der Trainings- und Kontrollgruppe während des PWC-Tests vom Start bis zur Erholung nach 4 Minuten. 


\section{Abkürzungsverzeichnis}

$\begin{array}{ll}\text { Mamma-Ca } & \text { Mammakarzinom } \\ \text { WHO } & \text { World Health Organization } \\ \text { LASA } & \text { Linear-Analog-Self-Assessment-Scale } \\ \text { HADS } & \text { Hospital Anxiety and Depression Scale } \\ \text { MFI } & \text { Multidimensional Fatigue Inventory } \\ \text { EORTC } & \text { European Organisation for Research and Treatment of Cancer } \\ \text { Abb. } & \text { Abbildung } \\ \text { Tab. } & \text { Tabelle } \\ \text { u.a. } & \text { unter anderem } \\ \text { z.B. } & \text { zum Beispiel } \\ \text { vgl. } & \text { vergleiche } \\ \text { o.ä. } & \text { oder ähnliches } \\ \text { Tr } & \text { Trainingsgruppe } \\ \text { K } & \text { Kontrollgruppe } \\ \text { T } & \text { Messzeitpunkte }\end{array}$




\section{Einleitung}

Ein Großteil der an Brustkrebs erkrankten Frauen leidet unter dem sogenannten FatigueSyndrom, auch tumorassoziierte Fatigue genannt. Es handelt sich hierbei um einen akuten oder chronischen, krankhaften Erschöpfungszustand, der die Patientinnen extrem belasten kann. Eine erhebliche Lebensqualitätseinschränkung ist häufig die Folge. Ein weiteres Problem ist, dass normale Erholungsmechanismen wie Schlaf, Ruhepausen o.ä. keine Abhilfe schaffen. Daher sind häufig auftretende Symptome Müdigkeit, Schwäche, Konzentrationsstörungen, geringe körperliche Belastbarkeit, aber auch Ängste und Depressionen. Durch die Multikausalität der Fatigue konnte die Ursache noch nicht vollständig geklärt werden. Es ist jedoch bekannt, dass eine akute Fatigue durch den Tumor, aber auch durch die Tumortherapie ausgelöst werden kann. Zusätzliche Faktoren, die eine Fatigue begünstigen können sind die behandlungsbedingte Anämie und behandlungsbedingte Immunschwächen und dadurch ausgelöste Infekte.

Aufgrund der unterschiedlichen Auslöser wird in eine akute und eine chronische Fatigue unterschieden. Die akute Fatigue tritt vorwiegend während der Therapie oder kurze Zeit später auf. Die chronische Fatigue kann auch Jahre nach der Tumorbehandlung noch bestehen. Durch die noch ungeklärte Ursache der Fatigue gibt es verschiedene Therapieansätze. Diverse Studien konnten einen positiven Einfluss von körperlicher Aktivität auf die Fatigue nachweisen (Cramp \& Daniel, 2008).

Die positiven Einflüsse von körperlicher Aktivität auf den gesamten Organismus sind auch in anderen therapeutischen Bereichen hinreichend erforscht und bekannt. Lange Zeit galt jedoch sportliche Belastung im Zusammenhang mit einer Tumorerkrankung als eher kontraproduktiv. Die Frage war, ob ein bereits geschwächter Körper der Belastung standhalten könne.

Diverse Studien zu diesem Thema konnten bereits nachweisen, dass besonders Ausdauer- und moderates Krafttraining einen positiven Einfluss auf die chronische Fatigue haben (Dimeo, Fetscher, Lange, Mertelsmann, \& Keul, 1997).

Das von der deutschen Fatigue Gesellschaft entwickelte Übungsprogramm „Fitness trotz Fatigue" soll Patienten helfen, durch moderate und selbst bestimmte Belastung, den Stoffwechsel anzuregen und die chronische Fatigue zu verbessern. Ziel der vorliegenden Arbeit war es, die Einsetzbarkeit und Effektivität dieses Übungsprogramms zu testen.

Im anschließenden allgemeinen Teil werden zunächst grundlegende Dinge zur Therapie von Brustkrebserkrankungen besprochen, die tumorassoziierte Fatigue erläutert, sowie die 
Einflüsse von Sport und Rehabilitation im physischen und psychischen Bereich erläutert. Im Kapitel Patientinnen und Methoden werden die einzelnen Arbeitsschritte der vorliegenden Studie erklärt. Danach werden die einzelnen Messinstrumente und Fragebögen in ihren Funktionen erläutert und ihre Einsetzbarkeit erklärt. Es folgt die Darstellung des Übungsprogramms. Die einzelnen Kraft- und Dehnübungen werden bildlich dargestellt und die Durchführungsvarianten des Übungsprogramms in Intensität und Umfang werden erklärt. Das nächste Kapitel beschreibt die einzelnen Hypothesen der Arbeit.

Der folgende Ergebnissteil stellt die Auswertung der Ergebnisse, d.h. der Fragebögen und der Kraft- und Ausdauermessungen graphisch und tabellarisch dar. Es werden die einzelnen Subskalen der Fragebögen und eventuelle Signifikanzen im Zeit-oder Gruppeneffekt gezeigt. Die Diskussion der Ergebnisse schließt sich diesem Kapitel an. Neben den Ergebnissen der vorliegenden Studie werden die Messmethoden und Studiendurchführung diskutiert und mit anderen Studien verglichen.

Abschließend stellt eine Zusammenfassung die Arbeit in einem kurzen Überblick noch einmal dar. Im Anhang finden sich in der Studie benutzte Messinstrumente, Fragebögen, eine genaue Beschreibung der Ergometrie und Anschreiben an die Patientinnen. 


\section{Allgemeiner Teil}

\subsection{Funktionelle Beeinträchtigungen nach Brustkrebstherapie}

Laut World Health Organization (WHO) ist die Definition von Gesundheit das „körperliche, seelische und soziale Wohlbefinden eines Menschen". Durch die Diagnose Krebs und die folgende Behandlung kann es sowohl im physischen als auch im psychischen Bereich zu unterschiedlich stark ausgeprägten Einschnitten kommen. Strahlen- und Chemotherapie wirken sich, besonders durch ihre Nebenwirkungen, stark auf die körperliche Leistungsfähigkeit und das darauf bezogene Wohlbefinden aus. Allein die Diagnose beeinflusst oftmals das seelische Gleichgewicht der Patienten und kann zu starken Ängsten und Depressionen führen. Beide Einschränkungen, also körperliche und seelische, haben häufig einen großen Einfluss auf die sozialen Verhältnisse von Krebspatienten. Zum einen kann die Diagnose, aber auch operative Behandlungen, wie schlimmstenfalls eine Brustamputation, massive Einwirkungen auf das Zusammenleben in einer Partnerschaft haben, oder zu sozialen Isolation führen.

Besonders nach radikalen Brustoperationen leiden die Patientinnen auf physischer Ebene häufig unter Bewegungseinschränkungen, Sensibilitätsstörungen, Fehlhaltungen und Schmerzen durch falsche Belastung und Verspannungen. Da Krebspatienten häufig ein erhöhtes Risiko an Begleiterkrankungen haben, die auch auf physische Inaktivität zurückzuführen sind, sollte hier besonders darauf geachtet werden, ausreichend sportliche Aktivität in den Altag zu integrieren (Mc Tiernan, 2004).

Nach Entnahme der Lymphknoten kann es bei den Patientinnen zur Bildung von Lymphödemen kommen. Diese wirken sich negativ auf die Aktivitäten des täglichen Lebens aus und verhindern ein adäquates Durchführen sportlicher Aktivität.

Die Nebenwirkungen einer Chemotherapie, wie Übelkeit, Erbrechen und ein geschwächtes Immunsystem, beeinflussen ebenfalls die körperliche Leistungsfähigkeit. Die vorher genannten physischen Einschränkungen haben, ebenso wie die Tumorbehandlung mit Chemo- und Strahlentherapie, einen negativen Einfluss auf das psychische Wohlbefinden. Die Krebserkrankung kann schwere Ängste und Depressionen auslösen; die Rate psychischer Erkrankungen ist als hoch einzustufen (Alexander, Palmer, \& Stone, 2010). Neben der Angst, an den Folgen der Erkrankung zu sterben, kommt durch die Operation noch die Angst vor dem Verlust der Weiblichkeit hinzu. 


\subsection{Lebensqualität und Sport}

Häufig kommt es bei den Patientinnen während und nach der Tumorbehandlung zu einer Verschlechterung der aeroben Ausdauerleistungsfähigkeit, einer Verringerung der Muskelkraft und zu einer reduzierten Selbsteinschätzungsfähigkeit. Die Abbildung 1 zeigt den entstehenden Teufelskreis durch Bewegungsmangel.

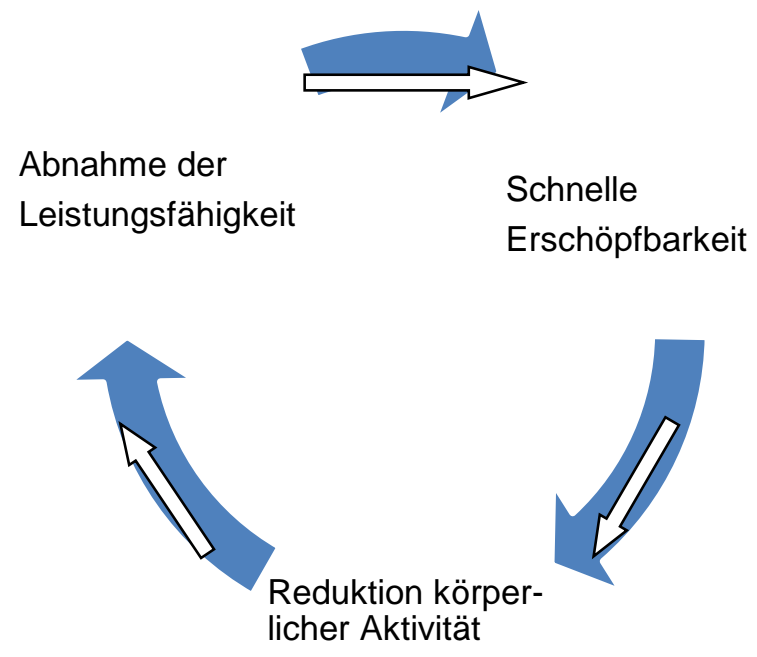

Abbildung 1: Teufelskreis „mangelnde Aktivität und Fatigue“ (Elsner von der Malsburg, 2006)

Dieser Teufelskreis hat einen erheblichen Einfluss auf die Lebensqualität. Die Patientinnen fühlen sich dem Altag nicht mehr gewachsen und kommen schon bei geringer Belastungsintensität sehr schnell an ihre Leistungsgrenze. Valenti et al. zeigten in ihrer Studie an 212 Brustkrebsüberlebenden einen Anstieg der Lebensqualität während eines aktiven Behandlungszeitraums, d.h. wenn die Patientinnen während der Therapie körperlich aktiv waren (Valenti et al., 2008). Headly et al. zeigten in ihrer Studie über ein Sitzendübungsprogramm bei Brustkrebspatientinnen mit fortgeschrittener Erkrankung eine verlangsamte Reduktion des totalen und physischen Wohlbefindens (Headly, Ownby, \& John, 2004).

\subsection{Tumorassoziierte chronische Fatigue}

Chronisch tumorassoziierte Fatigue ist ein eigenständiges Syndrom aus körperlichen, mentalen und emotionalen Komponenten. Eine Definition von David Cella lautet wie folgt:

Die Tumorerschöpfung, auch Fatigue genannt, bedeutet eine außerordentliche Müdigkeit, mangelnde Energiereserven oder ein massiv erhöhtes Ruhebedürfnis, das absolut unverhältnismäßig zur vorangegangenen Aktivitätsänderung ist."(Cella, 1997). 
Die tumorassoziierte Fatigue ist mit 70\% während der Behandlung und 30-40\% nach der Behandlung ein häufig auftretendes Symptom der Tumortherapie (Mock \& Watson, 2004). Auch Jahre nach der Behandlung kann die tumorbedingte Fatigue noch bestehen und somit die berufliche Leistungsfähigkeit einschränken und das Familien- und Sozialleben beeinflussen.

Goedendorp et al. beschrieben in ihrer Arbeit, dass sich während der Behandlung des Tumors die Symptome der Fatigue aufgrund des Tumors selbst und der durchgeführten Behandlungen wie Chemotherapie, Strahlentherapie und Operationene verstärken. Doch auch andere Faktoren wie psychologische Leiden (Depressionen und Ängste), oder die Schlafqualität sollen die Fatigue beeinflussen (Goedendorp, et al., 2008). Die akute Fatigue kann durch den Tumor oder auch durch die Tumortherapie verursacht werden, es ist daher als ein multikausales Geschehen anzusehen. Zusätzliche Begleiterkrankungen wie Infektionen, schwere Anämie, Herzinsuffizienz oder endokrine Störungen können weitere Ursachen einer Fatigue sein.

Aufgrund der unterschiedlichen Auslöser unterscheidet man akute und chronische Fatigue. Tritt die akute Fatigue während der Therapie oder kurze Zeit später auf, so kann die chronische Fatigue noch Monate oder Jahre nach Therapieende bestehen. Als Ursache der akuten Fatigue wird hauptsächlich die Strahlen- und Chemotherapie begleitende Anämie angeführt. Besonders die Bildung des körpereigenen Hormons Erythropoetin, welches zur Bildung der roten Blutkörperchen benötigt wird, kann durch die Strahlen- und Chemotherapie negativ beeinflusst werden. Ebenso eine Schädigung des Knochenmarks und eine damit verbundene Anämie sind möglich. Um die Anämie zu behandeln, werden häufig sogenannte „Erytropoietic agents“ eingesetzt, die die hämatologischen Veränderungen ansprechen und besonders die Lebensqualität der betroffenen Patienten positiv beeinflussen (Straus et al., 2006).

Als nicht pharmakologische Intervention werden Therapien zur Stressbewältigung und Enspannung durchgeführt. Immer mehr Studien weisen auf den positiven Einfluss von körperlicher Aktivität auf die tumorassoziierte Fatigue hin (Cramp \& Daniel, 2008).

\subsubsection{Instrumente zur Erfassung der tumorbedingten Fatigue}

Verschiedene Definitionen der tumorbedingten Fatigue haben die Entwicklung von Messinstrumenten zur Erfassung der tumorbedingten Fatigue beeinflusst. Die meisten Messin- 
strumente beziehen sich auf die "Selbsterfahrung“, das heißt auf subjektive Werte. Unterschieden werden die Messinstrumente in unidimensionale und multidimensionale Tools. Lebensqualitätsinstrumente mit einer Symptomskala für Fatigue, wie die Symptome Distress Scale oder der European Organization for Research and Treatment of cancer (EORTC) QLQ-C30 Quality of life Measure, sollen herausfinden, ob eine tumorbedingte Fatigue vorliegt. Der EORTC QLQ-C30 ist ein Lebensqualitätsfragebogen für Krebspatienten. Er wurde von der European Organisation for Research and Treatment of Cancer über mehrere Jahre entwickelt. Die aktuellste Version 3.0 kommt seit 1997 zur Anwendung.

Bei dem Konzept des Fragebogens wird von einem „integrated modular approach“ gesprochen, d. h. das Kernmodul (C30) kann durch verschiedene tumorspezifische Module ergänzt werden.

Das Ergebnis stellt sich aus mehreren Multiskalen zusammen. Es wird hierbei in eine globale Gesundheitsskala, fünf Funktionsskalen (z. B. Physical Functioning, Emotional Functioning) und neun Symptomskalen (z. B. Fatigue, Pain, Insomnia) unterschieden.

Die multidimensionalen Instrumente wie der Multidimensional Fatigue Inventory (MFI) oder der Functional Assessment of Cancer Therapy-Fatigue Scale (FACT-F) beinhalten unter anderem multidimensionale Symptomskalen (Jean-Pierre et al., 2007).

In der vorliegenden Studie wurde der Multidimensional Fatigue Inventory (MFI) als Fragebogen eingesetzt (Smets et al., 1996). Er ist ein 20 Items umfassendes Instrument zur Selbsteinschätzung und beinhaltet folgende Dimensionen:

- Generelle Fatigue

- Physische Fatigue

- Reduzierte Aktivität

- Reduzierte Motivation

- Mentale Fatigue

Mittels einer Formel wird anschließend ein Fatigue Score berechnet, der sich für Screening- und Verlaufsanalysen eignet.

Die Linear-Analog-Self-Assessment-Scale (LASA) sind analoge, lineare Selbstbeurteilungsmaße. Die Linear-Analog-Skala, die in dieser Studie verwendet wurde, misst die Selbstbeurteilung der Fatigue anhand von aufgetragenen Distanzmaßen. Hier wird von ei- 
nem subjektiven Gefühl der Erschöpfung ausgegangen, so dass die Fatigue qualitativ auf zuverlässige Weise gemessen wurde. An den Endpunkten der Linie stehen jeweils gegensätzliche Aussagen.

Die Fatigue-LASA Skala hat den Vorteil, dass sie leicht verständlich und sehr schnell zu bearbeiten ist.

Eine valide und qualitativ zuverlässige Messung der Fatigue wird durch den Messbogen garantiert.

\subsection{Rehabilitationstraining und medizinische Trainingstherapie}

Die verminderte körperliche Leistungsfähigkeit ist eine der wichtigsten Indikationen für die Anwendung von therapeutischem Training. Besonders die Verbesserung der motorischen Grundeigenschaften Ausdauer und Kraft ist ein erstrangiges Ziel im Bereich der Rehabilitation.

Eine besondere Erwähnung verdient das Krafttraining als therapeutische Maßnahme in der Behandlung und Rehabilitation chronisch kranker und/oder alter Menschen, da Krafttraining bislang in diesem Bereich, hauptsächlich auf Grund traditioneller Vorurteile, praktisch nicht existent war. Dabei ist auch die Kraftleistungsschwäche häufig die eigentliche Ursache für schwerwiegende Funktionsdefizite (Raschka \& Vogel, 2010).

Die Schwächen chronisch kranker Menschen bestehen fast immer aus zwei Komponenten:

- dem Funktionsdefizit, das durch die krankheitsbedingte Schädigung der betroffenen Organe verursacht ist und

- der durch den Bewegungsmangel bedingten „normalen“ Schwäche.

Daraus lassen sich die Trainingsprinzipien der Rehabilitation entwickeln, die im Folgenden näher beschrieben werden. Anfang der 80er Jahre wurden Normwertkategorien von Israel aufgestellt. Diese befassen sich auch heute noch mit den Belastungsparametern in einzelnen Stadien der Gesundheit. Sie sind als eine Abwandlung der eigentlich bei Gesunden geltenden trainingswissenschaftlichen Normen zu verstehen.

Die Minimalnorm beschreibt den präpathologischen Zustand mit dazugehörender Leistungsschwäche, Belastungsintoleranz und, mit geringen Einschränkungen durchführbare 
Alltagstätigkeiten (Ruhe-Herzfrequenz $(\mathrm{HF})=90 \mathrm{~min}^{-1}, V_{\mathrm{O} \text { max }}=30 \mathrm{ml}^{*} \mathrm{~min}^{-1}{ }^{*} \mathrm{~kg}^{-1}$ ). In Klammern gesetzt finden sich die zu dem Normativ gehörenden Ruhewerte.

Die Majoritätsnorm beschreibt, dass alle Alttagstätigkeiten ohne Einschränkungen möglich sind. Man gilt als "gesund“ (Ruhe-HF $=70 \mathrm{~min}^{-1}, \mathrm{~V}_{\mathrm{O} 2 \max }=40 \mathrm{ml}^{\star} \mathrm{min}^{-1 *} \mathrm{~kg}^{-1}$ ). Die Optimalnorm beschreibt die stabile Gesundheit mit vielseitiger Leistungsfähigkeit und Belastbarkeit, sowie erhöhte Beweglichkeit (hier des Hüftgelenks, Beweglichkeitskenngrößen nach Freiwald (Freiwald \& Engelhardt, 1994) ohne Instabilitätszeichen (Ruhe-HF $=55 \mathrm{~min}^{-1}$, $\left.V_{\text {O2max }}=52 \mathrm{ml}^{*} \min ^{-1 *} \mathrm{~kg}^{-1}\right)$. Auf die Beweglichkeitskenngrößen soll hier nicht weiter eingegangen werden. Abschließend beschreibt die Spezialnorm die Leistungsnorm für gesellschaftlich determinierte Tätigkeiten mit hochgradiger Ausprägung der Beweglichkeit in einzelnen oder allen Gelenksystemen (Ruhe-HF $=40 \mathrm{~min}^{-1}, \mathrm{~V}_{\mathrm{O} \max }=75 \mathrm{ml}^{*} \mathrm{~min}^{-1} \mathrm{~kg}^{-1}$ ) (Israel, 1982). Wird in der "gesunden" Gesellschaft versucht, die Kunden oder präventivtrainierenden Patienten von der Majoritätsnorm zur Optimalnorm zu verhelfen, kann bei chronischen Fatiguepatienten nur die Minimalnorm das Ziel sein. Einige erreichen dieses Ziel recht schnell, um sich dann weiterlaufend die Majoritätsnorm als Ziel zu setzten. Grundsätzlich muss vor der Rehabilitation die Normwertkategorie festgelegt werden, um den Patientinnen eine optimale Belastung zukommen zu lassen.

\subsubsection{Trainingsprinzipien in der Rehabilitation}

Das Training in der Rehabilitation ist an individuellen anforderungs-, verletzungs-, bzw. erkrankungsbezogenen Zielen ausgerichtet, unterscheidet sich grundsätzlich aber nicht von den Trainingsprinzipien in anderen Bereichen des Sports. Allerdings ist bei der Trainingsplanung in der Rehabilitation die besondere körperliche, psychische und soziale Situation des Patienten zu berücksichtigen und daher mit großer Umsicht vorzugehen. Entsprechend der Wirkung im Trainingsverlauf werden Prozesse unterschieden, die

- eine Anpassung auslösen,

- Anpassungserscheinungen optimieren und

- eine Anpassung in spezifische Richtungen lenken

(Froböse, Nellessen-Marks \& Wilke, 2010). 
Um diese Anpassungen zu erreichen, werden bestimmte Belastungsnormative als Steuerelemente genutzt:

- Die Trainingsinhalte beschreiben die gewählte Übung mit dem entsprechenden Schwierigkeitsgrad.

- Die Trainingsfrequenz gibt die Häufigkeit des Trainings pro Woche an.

- Der Trainingsumfang beschreibt die Menge des Trainings, z.B. bewegte Gewichte oder gelaufene Kilometer.

- Die Trainingsdichte gibt das Verhältnis von Belastungs- und Pausenzeiten wieder bzw. die Trainingstage im Verhältnis zu den Regenerationstagen.

- Die Trainingsintensität wird meist im Bezug auf die individuelle maximale Leistungsfähigkeit angegeben.

- Die Trainingsdauer gibt die gesamte zeitliche Dauer entweder der Trainingseinheit oder des Trainings in der Woche an.

(Froböse, Nellessen-Marks, \& Wilke, 2010).

Da der tumorassoziierten Fatigue meist mehrere Ursachen zugrunde liegen, muss auch die folgende Therapie und ihre Ziele auf die Fatigue abgestimmt werden. Vgl. Israel, 1982. Adamsen et al. beschrieben in ihrer Arbeit über die Einflüsse von hochintensivem Kraftund Ausdauertraining während der Chemotherapie, dass für einige Patienten die Diagnose und Therapie häufig gleichgesetzt wird mit einem sehr inaktivem Altag, der zu einer Reduzierung der Muskelmasse und Muskelkraft führt (Adamsen, et al., 2009). Daher ist es notwendig, sowohl ein gezieltes Krafttrainingsprogramm, als auch ein aerobes Ausdauertraining in die onkologische Rehabilitation zu integrieren. Das Krafttraining sollte, in Abhängigkeit des Fitnesszustandes des Patienten, in fünf Stufen aufteilen:

1. Aktivierung/Bahnung (intermuskuläre Koordination), Propriozeption

2. Lokales Muskelausdauertraining

3. Vergrößerung des Muskelquerschnitts (Muskelaufbautraining)

4. Steigerung der neuromuskulären Kraftqualitäten

5. Entwicklung vielfältiger und situations(un)abhängiger Kraftqualitäten (Froböse, Nellessen-Marks, \& Wilke, 2010). 
Von den fünf genannten Stufen des Krafttrainings wird der Muskelquerschnittvergrößerung (Stufe 3) die geringste Wichtigkeit in der Therapie der chronischen Fatigue beigemessen. Dies ist auch nicht das Ziel in der Tumor- und Fatiguetherapie, wichtiger sind die Verbesserung der koordinativen Fertigkeiten, der neuromuskulären Kraftqualität und der lokalen Muskelausdauer. Konditionelles Training wird in dem folgenden Punkt näher beschrieben. Weitere Erläuterungen zu den konditionellen Fähigkeiten und den koordinativen Fertigkeiten befinden sich in der Anlage (Anlage 9 und 10).

\subsubsection{Prinzip der Entwicklungs- und Gesundheitsförderung}

Konditionstraining ist so zu gestalten, dass es die physische, psychische bzw. motorische Entwicklung zu keinem Zeitpunkt hemmt und unter verantwortungsbewusster Vermeidung oder weitestgehender Reduzierung von Risiken die Gesundheit allseitig fördert (Grosser, Starischka \& Zimmermann, 2008). Grundsätzlich gilt jedoch, dass der Trainingsreiz eine bestimmte Intensitätsschwelle überschreiten muss, um überhaupt eine Anpassungsreaktion auszulösen und damit trainingswirksam zu sein. Unterschwellige Reize bleiben wirkungslos, überschwellig schwache Reize erhalten das Funktionsniveau, überschwellig starke Reize (optimal) lösen physiologische und anatomische Änderungen aus; zu starke Reize schädigen die Funktionen. Der Schwellenwert des Belastungsreizes hängt von dem Leistungszustand des Sportlers oder des Patienten ab (Grosser, Starischka \& Zimmermann, 2008).

Wird eine Trainingsbelastung über einen längeren Zeitraum nicht variiert, kann dies dazu führen, dass sich der Organismus anpasst und ehemalige, überschwellig wirksame Reize, sogar unterschwellig werden. Die Trainingsbelastung muss nun gesteigert werden. Möglichkeiten der progressiven Belastungssteigerung sind über

- eine Änderung der Belastungskomponenten,

- höhere koordinative Ansprüche,

- eine Erhöhung der Trainingshäufigkeit,

- eine Erhöhung des Trainingsumfangs,

- eine Verkürzung der Pause

- oder eine Erhöhung der Trainingsintensität 
gegeben (Rühl \& Schube, 2003). Allerdings kann es zu jedem Zeitpunkt der Therapie auch zu Störungen oder Komplikationen kommen, so dass die Belastungsfähigkeit entsprechend die Trainingsbelastung angepasst oder eingeschränkt werden muss (Froböse, Nellessen-Marks \& Wilke, 2010).

Um die Anpassungserscheinungen zu optimieren, gelten folgende Prinzipien:

- Das Prinzip der optimalen Gestaltung von Belastung und Erholung.

- Das Prinzip der Wiederholung und Kontinuität.

- Das Prinzip der Periodisierung und Zyklisierung mit einem entsprechenden Phasencharakter im Adaptationsverlauf.

$\mathrm{Da}$ in der Therapie meist veränderte/verlangsamte Regenerationsprozesse zu erwarten sind, ist eine exakte Abstimmung der aufeinander folgenden Reize besonders wichtig (Froböse, Nellessen-Marks \& Wilke, 2010).

\subsubsection{Regeneration}

Die positive und erwünschte Wirkung einer Trainingsanpassung kann nur erfolgen, wenn der Organismus die, dafür entsprechende, zeitliche Gelegenheit erhält, d.h. eine entsprechend lange Trainingspause. Positiv auf die Regeneration kann auch eine ausgewogene, der Belastung angepasste Ernährung wirken.

Unterstützend zur Regeneration bieten sich Massagen, Sauna und Kneipp'sche Bäder (z.B. Wechselbäder) an.

Der Grad der Ermüdung und damit auch die benötigte Erholungs- bzw. Regenerationszeit sind abhängig von der Gesamtbelastung des Trainings, von den Inhalten, den Umfängen und Intensitäten (Froböse, Nellessen-Marks \& Wilke, 2010).

Die speziell in der ambulanten, aber auch in der stationären Rehabilitation zu findenden täglichen Belastungen bedingen, dass eine Variation der Trainingsform (siehe Kapitel 1.4.2) vorgenommen werden muss. Nur dadurch erhält der belastete Organismus ausreichend Zeit, sich an den Trainingsreiz zu adaptieren (Froböse, Nellessen-Marks \& Wilke, 2010). 


\subsection{Psychische und psychophysiologische Einflüsse des Rehabilitations- trainings}

In der Langzeitbehandlung chronisch kranker Patienten spielt die Tatsache, dass sowohl Ausdauer- als auch Krafttraining stimmungsaufhellend und antidepressiv wirken, eine wichtige Rolle.

Diese Veränderungen können auch bei Patienten mit chronischer Fatigue positive Einflüsse auf die Leistungsfähigkeit, die Fatigue und die Lebensqualität haben. Besonderes eine bessere Sauerstoffsättigung im Blut kann eine durch eine Anämie bedingte Fatigue deutlich bessern. Durch die Fatigue schonen sich viele Patientinnen vermehrt, wodurch es zu einer Muskelatrophie kommt. Ist die Atemmuskulatur von der Atrophie betroffen, kommt es zu verminderter kardiopulmonaler Belastbarkeit mit Dyspnoe und eventuellen Tachykardien. Die Sauerstoffversorgung und der Allgemeinzustand der Patientin verschlechtern sich. Die erwähnten positiven Einflüsse durch Training auf den Organismus können auch hier schon durch geringe Belastungsintensitäten präventiv wirken.

Der Abbau von Stresshormonen und die Ausschüttung des Neurotransmitters Serotonin durch körperliche Aktivität, insbesondere Ausdauertraining, können einen positiven Einfluss auf die Lebensqualität haben, da einerseits die Leistungsgrenze positiv verschoben wird und durch den Abbau der Stresshormone und der Ausschüttung des Serotonins Depressionen vermindert und Angstzustände reduziert werden können (Künstlinger, 2002). Des Weiteren kommt es bei bestimmter körperlicher Belastung zu einer vermehrten Ausschüttung körpereigener Opioide, hier besonders dem Endorphin, was beim Menschen ein Wohlgefühl und positive Stimmung hervorruft (Künstlinger, 2002). Bei einer Befragung von 1001 Patientinnen mit Mammakarzinom gaben 90\% an, sich durch die sportliche Betätigung - abseits der spezifischen Therapie - gesünder, aktiver und lebensfroher zu fühlen. (Schünemann, Ascher \& Jänicke, 1993).

\subsubsection{Psychoneuro-Endokrino-Immunologie und Sport}

Unterschiedliche Funktionen des Immunsystems können durch Sport aktiviert werden. Sie betreffen zelluläre Elemente des Immunsystems (Makrophagen, Granulozyten, Natural Killer (NK)-Zellen, aktivierte B- und T-Zellen) wie auch humorale Faktoren (Zytokine, Immunglobuline). Diese immunologische, belastungsbedingte Immunreaktion spielt nicht nur für die Infektabwehr des Körpers eine Rolle, sondern auch für die Regeneration nach sportli- 
cher Belastung. Enge Kopplungen bestehen mit der Psyche und mit dem endokrinologischen System (siehe Abb.2).

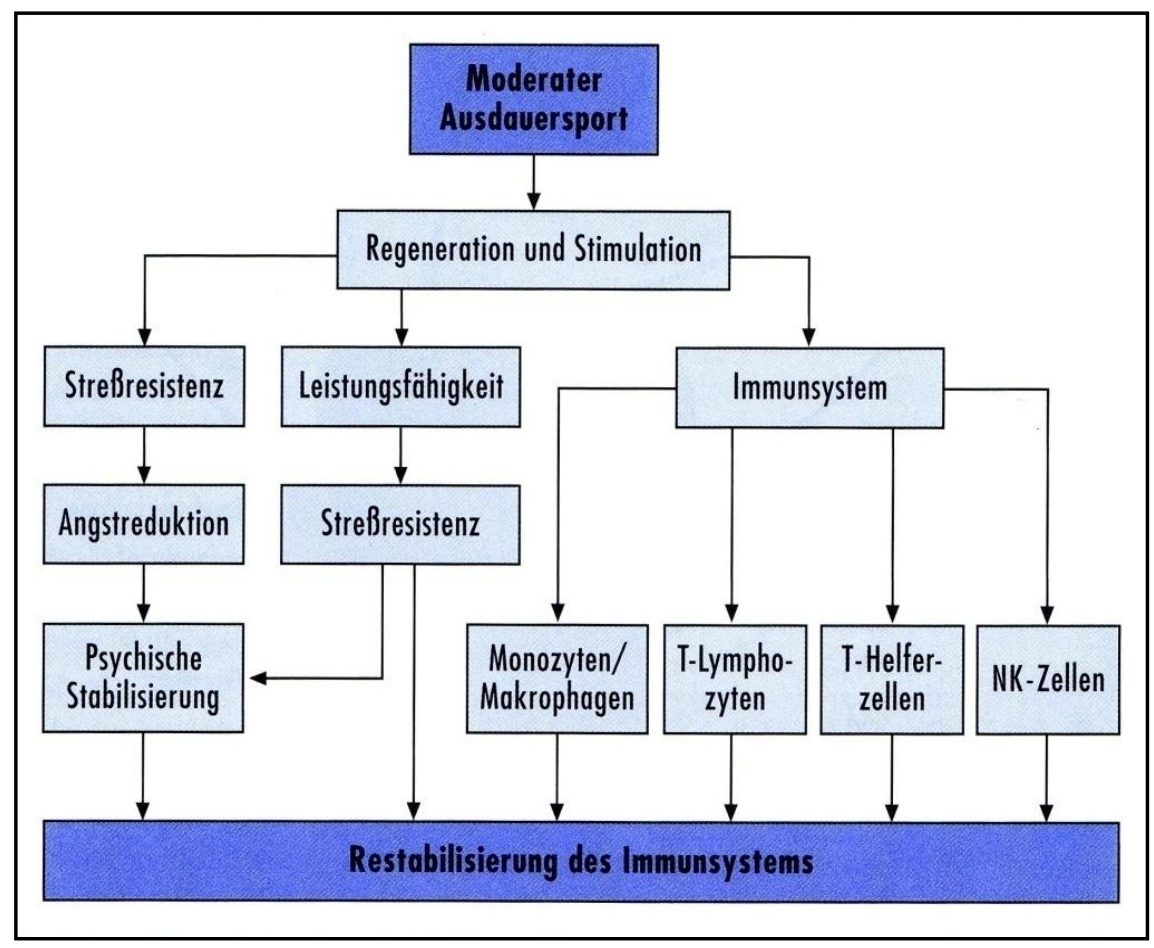

Abbildung 2: Einfluss von Ausdauersport auf psychoneuroimmunologische Parameter (nach Lötzerich u. Uhlenbrock, 1991)

Und selbst die Frage nach der Entstehung und Ausbreitung von Prostatakarzinomen bei Männern und Mammakarzinomen bei Frauen hänget eng mit dem Immunsystem und damit auch mit seiner Beeinflussbarkeit durch sportliche Beanspruchung zusammen (Hollmann \& Strüder, 2009).

\subsection{Sportliche Aktivität während der Tumorbehandlung}

Terson de Palleville et al. zeigten 2007 in ihrer Studie, dass aerobes Training, beginnend eine Woche vor Beginn und fortlaufend während einer Chemotherapie, die Fatigue senken und die Funktionalität der Patientin verbessern kann (Terson de Palleville, Topp \& Swank, 2007).

Auch eine Metaanalyse von 28 Studien über Trainingsprogramme vor und während der Tumorbehandlung von Cramp und Daniel zeigten, dass sportliche Aktivität, ganz gleich ob während oder nach der Tumortherapie, statistisch signifikante, positive Effekte auf die tumorbedingte Fatigue hat. 16 Studien mit 1172 Brustkrebspatientinnen zeigten signifikante positive Effekte. Cramp und Daniel schlussfolgerten aus ihrem Analyseergebnis, dass kör- 
perliches Training während und nach der Tumortherapie als Behandlungsstrategie bei chronischer Fatigue eingesetzt werden sollte (Cramp \& Daniel, 2008).

Auftretende Nebenwirkungen wie Übelkeit, Müdigkeit, Appetitlosigkeit und Gewichtsverlust können durch sportliche Aktivität positiv beeinflusst werden. Mustian et al. konnten in ihrer Studie zeigen, dass beinahe die Hälfte der 749 teilnehmenden Patienten ( $n=356$ bzw. 48\%) während und/oder nach der Tumortherapie Sport betrieben haben. Diese sportliche Aktivität wurde mit weniger schwerwiegenden Nebenwirkungen in Verbindung gebracht (Mustian et al., 2006).

\subsection{Rehabilitation}

Die allgemeine Definition von Rehabilitation lautet:

„Rehabilitation bedeutet das Wiederherstellen des körperlichen, geistigen oder seelischen Zustands nach schwerem Unfall oder schwerer Krankheit zur Wiedereingliederung in Arbeit, Beruf und Gesellschaft“" (Deutsche Rentenversicherung, 2009).

\subsubsection{Rehabilitation nach der Tumortherapie}

In der Onkologie ist die Rehabilitation heutzutage ein fester Bestandteil der Krebsnachsorge. Verschiedenste Therapie- und Bewegungsangebote sollen eine möglichst schnelle Wiederherstellung des allgemeinen Gesundheitszustandes gewährleisten. Häufige Bestandteile der onkologischen Rehabilitation sind Bewegungs- Physio- und Gruppentherapie sowie Klang- und Kunsttherapie. Bartsch et al. schrieben in einem Artikel:

„It is the aim of medical and psychological rehabilitation programs to decrease some of these treatment- and disease-related disabilities and impairments or at least give patient support in the adaption process and in recovering physical and psychological activities during daily life"(Bartsch, Weis \& Moser, 2003).

Da die Diagnose Krebs und deren Therapie alle drei Ebenen (physische, psychische und soziale) der Lebensqualität betreffen wird ein multidisziplinärer und ganzheitlicher Therapieansatz benötigt. Das biopsychosoziale Modell der WHO besagt, dass Gesundheit die ausreichende Kompetenz des Systems „Mensch“ ist, beliebige Störungen auf beliebigen Systemebenen autoregulativ zu bewältigen. Krankheit stellt sich ein, wenn der Organismus die autoregulative Kompetenz zur Bewältigung von auftretenden Störungen auf beliebigen Ebenen des Systems „Mensch“ nicht mehr ausreichend zur Verfügung stellen kann 
und relevante Regelkreise für die Funktionstüchtigkeit des Individuums überfordert sind oder ausfallen (Egger, 2005).

\subsection{Krebsnachsorge und ambulante Krebssportgruppen}

Auf Initiative des Frauenbeirates des Landessportbundes Nordrhein-Westfalen wurden erstmals 1980 ambulante Sportgruppen für Krebsbetroffene eingerichtet, insbesondere für Frauen nach Brustkrebs (Deutscher Sportärztebund e.V., 1996).

Die Gruppentherapie, welche eine gewisse Anzahl von Patientinnen mit ähnlichen Funktionsstörungen voraussetzt, hat insbesondere Vorteile in Bezug auf psychologische Aspekte. Die Gruppentherapie kann die Motivation positiv beeinflussen, zumal die einzelnen $\mathrm{Pa}$ tientinnen mit anderen in einen Erfahrungsaustausch treten und positive Anregungen für sich selbst erhalten; sie erfährt, dass sie nicht allein betroffen ist (Stein \& Greitmann, 2005). 


\section{Hypothesen}

Folgende Hypothesen wurden vor Beginn der Studie aufgestellt:

1. Durch das Sport- und Bewegungsprogramm der Klinik sowie durch die parallel laufenden anderen Therapieformen, wird in beiden Gruppen eine Verbesserung der Kraft- und Konditionswerte festzustellen sein. Konditionelle Verbesserungen und Adaptation der Kraft sind dabei größtenteils auf Verbesserungen der koordinativen Fähigkeiten zurück zu führen.

2. Die Messwerte bzw. die erreichten Scores in den Fragebögen über die gesundheitsbezogenen Lebensqualität und für die einzelnen Parameter der Lebensqualität wie Depressionen, Angst und Fatigue in ihren einzelnen Untergruppen werden bei allen Teilnehmerinnen am Ende der stationären Rehabilitation verbessert sein. Am dritten und vierten Messzeitpunkt werden die Messwerte der Kontrollgruppe unverändert gegenüber dem zweiten Messzeitpunkt oder sogar wieder schlechter sein. In der Trainingsgruppe wird es jedoch zu einer weiteren Verbesserung der Messwerte kommen.

3. Das Übungsprogramm „Fitness trotz Fatigue“ regt zum selbstständigen regelmäßigen Trainieren an, die Therapietreue seitens der Patientinnen kann erzielt werden. Nach Beendigung der Rehabilitationsmaßnahme stellen die Patientinnen ohne Übungsprogramm ihre sportliche Aktivität wieder ein oder lassen eine UnregelmäBigkeit aufkommen.

4. Die durch Operationen eingeschränkte Beweglichkeit sowie die verlorengegangene Kraft und somit auch die subjektive Einschätzung der individuellen Leistungsfähigkeit werden sich in der Trainingsgruppe zum Positiven verbessern. 


\section{Patientinnen und Methoden}

Zur Erhebung von Daten bezogen auf Fatigue, Lebensqualität, Ängste und Depressionen kamen verschiedene standardisierte Fragebögen zum Einsatz. Neben diesen Fragebögen wurden leistungsdiagnostische Verfahren durchgeführt, um die isometrische Maximalkraft und die aerobe Ausdauerleistungsfähigkeit zu bestimmen. Nach dem ersten Testdurchlauf (T1) wurden die Patientinnen der Trainingsgruppe in das Übungsprogramm „Fitness trotz Fatigue" eingewiesen. Sie erhielten zusätzlich eine Broschüre, in der das Übungsprogramm in Bild und Schrift erklärt ist. Die Patientinnen der Kontrollgruppe sollten dem Rehabilitationsprogramm der Klinik weiter folgen.

Die Zeit in der Rehabilitationsklinik sollte von der Trainingsgruppe genutzt werden, um das Übungsprogramm kennen zu lernen und sich mit den Übungen vertraut zu machen. Hier galt, wie auch in den sechs Monaten Trainingsphase nach der Rehabilitation, dass eine $2 x$ wöchentliche Durchführung des Übungsprogramms als 100 Prozent Trainingsumfang im Bezug auf das Übungsprogramm gewertet wurde. Die Patientinnen konnten innerhalb des Übungsprogramms die Intensität selber wählen. Vorgegebene Intensitäten waren $2 \times 7$, 2x15 oder 3×20 Wiederholungen. Da alle Patientinnen einer anderen Normwertkategorie unterlagen, war es nötig, den Patientinnen eine gewisse freie Trainingsumfanggestaltung zu ermöglichen. Das Training mit dem Teraband oder dem eigenen Körpergewicht erlaubte den Teilnehmerinnen zusätzlich noch, den Widerstand zu variieren und den persönlichen Bedürfnissen anzupassen. Somit war für den Kraftübungsteil dafür gesorgt, dass jeder Teilnehmerin ihren individuellen Belastungsumfang erreichen konnte.

Darüber hinaus wurden die Patientinnen angehalten, zusätzlich zum Übungsprogramm $2 x$ wöchentlich eine Ausdauersportart, wie zum Beispiel Nordic Walking, durchzuführen und die Häufigkeit und Dauer der Einheiten ebenfalls zu dokumentieren. 


\section{Fragebögen}

Die folgenden Abschnitte beschreiben die zur Datenerhebung benötigten Fragebögen. Es wird erklärt, um welche Art von Screeninginstrument es sich handelt und in welchem Bereich der Studie das Instrument eingesetzt wurde.

\subsection{Fatigue - LASA (Linear-Analogue-Self-Assessment-Scale)}

Die Fatigue-LASA diente im Rahmen der Studie als Screeninginstrument für die Ausprägung der Fatigue. Sie wurde allen Brustkrebspatientinnen am Tag der Anreise vorgelegt. Patientinnen, die einen Wert von $\geq 4$ auf der Erschöpfungsskala (Siehe Abb. 3) angaben, wurden zu einer Informationsveranstaltung über die Studie eingeladen.

\section{Wie erschöpft fühlen Sie sich?}

Bitte machen Sie ein Kreuz auf der Stelle der Skala, die nach Ihrer eigenen Einschätzung Ihren aktuellen Erschöpfungszustand beschreibt!

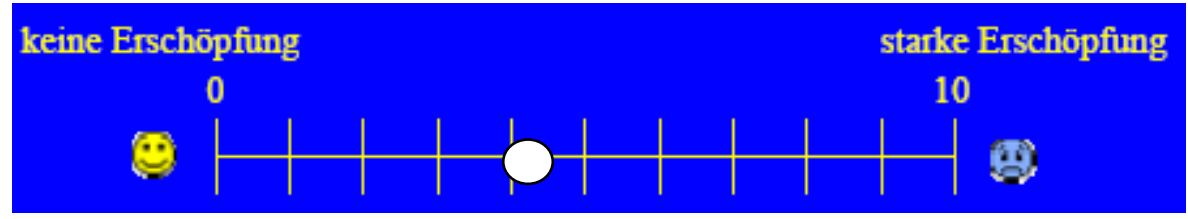

\section{Abbildung 3:Fatigue-LASA}

Der Fatigue-LASA wurde als Screeninginstrument an alle Patientinnen der SonnenbergKlinik verteilt und als Vollerhebung ausgewertet. Die Patientinnen erhielten das Screeninginstrument am Tag der Anreise.

\subsection{HADS (Hospital Anxiety and Depression Scale)}

Der HADS ist ein Fragebogen um rauszufinden, ob bei einem Menschen Ängste und Depressionen vorliegen.

Die HADS enthält Items mit vierstufigen Antwortmöglichkeiten, die zwei Subskalen ergeben (Angstskala und Depressionsskala). Jedes Item des HADS hat eine Likert response Scale. Die Scores dieser Skala basieren auf Summation, wobei hohe Werte größere Belastung bedeuten (Singer et al., 2009). Laut Bjelland et al. liegt der optimale cut-off point bei $\geq 8$ um verdächtige Fälle zu identifizieren und bei $\geq 11$ für sichere Fälle auf beiden Subskalen (Bjelland et al., 2002). Singer et al. gaben in ihrer Arbeit einen Grenzwert von $\geq 13$ 
für Krebspatienten an. Während der Erstbehandlung werden die cut-off points leicht nach oben verschoben. Sie liegen dann bei $\geq 13$ und $\geq 16$ (Singer et al., 2009). Welcher cut-off der richtige für den täglichen Gebrauch ist, wird in der Literatur noch immer kontrovers diskutiert (Singer et al., 2009). Die Skalen des HADS bestehen aus 14 Items, von denen jeweils sieben eine Angst- und eine Depressionssubskala bilden. Die Antworten aus den itembezogenen, vierstufigen Antwortoptionen werden innerhalb der jeweiligen Subskala addiert und kategorisiert. Der HADS kann sowohl als Screeninginstrument als auch zur Verlaufsbeurteilung eingesetzt werden und hat sich innerhalb Europas mittlerweile zum Standardverfahren zur Ermittlung von Angst und Depression entwickelt.

\subsection{MFI (Multidimensional Fatigue Inventory)}

Der MFI setzt sich aus fünf Dimensionen zusammen: Generelle- physische- und mentale Fatigue, sowie reduzierte Aktivität und reduzierte Motivation. Jede der Dimensionen enthält vier Aussagen, für die fünf Antwortkategorien vorgegeben sind. Die Summe der vier zusammengehörigen Aussagewerte ergibt den jeweiligen Skalenwert (Visser \& Smets, 1998).

Die Normwerte für die Müdigkeit in der weiblichen Allgemeinbevölkerung berechneten Schwarz et al. und können der folgenden Tabelle entnommen werden:

\begin{tabular}{|l|l|l|l|}
\hline Frauen & $\mathbf{3 3 9}$ Jahre & $\mathbf{4 0 - 5 9}$ Jahre & $\mathbf{2 6 0}$ \\
\hline Generelle Fatigue & $7.7 \pm 3.2$ & $8.7 \pm 3.5$ & $10.8 \pm 3.7$ \\
\hline Physische Fatigue & $6.8 \pm 3.0$ & $8.2 \pm 3.8$ & $11.1 \pm 4.2$ \\
\hline Reduzierter Akt. & $7.1 \pm 3.0$ & $8.2 \pm 3.7$ & $10.5 \pm 4.0$ \\
\hline Reduziertes Motiv. & $6.7 \pm 2.7$ & $8.0 \pm 3.2$ & $9.9 \pm 3.5$ \\
\hline Mentale Fatigue & $7.1 \pm 3.1$ & $7.8 \pm 3.1$ & $9.2 \pm 3.4$ \\
\hline
\end{tabular}

Tabelle 1: Mittelwerte und Standardabweichung der Müdigkeitswerte der weiblichen Allgemeinbevölkerung (Schwarz, Krauss, \& Hinz, 2003).

\subsection{EORTC QLQ-C 30 Version 3.0}


Der European Organisation for Research and Treatment of Cancer (EORTC) Quality of life Fragebogen ist ein Instrument zur Ermittlung der gesundheitsbezogenen Lebensqualität bei Krebspatienten. Der Kernfragebogen, der QLQ-C30, ist ein Resultat aus über einer Dekade an wissenschaftlichen Untersuchungen (Aaronson et al., 1993). Abbildung 4 zeigt die verschiedenen Funktions-, und Symptomskalen, sowie den Global health Status.

\begin{tabular}{|c|c|c|c|c|c|}
\hline & Scale & $\begin{array}{l}\text { Number } \\
\text { of items }\end{array}$ & $\begin{array}{l}\text { Item } \\
\text { range* }\end{array}$ & $\begin{array}{l}\text { Version } 3.0 \\
\text { Item numbers }\end{array}$ & $\begin{array}{c}\text { Function } \\
\text { scales }\end{array}$ \\
\hline $\begin{array}{l}\text { Global health status / QoL } \\
\text { Global health status/QoL (revised) }{ }^{\dagger}\end{array}$ & QL2 & 2 & 6 & 29,30 & \\
\hline $\begin{array}{l}\text { Functional scales } \\
\text { Physical functioning (revised) } \\
\text { Role functioning (revised })^{\dagger} \\
\text { Emotional functioning } \\
\text { Cognitive functioning } \\
\text { Social functioning }\end{array}$ & $\begin{array}{l}\text { PF2 } \\
\text { RF2 } \\
\text { EF } \\
\text { CF } \\
\text { SF }\end{array}$ & $\begin{array}{l}5 \\
2 \\
4 \\
2 \\
2\end{array}$ & $\begin{array}{l}3 \\
3 \\
3 \\
3 \\
3\end{array}$ & $\begin{array}{l}1 \text { to } 5 \\
6,7 \\
21 \text { to } 24 \\
20,25 \\
26,27\end{array}$ & $\begin{array}{l}\text { F } \\
F \\
F \\
F \\
F\end{array}$ \\
\hline $\begin{array}{l}\text { Symptom scales / items } \\
\text { Fatigue } \\
\text { Nausea and vomiting } \\
\text { Pain } \\
\text { Dyspnoea } \\
\text { Insomnia } \\
\text { Appetite loss } \\
\text { Constipation } \\
\text { Diarrhoea } \\
\text { Financial difficulties }\end{array}$ & $\begin{array}{l}\text { FA } \\
\text { NV } \\
\text { PA } \\
\text { DY } \\
\text { SL } \\
\text { AP } \\
\text { CO } \\
\text { DI } \\
\text { FI }\end{array}$ & $\begin{array}{l}3 \\
2 \\
2 \\
1 \\
1 \\
1 \\
1 \\
1 \\
1\end{array}$ & $\begin{array}{l}3 \\
3 \\
3 \\
3 \\
3 \\
3 \\
3 \\
3 \\
3\end{array}$ & $\begin{array}{c}10,12,18 \\
14,15 \\
9,19 \\
8 \\
11 \\
13 \\
16 \\
17 \\
28\end{array}$ & \\
\hline
\end{tabular}

Abbildung 4: Skalen des EORTC QLQ - 30

\subsection{Dokumentationsbogen}

Der Dokumentationsbogen diente der Ermittlung der soziodemographischen Daten (Alter, Geschlecht, Familienstand etc.). Des Weiteren wurden die Behandlungsarten vor, während und nach der Rehabilitation und weitere Informationen zur Brusterkrankung abgefragt. Ein physiotherapeutischer Fragenteil diente zur Ermittlung von Bewegungseinschränkungen, Sensibilitätsstörungen, subjektivem Kraftverlust und Schwellungen im operierten Gebiet. Abschließend wurden Fragen zu sportlichen Aktivitäten der Patientinnen gestellt (Anlage 6). 


\section{Das Übungsprogramm „Fitness trotz Fatigue“}

Entwickelt wurde das Übungsprogramm „Fitness trotz Fatigue“ durch die „Deutsche Fatigue Gesellschaft“ (2005) mit Unterstützung der Deutschen Sporthochschule in Köln. Prinzip des Übungsprogramms ist es, durch eine Verbesserung der Kraft und Ausdauer, die körperliche Leistungsfähigkeit der Fatiguepatientinnen zu erhöhen. Zur Veränderung der körperlichen Leistungsfähigkeit werden die fünf motorischen Hauptbeanspruchungsformen nach Hollmann und Hettinger genutzt. Diese sind Kraft, Ausdauer, Schnelligkeit, Flexibilität und Koordination (Hollmann \& Hettinger, 2000).

Das Übungsprogramm setzt sich insbesondere mit den Punkten Kraft, Ausdauer und Flexibilität auseinander. Das Übungsprogramm beinhaltet neun Kräftigungsübungen und neun dazugehörige Dehnübungen. Die Übungen sind in Bildern dargestellt und zusätzlich schriftlich erläutert, um eine selbstständige Durchführung so fehlerfrei wie möglich zu gewährleisten.

\section{Kraftübungen}

„Sit-Up's in Rückenlage“

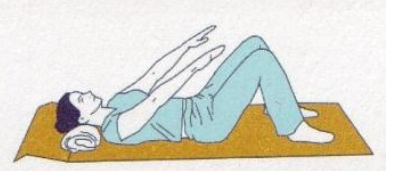

„Schräge Sit-Up's in Rückenlage“

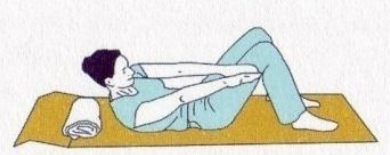

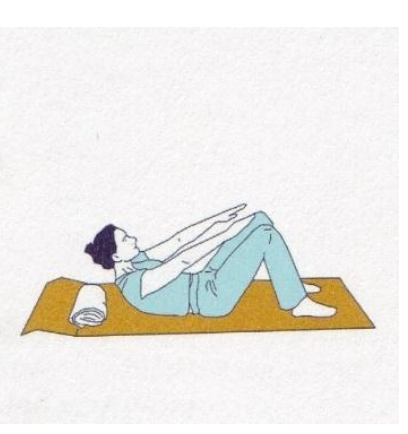

\section{Dehnungsübungen}

„Gestreckte Lanze“

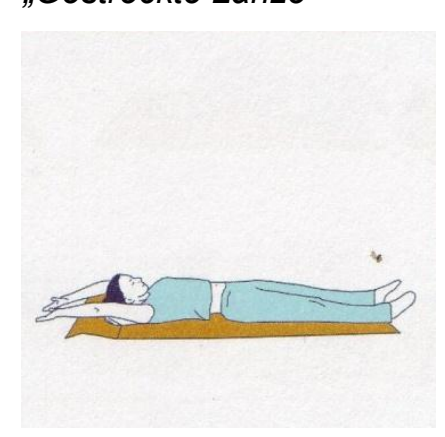

„Scheibenwischer"

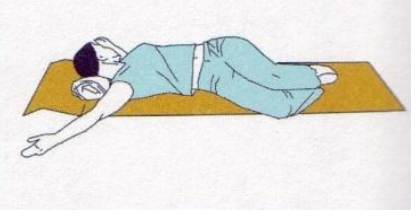


Kraftübungen

„Bridging in Rückenlage“

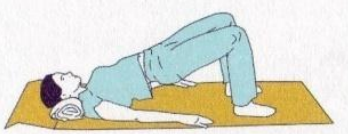

„Schere“

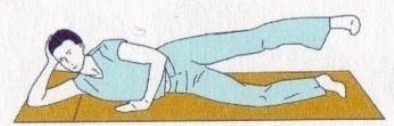

"Schraubstock"

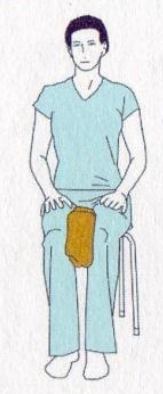

„Stepper"

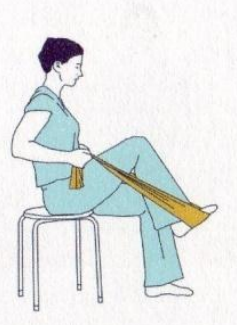

\section{Dehnungsübungen}

„Päckchen“

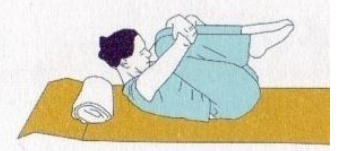

"Triangel"

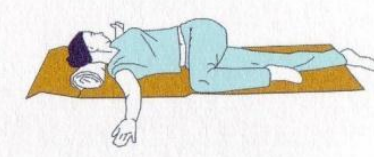

„Pforte“

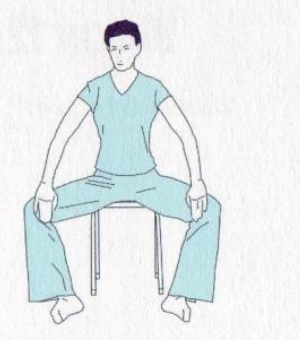

„Seepferdchen“
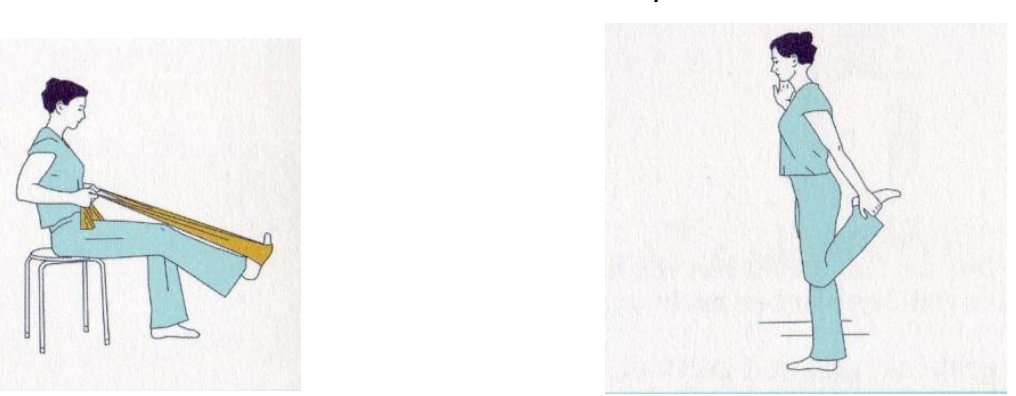


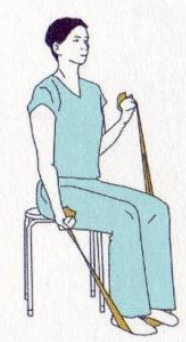

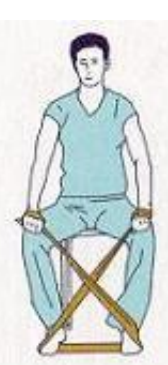

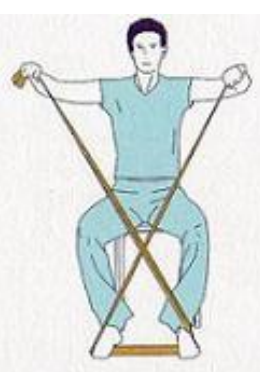

„Rudern“

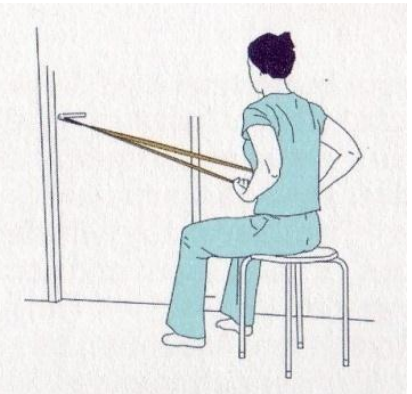

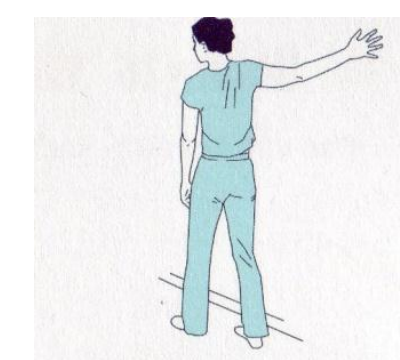

"Winkelhaken

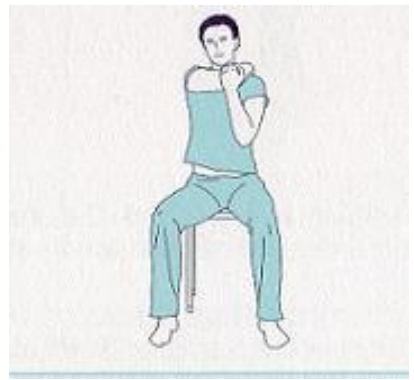

„Hängematte“

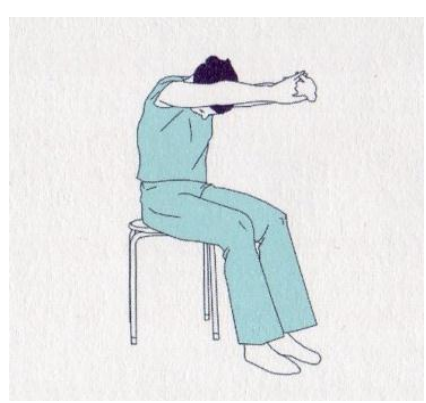

Abbildung 5:Übungsprogramm "Fitness trotz Fatigue" (Deutsche Fatigue Gesellschaft, 2005)

Die Übungsdurchführung wurde von den Patientinnen auf einem Dokumentationsbogen festgehalten werden. Die Patientinnen können die Belastungshäufigkeit selber bestimmen. Das bedeutet, sie legen die Anzahl der Einzelreize pro Serie oder pro Therapieeinheit selber fest (Radlinger, 1998). Hierbei bestehen die Möglichkeiten, 2x7, 2x15 oder 3×20 Wiederholungen pro Übungssatz durchzuführen. Den Belastungsumfang legen die Patientinnen ebenfalls selbst fest, indem sie entscheiden, ob alle Übungen an einem Tag durchgeführt werden, oder ob sie die Übungen auf mehrere Tage verteilen. Das heißt, der Belas- 
tungsumfang beschreibt die Summe aller Belastungsreize pro Therapieeinheit (Radlinger, 1998). Das Übungsprogramm mit seinen Kräftigungsübungen wurde gewählt, um besonders die lokale Ausdauerleistungsfähigkeit zu verbessern.

Ein weiterer Bestandteil des Übungsprogramms ist ein aerobes Ausdauertraining in Form von Nordic Walking. Diese Form des Ausdauertrainings stellt besonders für Sportanfänger und übergewichtige Menschen eine optimale Trainingsmöglichkeit dar. Gefördert werden sollte hier besonders die lokale dynamisch aerobe Ausdauerleistungsfähigkeit. Diese Form der Muskelbeanspruchung liegt dann vor, wenn eine dynamische Arbeit mit kleinen bis mittelgroßen Muskelgruppen (z.B. eines Arms oder eines Beins) durchgeführt wird. Die lokale dynamische aerobe Muskelausdauer ist für die Präventivmedizin und der Rehabilitation sowie für den Leistungssport gleichermaßen von sehr großer Bedeutung (Hollmann \& Strüder, 2009). Die physiologischen Grundlagen zur Verbesserung beruhen auf der Vergrößerung des intrazellulären $\mathrm{O}_{2}$ - Angebots, Verbesserung der Enzymaktivität des aerobes Stoffwechsels, Vermehrung des Myoglobins, und Vergrößerung des lokalen Kohlenhydratdepots (Hollmann et al., 2000). Die Belastungen auf das Skelettsystem sind sehr gering und die Trainingsintensität kann optimal auf die Bedürfnisse des Trainierenden angepasst werden. Das Übungsprogramm „Fitness trotz Fatigue“ bietet als Walkingmethoden die Dauer- oder Tempowechselmethode an. Die Dauermethode besteht aus kontinuierlichem Walken, die Tempowechselmethode beinhaltet einen steten Wechsel des Tempos, zum Beispiel eine Minute schnelles Gehen (Belastungsphase) und eine Minute langsames Gehen (Erholungsphase). Die Intervalle können nach Belastungsvermögen vom Trainierenden verändert werden.

Die grundsätzliche Empfehlung an die Patientinnen dieser Studie war es, die Kraft- und Dehnübungen im täglichen Wechsel mit dem Ausdauerprogramm durchzuführen. Nach zwei Tagen aufeinanderfolgender Belastung sollte ein Tag Pause erfolgen. Allerdings kann eine hohe Leistungsfähigkeit nur dann erreicht werden, wenn Training und Erholung, d.h. Belastung und Wiederherstellung, Schlafen und Wachen, Ruhe und Bewegung zeitlich weitestgehend mit den natürlichen Rhythmen des Organismus übereinstimmen (Oschütz, 1998). Anhand von Messungen gibt Oschütz an, dass die Zeiträume zwischen 10 und 12 Uhr und 16 und 18 Uhr für körperliches Training zu bevorzugen sind, da die Chronobiologie der Trainingszeitpunkte einen Einfluss auf die Effektivität und Qualität des Trainings hat und das Adaptationsniveau der körperlichen Leistungsfähigkeit mitbestimmt (Oschütz, 1998). In der Auswahl der Ausdauersportart hatten die Teilnehmerinnen freie Auswahl. Überwiegend wurde aber Nordic Walking, Fahrradfahren oder Schwimmen als 
Ausdauersportart gewählt. Das bedeutet, dass die Patientinnen den Ausdauerteil selber bestimmen konnten. Das Hauptaugenmerk dieser Studie lag auf dem Kräftigungs- und Dehnungsteil.

\begin{tabular}{|l|c|c|c|l|c|}
\hline \multicolumn{1}{|c|}{ Montag } & Dienstag & Mittwoch & Donnerstag & Freitag & Wochenende \\
\hline Kraft- u. & Ausdauer & Pause & Ausdauer & Kraft- u. & Pause \\
Dehnungs- & & & & $\begin{array}{l}\text { Dehnungs- } \\
\text { übungen }\end{array}$ & \\
übungen & & & & \\
\hline
\end{tabular}

Tabelle 2 :Beispiel für einen Trainingswochenplan

Ziel der onkologischen Rehabilitation ist es, die Belastbarkeit für die Patienten für den Alltag wiederherzustellen. Hierbei spielt die Wiedereingliederung in den Arbeitsprozess eine genauso wichtige Rolle, wie der Erhalt oder die Wiedergewinnung der sozialen Integration. Da die onkologischen Patienten oft physisch und psychisch geschwächt sind und der Großteil sich in der fünften oder sechsten Lebensdekade befindet ist es wichtig, das Training auf die körperlichen Grundvoraussetzungen der Patienten anzupassen.

\begin{tabular}{|l|}
\hline Ausdauertrainingsumfänge \\
\hline Unterdurchschnitt $\leq 4$ Std/Mon. \\
\hline Durchschnitt 5-8 Std./Mon. \\
\hline Überdurchschnitt >8 Std./Mon. \\
\hline
\end{tabular}

Tabelle 3: Schnittbestimmung für das Ausdauertraining (Std./Monat)

Wie in Tabelle 3 dargestellt, wurde die Bewertung des unterdurchschnittlichen Trainingsumfangs bei $\leq 4 \mathrm{Std}$./Mon. angesetzt. Dies würde einem Ausdauertraining von bis zu einer Stunde pro Woche entsprechen. Ein durchschnittlicher Ausdauertrainingsumfang lag zwischen 5 und 8 Stunden/Monat oder 1,25 bis zwei Stunden pro Woche. Als überdurchschnittlichen Trainingsumfang wurde alles über acht Stunden pro Monat oder zwei Stunden pro Woche gewertet.

\subsection{Patientenrekrutierung}

Es wurden ausschließlich Brustkrebspatientinnen rekrutiert, die an der stationären onkologischen Rehabilitationsmaßnahme in der Sonnenbergklinik Bad Sooden-Allendorf teilgenommen haben. Nach einem intensiven Aufklärungsgespräch wurden die Patientinnen, 
die sich zur Teilnahme an der Studie entschlossen hatten randomisiert und in eine Kontrollgruppe und eine Interventionsgruppe eingeteilt. Die Randomisierung nach gerader bzw. ungerader Kalenderwoche stellte sich als problematisch dar, weil die Patientinnen sich innerhalb der Rehabilitation über die Studie unterhielten und häufiger die Frage aufkam, warum einige Teilnehmerinnen ein zusätzliches Übungsprogramm haben und andere nicht. Daher wurde die Randomisierung auf einen 6 Wochenrythmus verändert. Die Begründung, dass in einem 6-wöchigen Rhythmus die Patientinnen ein und derselben Gruppe leichter zu betreuen sind, wurde von den Patientinnen akzeptieren.

\section{Patientinnen mit Brustkrebs in stationärer Rehabilitation}

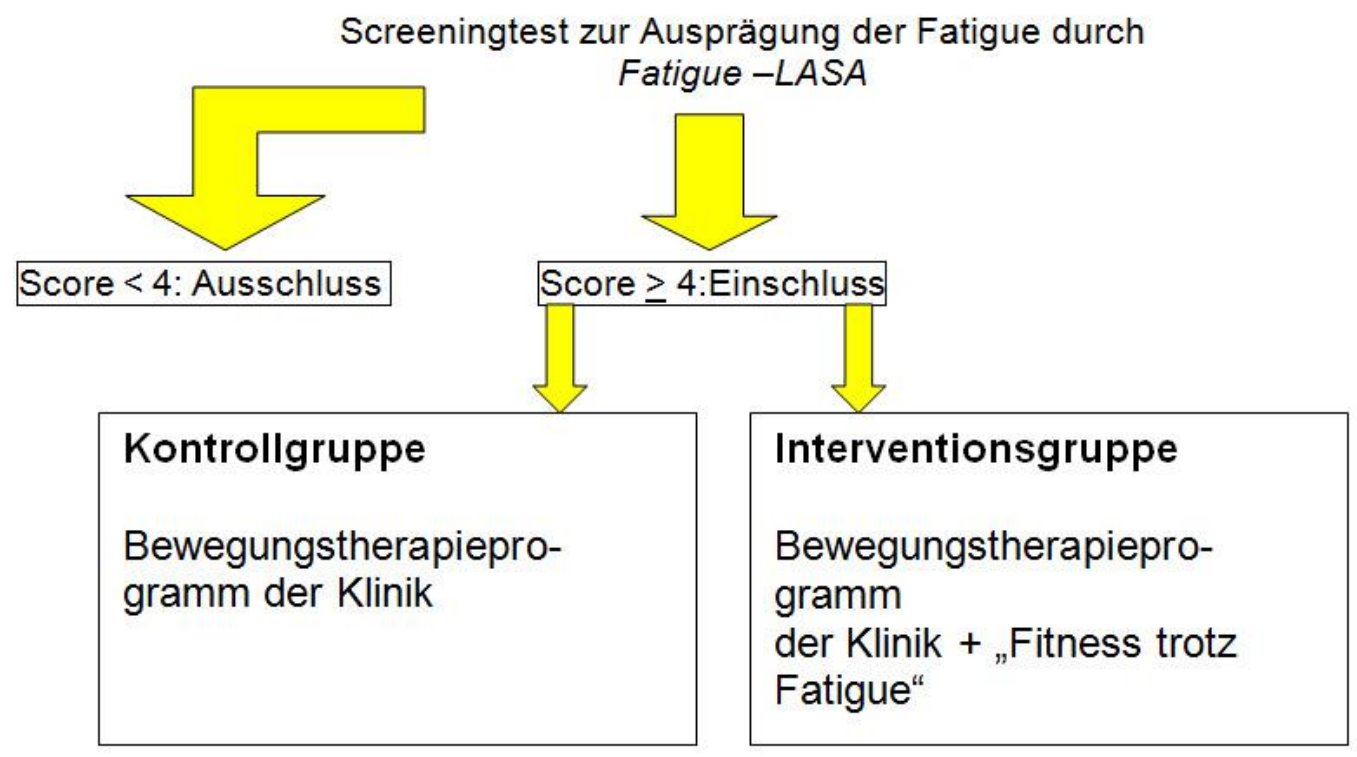

Abbildung 6: Screening und Randomisierung der Patientinnen (Elsner von der Malsburg, 2006)

\begin{tabular}{|l|l|}
\hline Einschlusskriterien & Ausschlusskriterien \\
\hline Erkrankung an Brustkrebs & Fehlende oder zurückgenommene \\
Weibliches Geschlecht, jeder Altersklasse & Einwilligungserklärung \\
PostOP mindesten 6 Wochen & Laufende Chemo,- oder Strahlentherapie \\
Einen Fatigue-Score von mind.4 auf der & Psychiatrische Erkrankungen, außer thera- \\
LASA & pie- \\
Rehabilitationsaufenthalt & begleitende Depressionen \\
& Schwierige Begleiterkrankungen und hoch- \\
& gradige Funktionsstörungen \\
\hline
\end{tabular}

Abbildung 7: Ein- und Ausschlusskriterien. 
Die studienteilnehmenden Patientinnen wurden instruiert, in den drei bis vier Wochen der stationären Rehabilitation das Übungsprogramm so regelmäßig wie möglich durchzuführen. Da die zeitliche und auch körperliche Belastung während der Rehabilitation relativ hoch ist und viele aktive Therapieangebote der Klinik durchgeführt werden, sollte das Übungsprogramm von den Patientinnen an den Therapiealltag angepasst werden.

Unmittelbar nach der Rehabilitationsmaßnahme sollte das Übungsprogramm dann regelmäßig am Wohnort bis sechs Monate nach Rehabilitation durchgeführt werden.

Den Patientinnen der Kontrollgruppe war es nicht untersagt, sportlichen Aktivitäten nachzugehen. Dies sollten sie dann auf einem, ihnen zu den Messzeitpunkten T3 und T4, zugeschickten Fragebogen angeben. Da Patientinnen der Kontrollgruppe gegebenenfalls wieder zu ihren vor der Erkrankung ausgeführten Sportarten zurückkehrten, musste dies mit in die Erhebung aufgenommen werden, um eventuelle Fehlerquellen zu minimieren oder erklären zu können.

\subsection{Studienablauf}

Am Tag der Anreise erhielten die Patientinnen im Zusammenhang mit den Voruntersuchungen den Fatigue-LASA Bogen als Screeninginstrument ihrer Fatigue. Kennzeichneten sie eine Score von $\geq 4$, wurden sie zur Informationsveranstaltung über die Studie eingeladen. In dieser Veranstaltung erfolgte eine Aufklärung über die Studie, damit verbundene Risikofaktoren sowie Informationen zu sportlicher Aktivität bei Brustkrebs und Fatigue.

Mit dem Unterschreiben einer Einwilligungserklärung und eines Aufklärungsschreibens willigten die Patientinnen in die Studienteilnahme ein.

Die Studienteilnehmerinnen wurden zu Beginn ihrer Rehabilitationsmaßnahme (T1) einer Leistungsdiagnostik in Form des PWC- Fahrradergometertests und einem 6 Min. Gehtest unterzogen. Der PWC-Test wurde je nach Alter der Patientinnen bis zu einem Puls von 110 oder 130 Schläge/Minute durchgeführt. Die isometrische Maximalkraft wurde mittels einer Kraftmessdose für die Oberarmbeuger (M. Biceps brachii) und für die Knieextensoren (M. Quadrizeps femoris) ermittelt. Zusätzlich erhielten die Teilnehmerinnen noch einen Fragebogenkatalog. Dieser beinhaltete den MFI (Multidimensional Fatigue Inventory), EORTC QLQ-C30 (Lebensqualitätsfragebogen), HADS (Hospital Anxiety and Depression Scale) und einen soziodemographischen Fragebogen. Neben Daten zur Person enthielt der soziodemographische Fragebogen auch Fragen zur allgemeinen sportlichen Aktivität, Angaben zur Erkrankung und zur gesundheitlichen Situation. Ein physiotherapeutischer 
Teil gab dazu noch Aufschluss über Sensibilitätsstörungen, Kraftverlust und Bewegungseinschränkung im Bereich der operierten Brust und Schulter. Die Teilnehmerinnen wurden in sechswöchigen Blöcken randomisiert und der Interventions- oder Kontrollgruppe zugeteilt. Die Teilnehmerinnen der Kontrollgruppe durchliefen das Rehabilitationsprogramm der Klinik und wurden am Ende (T2) erneut der Leistungsdiagnostik und den Krafttests unterzogen. Dazu erhielten sie erneut den Fragebogenkatalog. Nach Aufnahme der Anschrift kehrten die Teilnehmerinnen an ihren Wohnort zurück und bekamen den Fragebogenkatalog nach drei Monaten (T3) und nach sechs Monaten (T4) zugeschickt.

Die Teilnehmerinnen der Interventionsgruppe durchliefen die gleichen Testreihen zu denselben Zeitpunkten wie die Kontrollgruppe. Zusätzlich bekamen sie zum Messzeitpunkt T1 noch eine Einweisung in das Übungsprogramm „Fitness trotz Fatigue" und das zur Durchführung nötige Equipment. Das Equipment bestand aus einem Teraband, der Anleitung des Übungsprogramms, sowie Fitnessbögen, in denen die Patientinnen den Trainingsumfang und die Intensität dokumentieren sollten. Genau wie den Teilnehmerinnen der Kontrollgruppe, bekamen die Teilnehmerinnen der Interventionsgruppe nach drei Monaten (T3) und nach sechs Monaten (T4) die Fragebögen und zusätzlich neue Fitnessbögen postalisch zugeschickt. Alle Teilnehmerinnen waren angehalten, ihre kompletten Unterlagen nach dem letzten Messzeitpunkt T4 an das Institut zurückzuschicken.

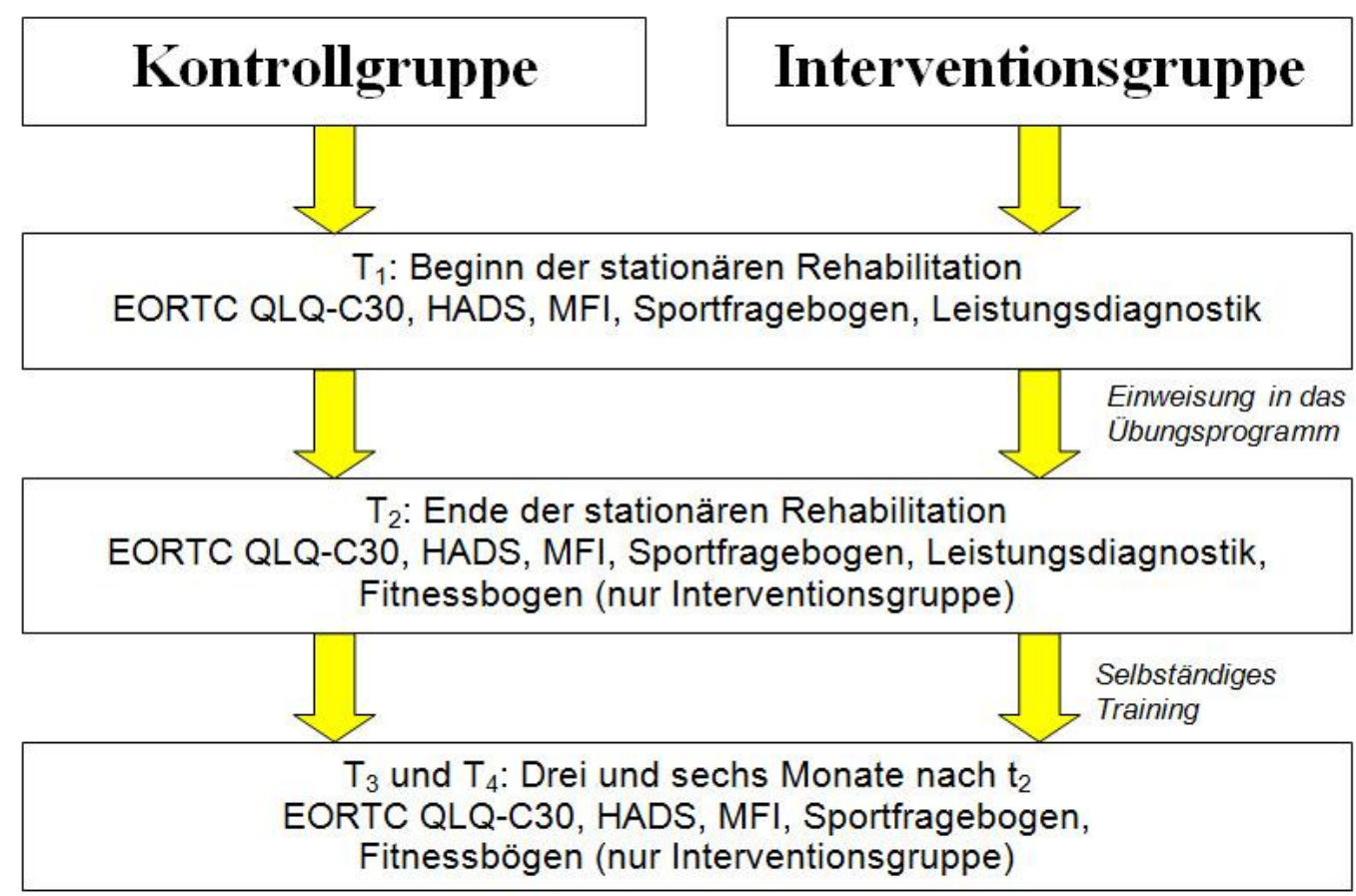

Abbildung 8: Ablauf der Untersuchung (Elsner von der Malsburg, 2006) 


\subsection{Leistungsdiagnostik}

Um den Leistungsstand eines Einzelnen zu Beginn seines Trainings ermitteln zu können, gibt es verschiedene Arten der Leistungsdiagnostik. Diese dient im Allgemeinen dazu, ein Ausgangsniveau zu bestimmen und nach einem Trainingszyklus, Aussagen zur Wirksamkeit und Effektivität des Trainings machen zu können. Besonders wichtig ist es, das Ausgangsniveau eines Trainierenden zu kennen, um so den genauen Trainingsumfang zu bestimmen. Im weiteren Verlauf wird die Belastungssteigerung immer über den Umfang realisiert. Das bedeutet, bei gleich bleibender Intensität (z.B. Geschwindigkeit oder Last) wird der Trainingsumfang der bisherige Belastungen erhöht (Hüter et al., 2005). Im Bezug auf das in der Studie durchgeführten Übungsprogramm bedeutet das, dass die Last oder hier der Widerstand des Terabandes gleich bleibt, der Umfang, also die Wiederholungszahl (z.B. von $2 \times 7$ auf $2 \times 15$ oder $3 \times 20$ Wiederholungen) gesteigert wird.

\subsection{Trainingsadaptation}

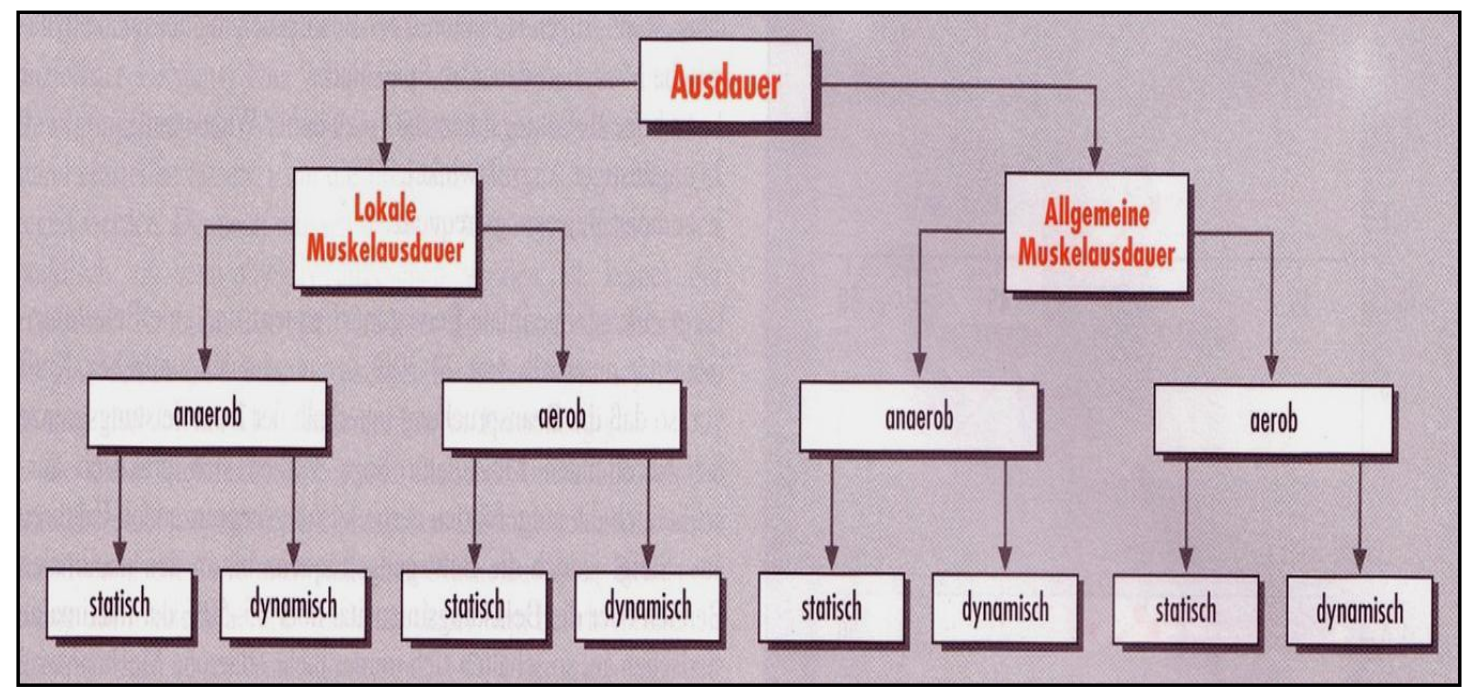

Abbildung 9: Übersicht über die lokale und allgemeine Ausdauer (Hollmann W., 1995)

Wie in Abbildung 9 dargestellt, unterteilt sich die Ausdauer in die allgemeine und lokale Muskelausdauer. In dem Übungsprogramm handelt es sich um ein Training ausschließlich im Bereich der aeroben, allgemeinen und lokalen Muskelausdauer. Hierbei zielen die neun Kräftigungsübungen des Übungsprogramms auf die lokale Muskelausdauer. Dies hat zur Folge, dass die Muskulatur sich der gesteigerten Anforderung anpasst. Auf der 2. Stufe des Stufenmodells zum Muskeltraining kommt es durch das lokale Muskelkraftausdauertraining zu einer Verbesserung des intrazellulären Sauerstoffangebotes, zu einer Erhö- 
hung der Kapazität des mitochondrialen Stoffwechsels und einer Vergrößerung des lokaIen Kohlenhydratdepots (Froböse et al., 2010). Die 3.Stufe des Stufenmodells spielt ebenfalls eine wichtige Rolle in der rehabilitativen Behandlung der tumorassoziierten Fatigue. Durch das Muskelaufbautraining wird eine Hypertrophie, d.h. eine Erhöhung der Muskelmasse und des Muskelquerschnitts und eine Einlagerung von Proteinen erreicht. Dadurch werden durch verminderte Reizwirkung bedingte Atrophien beseitigt. Das wirksamste Training zur Muskelfaserbeeinflussung (schnelle Muskelfasern oder Typ-II-Fasern und langsame Fasern oder Typ-I-Fasern) sind leichte Kontraktionen großer Muskelgruppen (Gehen, Laufen, Radfahren). Hiermit lässt sich die Stoffwechselkapazität um das Drei- bis Vierfache verbessern. Das gilt für beide Muskelfasertypen. Zu berücksichtigen ist dabei jedoch, das Typ-II-Fasern erst bei mittleren bis intensiven Beanspruchungsintensitäten, deren Dauer mehr als 30 Minuten betragen, rekrutiert werden (Hollmann et al., 2000).

Das Walkingprogramm zielt auf die allgemeine Muskelausdauer ab. Dadurch wird die körperliche Leistungsfähigkeit verbessert und degenerativen Herz-Kreislauferkrankungen vorgebeugt. Doch auch das Immunsystem wird entschieden gestärkt.

Durch ein Ausdauertraining wird darüber hinaus die allgemeine Belastungsverträglichkeit und Trainierbarkeit im gesamten Organismus erhöht. Als Folge davon laufen alle regenerativen Prozesse während oder im Anschluss an ein Training beschleunigt ab (Froböse et al., 2010).

Zur Messung der allgemeinen Ausdauer wurden der 6 Min. Gehtest und der PWC- Fahrradergometertest gewählt, die lokale Muskelkraft wurde isometrisch mittels einer Kraftmessdose ermittelt.

\subsection{Min. Gehtest}

Der 6-Min. Gehtest ist ein häufig verwendeter Ausdauertest. Hierbei wird die Gehstrecke ermittelt, die der Patient in sechs Minuten auf ebenem Terrain zurücklegen kann. Der Patient wird vom Therapeuten angeleitet, so weit wie möglich zu gehen, wobei Pausen und Tempowechsel erlaubt sind. Der Patient sollte am Ende des Tests das Gefühl haben, sich angestrengt zu haben. Der Puls wird vor und direkt nach der Belastung und weitere zwei Minuten später gemessen, um einen Eindruck über die Belastbarkeit des kardiopulmonalen Systems zu erhalten. Enright und Sherrill haben in ihrer Studie über Berechnungen des 6 Min. Gehtests für gesunde Frauen mit einem Durchschnittsalter von 59,5 Jahren eine Gehstrecke von 494m ausgemacht (Enright \& Sherrill, 1998). 
Der Test wurde für die vorliegende Studie ausgewählt, da er leicht durchzuführen ist und Ähnlichkeiten zu den Aktivitäten des täglichen Lebens aufweist. Es ist ein Test zur Messung der submaximalen aeroben Ausdauerleistungsfähigkeit. Haass et al. schrieben, dass die Reproduzierbarkeit der Wegstrecke bei einzelnen Patienten bei einer intraindividuellen Varianz von < 5\% lag (Haass et al., 2000). Weiter schrieben Haass et al., dass für den $6-$ Minuten-Gehtest bei kardialen Leistungsminderungen die selben Einschränkungen gelten, wie für eine Spiroergometrie (Haass et al., 2000). Die Sauerstoffaufnahme während eines 6-Minuten-Gehtest entspricht ca. 60-70\% der $\mathrm{VO}_{2 \max }$.

\subsection{PWC Fahrradergometrie}

Grundsätzlich lässt sich ein Fahrradergometertest maximal, bis zur Erschöpfung der Testperson, oder submaximal, bis zu einer annähernden Erschöpfung, durchführen. Um extreme körperliche Belastungen zu vermeiden, wird im Fitness- und Breitensportbereich, sowie in der Rehabilitation ausschließlich submaximal getestet. Die mögliche Aussage zur Leistungsfähigkeit ist dennoch ausreichend genug.

Das Testprotokoll des PWC sah in dieser Studie wie folgt aus:

- Beginn mit 25 Watt

- Steigerung der Belastung stufenweise um 25 Watt alle 3 Minuten

- Belastungsende bei Erreichen des Zielpulses, hier also altersabhängig (110/130 $\min ^{-1}$ )

- Lockeres Ausfahren bei 50 Watt über 4 Minuten, Pulsmessung nach 2 und 4 Minuten

Bei Auftreten folgender Kriterien wurde der Test sofort abgebrochen:

- Unwohlsein der Testperson (Schwindel, starke Müdigkeit, Erschöpfung, Schmerzen)

- Stark abfallende Herzfrequenz

- Stark absinkender Blutdruck

- Zu schnell steigender Blutdruck 
Durch die eingehende Voruntersuchung konnte das Risiko der vorangegangenen Kriterien jedoch deutlich minimiert werden.

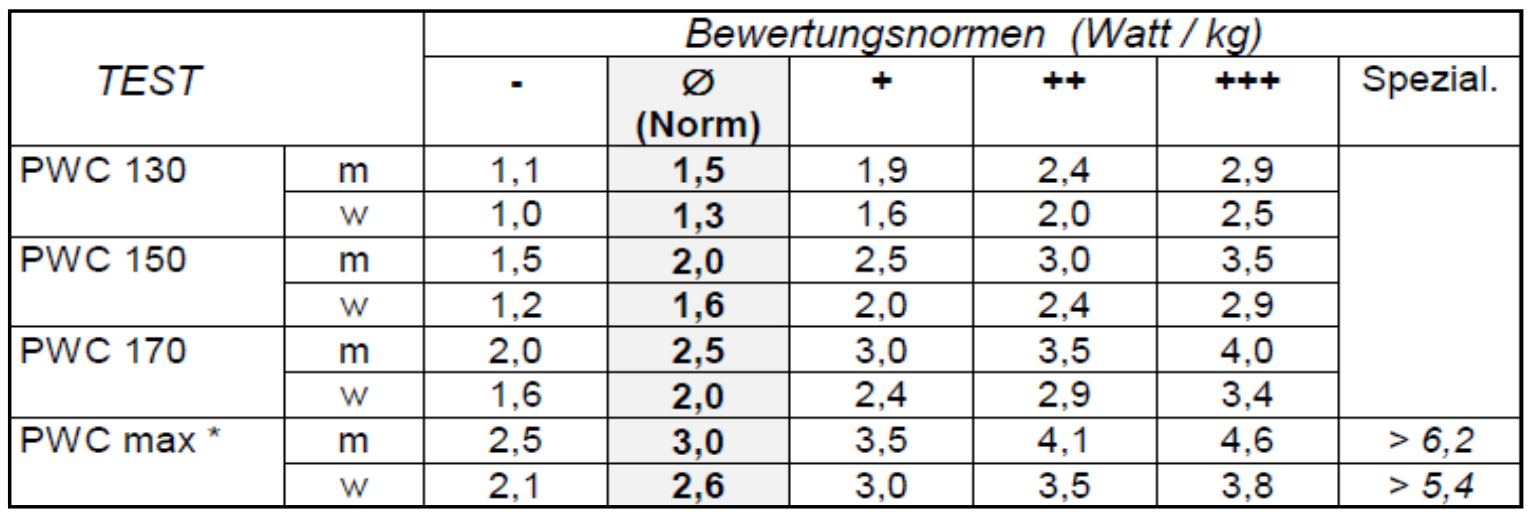

Abbildung 10: Bewertungsnormen des PWC-Tests ( (Stemper, 1988).

Die Abbildung 10 zeigt die relativen Normbelastungswerte in Watt/Kg Körpermasse. Während eines rehabilitativen Trainings nimmt die absolute PWC 170 in Watt und die relative PWC 170 in Watt/Kg Körpermasse zu (Mellerowicz, 1975). Die von der Person erbrachte Ergometerleistung wird mit dem Sollwert verglichen. Abweichungen von mehr als $20 \%$ nach unten werden in einer betriebsärztlichen Untersuchung als nicht normal klassifiziert (Hofmann et al., 2004). Eine genaue Beschreibung der Ergometrie (Mellerowicz, 1975) und eine Tabelle der Leistungsbewertung nach Reuterer befindet sich in der Anlage dieser Arbeit (Hofmann et al., 2004).

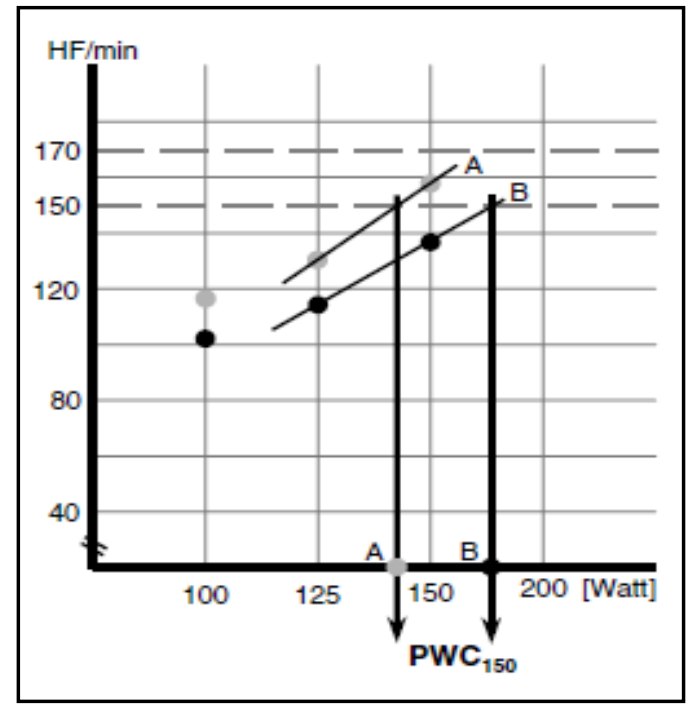

Abbildung 11: Beispiel für die graphische Ermittlung des PWC 150 durch Interpolation bei Über- bzw. Unterschreitung einer Herzschlagfrequenz von 150/min (Hofmann et al., 2004). 


\subsection{Messung der Muskelkraft}

Zur Planung von Trainingsmaßnahmen in der Rehabilitation spielt die Messung der Muskelkraft eine wichtige Rolle, da sie Grundlagen zur Trainingssteuerung und dem Erreichen der Therapieziele dient.

In der Studie wurde zur Messung der isometrischen Muskelkraft ein Kraftmessgerät der Firma Mecha Tronic verwendet. Es handelt sich dabei um ein semi-objektives und apparatives Messverfahren zur Ermittlung der isometrischen Muskelkraft.

Im Gegensatz zur dynamischen, konzentrischen oder exzentrischen, Muskelkontraktion ist die isometrische Muskelkontraktion eine statische Muskelanspannung. Das bedeutet, dass dem Muskel zur Bestimmung der Kraft ein unüberwindbarer Widerstand entgegengesetzt werden muss. Bei der Kraftmessung handelt es sich um eine semi-objektive Messung, da die Patientinnen das Ergebnis teilweise selbst beeinflussen können. Dies geschieht beispielsweise, wenn die Patientinnen im Schulterbereich noch Schmerzen oder Bewegungseinschränkungen durch die Operation aufweisen. Dennoch handelt es sich um zuverlässige und valide Daten, da die Reproduzierbarkeit gegeben ist und durch die Kraftmessung auch die Wirksamkeit der rehabilitativen Behandlungen überprüft werden soll.

Gemessen wurden in der Studie jeweils die Muskelgruppe der Knieextensoren und die Muskelgruppe der Ellenbogenflexoren. Die einzelnen zu den Muskelgruppen gehörenden Muskeln sind in Tabelle 4 aufgeführt.

\begin{tabular}{|c|c|c|}
\hline & Bewegung & Hauptmuskel \\
\hline Kniegelenk & $\begin{array}{c}\text { Extensionsgruppe } \\
\text { (Kniestrecker) }\end{array}$ & 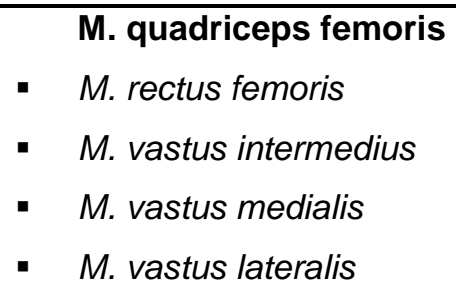 \\
\hline Ellbogengelenk & $\begin{array}{c}\text { Flexionsguppe } \\
\text { (Ellenbogenbeuger) }\end{array}$ & $\begin{array}{l}\text { - } \text { M. Bizeps brachii } \\
\text { - } \quad \text { M. brachioradialis } \\
\text { - } \text { M. brachialis }\end{array}$ \\
\hline
\end{tabular}

Tabelle 4: Muskeln und Muskelgruppen

Mit der Zugehörigkeit einzelner Muskeln zu einer gesamten Muskelgruppe, können auch die Fasertypen (Typ-I-Fasern oder Typ-II-Fasern) variieren. Jedoch sind alle Muskelfasertypen hypertrophiefähig (Hollmann et al., 2000). Langsame Fasern hypertrophieren jedoch 
langsamer als die schnellen Fasern. Der hormonellen Steuerung kommt bei den Hyperund Atrophievorgängen sicherlich eine wesentliche Bedeutung zu. Verschiedene Rückkopplungsmechanismen und Schleifensysteme stimmen externe und interne Faktoren wie muskuläre Aktivität, Ernährung und psychische Aktivität aufeinander ab (Hollmann et al., 2000). Das Grundschema der kybernetischen neuroendokrinen Kontrolle ist in Abbildung 12 dargestellt.

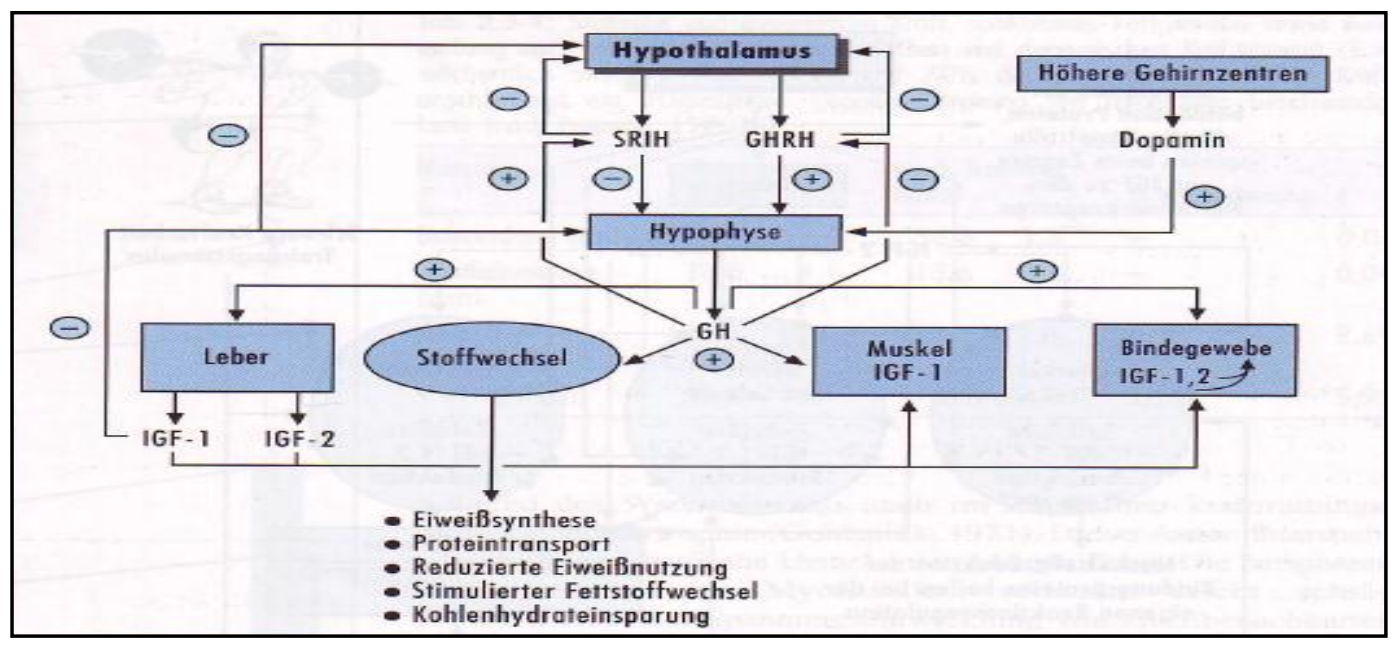

Abbildung 12: Grundschema der kybernetischen neuroendokrinen Kontrolle (Hollmann \& Hettinger).

Neben der neuroendokrinen Kontrolle ändert sich aber auch die Koordination als weitere zentralnervöse Schnittstelle und Teil der motorischen Hauptbeanspruchungsformen. Da alle Muskelgruppen des Körpers auf der Großhirnrinde des Frontallappens repräsentiert sind, basieren willkürliche Bewegungen auf einer Integration des motorischen und des sensorischen Systems. Das bedeutet, dass die Koordination auch als Zusammenwirken von Zentralnervensystem und Skelettmuskulatur verstanden werden kann (Hollmann et al., 2000).

Zur Messung der isometrischen Muskelkraft gehörten in diesem Versuchsaufbau zusätzlich noch:

- Ein höhenverstellbarer Seilzug als Widerlager des Zugapparates

- Eine Beinschlinge, eine Handschlinge, Karabinerhaken und ein Hocker

- Eine höhenverstellbare Therapiebank

- Die Messtechnik, bestehend aus dem digitalen Handgerät, dem Kraftsensor und einem PC mit Messsoftware 
Der DIGIMAX ist ein digitales Messgerät mit dem die Kraftmessung über eine Kraftmessdose erfolgt. Angeschlossen an einen PC kann über die Messsoftware die Messkurve und die erreichte Maximalkraft in Newton abgelesen werden.

Der Messablauf für die Messung der isometrischen Maximalkraft wird im Folgenden kurz erläutert:

1. Die Personendaten werden in die Messsoftware eingetragen. Fehlende Daten werden später durch den Untersuchungsleiter ergänzt.

2. Der Untersuchungsleiter erklärt allen Testteilnehmern den Messablauf und führt die jeweilige Messposition vor.

3. Der Proband nimmt die jeweilige Messposition ein. Die Länge der Fixierungsgurte, an denen der Messgriff bzw. die Messschlinge befestigt wird, ist der Körpergröße bzw. der Länge der Extremitäten angepasst. Bei Bedarf wird die höhenverstellbare Untersuchungsbank angepasst. Zu beachten ist, dass sowohl im Ellbogengelenk/Kniegelenk als auch zwischen Unterarm/Unterschenkel und Messschlinge/Messgriff ein rechter Winkel zu messen ist (vgl. Abbildungen 13 und 14).

4. Nach einem Probeversuch erfolgt die Messung. Bei der Messung geht der Proband nach der Anweisung "Achtung" in Vorspannung. Nach dem Kommando "Start" erfolgt die vier Sekunden lange Messung, die durch das Kommando "Stopp“ beendet wird. Parallel zur Messung wird der Kraftverlauf auf dem Bildschirm des PCs mittels eines Graphen bildlich dargestellt. Bei erfolgreicher Kraftmessung muss die Messung nicht wiederholt werden und die Messdaten werden im Computer abgespeichert. Wichtig ist, dass die Brustkrebspatientinnen, deren OP erst wenige Wochen zurückliegt, vor der Messung des Ellbogengelenks auf der betroffenen Seite, angehalten werden, nicht zu ruckartig an dem Messgriff zu ziehen. Das Risiko von Verletzungen oder die Entstehung eines Lymphödems sollen damit ausgeschlossen werden.

5. Nach erfolgreicher Messung wird die Körperseite gewechselt.

6. Nachdem bei allen Probanden die Messung in der ersten Messposition erfolgt ist, wird der Messplatz umgebaut und es erfolgt die nächste Messung auf beiden Seiten. 
Die Abbildungen 13 und 14 stellen die beiden Kraftmesspositionen in stark vereinfachter Form dar.

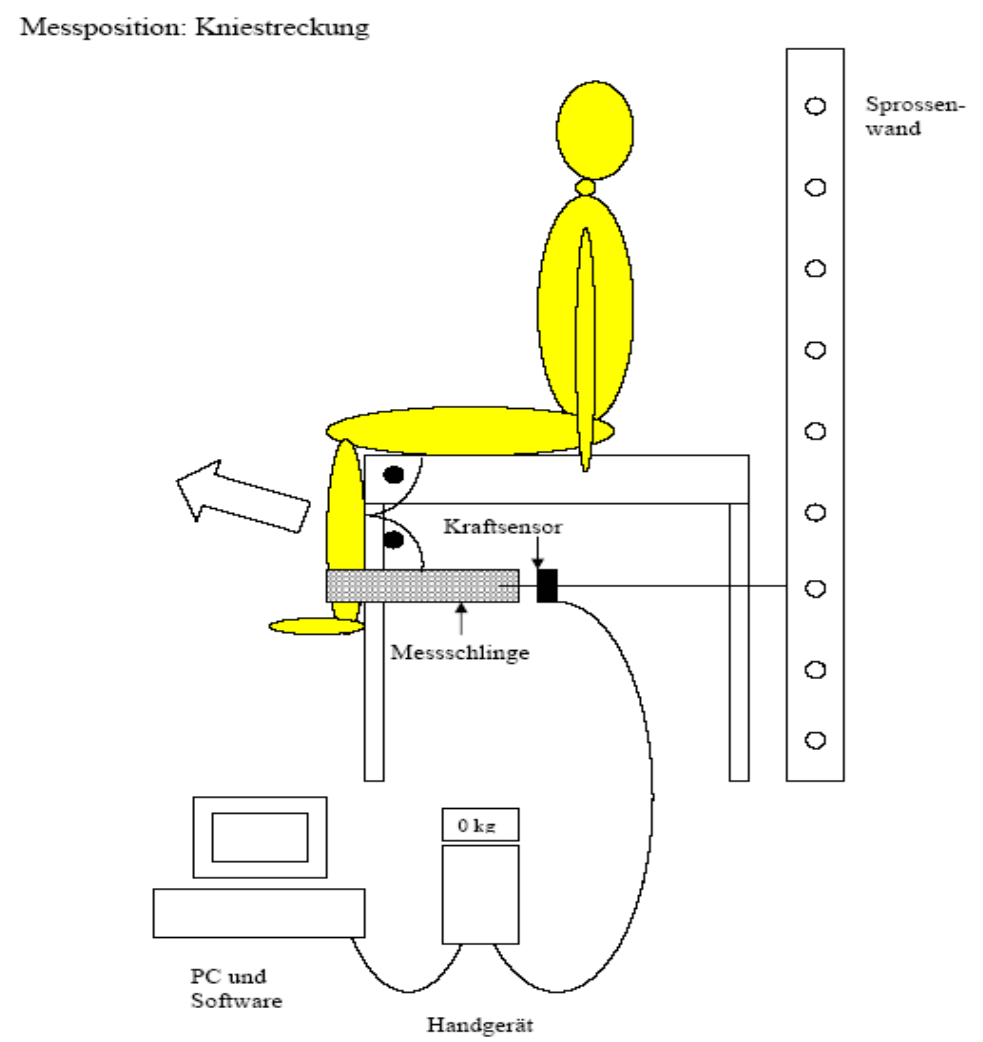

Abbildung 13: Messposition Kniestreckung (Elsner von der Malsburg, 2006). 


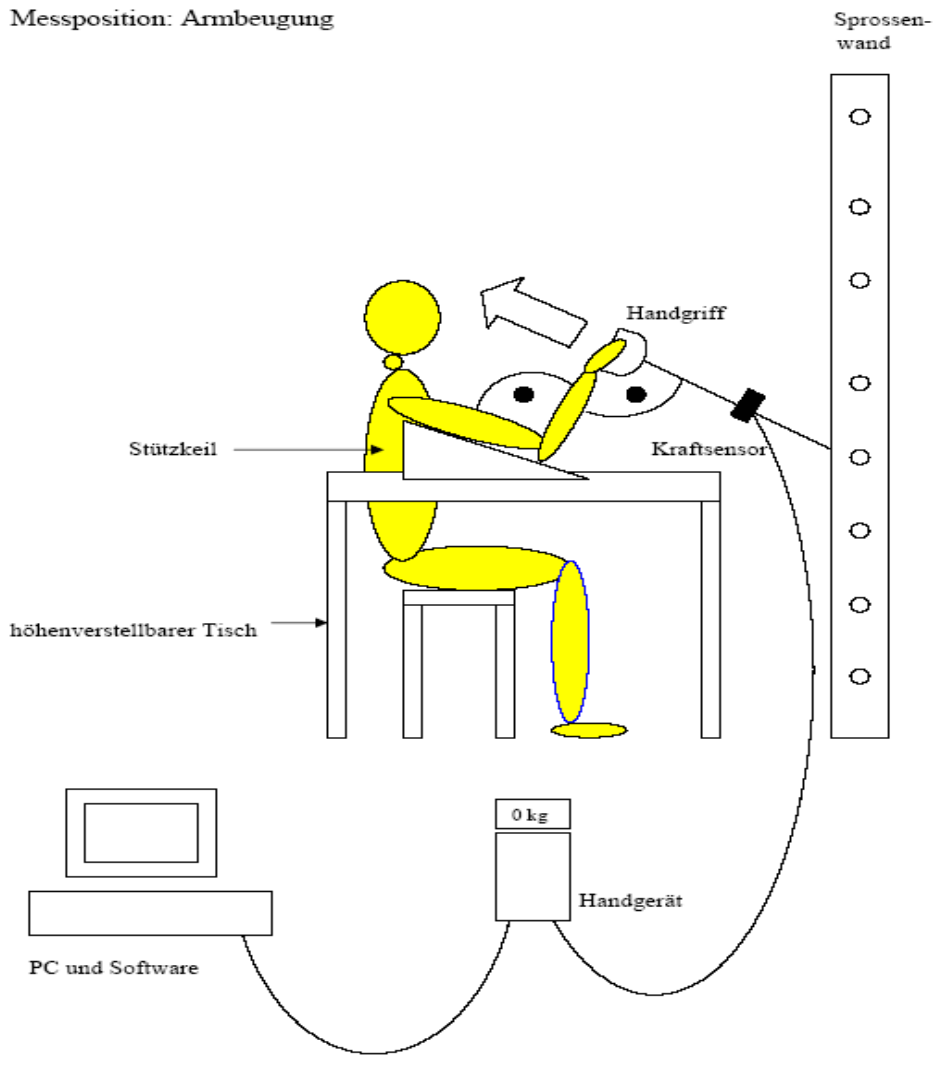

Abbildung 14: Messposition Armbeuger (Elsner von der Malsburg, 2006).

\subsection{Statistische Auswertung der Ergebnisse}

Die statistische Auswertung der Fragebögen erfolgte mit Hilfe der Programme SPSS 17.0 für Windows und Graph Pad Prism 4.0. Zur Auswertung des HADS und des EORTC QLQ C-30 wurden die jeweiligen Scoring Guidelines verwendet. Die Kraftmessungen und Kraftänderungen wurden mit Hilfe der Digimax Software ermittelt. Die Signifikanzprüfungen wurden mit dem t-Test durchgeführt, mit einer Irrtumswahrscheinlichkeit von unter 5\%, d.h. die Signifikanzgrenze lag bei: Signifikanz für $P \leq 0,05$. 


\section{Ergebnisse}

\subsection{Soziodemographische Daten}

Wie im Abb.15a dargestellt, betrug das Durchschnittsalter der Patientinnen der Kontroll-

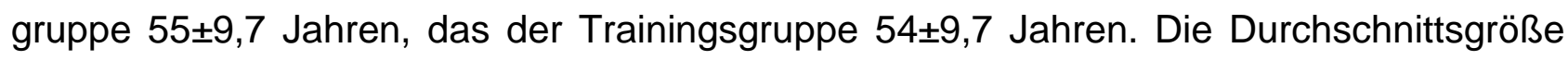
der Patientinnen der Kontrollgruppe, dargestellt in Abb.15c, lag bei 165,8 $\pm 6,2 \mathrm{~cm}$, das Durchschnittsgewicht bei $71,2 \pm 14,6 \mathrm{Kg}$. Die Trainingsgruppe hatte eine Durchschnittsgrö-

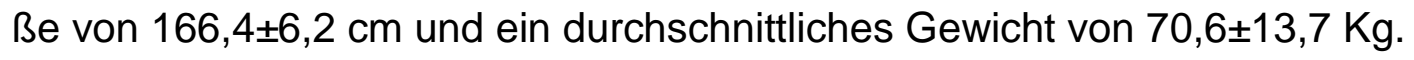

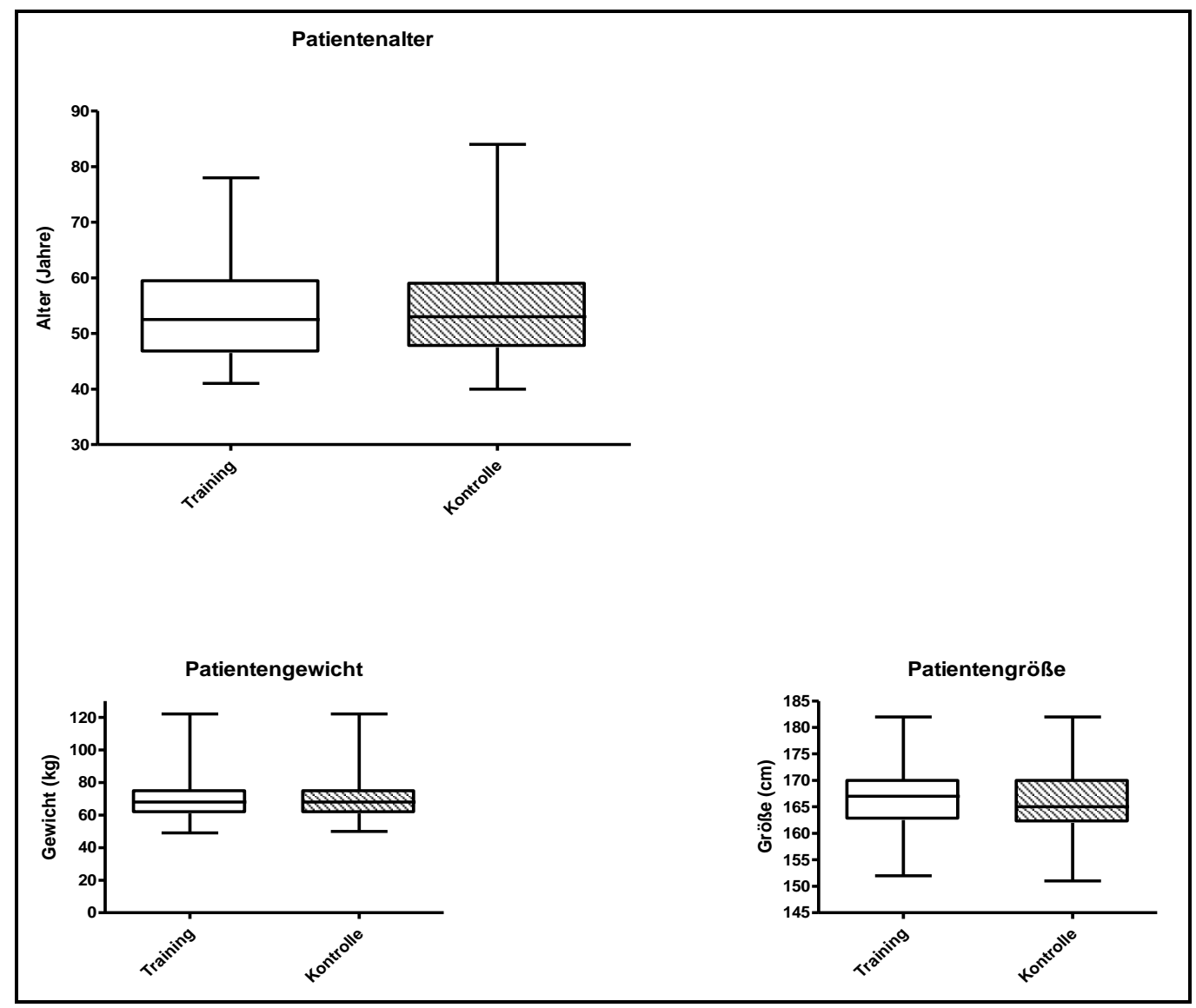

Abbildung 15 a-c: Graphische Darstellung von Alter, Gewicht und Größe der Patientinnen beider Gruppen.

In Tabelle 5 sind die jüngste und die älteste Patientinnen, sowie das Durchschnittsalter der teilnehmenden Patientinnen dargestellt. Des Weiteren zeigt die Tabelle verschiedene Altersgruppen und die Anzahl der Patientinnen dieser Altersgruppen. 


\begin{tabular}{|l|l|l|}
\hline Patientendaten & Trainingsgruppe & Kontrollgruppe \\
\hline Jüngste Pat. (Jahre) & 41 & 40 \\
\hline Älteste Pat. (Jahre) & 78 & 84 \\
\hline$\varnothing$ Alter der Pat. (Jahre) & $54 \pm 9,7$ & $55 \pm 9,7$ \\
\hline -30 Jahre (Anzahl) & 0 & 0 \\
\hline 30-45 Jahre (Anzahl) & 9 & 10 \\
\hline 46-60 Jahre (Anzahl) & 26 & 40 \\
\hline$>60$ Jahre (Anzahl) & 11 & 15 \\
\hline
\end{tabular}

Tabelle 5: Angaben beider Studiengruppen über Alter und Anzahl der Patientinnen in den unterschiedlichen Altersgruppen.

In Tabelle 6 sind Maximal- und Minimalgröße und Gewicht, sowie die Durchschnittswerte beider Gruppen angegeben.

\begin{tabular}{|l|l|l|}
\hline Patientendaten & Trainingsgruppe & Kontrollgruppe \\
\hline Pat. Größe Max. $(\mathrm{cm})$ & 182 & 182 \\
\hline Pat. Größe Min. $(\mathrm{cm})$ & 152 & 151 \\
\hline$\varnothing$ Größe $(\mathrm{cm})$ & 166,4 & 165,8 \\
\hline Pat. Gewicht Max. $(\mathrm{Kg})$ & 122 & 122 \\
\hline Pat. Gewicht Min. $(\mathrm{Kg})$ & 49 & 50 \\
\hline$\varnothing$ Gewicht $(\mathrm{Kg})$ & 70,63 & 71,22 \\
\hline
\end{tabular}

Tabelle 6: Angaben beider Studiengruppen über Größe und Gewicht.

\subsubsection{Berufliche Situation}

49,2\% der Patientinnen der Trainingsgruppe waren zur Zeit der Studienteilnahme in Teilzeitbeschäftigung. 23,7\% waren Ganztagsbeschäftigt, 11,9\% übten eine Tätigkeit als Hausfrauen aus. 15,3\% waren bereits Rentnerinnen (siehe Tabelle 7).

\begin{tabular}{|l|r|r|}
\hline & Häufigkeit & Gültige Prozente \\
\hline Hausfrau & 7 & 11,9 \\
\hline Ganztagsbeschäftigung & 14 & 23,7 \\
\hline Teilzeitbeschäftigung & 29 & 49,2 \\
\hline Rentnerin/Ruhestand & 9 & 15,3 \\
\hline Gesamt & 59 & 100,0 \\
\hline
\end{tabular}

Tabelle 7: Berufliche Situation der Trainingsgruppe zum Messzeitpunkt T1. 
Die Patientinnen der Kontrollgruppe waren zu 33,3\% Teilzeitbeschäftigt, 29,2\% gingen einer Ganztagsbeschäftigung nach. Als Hausfrauen tätig waren 4,2\%, 27,1\% waren Rentnerinnen. Keine Angaben zu ihrer beruflichen Situation machten 6,3\% (siehe Tabelle 8).

\begin{tabular}{|l|r|r|}
\hline & Häufigkeit & Gültige Prozente \\
\hline Hausfrau & 2 & 4,2 \\
\hline Ganztagsbeschäftigung & 14 & 29,2 \\
\hline Teilzeitbeschäftigung & 16 & 33,3 \\
\hline Rentnerin/Ruhestand & 13 & 27,1 \\
\hline keine Angabe & 3 & 6,3 \\
\hline Gesamt & 48 & 100,0 \\
\hline
\end{tabular}

Tabelle 8: Berufliche Situation der Kontrollgruppe zum Messzeitpunkt T1.

\subsubsection{Patientenanzahl und Verlauf}

Von den insgesamt 447 mittels LASA-Bogen gescreenten Patientinnen, gaben 149 Patientinnen einen Fatiguescore von 3 oder weniger an. 156 Patientinnen markierten auf der Skala einen Fatiguescore von 4-6, 111 Patientinnen einen Score von 7-8. 31 Patientinnen lagen bei dem Screening bei einem Wert über 8. Das bedeutet, dass 298 Patientinnen $(66,7 \%)$ der Gesamtanzahl der Patientinnen für eine Studienteilnahme geeignet waren (siehe Tabelle 9).

\begin{tabular}{|l|l|l|}
\hline Fatiguescore (0-10) & Patientenanzahl & Anzahl in \% \\
\hline $0-3$ & 149 & 33,3 \\
\hline $4-6$ & 156 & 34,9 \\
\hline $7-8$ & 111 & 24,8 \\
\hline$>8$ & 31 & 7,0 \\
\hline Gesamt: & 447 & $100 \%$ \\
\hline
\end{tabular}

Tabelle 9: Anzahl der Patientinnen in den einzelnen Scores des Fatigue-LASA.

Zum Messzeitpunkt T1 lag die Anzahl der Patientinnen der Trainingsgruppe bei 70 Teilnehmerinnen, die der Kontrollgruppe bei 56 (siehe Abb.16a und b, sowie Tabelle10). Zum Messzeitpunkt T2 waren in der Trainingsgruppe noch 48 Patientinnen dabei, in der Kontrollgruppe noch 50 Patientinnen. An T3 nahmen noch 41 Patientinnen der Trainingsgruppe an der Studie teil, in der Kontrollgruppe waren noch 40 Teilnehmerinnen dabei. Am 
letzten Messzeitpunkt T4 wurden in der Trainingsgruppe noch 40 Patientinnen gezählt, in der Kontrollgruppe 34 Patientinnen.

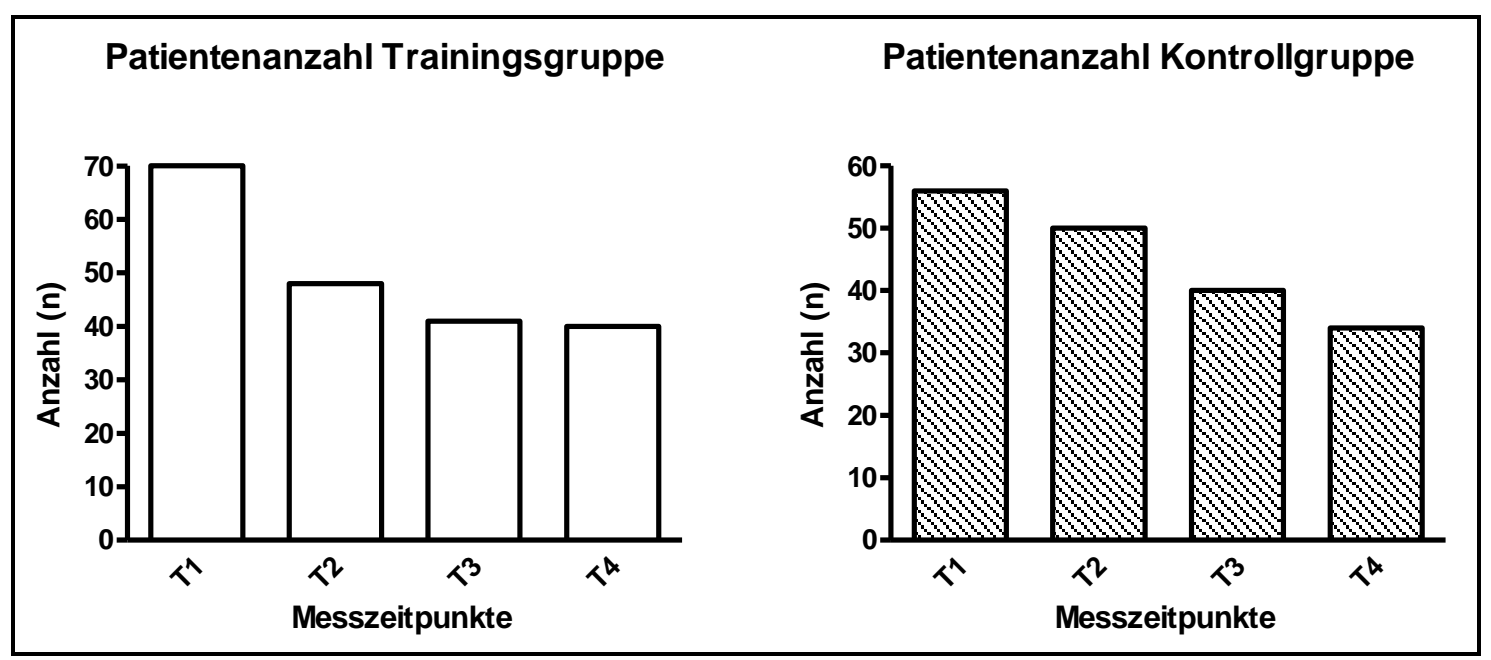

Abbildung 16 a und b: Patientinnenanzahl an den einzelnen Messzeitpunkten beider Gruppen.

\begin{tabular}{|l|l|l|l|l|}
\hline Patientenanzahl & T1 & T2 & T3 & T4 \\
\hline Trainingsgruppe & 70 & 48 & 41 & 40 \\
\hline Kontrollgruppe & 56 & 50 & 40 & 34 \\
\hline
\end{tabular}

Tabelle 10: Patientinnenanzahl an den einzelnen Messzeitpunkten beider Gruppen.

Rechnet man die Teilnehmerinnenzahlen in prozentuale Werte an den einzelnen Messzeitpunkten um, kommt man zu folgendem Ergebnis:

\begin{tabular}{|l|l|l|}
\hline Messzeitpunkt & Trainingsgruppe (\%) & Kontrollgruppe (\%) \\
\hline T2 & 68,6 & 89,3 \\
\hline T3 & 58,6 & 71,4 \\
\hline T4 & 57,1 & 60,7 \\
\hline
\end{tabular}

Tabelle 11: Prozentuale Teilnehmerinnenanzahl bezogen auf den Messzeitpunkt T1=100\%.

Das bedeutet, dass in der Trainingsgruppe am Ende der Rehabilitation noch 68,6\% der teilnehmenden Patientinnen für die Messungen zur Verfügung standen. In der Kontrollgruppe waren es noch $89,3 \%$ (siehe Tabelle 11).

Zu den Messzeitpunkten T3 und T4 schickten 58,6 bzw. 57,1\% der Patientinnen der Trainingsgruppe die innen zugesandten Fragebögen und die ausgefüllten Trainingsdokumen- 
tationsbögen wieder zurück. Aus der Kontrollgruppe sendeten zum Messzeitpunkt T3 $71,4 \%$ der Patientinnen die Studienunterlagen wieder zurück, an T4 waren es noch 60,7\% (siehe Tabelle 11).

\subsection{Durchführung des Übungsprogramms}

Wie in Abb. 17a und b und in Tabelle 12 und 13 dargestellt führten die Teilnehmerinnen der Trainingsgruppe die Kraftübungen des Übungsprogramms im ersten Monate nach der Rehabilitation durchschnittlich 7,5-mal durch. Die Dehnübungen wurden im Schnitt 7,4-mal durchgeführt. Im zweiten Monat wurden die Kraftübungen 8,3-mal durchgeführt, die Dehnübungen 8,4-mal. Während des dritten Monats führten die Teilnehmerinnen die Kraftübungen 7,3-mal pro Monat durch, die Dehnübungen wurden 7,4-mal durchgeführt. Im vierten Trainingsmonat übten die Teilnehmerinnen 7,6-mal monatlich die Kraft- und 7,8-mal die Dehnübungen aus. Im fünften Monat trainierten die Teilnehmerinnen die Kraftübungen 7,3-mal monatlich, die Dehnungen 7,4-mal. Im letzen Trainingsmonat wurden die Kraftübungen 6,7-mal im Monat durchgeführt, die Dehnübungen durchschnittlich 6,9-mal. Rechnet man die durchschnittliche Trainingsanzahl der einzelnen Monate zusammen und dividiert das Ergebnis durch vier, bekommt man die Anzahl, wie oft das Übungsprogramm wöchentlich durchgeführt wurde (siehe Tabelle 14). Für die Kraftübungen lag das wöchentliche Training bei 1,85-mal, für die Dehnübungen bei 1,875-mal. Da die Vorgabe lautete, die Übungen mindestens 2-mal wöchentlich durchzuführen, um 100\% des Trainingsumfangs erfüllt zu haben, lagen die Teilnehmerinnen in der Durchführung der Kraftübungen bei $92,5 \%$, in der Durchführung der Dehnübungen bei 93,75\% (siehe Abb. 15a und b; Tabelle 12 und 13).

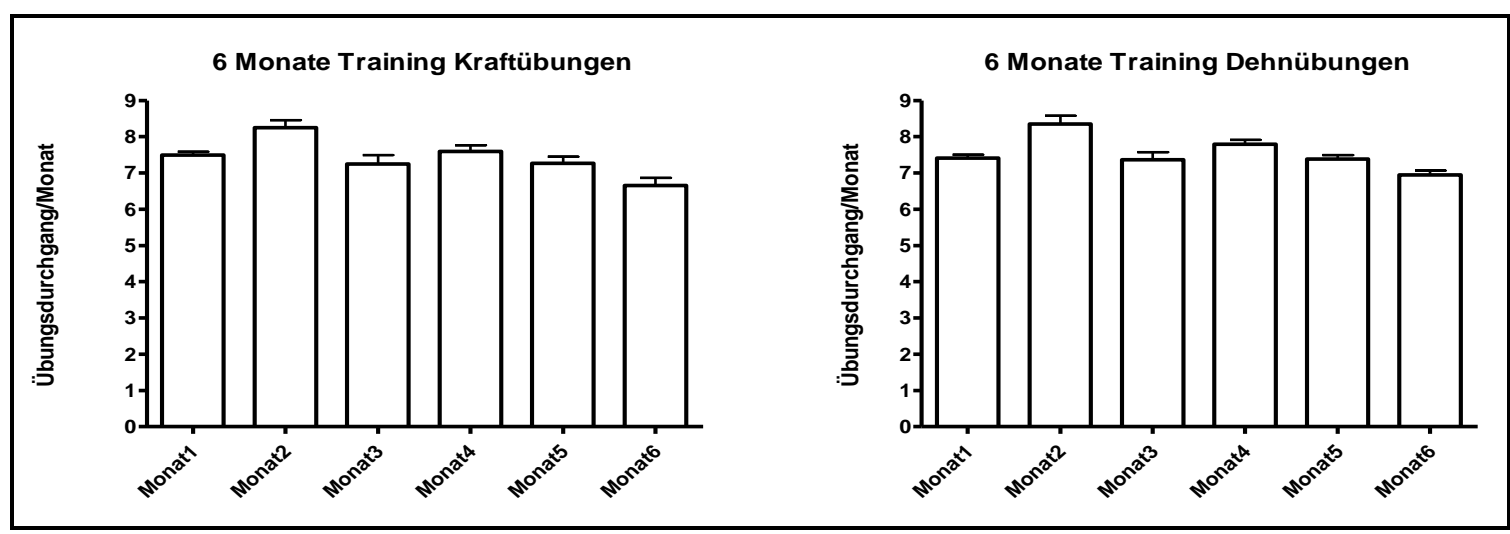

Abbildung 17 a und b: Anzahl der im Monat durchgeführten Kraft- und Dehnübungen beider Gruppen. 


\begin{tabular}{|l|l|l|l|l|l|l|}
\hline Monat & Monat1 & Monat2 & Monat3 & Monat4 & Monat5 & Monat6 \\
\hline Mean & 7,4 & 8,4 & 7,4 & 7,8 & 7,4 & 6,9 \\
\hline SD ( \pm ) & 0,28 & 0,7 & 0,61 & 0,36 & 0,33 & 0,37 \\
\hline
\end{tabular}

Tabelle 12: Anzahl durchgeführter Dehnübungen pro Monat (nach Rehabilitation).

\begin{tabular}{|l|l|l|l|l|l|l|}
\hline Monat & Monat1 & Monat2 & Monat3 & Monat4 & Monat5 & Monat6 \\
\hline Mean & 7,5 & 8,3 & 7,3 & 7,6 & 7,3 & 6,7 \\
\hline SD ( \pm$)$ & 0,29 & 0,62 & 0,73 & 0,52 & 0,55 & 0,61 \\
\hline
\end{tabular}

Tabelle 13: Anzahl durchgeführter Kraftübungen pro Monat (nach Rehabilitation)

\begin{tabular}{|l|l|}
\hline Kraftübungen & Dehnübungen \\
\hline $1,85 \times$ wöchentlich & $1,875 \times$ wöchentlich \\
\hline $92,5 \%(100 \%=2 \times$ wöchentlich $)$ & $93,75 \%(100 \%=2 \times$ wöchentlich $)$ \\
\hline
\end{tabular}

Tabelle 14: Prozentuale Durchführung der Kraft- und Dehnübungen

\subsubsection{Ausdauertraining}

Tabelle 15 zeigt, dass bis zum Messzeitpunkt T3, also in den Monaten 1-3 nach der Rehabilitation, 14 Patientinnen durchschnittlich 4 Stunden pro Monat ein Ausdauertraining durchgeführt haben. 12 Patientinnen führten in dieser Zeit 5-8 Stunden Ausdauertraining pro Monat durch und 9 Patientinnen über 8 Stunden im Monat. Bis zum Messzeitpunkt T4, also in den Monaten 4-6 nach Rehabilitation führten 22 Patientinnen bis zu 4 Stunden Ausdauersport pro Monat durch. 8 Patientinnen brachten es auf 5 bis 8 Stunden Ausdauerbelastung pro Monat und nur 5 Patientinnen trainierten über 8 Stunden monatlich im Ausdauerbereich.

\begin{tabular}{|l|l|l|}
\hline & $\mathrm{N}$ bei T3 & $\mathrm{N}$ bei T4 \\
\hline Unterdurchschnitt $\leq$ 4Std/Mon. & 14 & 22 \\
\hline Durchschnitt 5-8 Std./Mon. & 12 & 8 \\
\hline Überdurchschnitt >8 Std./Mon. & 9 & 5 \\
\hline
\end{tabular}

Tabelle 15: Unter- über- und durchschnittlicher Trainingsumfang und Anzahl der Patientinnen. 
Die Patientinnen der Trainingsgruppe trainierten in den Monaten 1-3 nach der Rehabilitation durchschnittlich 7,58 $\pm 3,91$ Stunden pro Monat. In den Monaten 4-6 nach der Rehabilitation trainierten sie durchschnittlich 5,58 $\pm 4,89$ Stunden pro Monat (siehe Abb.18 und Tabelle 16).

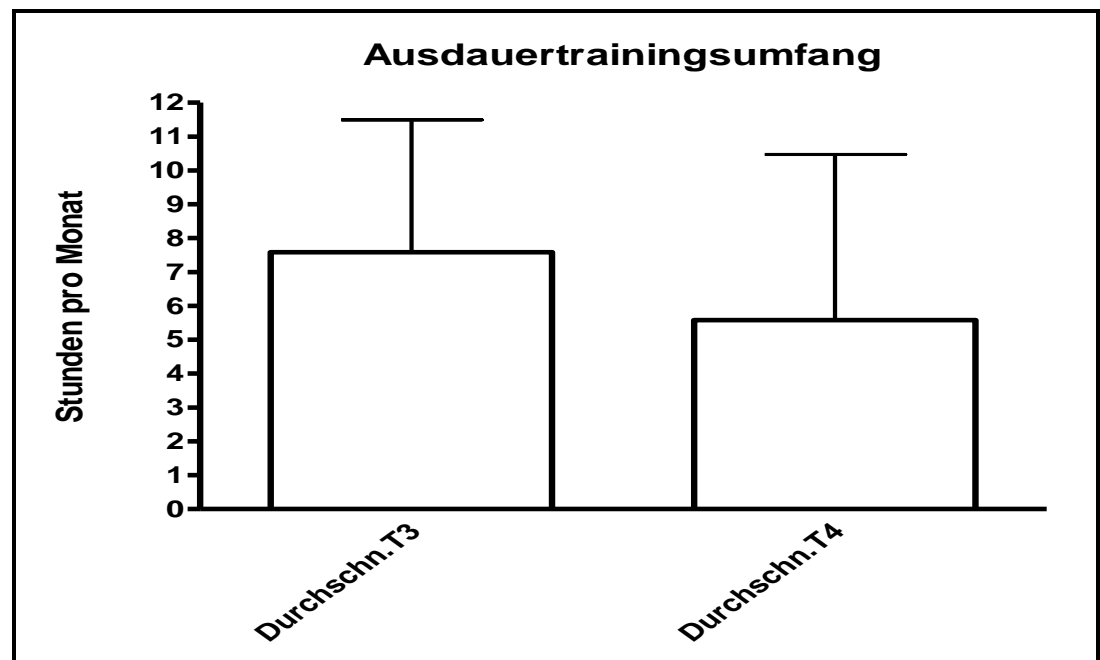

Abbildung 18: Durchschnittlicher Ausdauertrainingsumfang in Std./Monat an den Messzeitpunkten T3 und T4.

\begin{tabular}{|l|l|l|}
\hline & Trainingsschnitt T3 & Trainingsschnitt T4 \\
\hline Mean (Std/M.) & 7,58 & 5,58 \\
\hline SD ( \pm ) & 3,91 & 4,89 \\
\hline
\end{tabular}

Tabelle 16: Trainingsschnitt in Stunden / Monat zu T3 und T4.

\subsection{Behandlungen der Brusterkrankung}

Tabelle 17 zeigt, bei wie viel Prozent der Studienteilnehmerinnen ein operativer Eingriff durchgeführt wurde. Bei 65,3\% der Patientinnen der Trainingsgruppe wurde ein operativer Eingriff an der Brust durchgeführt. Bei einer Patientin (1,4\%) wurde kein operativer Eingriff vorgenommen, 33,3\% der Patientinnen machten keine Angaben, ob eine Operation durchgeführt wurde.

\begin{tabular}{|l|l|r|r|r|}
\hline \multicolumn{2}{|c|}{} & Häufigkeit & \multicolumn{1}{c|}{ Prozent } & Gültige Prozente \\
\hline \multirow{3}{*}{ Gültig } & 1 & 1,4 & 1,4 \\
\cline { 2 - 5 } & nein & 47 & 65,3 & 65,3 \\
\cline { 2 - 5 } & ja & 24 & 33,3 & 33,3 \\
\cline { 2 - 5 } & keine Angabe & 72 & 100,0 & 100,0 \\
\cline { 2 - 5 } & Gesamt & &
\end{tabular}

Tabelle 17: Operative Eingriffe bei den Patientinnen der Trainingsgruppe 
Wie in Abbildung 19 und Tabelle 18 dargestellt, wurden 72,4\% der Patientinnen der Trainingsgruppe brusterhaltend operiert. Bei $23,7 \%$ der Gruppe wurde eine Mastektomie durchgeführt. 2,6\% wurden nicht operiert und 1,3\% machten hierzu keine Angaben.

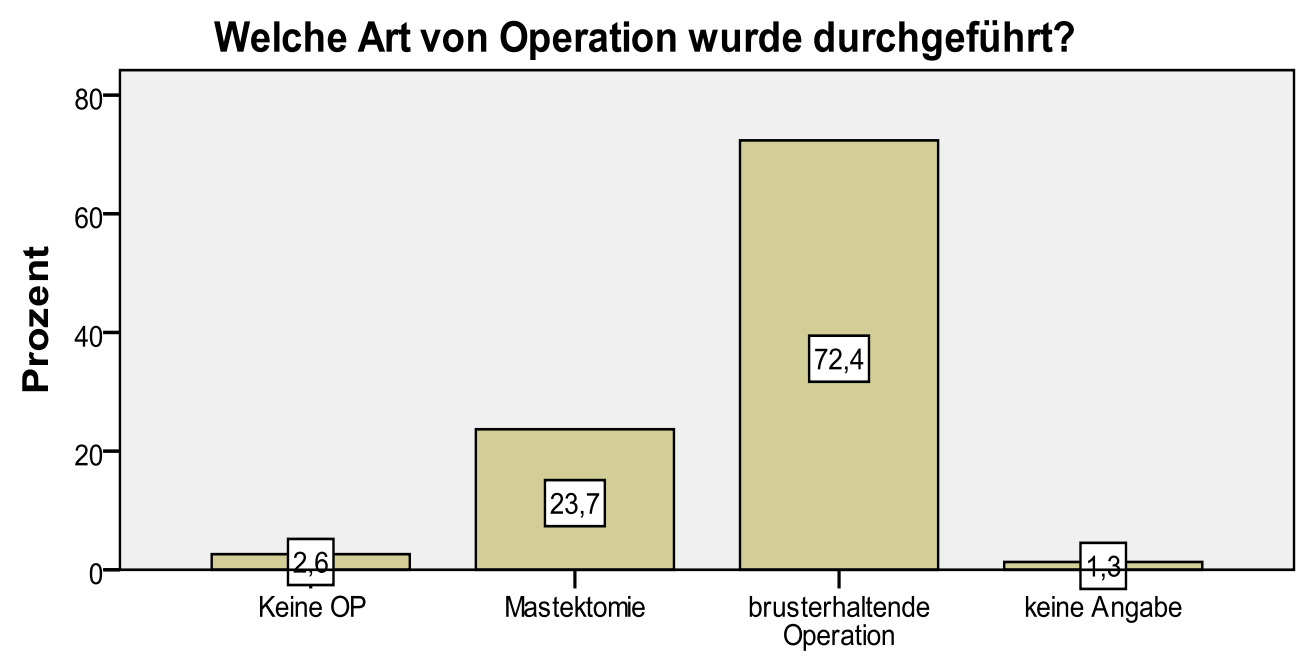

Abbildung 19: Art der durchgeführten Operation bei den Patientinnen der Trainingsgruppe.

\begin{tabular}{|l|l|r|r|}
\hline \multicolumn{2}{|c|}{} & Häufigkeit & Gültige Prozente \\
\hline \multirow{4}{*}{ Gültig } & Keine OP & 2 & 2,6 \\
\cline { 2 - 4 } & Mastektomie & 18 & 23,7 \\
\cline { 2 - 4 } & brusterhaltende Operation & 55 & 72,4 \\
\cline { 2 - 4 } & keine Angabe & 1 & 1,3 \\
\cline { 2 - 4 } & Gesamt & 76 & 100,0 \\
\hline
\end{tabular}

Tabelle 18: Art der durchgeführten Operation in der Trainingsgruppe.

In der Kontrollgruppe gaben 82\% der Patientinnen an, dass ein operativer Eingriff durchgeführt wurde. Bei 4,0\% der befragten Patientinnen wurde keine Operation durchgeführt. $14,0 \%$ der Patientinnen machten keine Angaben zu den operativen Eingriffen (siehe Tabelle 19).

\begin{tabular}{|l|l|r|r|}
\hline \multicolumn{2}{|c|}{} & Häufigkeit & \multicolumn{1}{c|}{ Prozent } \\
\hline \multirow{3}{*}{ Gültig } & nein & 2 & 4,0 \\
\cline { 2 - 4 } & ja & 41 & 82,0 \\
\cline { 2 - 4 } & keine Angabe & 7 & 14,0 \\
\cline { 2 - 4 } & Gesamt & 50 & 100,0 \\
\hline
\end{tabular}

Tabelle 19: Operative Eingriffe bei den Patientinnen der Kontrollgruppe 
In der Kontrollgruppe wurden, wie Abb.20 und Tabelle 20 zeigen, 62\% der Patientinnen brusterhaltend operiert. Bei 18\% wurde eine Mastektomie durchgeführt, 4\% wurden nicht operiert. 16\% der teilnehmenden Patientinnen machten keine Angaben.

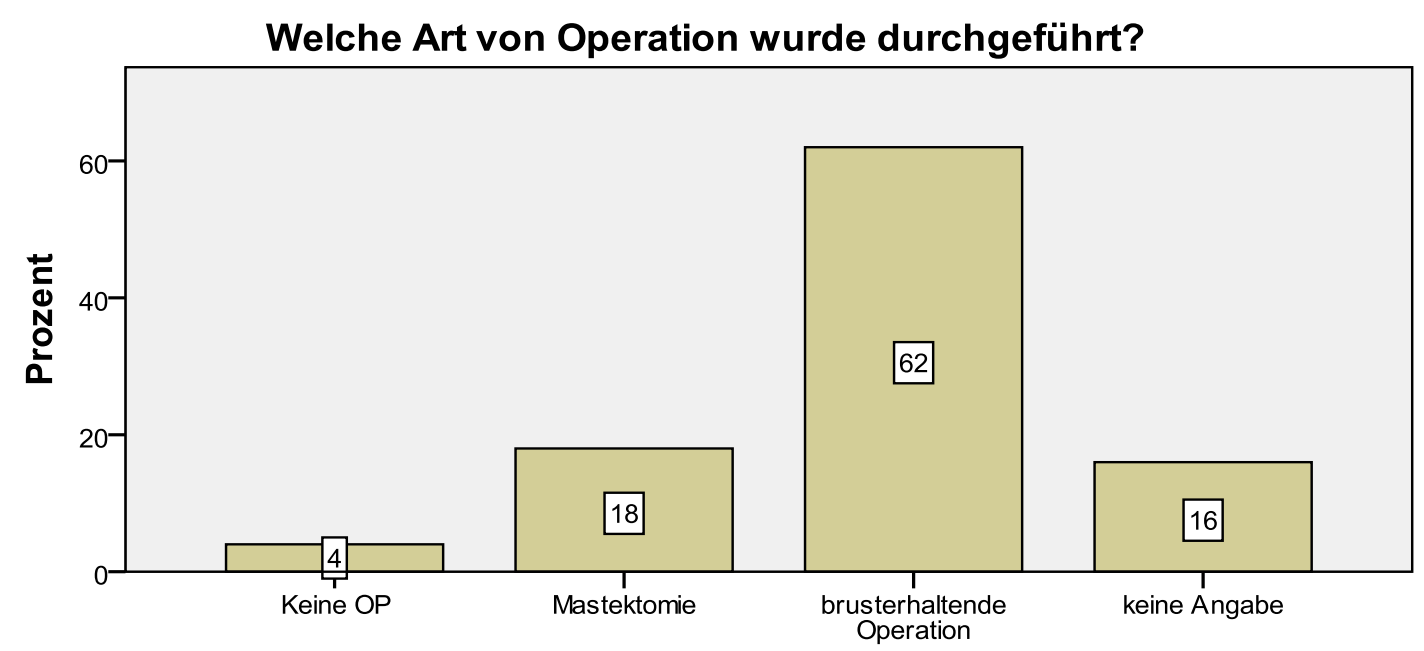

Abbildung 20: Art der durchgeführten Operation bei den Patientinnen der Kontrollgruppe.

\begin{tabular}{|l|r|r|}
\hline & Häufigkeit & Prozent \\
\hline Keine OP & 2 & 4,0 \\
\hline Mastektomie & 9 & 18,0 \\
\hline brusterhaltende Operation & 31 & 62,0 \\
\hline keine Angabe & 8 & 16,0 \\
\hline Gesamt & 50 & 100,0 \\
\hline
\end{tabular}

Tabelle 20: Art der durchgeführten Operation in der Kontrollgruppe.

Die folgenden Fragen nach durchgeführten Hormon- Chemo- und Strahlentherapie beziehen sich alle auf den Zeitraum vor der Rehabilitation und wurde auch nur für diesen Zeitraum dokumentiert und ausgewertet. 
Eine Chemotherapie ist bei $52,6 \%$ der Patientinnen der Trainingsgruppe durchgeführt worden. Bei $31,6 \%$ der Patientinnen wurde keine Chemotherapie durchgeführt. 15,8\% machten hierzu keine Angaben (siehe Tabelle 21 und Abb.21).

\begin{tabular}{|l|l|r|r|}
\hline \multicolumn{2}{|c|}{} & \multicolumn{1}{|c|}{ Häufigkeit } & \multicolumn{1}{c|}{ Prozent } \\
\hline \multirow{4}{*}{ Gültig } & nein & 18 & 31,6 \\
\cline { 2 - 4 } & ja & 30 & 52,6 \\
\cline { 2 - 4 } & keine Angabe & 9 & 15,8 \\
\cline { 2 - 4 } & Gesamt & 57 & 100,0 \\
\hline
\end{tabular}

Tabelle 21: Durchführung einer Chemotherapie in der Trainingsgruppe.

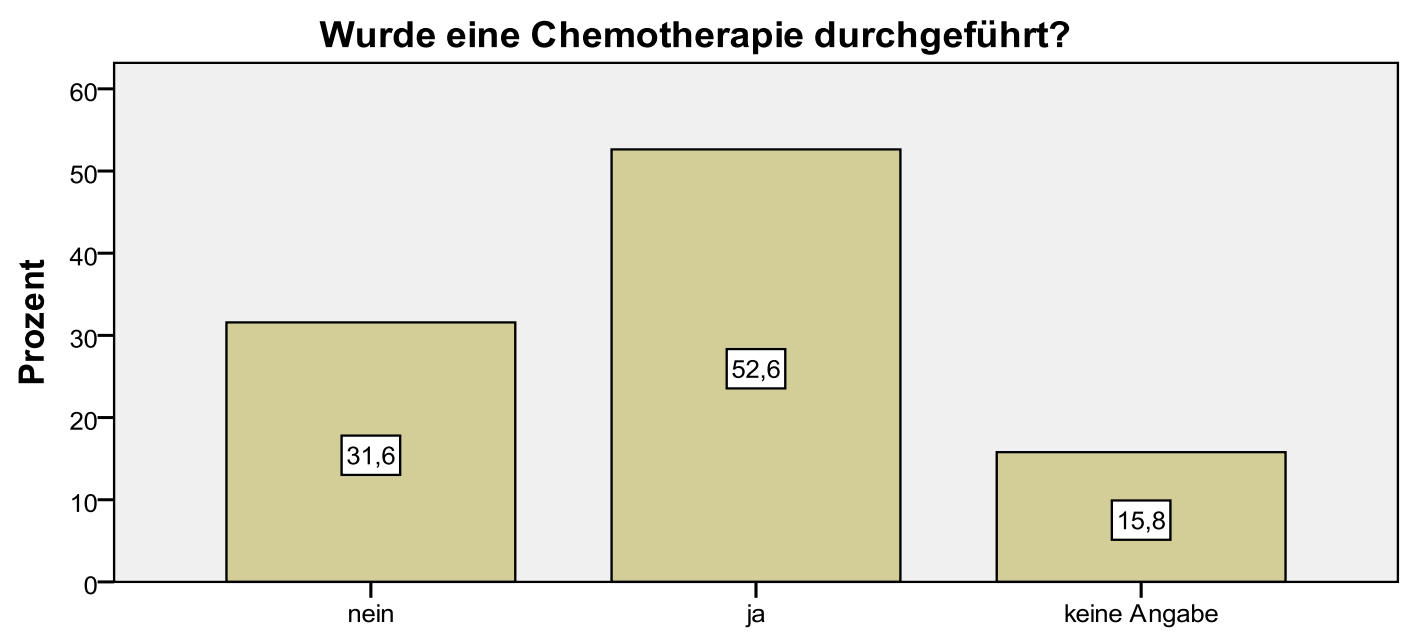

Abbildung 21: Durchführung einer Chemotherapie in der Trainingsgruppe. 
Bei 50\% der Kontrollgruppe wurde ebenfalls eine Chemotherapie durchgeführt, bei $38 \%$ wurde keine Chemotherapie appleziert. 12\% machten hierzu keine Angaben (siehe Tabelle 22 und Abb.22).

\begin{tabular}{|l|l|r|r|}
\hline \multicolumn{2}{|c|}{} & \multicolumn{1}{|c|}{ Häufigkeit } & \multicolumn{1}{c|}{ Prozent } \\
\hline \multirow{3}{*}{ Gültig } & nein & 19 & 38,0 \\
\cline { 2 - 4 } & ja & 25 & 50,0 \\
\cline { 2 - 4 } & keine Angabe & 6 & 12,0 \\
\hline
\end{tabular}

Tabelle 22: Durchführung einer Chemotherapie in der Kontrollgruppe.

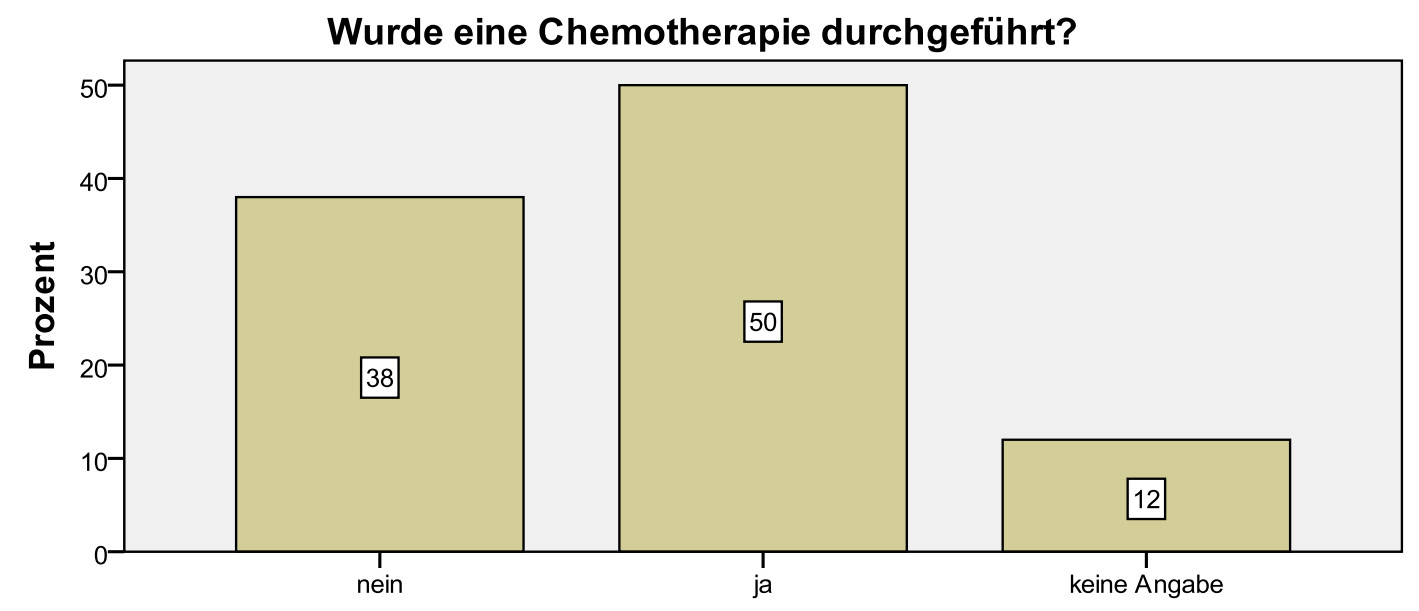

Abbildung 22: Durchführung einer Chemotherapie in der Kontrollgruppe. 
In der Trainingsgruppe gaben $43,9 \%$ der Patientinnen an, dass eine Hormontherapie durchgeführt wurde, bei 42,1\% war dies nicht der Fall. 14\% machten hierzu keine Angaben (siehe Tabelle 23 und Abb.23).

\begin{tabular}{|l|l|r|r|}
\hline \multicolumn{2}{|c|}{} & \multicolumn{1}{|c|}{ Häufigkeit } & \multicolumn{1}{c|}{ Prozent } \\
\hline \multirow{3}{*}{ Gültig } & nein & 24 & 42,1 \\
\cline { 2 - 4 } & ja & 25 & 43,9 \\
\cline { 2 - 4 } & keine Angabe & 8 & 14,0 \\
\cline { 2 - 4 } & Gesamt & 57 & 100,0 \\
\hline
\end{tabular}

Tabelle 23: Durchführung einer Hormontherapie in der Trainingsgruppe.

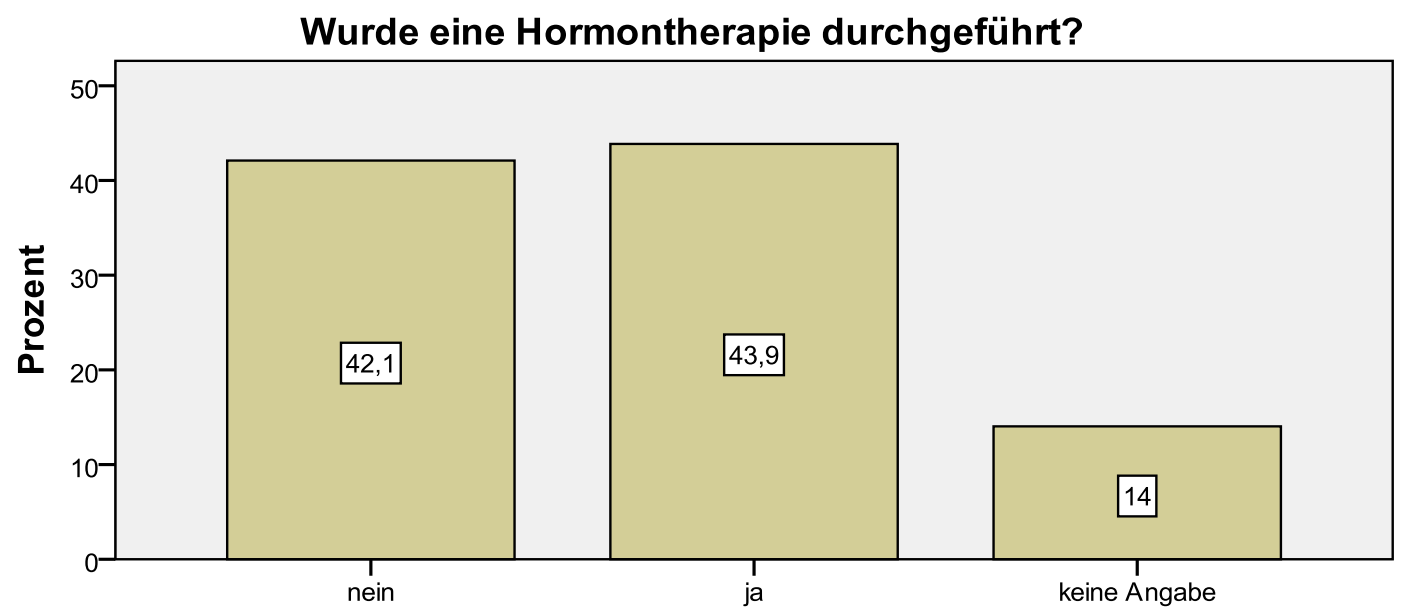

Abbildung 23: Durchführung einer Hormontherapie in der Trainingsgruppe. 
Die Frage nach einer durchgeführten Hormontherapie beantworteten $54 \%$ der Kontrollgruppe mit ja, 34\% antworteten mit nein. Keine Angaben zu dieser Frage machten 12\% der gefragten Patientinnen (siehe Tabelle 24 und Abb. 24).

\begin{tabular}{|l|l|r|r|}
\hline \multicolumn{2}{|c|}{} & Häufigkeit & \multicolumn{1}{c|}{ Prozent } \\
\hline \multirow{3}{*}{ Gültig } & nein & 17 & 34,0 \\
\cline { 2 - 4 } & ja & 27 & 54,0 \\
\cline { 2 - 4 } & keine Angabe & 6 & 12,0 \\
\cline { 2 - 4 } & Gesamt & 50 & 100,0 \\
\hline
\end{tabular}

Tabelle 24: Durchführung einer Hormontherapie in der Kontrollgruppe.

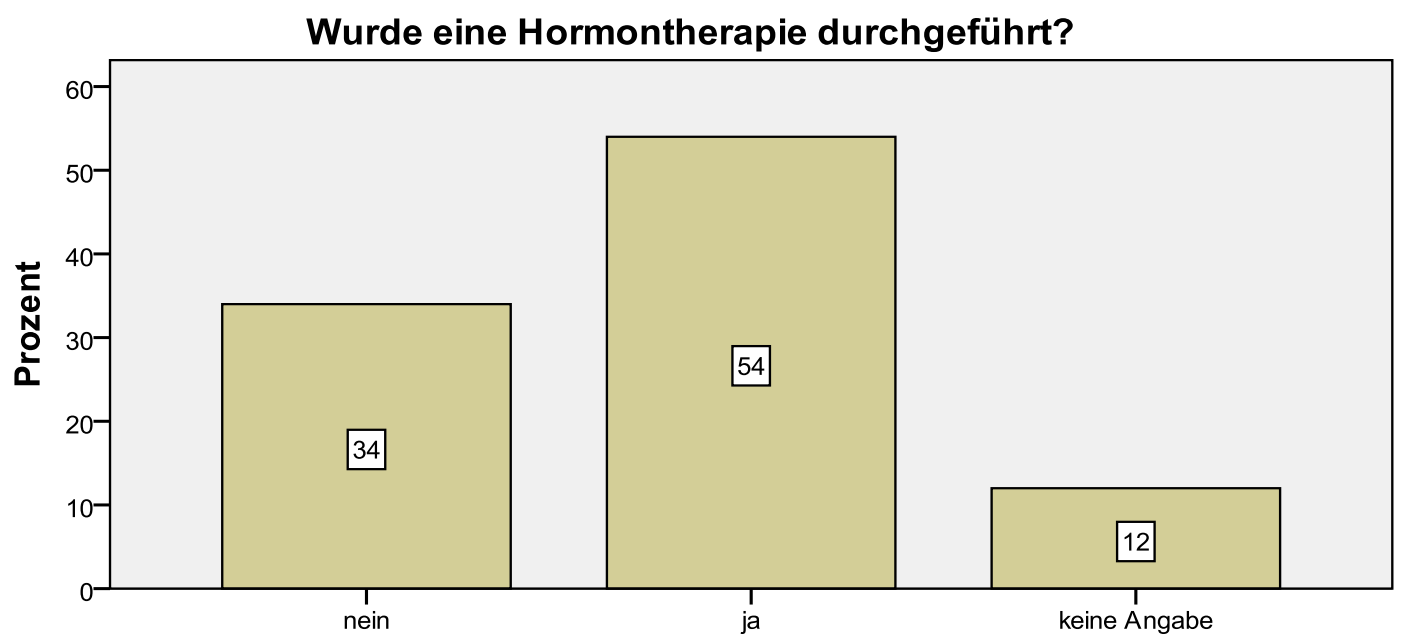

Abbildung 24: Durchführung einer Hormontherapie in der Kontrollgruppe. 
Eine Strahlentherapie wurde, wie in Tabelle 25 und Abb.25 zu sehen ist, bei 63,2\% der Patientinnen der Trainingsgruppe durchgeführt. 21,1\% der Patientinnen gaben an, dass keine Strahlentherapie durchgeführt wurde. Weitere 15,8\% machten keine Angaben zu einer durchgeführten Strahlentherapie.

\begin{tabular}{|l|l|r|r|}
\hline \multicolumn{2}{|c|}{} & Häufigkeit & \multicolumn{1}{c|}{ Prozent } \\
\hline \multirow{4}{*}{ Gültig } & nein & 12 & 21,1 \\
\cline { 2 - 4 } & ja & 36 & 63,2 \\
\cline { 2 - 4 } & keine Angabe & 9 & 15,8 \\
\cline { 2 - 4 } & Gesamt & 57 & 100,0 \\
\hline
\end{tabular}

Tabelle 25: Durchführung einer Strahlentherapie in der Trainingsgruppe.

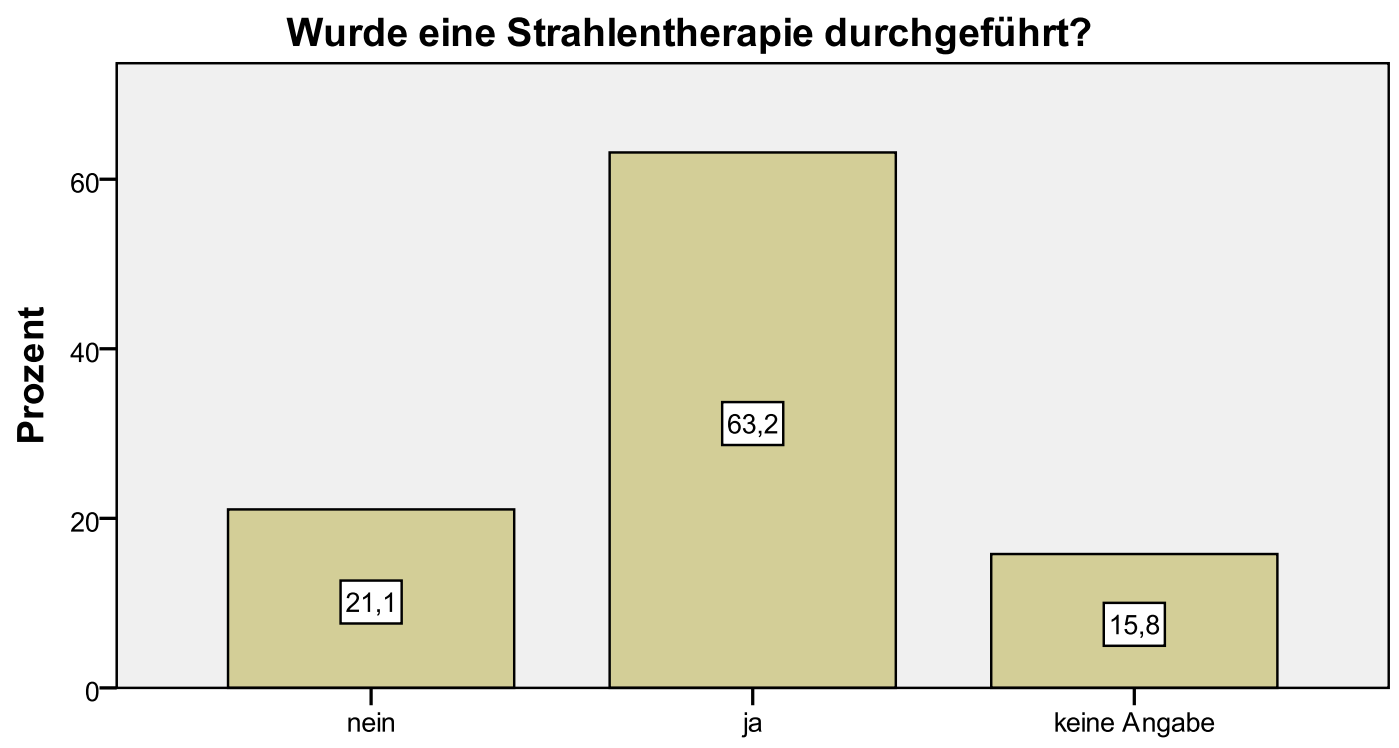

Abbildung 25: Durchführung einer Strahlentherapie in der Trainingsgruppe. 
In der Kontrollgruppe machten $66 \%$ der Patientinnen die Angabe, dass eine Strahlentherapie durchgeführt wurde, 24\% antworteten hier mit nein. 10\% machten keine Angaben (siehe Tabelle 26 und Abb. 26).

\begin{tabular}{|l|l|r|r|}
\hline \multicolumn{2}{|c|}{} & Häufigkeit & Prozent \\
\hline \multirow{3}{*}{ Gültig } & nein & 12 & 24,0 \\
\cline { 2 - 4 } & ja & 33 & 66,0 \\
\cline { 2 - 4 } & keine Angabe & 5 & 10,0 \\
\cline { 2 - 4 } & Gesamt & 50 & 100,0 \\
\hline
\end{tabular}

Tabelle 26: Durchführung einer Strahlentherapie in der Kontrollgruppe.

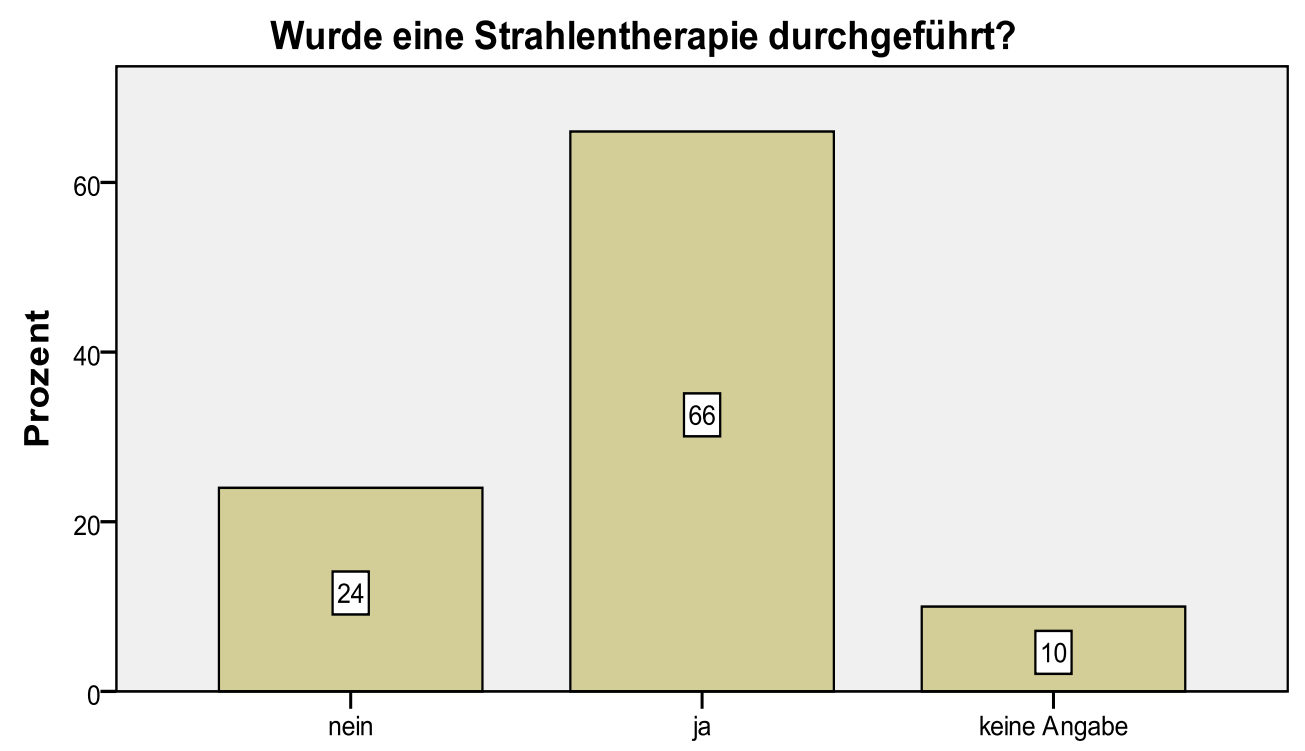

Abbildung 26: Durchführung einer Strahlentherapie in der Kontrollgruppe. 


\subsubsection{Physiotherapeutische Fragestellungen}

Abbildung 27a und b zeigen, dass $67,2 \%$ der Patientinnen der Trainingsgruppe angaben, dass die Operationsnarben zum Messzeitpunkt T1 nicht schmerzhaft sind, 32,8\% gaben Schmerzen im Operationsgebiet an.

In der Kontrollgruppe waren zum Zeitpunkt der ersten Befragung bei 58,3\% keine Schmerzen im Operationsgebiet vorhanden, $41,7 \%$ der Patientinnen gaben keine Schmerzen an.
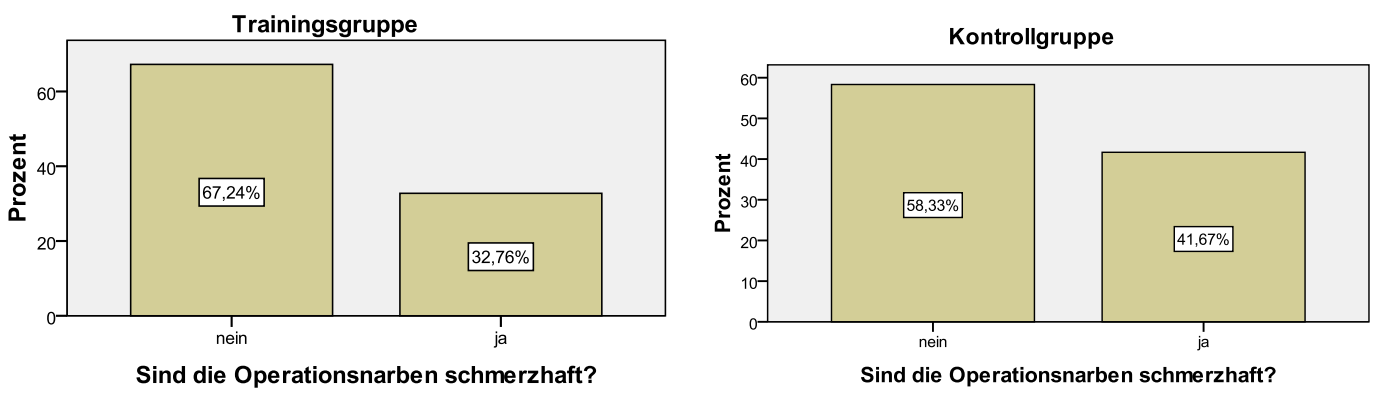

Abbildung 27 a und b: Schmerzhafte Operationsnarben.

Bei der Frage nach Schwellungen im Operationsbereich gaben 62,1\% der Patientinnen der Trainingsgruppe an, dass zum Messzeitpunkt T1 keine Schwellungen vorliegen. Bei $36,2 \%$ waren Schwellungen vorhanden, 1,7\% machten hierzu keine Angaben.

$76 \%$ der Patientinnen der Kontrollgruppe gaben an, keine Schwellungen im Operationsbereich zu haben, bei 24\% der Patientinnen lagen Schwellungen vor (siehe Abb. 28a und b).
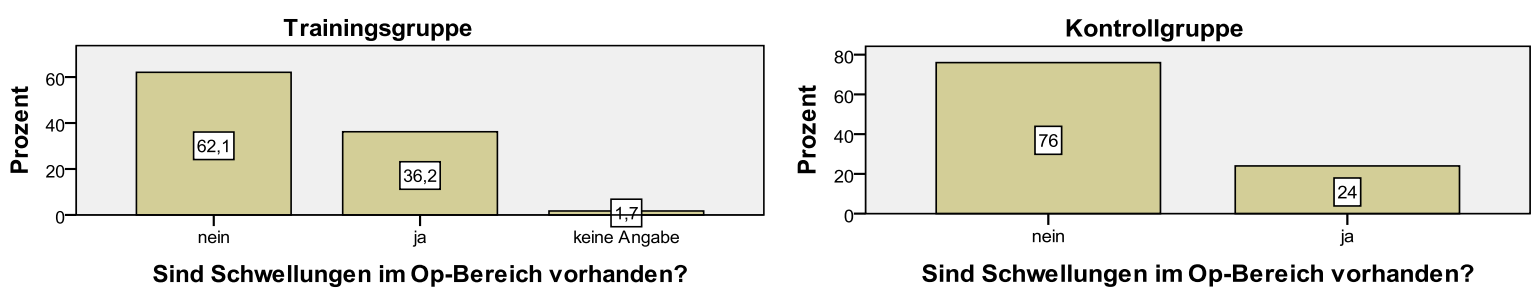

Abbildung 28 a und b: Schwellungen im Operationsbereich. 
Über schmerzhafte Muskelverspannungen im Schulter-Nacken-Bereich klagten, wie in Abb. 29a und b dargestellt, in der Trainingsgruppe an T1 79,3\% der Patientinnen. Keine derartigen Verspannungen zu haben, gaben $20,7 \%$ der Patientinnen an.

In der Kontrollgruppe gaben $70 \%$ der Patientinnen an, schmerzhafte Verspannungen im Bereich der Nackenmuskulatur zu haben, bei 30\% war dies nicht der Fall.
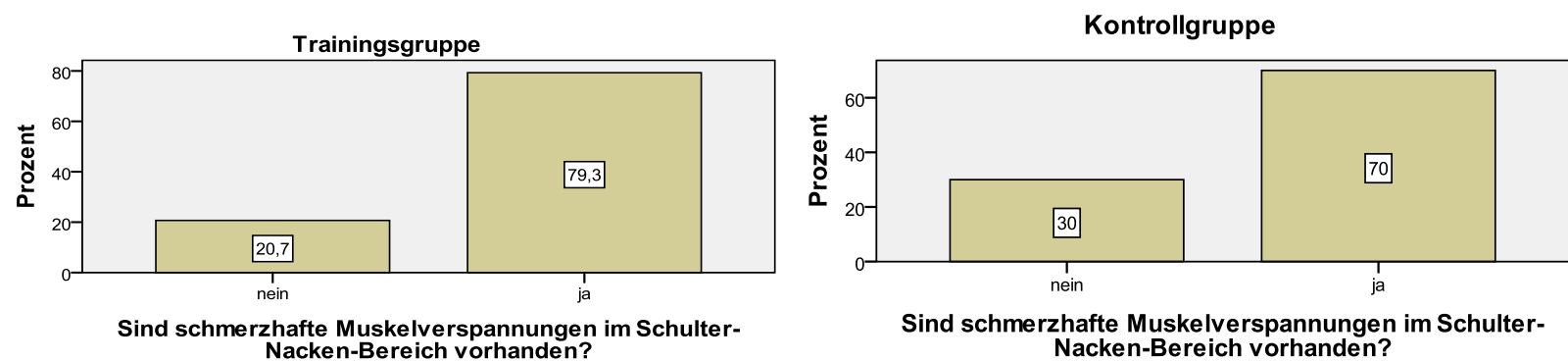

Sind schmerzhafte Muskelverspannungen im SchulterNacken-Bereich vorhanden?

Abbildung 29 a und b: Schmerzhafte Verspannungen im Schulterbereich zum Messzeitpunkt T1.

An T3 gaben 65\% der Patientinnen der Trainingsgruppe an, unter Muskelverspannungen zu leiden, 32,5\% hatten keine schmerzhaften Muskelverspannungen. 2,5\% machten hierzu keine Angaben. Von der Kontrollgruppe hatten zum Messzeitpunkt T3 73,2\% schmerzhafte Muskelverspannungen, 26,8\% hatten damit keine Probleme (siehe Abb. 30a und b).
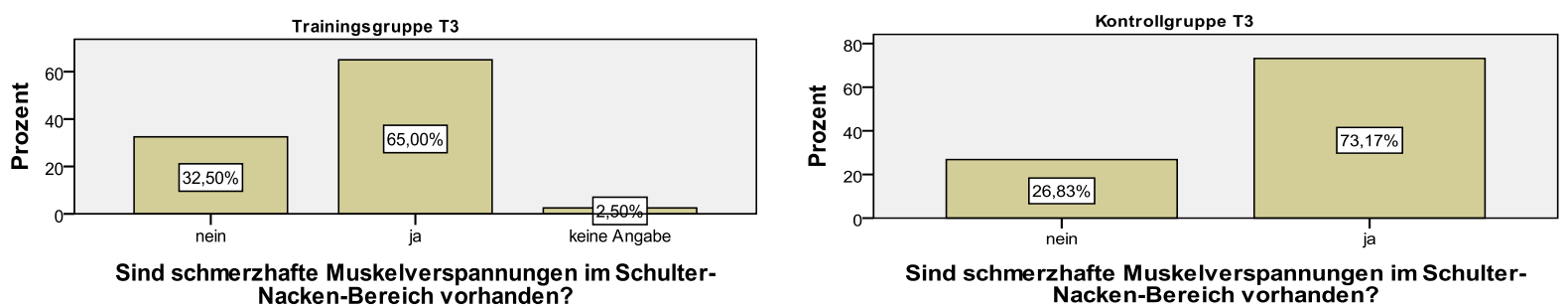

Abbildung 30 a und b: Schmerzhafte Verspannungen im Schulterbereich zum Messzeitpunkt T3 
An T4 gaben 55,9\% der Patientinnen der Trainingsgruppe schmerzhafte Muskelverspannung im Schulter-Nacken-Bereich an. 44,1\% hatten keine Verspannungen dieser Art.

In der Kontrollgruppe litten 72,5\% an T4 an schmerzhaften Muskelverspannungen, 27,5\% gaben an, keine schmerzhaften Verspannungen in der Schulter-Nacken-Region zu haben (siehe Abb. 31a und b).
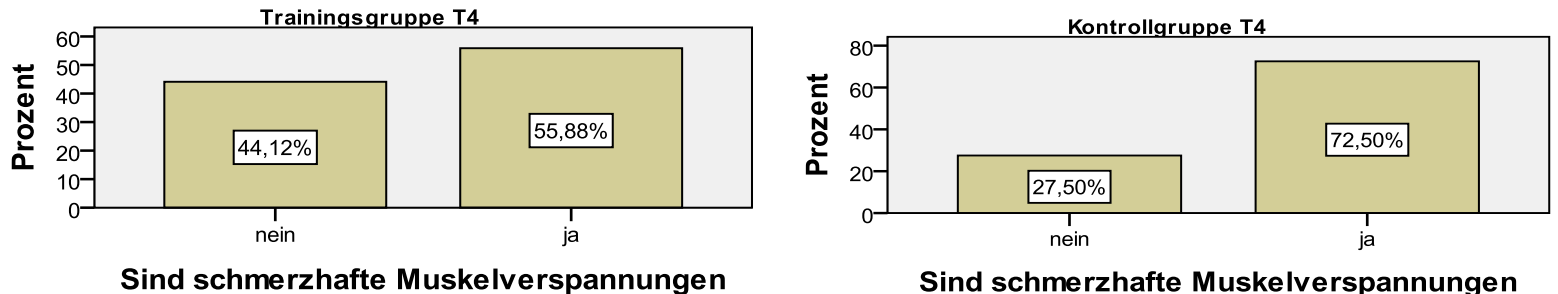

im Schulter-Nacken-Bereich vorhanden?

Sind schmerzhafte Muskelverspannungen im Schulter-Nacken-Bereich vorhanden?

Abbildung 31 a und b: Schmerzhafte Verspannungen im Schulterbereich zum Messzeitpunkt T4.

Auf die Frage nach einer erschwerten Atmung gaben $74,1 \%$ der Patientinnen der Trainingsgruppe an, keine Atemprobleme zu haben. $24,1 \%$ gaben an, dass sie unter Atembeschwerden leiden und 1,8\% machten hierzu keine Angaben.

In der Kontrollgruppe lag der Anteil von Patientinnen ohne Atembeschwerden bei 68,8\%. Unter Atembeschwerden zu leiden hatten 29,2\%, keine Angaben hierzu machten 2\% (siehe Abb. 32a und b).

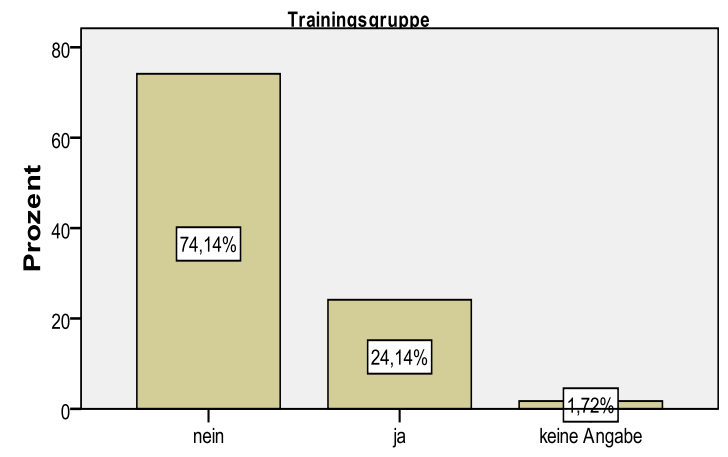

Ist die Atmung erschwert oder schmerzhaft?

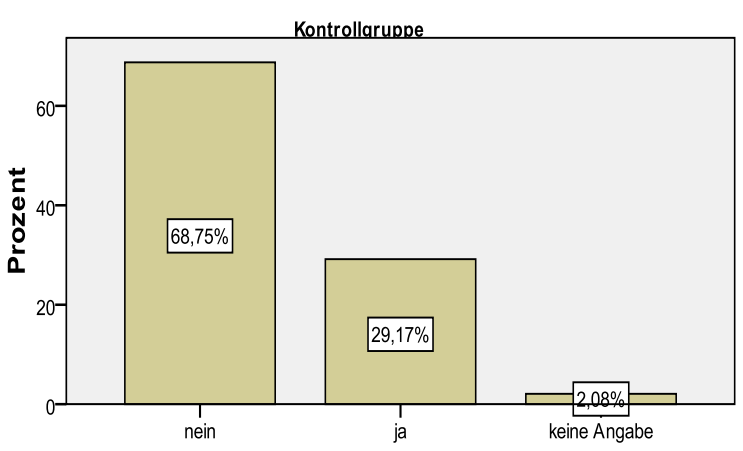

Ist die Atmung erschwert oder schmerzhaft?

Abbildung 32 a und b: Erschwerte oder schmerzhafte Atmung. 
Auf die Frage nach postoperativen Sensibilitätsstörungen in der Hand gaben 62,1\% der Patientinnen der Trainingsgruppe an, nicht von diesen betroffen zu sein. Bei 37,9\% lag eine Sensibilitätsstörung im Bereich der Hand vor.

In der Kontrollgruppe hatten 68\% keine Probleme mit Sensibilitätsstörungen in der Hand, $28 \%$ hatten Sensibilitätsstörungen in der Hand. $4 \%$ machten hier keine Angaben (siehe Abb. 33a und b).
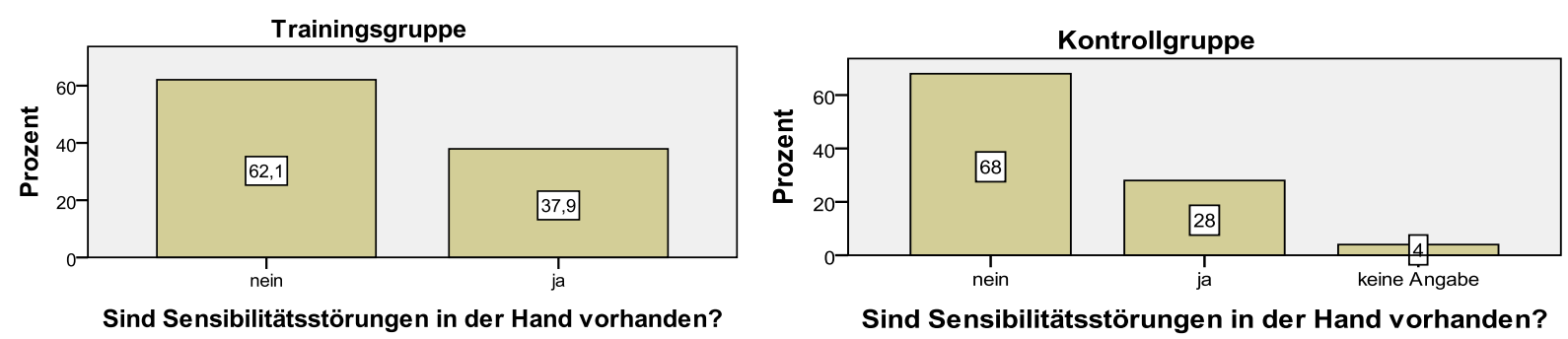

Abbildung 33 a und b: Sensibilitätsstörungen der Hand.

Am Messzeitpunkt T1 gaben 62,1\% der Patientinnen aus der Trainingsgruppe einen gefühlten Kraftverlust der operierten Seite an. 37,9\% der Patientinnen verspürten keinen Kraftverlust.

In der Kontrollgruppe war zum Messzeitpunkt T1 bei 70,8\% ein Kraftverlust auf der operierten Seite vorhanden, 27,1\% hatten keinen gefühlten Kraftverlust und 2,1\% machten hierzu keine Angaben (siehe Abb.34a und b).

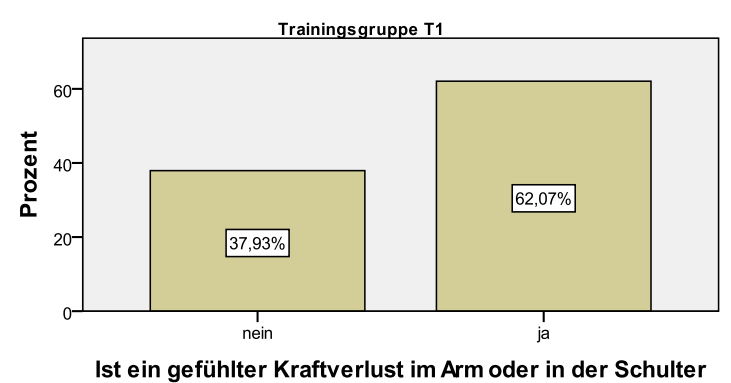
vorhanden?

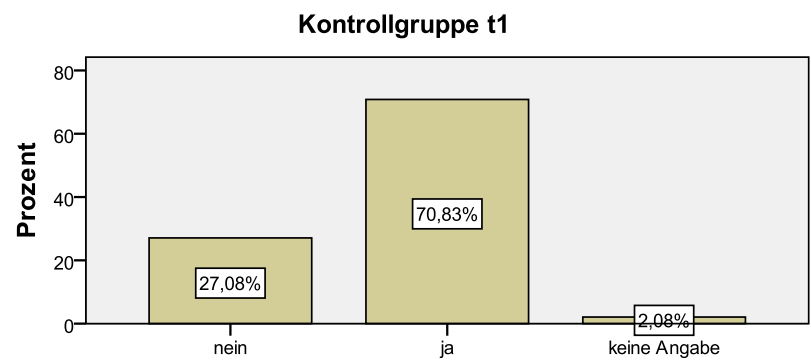

Ist ein gefühlter Kraftverlust im Arm oder in der Schulter vorhanden?

Abbildung 34 a und b: Gefühlter Kraftverlust in Arm oder Schulter zum Messzeitpunkt T1. 
Zum Messzeitpunkt T3, also 3 Monaten nach Verlassen der Rehabilitationsklinik, gaben nur noch 52,5\% der Patientinnen der Trainingsgruppe einen Kraftverlust an, 47,5\% hatten keinen Kraftverlust empfunden.

Die Patientinnen der Kontrollgruppe gaben zum Messzeitpunkt T3 zu 61\% einen gefühlten Kraftverlust an, 39\% empfanden keinen Kraftverlust der operierten Seite (siehe Abb.35a und $b$ ).
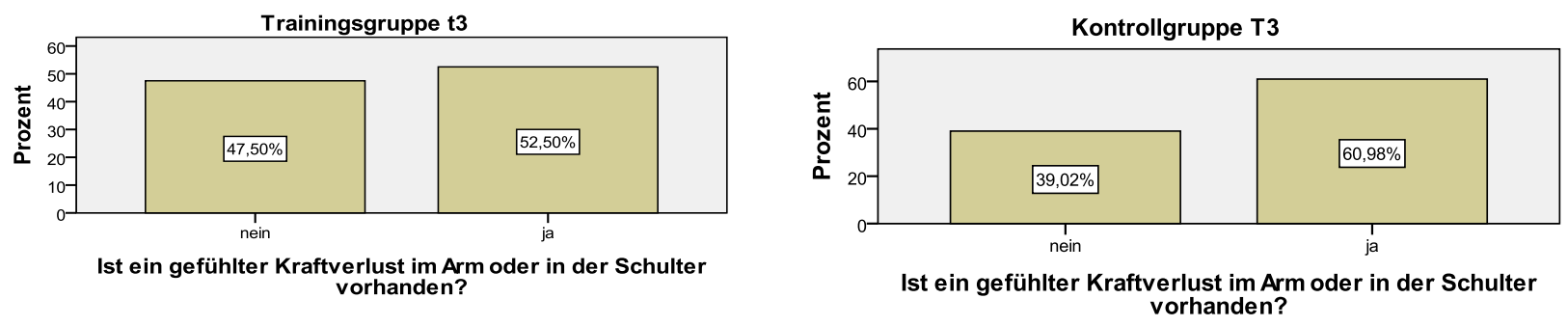

Abbildung 35 a und b: Gefühlter Kraftverlust in Arm oder Schulter zum Messzeitpunkt T3.

Sechs Monate postrehabilitativ gaben 53\% der Patientinnen der Trainingsgruppe einen gefühlten Kraftverlust der operierten Seite an, 47\% empfanden keinen merklichen Kraftverlust.

Ähnliche Werte ergaben sich bei den Patientinnen der Kontrollgruppe. 52,5\% fühlten einen Kraftverlust nach sechs Monaten, 47,5\% gaben an, keinen Kraftverlust zu verspüren (siehe Abb.36 a und b).

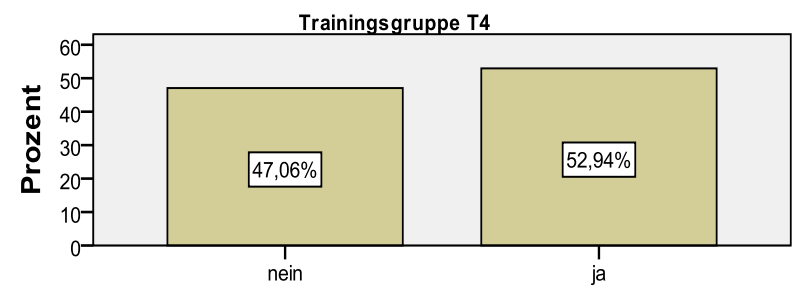

Ist ein gefühlter Kraftverlust im Arm oder in der Schulter vorhanden?

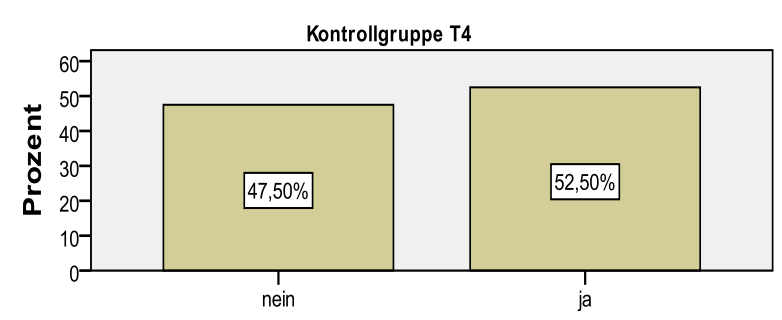

Ist ein gefühlter Kraftverlust im Arm oder in der Schulter vorhanden?

Abbildung 36 a und b: Gefühlter Kraftverlust in Arm oder Schulter zum Messzeitpunkt T4. 
Wie auf den Abbildungen 37a und b zu sehen ist, antworteten auf die Frage nach Bewegungseinschränkungen zum Messzeitpunkt T1 63,8\% der Patientinnen der Trainingsgruppe mit ja, 36,2\% gaben an, keine Bewegungseinschränkungen zu haben. Aus der Kontrollgruppe gaben 60,4\% Bewegungseinschränkungen zum Messzeitpunkt T1 an, 37,5\% hatten keine Bewegungseinschränkungen, 2,1\% machten keine Angaben.
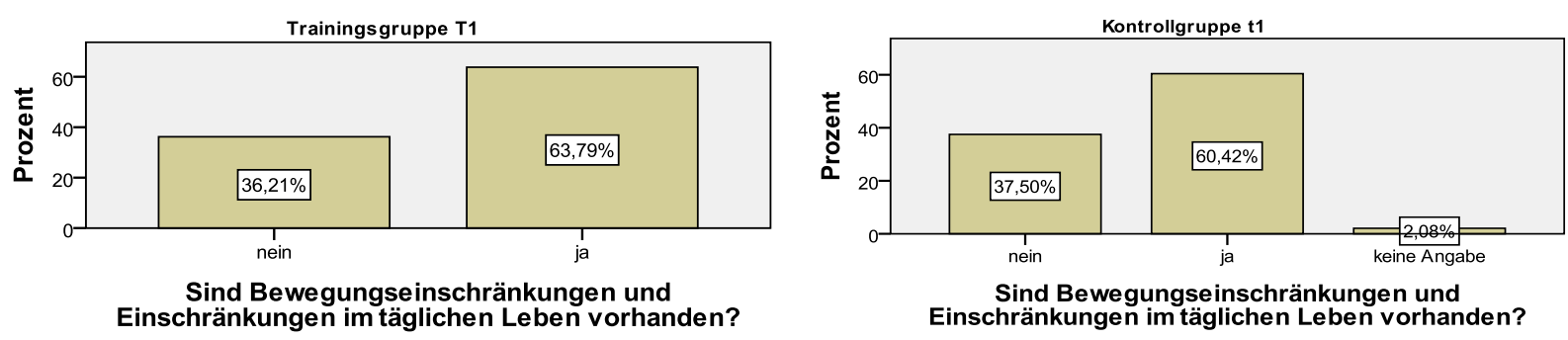

Abbildung 37 a und b: Einschränkungen im täglichen Leben zum Messzeitpunkt T1.

Am Messzeitpunkt T3 gaben 50\% der befragen Patientinnen der Trainingsgruppe Bewegungseinschränkungen und Einschränkungen im täglichen Leben an, 50\% der Patientinnen hatten hier keine Bewegungseinschränkungen.

In der Kontrollgruppe hatten an T3 noch 56,1\% Bewegungseinschränkungen, 43,9\% gaben an, keine Einschränkungen mehr zu haben (siehe Abb.38a und b).

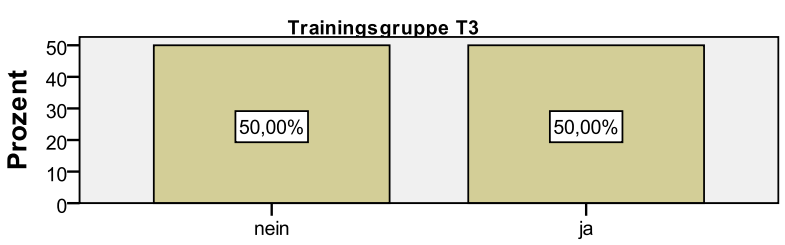

Sind Bewegungseinschränkungen und Einschränkungen im täglichen Leben vorhanden?

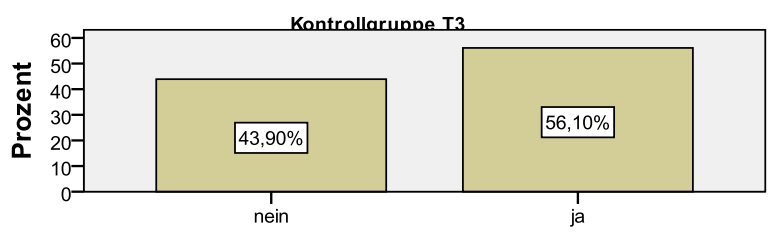

Sind Bewegungse inschränkungen und Einschränkungen im täglichen Leben vorhanden?

Abbildung 38 a und b: Einschränkungen im täglichen Leben zum Messzeitpunkt T3. 
An T4 hatten 47,1\% der Trainingsgruppenpatientinnen noch Bewegungseinschränkungen, $52,9 \%$ waren hier einschränkungsfrei.

Zum Messzeitpunkt T4 gaben 72,5\% aller Patientinnen der Kontrollgruppe an, unter Bewegungseinschränkungen oder Einschränkungen im täglichen Leben zu leiden, 27,5\% hatten keine der Beschwerden (siehe Abb. 39a und b).

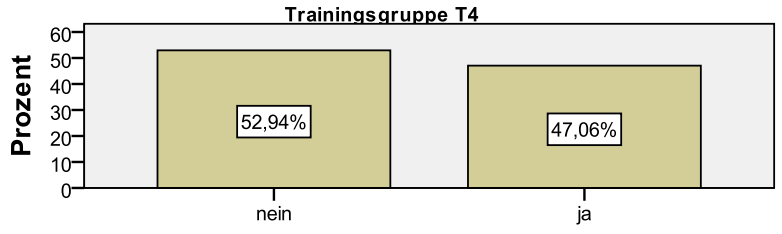

Sind Bewegungseinschränkungen und Einschränkungen im täglichen Leben vorhanden?

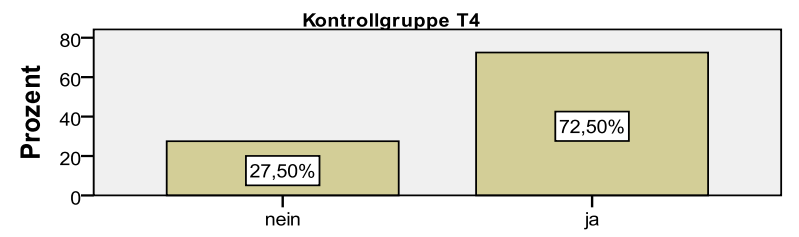

Sind Bewegungseinschränkungen und Einschränkungen im täglichen Leben vorhanden?

Abbildung 39 a und b: Einschränkungen im täglichen Leben zum Messzeitpunkt T4.

\subsubsection{Sporttherapeutische Fragebogenauswertung}

$50 \%$ der Patientinnen der Kontrollgruppe betrieben vor der Diagnose der Erkrankung regelmäßig Sport, 45,8\% nur unregelmäßig und 4,2\% gaben an, keine sportliche Aktivität ausgeübt zu haben.

In der Trainingsgruppe gaben 47,5\% der Patientinnen an, regelmäßig Sport betrieben zu haben, unregelmäßiger sportlicher Aktivität gingen 32,2\% aller Patientinnen nach. Keinen Sport betrieben 5,1\% und 15,2\% machten hierzu keine Angaben (siehe Abb.40a und b).
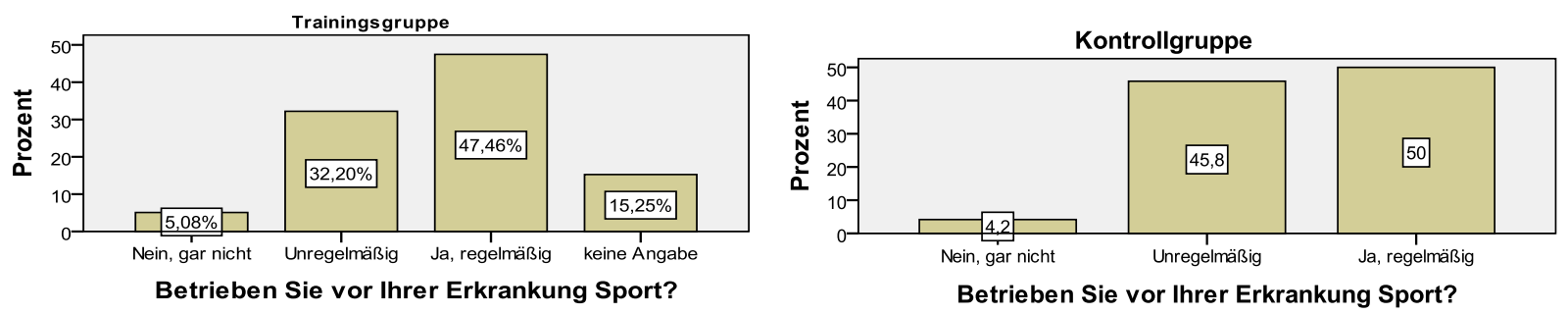

Abbildung 40 a und b: Sport vor der Erkrankung. 
Auf die Frage, welche Sportart die Patientinnen der Kontrollgruppe durchgeführt haben, gaben $47,9 \%$ an, eine Ausdauersportart durchgeführt zu haben. 16,7\% nahmen an einem Fitnesstraining oder Kurs teil, 4,2\% übten eine Spielsportart aus. Sonstige Sportarten wurden von 18,8\% ausgeübt, 12,5\% machten hierzu keine Angaben.

Eine Ausdauersportart führten 43,3\% der Patientinnen der Trainingsgruppe durch, 13,3\% besuchte ein Fitnesstraining oder Kurs. 6,7\% betrieben eine Spielsportart, 8,3\% führten sonstige Sportarten aus. 28,3\% machten hierzu keine Angaben (siehe Abb.41).

\begin{tabular}{|l|l|l|}
\hline Sportarten & Trainingsgruppe & Kontrollgruppe \\
\hline Ausdauersportarten & $43,3 \%$ & $47,9 \%$ \\
\hline Fitnesstraining & $13,3 \%$ & $16,7 \%$ \\
\hline Spielsportarten & $6,7 \%$ & $4,2 \%$ \\
\hline Sonstige Sportarten & $8,3 \%$ & $18,8 \%$ \\
\hline Keine Angabe & $28,3 \%$ & $12,5 \%$ \\
\hline
\end{tabular}

Abbildung 41: Sportarten beider Gruppen.

Zum Messzeitpunkt T1 schätzen 41,4\% der Trainingsgruppe ihre körperliche Leistungsfähigkeit als mittelmäßig ein. In der Kontrollgruppe waren es 48\%. 10,3\% der Trainingsgruppe gaben eine gute körperliche Leistungsfähigkeit an, 1,7\% eine sehr gute. Aus der Kontrollgruppe schätzten $8 \%$ ihre Leistungsfähigkeit gut ein, sehr gut gaben $0 \%$ an.

$31 \%$ der Trainingsgruppe schätzten an T1 ihrer körperlichen Leistungsfähigkeit als weniger gut ein, 15,5\% empfanden ihre Leistungsfähigkeit als schlecht. In der Kontrollgruppe gaben $28 \%$ eine weniger gute körperliche Leistungsfähigkeit an, $16 \%$ gaben diese als schlecht an (siehe Abb. 42a und b und Tabelle 27).
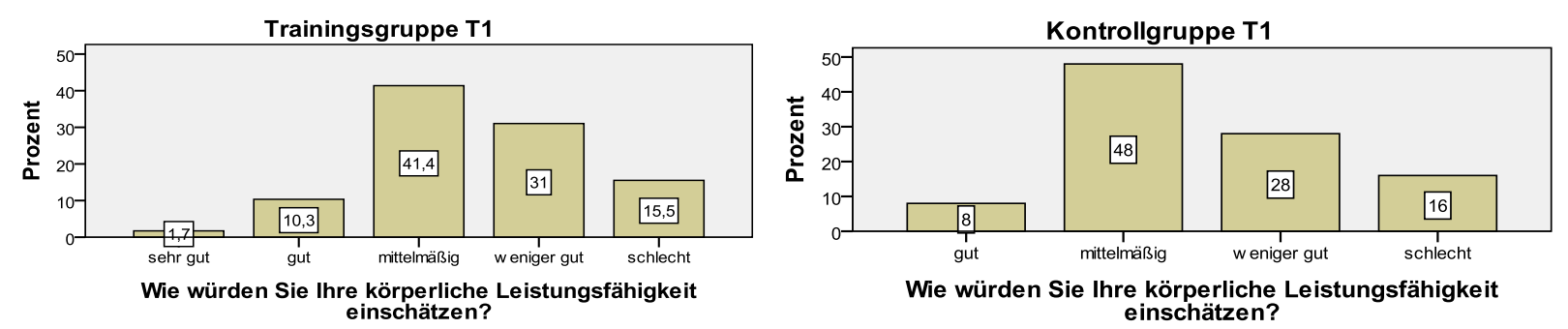

Abbildung 42 a und b: Einschätzung körperlicher Leistungsfähigkeit zum Messzeitpunkt T1. 


\begin{tabular}{|l|l|r|r|}
\hline \multicolumn{2}{|c|}{} & \multicolumn{1}{|c|}{ Häufigkeit } & Prozent \\
\hline \multirow{6}{*}{ Gültig } & 1 & 1,7 \\
\cline { 2 - 4 } & sehr gut & 6 & 10,3 \\
\cline { 2 - 4 } & gut & 24 & 41,4 \\
\cline { 2 - 4 } & mittelmäßig & 18 & 31,0 \\
\cline { 2 - 4 } & weniger gut & 9 & 15,5 \\
\cline { 2 - 4 } & schlecht & 58 & 100,0 \\
\cline { 2 - 4 } & Gesamt & & 10 \\
\hline
\end{tabular}

Tabelle 27: Einschätzung körperlicher Leistungsfähigkeit der Trainingsgruppe zum Messzeitpunkt T1.

\begin{tabular}{|l|l|r|r|}
\hline \multicolumn{2}{|c|}{} & \multicolumn{1}{|c|}{ Häufigkeit } & \multicolumn{1}{l|}{ Prozent } \\
\hline \multirow{4}{*}{ Gültig } & gut & 4 & 8,0 \\
\cline { 2 - 4 } & mittelmäßig & 24 & 48,0 \\
\cline { 2 - 4 } & weniger gut & 14 & 28,0 \\
\cline { 2 - 4 } & schlecht & 8 & 16,0 \\
\hline
\end{tabular}

Tabelle 28: Einschätzung körperlicher Leistungsfähigkeit der Kontrollgruppe zum Messzeitpunkt T1. 
Zum Messzeitpunkt T3, wie in Abbildung 43a sowie in Tabelle 29 dargestellt, schätzten 47,5\% der Trainingsgruppe inre körperliche Leistungsfähigkeit als gut ein, 42,5\% als mittelmäßig. Aus der Kontrollgruppe kamen 19,5\% auf das Ergebnis gut, $61 \%$ fühlten sich nur mittelmäßig leistungsfähig (siehe Abb.43b und Tabelle 30).

7,5\% der Trainingsgruppe sahen ihre Leistungsfähigkeit als weniger gut an, 2,5\% als schlecht. Aus der Kontrollgruppe gaben 7,3\% eine weniger gute körperliche Leistungsfähigkeit an, ebenfalls 7,3\% empfanden diese als schlecht. Allerdings gab es $2,4 \%$ der Kontrollgruppe, die ihre körperliche Leistungsfähigkeit als sehr gut einschätzten, weitere 2,4\% machten keine Angaben.
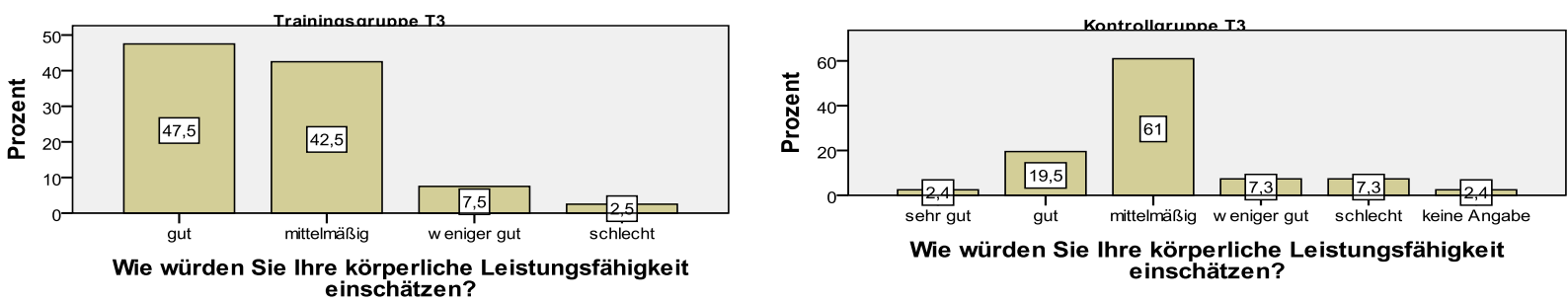

Abbildung 43 a und b: Einschätzung körperlicher Leistungsfähigkeit zum Messzeitpunkt T3.

\begin{tabular}{|l|l|r|r|}
\hline \multicolumn{2}{|c|}{} & Häufigkeit & \multicolumn{1}{c|}{ Prozent } \\
\hline \multirow{6}{*}{ Gültig } & gut & 19 & 47,5 \\
\cline { 2 - 4 } & mittelmäßig & 17 & 42,5 \\
\cline { 2 - 4 } & weniger gut & 3 & 7,5 \\
\cline { 2 - 4 } & schlecht & 1 & 2,5 \\
\cline { 2 - 4 } & Gesamt & 40 & 100,0 \\
\hline
\end{tabular}

Tabelle 29: Einschätzung körperlicher Leistungsfähigkeit der Trainingsgruppe zum Messzeitpunkt T3.

\begin{tabular}{|l|l|r|r|}
\hline \multicolumn{2}{|c|}{} & Häufigkeit & \multicolumn{1}{c|}{ Prozent } \\
\hline \multirow{6}{*}{ Gültig } & sehr gut & 1 & 2,4 \\
\cline { 2 - 4 } & gut & 8 & 19,5 \\
\cline { 2 - 4 } & mittelmäßig & 25 & 61,0 \\
\cline { 2 - 4 } & weniger gut & 3 & 7,3 \\
\cline { 2 - 4 } & schlecht & 3 & 7,3 \\
\hline
\end{tabular}

Tabelle 30: Einschätzung körperlicher Leistungsfähigkeit der Kontrollgruppe zum Messzeitpunkt T3. 
An T4 schätzten 52,9\% der Trainingsgruppe ihre körperliche Leistungsfähigkeit als gut ein, $41,2 \%$ als mittelmäßig. 5,9\% empfanden diese sogar als sehr gut.

In der Kontrollgruppe gaben $51,2 \%$ der Patientinnen an T4 an, eine mittelmäßige körperliche Leistungsfähigkeit zu haben, 19,5\% empfanden diese als weniger gut. 17,1\% schätzten ihre körperliche Leistungsfähigkeit als gut ein, 2,4\% als sehr gut. 9,8\% machten hierzu keine Angaben (siehe Abb. 44a und b und Tabelle 31 und 32).
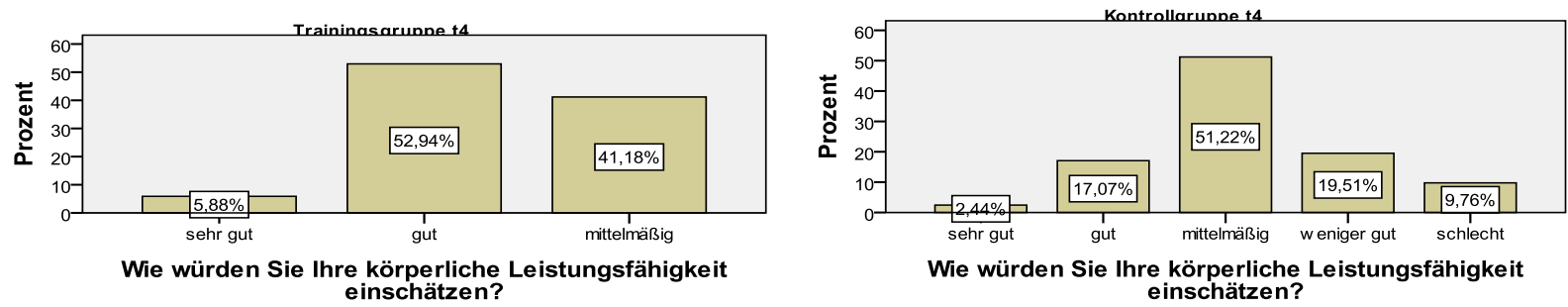

Abbildung 44 a und b: Einschätzung körperlicher Leistungsfähigkeit zum Messzeitpunkt T4.

\begin{tabular}{|l|l|r|r|}
\hline \multicolumn{2}{|c|}{} & \multicolumn{1}{|c|}{ Häufigkeit } & \multicolumn{1}{c|}{ Prozent } \\
\hline \multirow{6}{*}{ Gültig } & sehr gut & 2 & 5,9 \\
\cline { 2 - 4 } & gut & 18 & 52,9 \\
\cline { 2 - 4 } & mittelmäßig & 14 & 41,2 \\
\cline { 2 - 4 } & Gesamt & 34 & 100,0 \\
\hline
\end{tabular}

Tabelle 31: Einschätzung körperlicher Leistungsfähigkeit der Trainingsgruppe zum Messzeitpunkt T4.

\begin{tabular}{|l|l|r|r|}
\hline \multicolumn{2}{|c|}{} & Häufigkeit & \multicolumn{1}{c|}{ Prozent } \\
\hline \multirow{4}{*}{ Gültig } & sehr gut & 1 & 2,4 \\
\cline { 2 - 4 } & gut & 7 & 17,1 \\
\cline { 2 - 4 } & mittelmäßig & 21 & 51,2 \\
\cline { 2 - 4 } & weniger gut & 8 & 19,5 \\
\cline { 2 - 4 } & schlecht & 4 & 9,8 \\
\hline
\end{tabular}

Tabelle 32: Einschätzung körperlicher Leistungsfähigkeit der Kontrollgruppe zum Messzeitpunkt T4. 


\subsection{Ergebnisse des MFI}

Der allgemeine Fatigue Score des MFI, dargestellt in Abb.45 und Tabelle 33, lag in der Trainingsgruppe zum Messzeitpunkt $T 1$ bei $68,39 \pm 19,93$, in der Kontrollgruppe bei $65,18 \pm 19,72$. Zum Messzeitpunkt T2 lag die Trainingsgruppe signifikant verbessert bei einem Score von $56,25 \pm 22,41$ und die Kontrollgruppe, ebenfalls signifikant verbessert gegenüber T1 bei 56,38 $\pm 25,24$. Die Messzeitpunkte T3 und T4 zeigten in der Trainingsgruppe eine weitere signifikante Reduktion auf $45,58 \pm 20,97$ und $46,40 \pm 20,07$ respektive. In der Kontrollgruppe stieg der Score zum Messzeitpunkt T3 auf 57,34 $\pm 24,06$ um zum Messzeitpunkt T4 auf 55,45 $\pm 22,08$ zu fallen. Die Messergebnisse an T3 und T4 der Trainingsgruppe waren signifikant gegenüber T2.

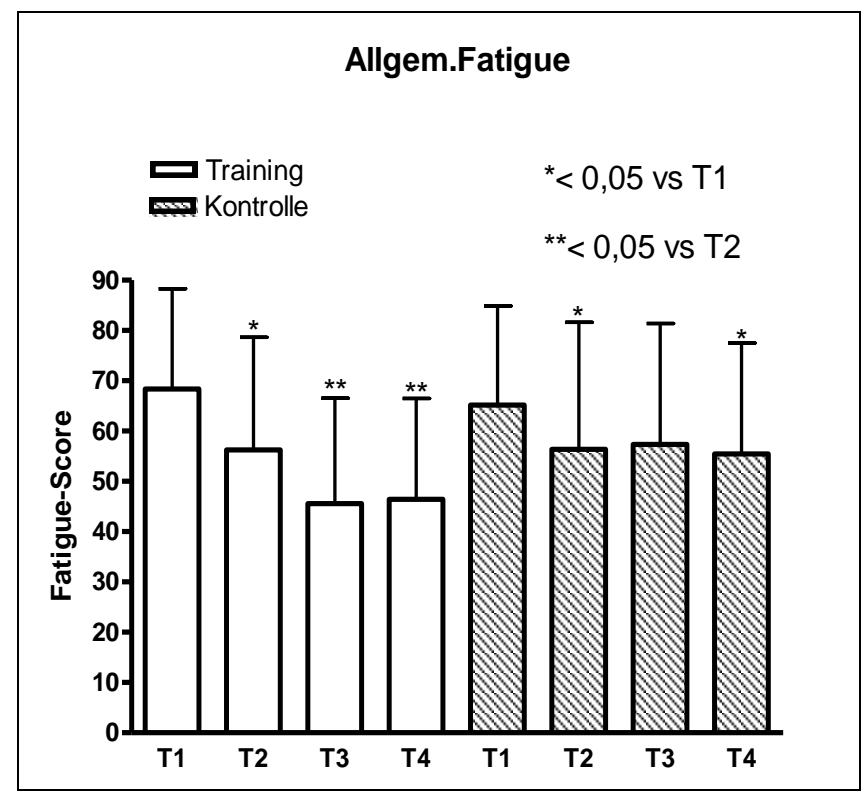

Abbildung 45: Graph der allgemeinen Fatigue des MFI für die Trainings- und Kontrollgruppe von T1T4.

\begin{tabular}{|l|l|l|l|l|l|l|l|l|}
\hline & Tr1 & Tr2 & Tr3 & Tr4 & K1 & K2 & K3 & K4 \\
\hline Mean & 68,39 & 56,25 & 45,58 & 46,4 & 65,18 & 56,38 & 57,34 & 55,45 \\
\hline SD( \pm ) & 19,93 & 22,41 & 20,97 & 20,07 & 19,72 & 25,24 & 24,06 & 22,08 \\
\hline
\end{tabular}

Tabelle 33: Allgemeiner Fatigue-Scores des MFI für die Trainingsgruppe (Tr1-4) und die Kontrollgruppe (K1-4) für die Messzeitpunkte T1-T4. 
Die Messwerte der physischen Fatigue lagen in der Kontrollgruppe zu T1 bei 56,58 $\pm 22,60$, in der Trainingsgruppe bei 56,34 $\pm 7,8$. Der Messzeitpunkt T2 ergab für die Kontrollgruppe

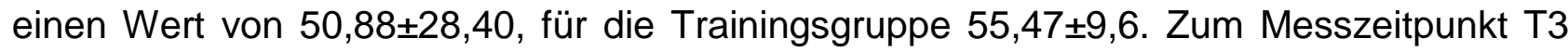

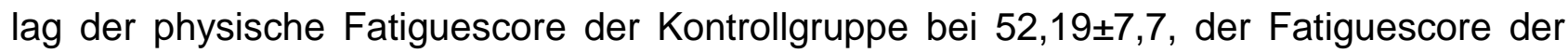

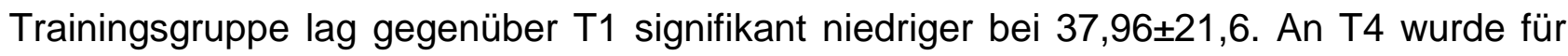
die Kontrollgruppe ein Score von 47,76 $\pm 27,2$ ermittelt, der Score der Trainingsgruppe lag erneut signifikant niedriger gegenüber $\mathrm{T} 1$ bei $33,3 \pm 19,4$ (siehe Abb. $46 \mathrm{a}$ und $\mathrm{b}$, sowie Tabelle 34).

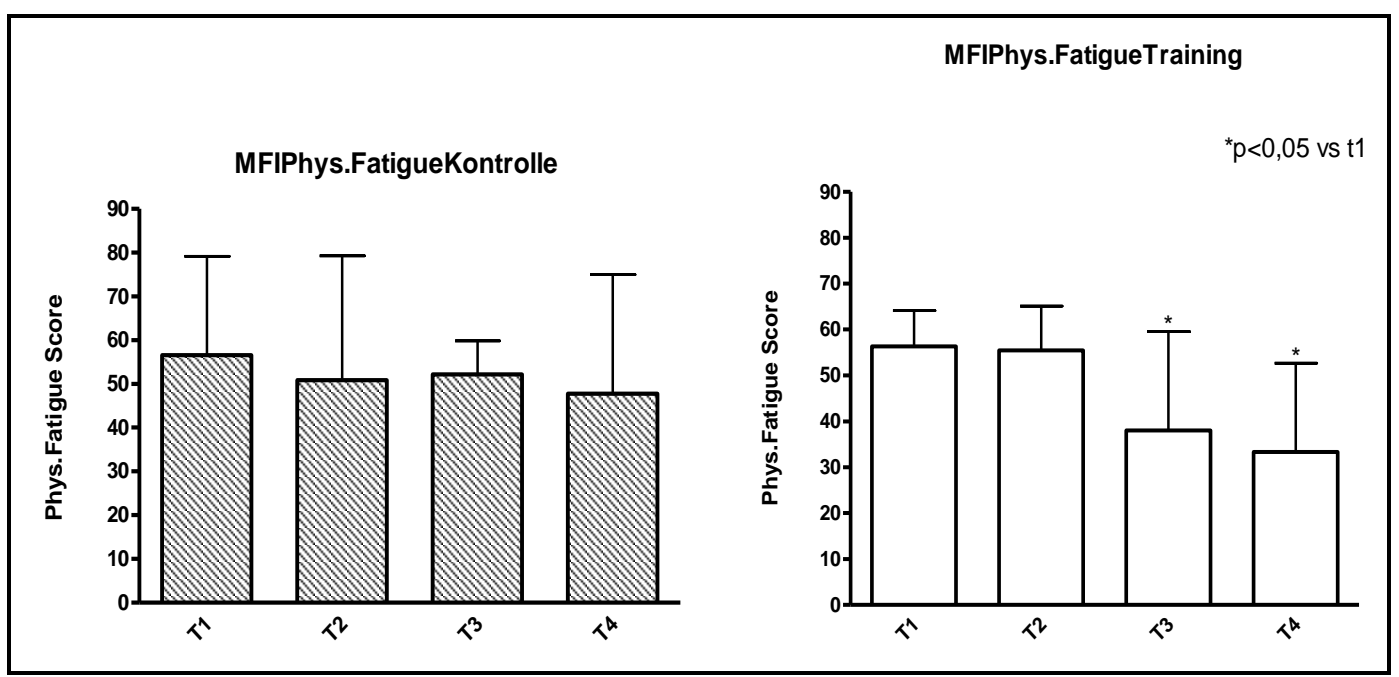

Abbildung 46 a und b: Graphen der physischen Fatigue des MFI für die Trainings- und Kontrollgruppe von T1-T4.

\begin{tabular}{|l|l|l|l|l|l|l|l|l|}
\hline & Tr1 & Tr2 & Tr3 & Tr4 & K1 & K2 & K3 & K4 \\
\hline Mean & 56,34 & 55,47 & 37,96 & 33,33 & 56,58 & 50,88 & 52,19 & 47,76 \\
\hline SD( \pm ) & 7,78 & 9,61 & 21,58 & 19,37 & 22,6 & 28,4 & 7,69 & 27,22 \\
\hline
\end{tabular}

Tabelle 34: Physischer Fatigue-Score des MFI für die Trainingsgruppe (Tr1-4) und die Kontrollgruppe (K1-4) für die Messzeitpunkte T1-T4. 
Der Ausgangswert (T1) für die mentale Fatigue der Kontrollgruppe lag bei $52,9 \pm 26,2$, in der Trainingsgruppe lag dieser Wert bei 63,93 $\pm 24,1$. Zum Messzeitpunkt T2 sank der Wert in der Kontrollgruppe auf $48 \pm 28,4$, in der Trainingsgruppe sank der Wert signifikant gegenüber T1 auf 53,91 $\pm 25,5$. T3 ergab für die Kontrollgruppe einen Score von $46,72 \pm 30$, für die Trainingsgruppe einen erneut signifikant niedrigeren Score gegenüber T1 von 42,53 $\pm 24,5$. Am Messzeitpunkt T4 war der Score in der Kontrollgruppe auf 44,23 $\pm 26,61$ gesunken, der Wert der Trainingsgruppe lag bei 37,69 $\pm 25,5$ (siehe Abb. 47a und b und Tabelle 35).

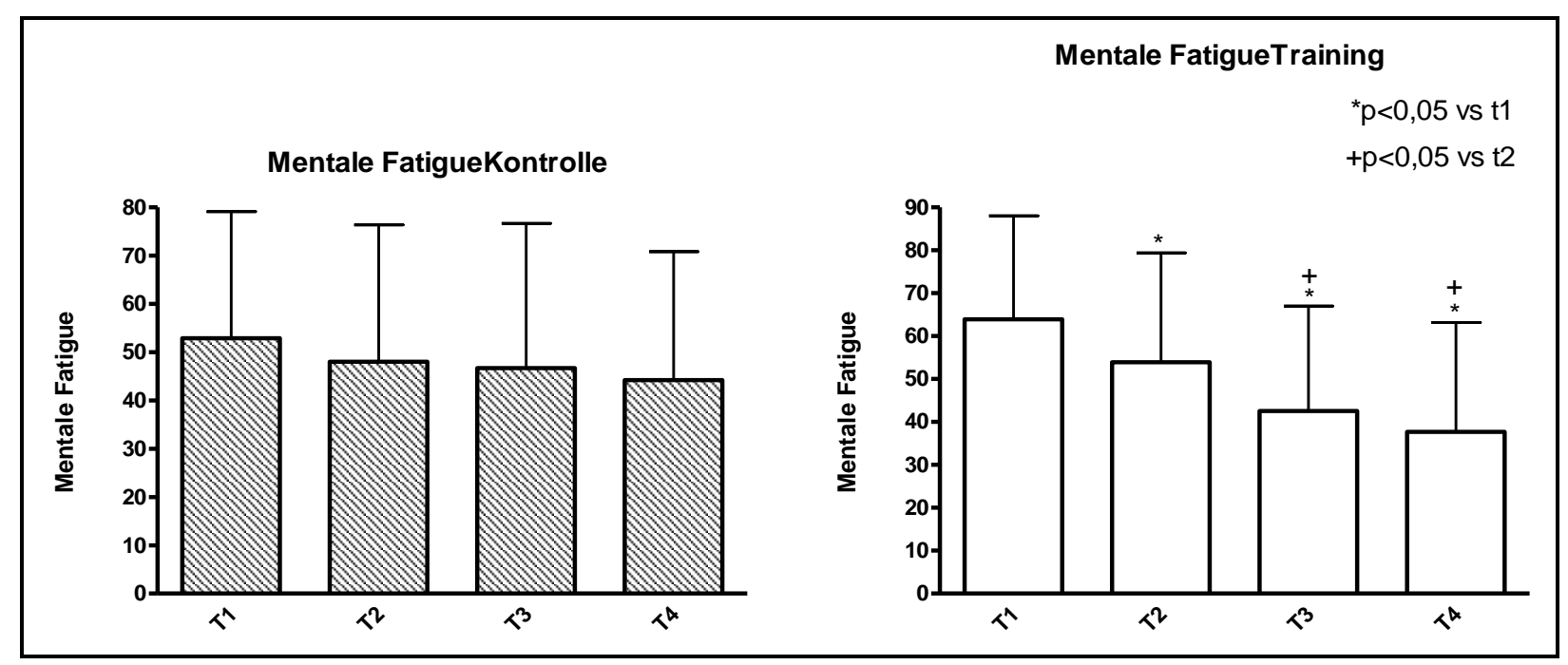

Abbildung 47 a und b: Graphen der mentalen Fatigue des MFI für die Trainings- und Kontrollgruppe von T1-T4.

\begin{tabular}{|l|l|l|l|l|l|l|l|l|}
\hline & Tr1 & Tr2 & Tr3 & Tr4 & K1 & K2 & K3 & K4 \\
\hline Mean & 63,93 & 53,91 & 42,53 & 37,69 & 52,9 & 48,0 & 46,72 & 44,23 \\
\hline SD ( $\mathbf{)}$ & 24,08 & 25,48 & 24,5 & 25,51 & 26,22 & 28,38 & 29,69 & 26,61 \\
\hline
\end{tabular}

Tabelle 35: Mentaler Fatigue-Score des MFI für die Trainingsgruppe (Tr1-4) und die Kontrollgruppe (K1-4) für die Messzeitpunkte T1-T4. 
Die Auswertung der reduzierten Aktivität ergab, dargestellt in den Abbildungen 48a und b, sowie in Tabelle 36, für die Kontrollgruppe bei T1 einen Score von 56,36 $\pm 26,4$, für die Trainingsgruppe einen Score von 58,93 $\pm 24,1$. Zu T2 lag der Wert der Kontrollgruppe bei $50,88 \pm 24,1$, der Wert der Trainingsgruppe signifikant niedriger gegenüber T1 bei $48,83 \pm 23,1$. Die Kontrollgruppe hatte an T3 einen Score von 47,97 \pm 26 , die Trainingsgruppe eine signifikant niedrigeren Wert gegenüber T1 und T2 von 34,91 \pm 23 . Zum Messzeit-

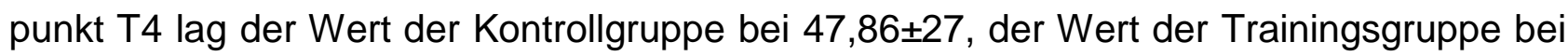
$32,01 \pm 22,1$.

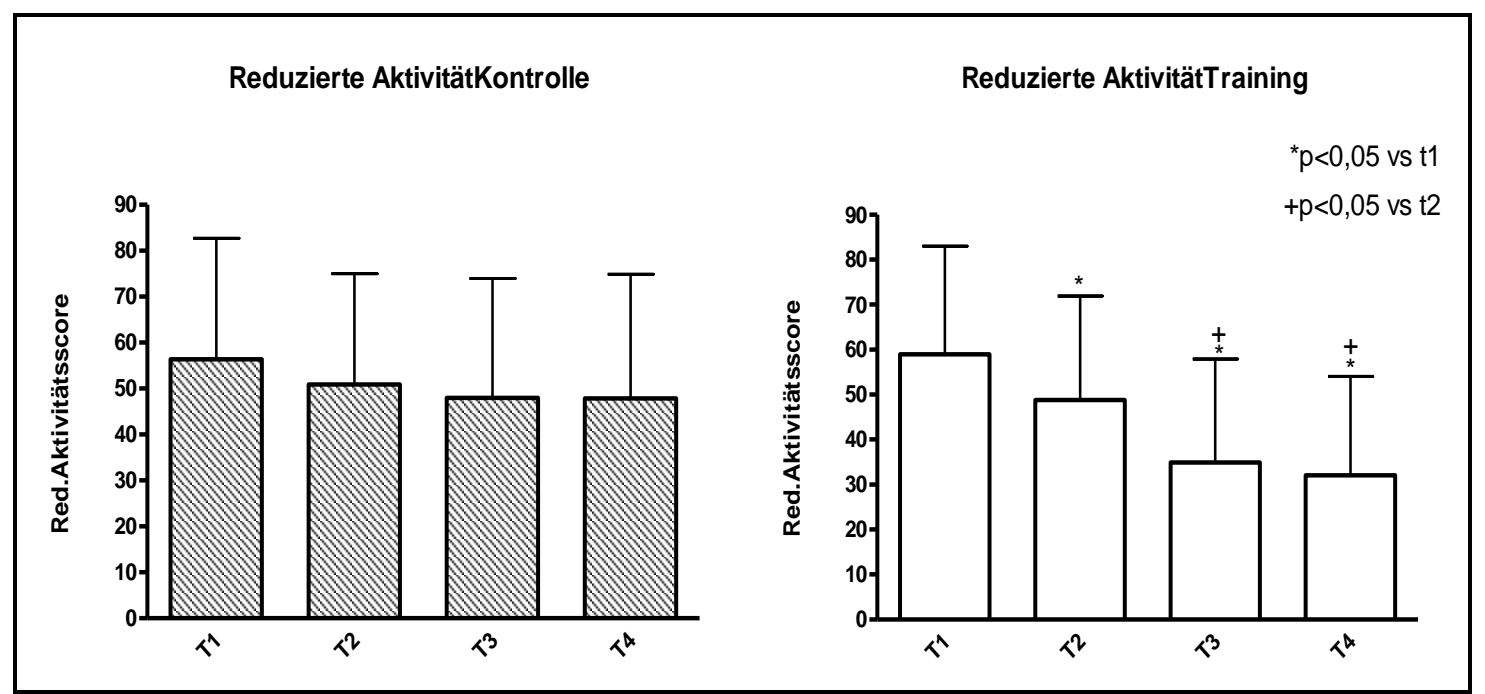

Abbildung 48 a und b: Graphen der reduzierten Aktivität des MFI für die Trainings- und Kontrollgruppe von T1-T4.

\begin{tabular}{|l|l|l|l|l|l|l|l|l|}
\hline & Tr1 & Tr2 & Tr3 & Tr4 & K1 & K2 & K3 & K4 \\
\hline Mean & 58,93 & 48,83 & 34,91 & 32,01 & 56,36 & 50,88 & 47,97 & 47,86 \\
\hline SD( \pm$)$ & 24,07 & 23,09 & 23,01 & 22,08 & 26,37 & 24,12 & 26,0 & 27,0 \\
\hline
\end{tabular}

Tabelle 36: Reduzierter Aktivität-Score des MFI für die Trainingsgruppe (Tr1-4) und die Kontrollgruppe (K1-4) für die Messzeitpunkte T1-T4. 
Der Wert der reduzierten Motivation der Kontrollgruppe (siehe Abb.49a und Tabelle 37) lag zu T1 bei 36,5 $\pm 21,2$, in der Trainingsgruppe bei 39,02 $\pm 20,7$ (siehe Abb.47b und Tabelle 37). Zum Messzeitpunkt T2 lag die Kontrollgruppe bei 32,75 $\pm 24,1$, die Trainingsgruppe bei 32,55 \pm 20 . Die Auswertung von T3 ergab für die Kontrollgruppe einen Wert der reduzierten Motivation von $33,44 \pm 21,5$, die Trainingsgruppe lag nun signifikant niedriger gegenüber T1 bei 28,2 $\pm 20,8$. Der letzte Messzeitpunkt T4 ergab für die Kontrollgruppe einen Wert von 34,13 $\pm 25,4$, der Wert der Trainingsgruppe lag erneut signifikant niedriger gegenüber $\mathrm{T} 1$ und auch $\mathrm{T} 2$ bei $21,02 \pm 18$.

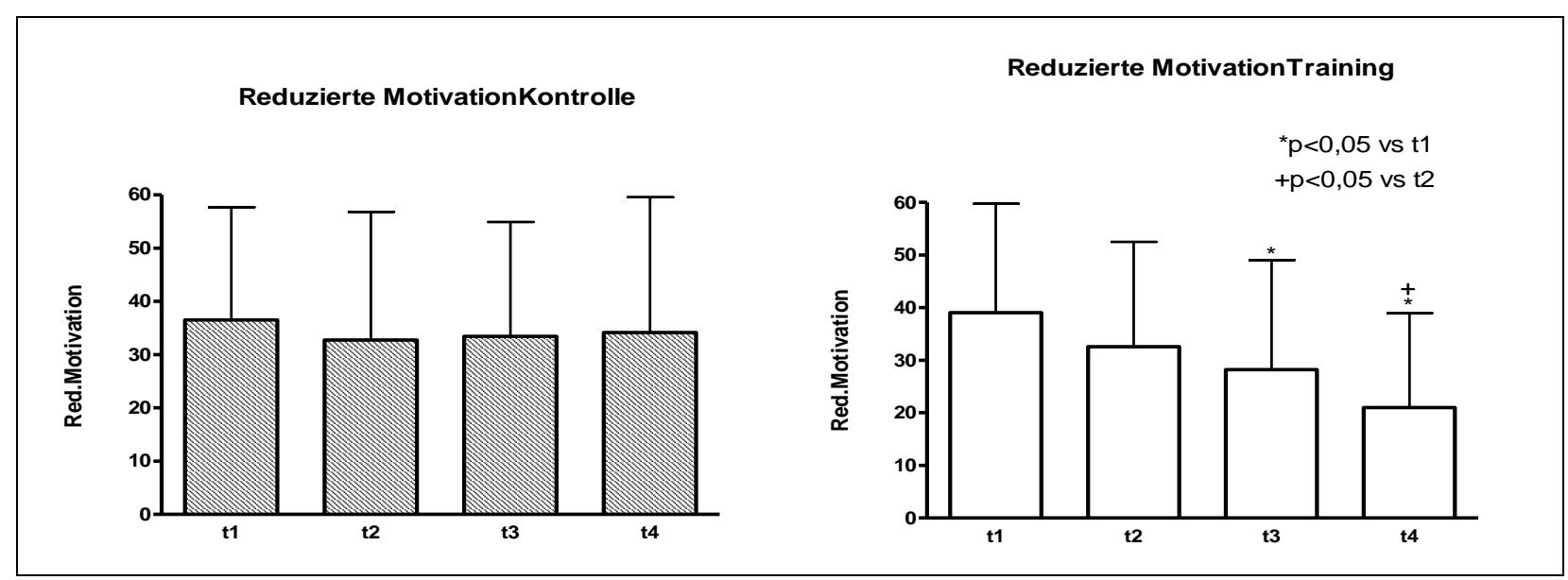

Abbildung 49 a und b: Graphen der reduzierten Motivation des MFI für die Trainings- und Kontrollgruppe von T1-T4.

\begin{tabular}{|l|l|l|l|l|l|l|l|l|}
\hline & Tr1 & Tr2 & Tr3 & Tr4 & K1 & K2 & K3 & K4 \\
\hline Mean & 39,02 & 32,55 & 28,20 & 21,02 & 36,5 & 32,75 & 33,44 & 34,13 \\
\hline SD( \pm ) & 20,73 & 19,97 & 20,83 & 17,94 & 21,15 & 24,08 & 21,49 & 25,44 \\
\hline
\end{tabular}

Tabelle 37: Reduzierter Motivation-Score des MFI für die Trainingsgruppe (Tr1-4) und die Kontrollgruppe (K1-4) für die Messzeitpunkte T1-T4. 


\subsection{Ergebnisse des EORTC QLQ C-30}

In der Trainingsgruppe lag der Score des Global Health Status an $T 1$ bei $50,42 \pm 19,84$, in

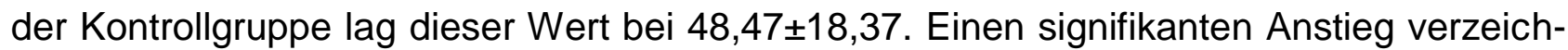
nete die Trainingsgruppe von T1 nach T2, wobei der Wert bei 60,88 $\pm 17,36$ lag. Der Global Health Status der Kontrollgruppe stieg ebenfalls auf 55,78 $\pm 23,95$ an, jedoch nicht signifikant. Zu den Messzeitpunkten T3 und T4 lagen die Werte in der Trainingsgruppe weiterhin signifikant höher gegenüber $\mathrm{T} 1$ bei $64,43 \pm 18,73$ und $69,12 \pm 16,73$ respektive. In der Kontrollgruppe waren die Scores an T3 und T4 nun auch signifikant höher gegenüber T1. Sie

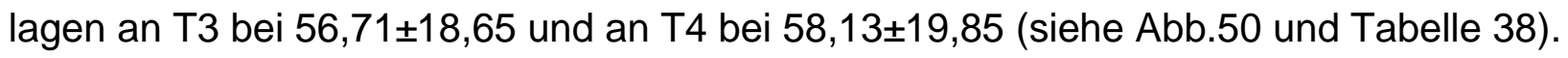

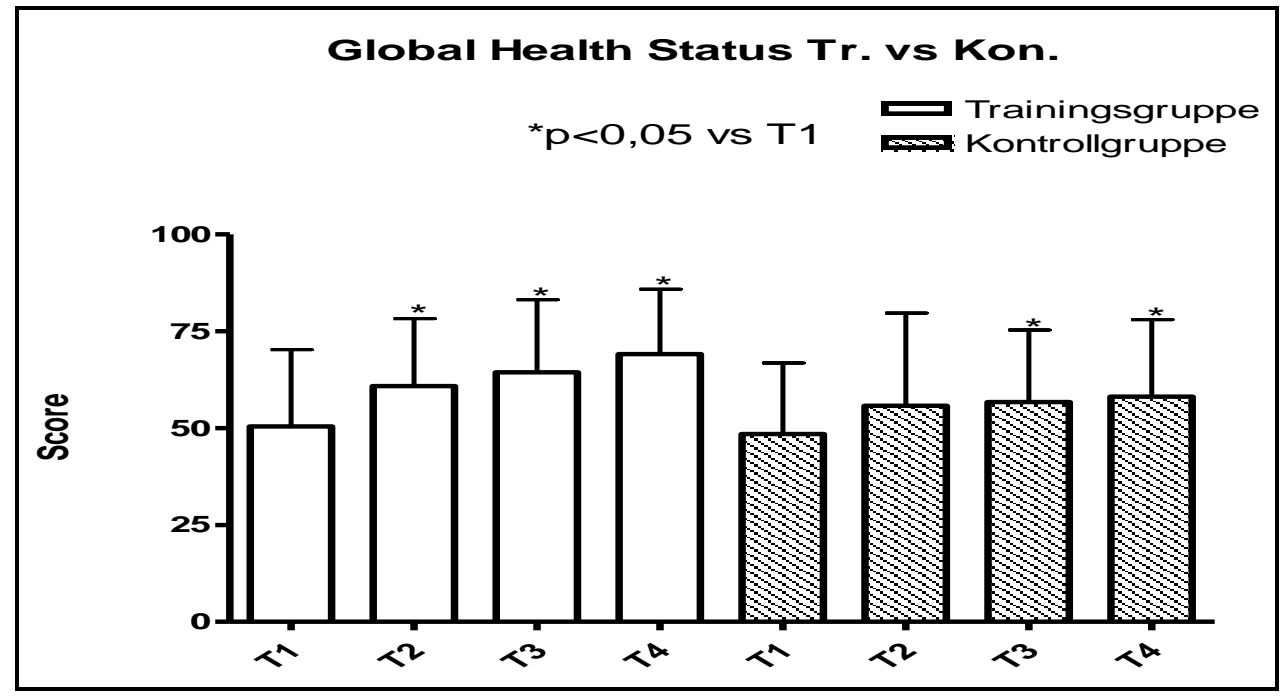

Abbildung 50: Global health status des EORTC der Trainings- und Kontrollgruppe von T1 bis T4.

\begin{tabular}{|l|l|l|l|l|l|l|l|l|}
\hline & Tr1 & Tr2 & Tr3 & Tr4 & K1 & K2 & K3 & K4 \\
\hline Mean & 50,42 & 60,88 & 64,43 & 69,12 & 48,47 & 55,78 & 56,71 & 58,13 \\
\hline SD( $\mathbf{n})$ & 19,84 & 17,36 & 18,73 & 16,73 & 18,37 & 23,95 & 18,65 & 19,85 \\
\hline
\end{tabular}

Tabelle 38: EORTC global health status der Trainings-und Kontrollgruppe von T1-T4. 


\subsubsection{Funktionsskalen des EORTC QLQ-C30}

Zum Messzeitpunkt T1 lag der Score der Trainingsgruppe des Physical functioning des EORTC QLQ-C30 Fragebogens bei 51,53 $\pm 23,5$, der Score der Kontrollgruppe bei $51,87 \pm 20,3$. Zum Messzeitpunkt T2 lag die Trainingsgruppe bei einem Score von $59,2 \pm 19,8$, die Kontrollgruppe bei 52,6 $\pm 21,8$. T3 ergab für die Trainingsgruppe einen signifikant höheren Score gegenüber T1 von 69,51 $\pm 19,9$. Auch der Score der Kontrollgruppe lag an T3 signifikant höher gegenüber T1 bei $62 \pm 21,4$. Zum Messzeitpunkt T4 lag der Score der Trainingsgruppe erneut signifikant höher gegenüber T1 bei $73,04 \pm 14,1$, der Score der Kontrollgruppe lag etwas niedriger als zu T3 bei 59,76 $\pm 22,1$ und war somit nicht mehr signifikant gegenüber T1 (siehe Abb.51a und b, sowie Tabelle 39).

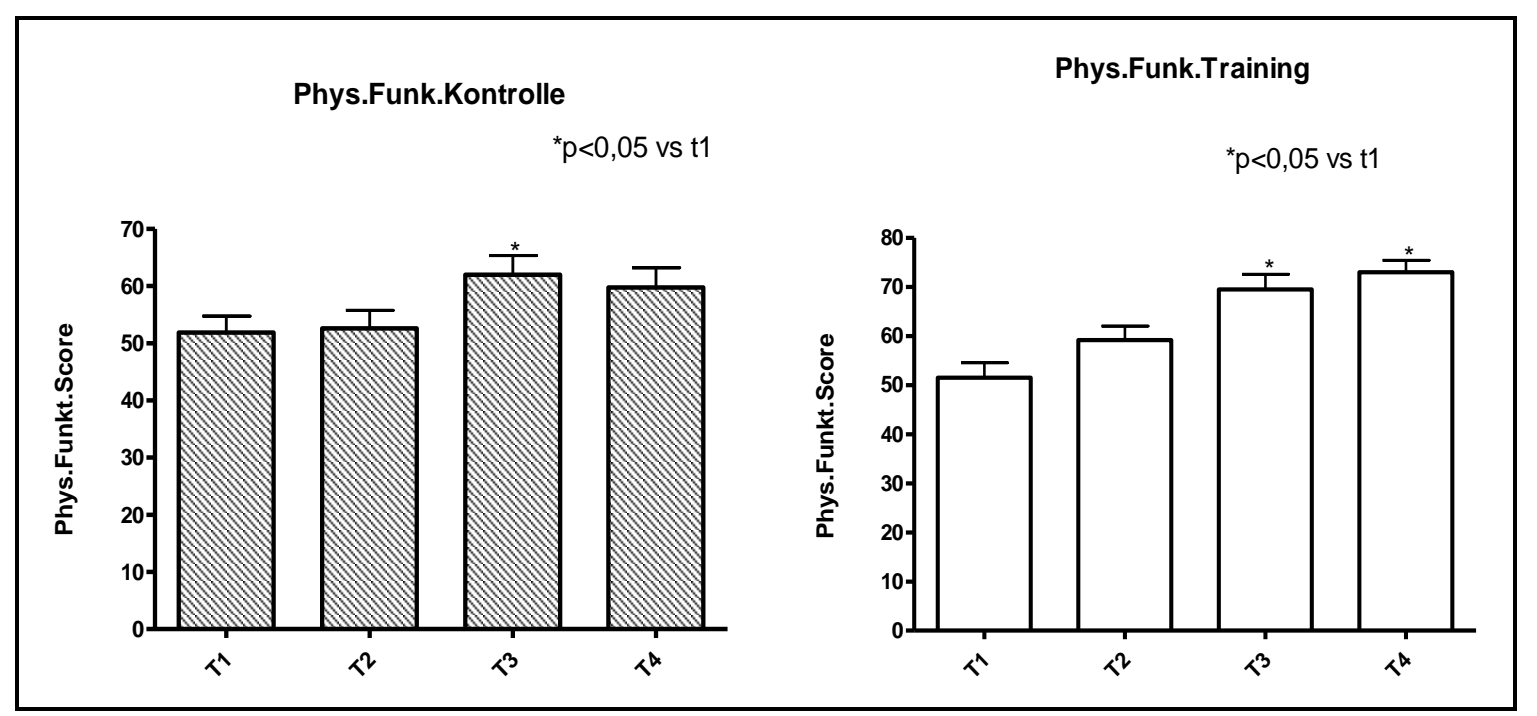

Abbildung 51 a und b: Physical Functioning des EORTC der Trainings- und Kontrollgruppe von T1 bis T4.

\begin{tabular}{|l|l|l|l|l|l|l|l|l|}
\hline & Tr1 & Tr2 & Tr3 & Tr4 & K1 & K2 & K3 & K4 \\
\hline Mean & 51,53 & 59,20 & 69,51 & 73,04 & 51,87 & 52,60 & 61,99 & 59,76 \\
\hline SD ( \pm ) & 23,49 & 19,77 & 19,86 & 14,07 & 20,29 & 21,75 & 21,41 & 22,12 \\
\hline
\end{tabular}

Tabelle 39: EORTC physical functioning-Score der Trainings- und Kontrollgruppe von T1-T4. 
Der Cognitive functioning Score (Abb.52a und b und Tabelle 40) des EORTC QLQ-C30 der Trainingsgruppe lag zu T1 bei 41 $\pm 28,4$, der Score der Kontrollgruppe bei 52,38 $\pm 28,3$. Am Messzeitpunkt T2 lag der Score der Trainingsgruppe signifikant höher gegenüber T1 bei $57,29 \pm 27,5$, der Score der Kontrollgruppe bei $57,64 \pm 32,4$. Ebenfalls signifikant höher

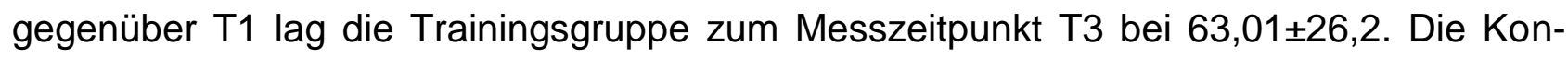
trollgruppe lag leicht erhöht gegenüber T1 und T2 bei $62,6 \pm 25,8$. An T4 lag die Trainingsgruppe weiterhin signifikant höher gegenüber T1 bei $68,14 \pm 23,7$, der Score der Kontrollgruppe blieb nahezu unverändert bei $61,79 \pm 27,7$.

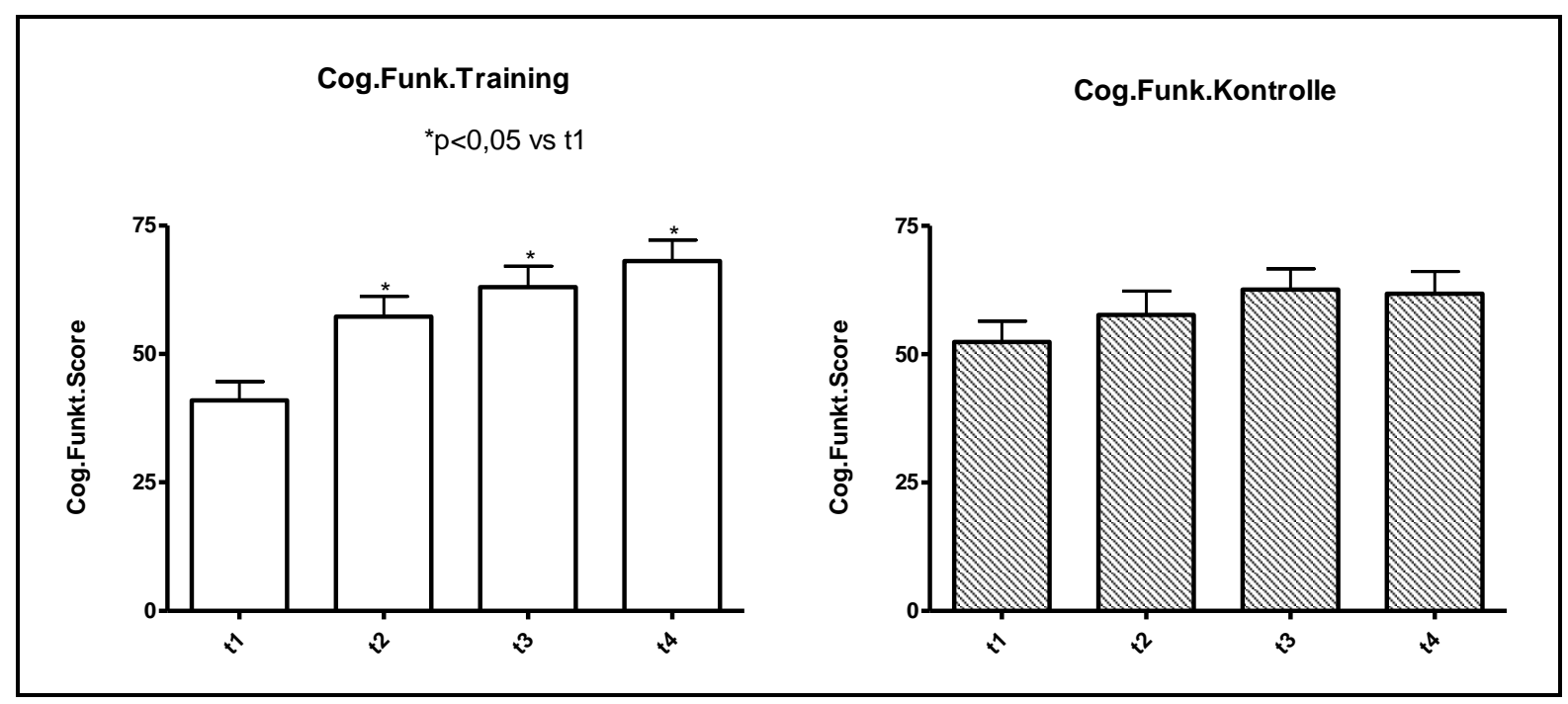

Abbildung 52 a und b: Cognitive Functioning des EORTC der Trainings- und Kontrollgruppe von T1 bis T4.

\begin{tabular}{|l|l|l|l|l|l|l|l|l|}
\hline & Tr1 & Tr2 & Tr3 & Tr4 & K1 & K2 & K3 & K4 \\
\hline Mean & 40,96 & 57,29 & 63,01 & 68,14 & 52,38 & 57,64 & 62,60 & 61,79 \\
\hline SD( \pm ) & 28,42 & 27,48 & 26,22 & 23,70 & 28,26 & 32,43 & 25,76 & 27,70 \\
\hline
\end{tabular}

Tabelle 40: EORTC cognitive functioning-Score der Trainings- und Kontrollgruppe von T1-T4. 
Der Emotional functioning Score des EORTC QLQ-C30 der Trainingsgruppe lag zu T1 bei $43,61 \pm 30,85$, der Score der Kontrollgruppe bei 57,65 $\pm 29,82$. Am Messzeitpunkt T2 lag der Score der Trainingsgruppe signifikant höher gegenüber T1 bei 62,76 $\pm 23,39$, der Score der Kontrollgruppe bei $63,37 \pm 28,49$. Ebenfalls signifikant höher gegenüber T1 lag die Trainingsgruppe zum Messzeitpunkt T3 bei 60,98 $\pm 24,33$. Die Kontrollgruppe lag unterhalb T1 und T2 bei 55,69 $\pm 26,38$. An T4 lag die Trainingsgruppe weiterhin signifikant höher gegenüber T1 bei 64,95 $\pm 23,64$, der Score der Kontrollgruppe fiel weiter gegenüber den vorherigen Messzeitpunkten auf 37,40 $\pm 26,65$ ab (siehe Abb. 53 und Tabelle 41).

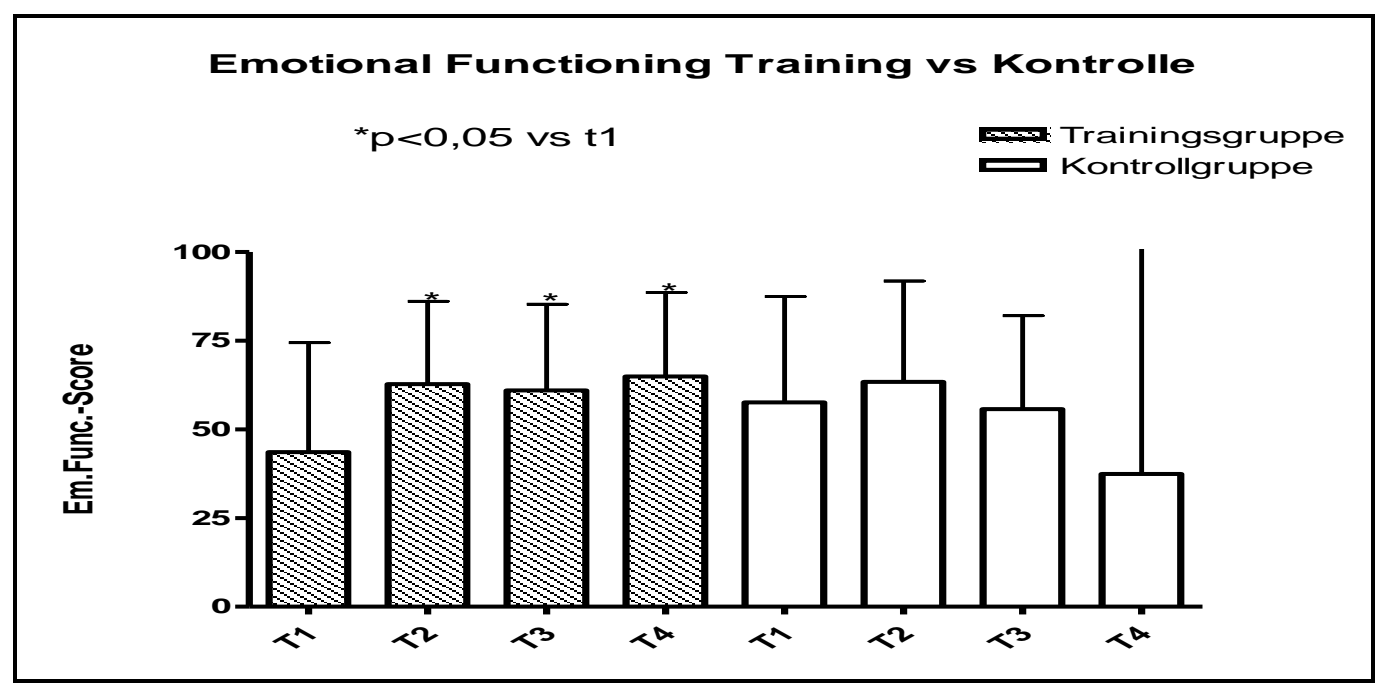

Abbildung 53: Emotional Functioning des EORTC der Trainings- und Kontrollgruppe von T1 bis T4.

\begin{tabular}{|l|l|l|l|l|l|l|l|l|}
\hline & Tr1 & Tr2 & Tr3 & Tr4 & K1 & K2 & K3 & K4 \\
\hline Mean & 43,61 & 62,76 & 60,98 & 64,95 & 57,65 & 63,37 & 55,69 & 37,40 \\
\hline SD( \pm ) & 30,85 & 23,39 & 24,33 & 23,64 & 29,82 & 28,49 & 26,38 & 26,65 \\
\hline
\end{tabular}

Tabelle 41: EORTC emotional functioning-Score der Trainings- und Kontrollgruppe von T1-T4. 
Zum Messzeitpunkt T1 kam die Trainingsgruppe im role functioning auf einen Score von $43,33 \pm 31,77$, die Kontrollgruppe auf 46,26 $\pm 30,29$. Zum Messzeitpunkt T2 gab es in der Trainingsgruppe bereits eine signifikante Steigerung des Scores auf $61,90 \pm 33,68$. In der Kontrollgruppe gab es eine leichte Steigerung auf $55,90 \pm 27,18$. Ebenfalls waren die Scores der Trainingsgruppe an T3 und T4 im Vergleich zu T1 signifikant höher bei $65,04 \pm 26,56$ und 68,63 $\pm 26,52$ respektive. In der Kontrollgruppe stieg der Score signifikant von T1 nach T3 auf 59,35 $\pm 30,74$ an und dann weiter nach T4 auf 60,98 $\pm 31,32$ (siehe Abb. 54 und Tabelle 42).

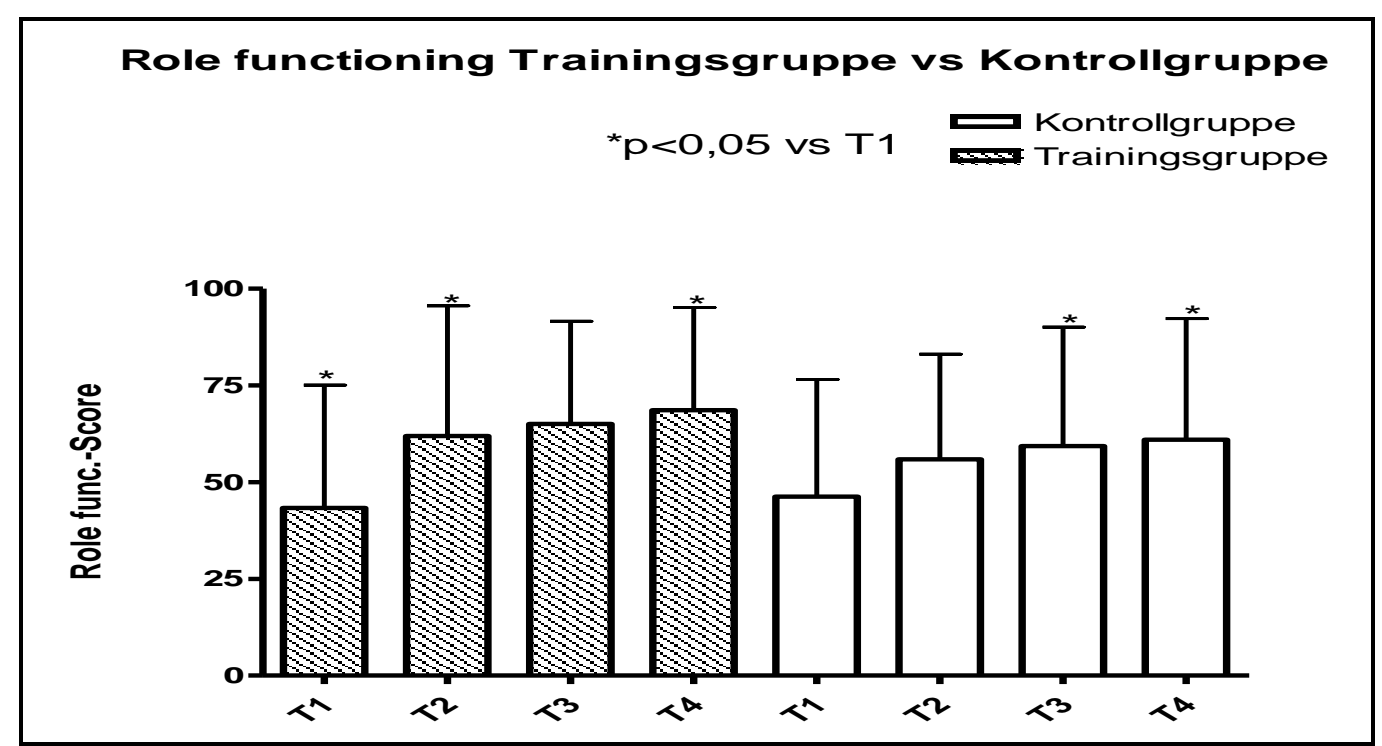

Abbildung 54: Role Functioning des EORTC der Trainings- und Kontrollgruppe von T1 bis T4.

\begin{tabular}{|l|l|l|l|l|l|l|l|l|}
\hline & Tr1 & Tr2 & Tr3 & Tr4 & K1 & K2 & K3 & K4 \\
\hline Mean & 43,33 & 61,90 & 65,04 & 68,63 & 46,26 & 55,90 & 59,35 & 60,98 \\
\hline SD( \pm ) & 31,77 & 33,68 & 26,56 & 26,52 & 30,29 & 27,18 & 30,74 & 31,32 \\
\hline
\end{tabular}

Tabelle 42: EORTC role functioning-Score der Trainings- und Kontrollgruppe von T1-T4. 
Beim social functioning des EORTC (Abb. 55 und Tabelle 43) hatte die Trainingsgruppe zum Messzeitpunkt T1einen Score von 47,22 $\pm 33,64$, die Kontrollgruppe von 53,75 $\pm 29,32$. In der Trainingsgruppe kam es zu einem signifikanten Anstieg zum MesszeitpunktT2 auf $65,99 \pm 32,45$. Die Kontrollgruppe verzeichnete einen nicht signifikanten Anstieg des Scores auf $64,24 \pm 30,36$. Weiterhin signifikant höher blieben die Scores der Trainingsgrup-

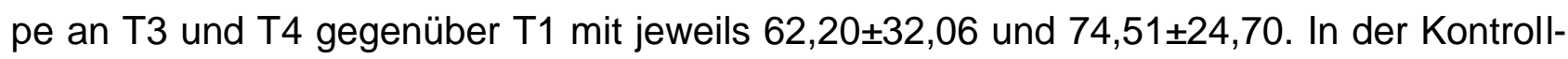
gruppe kam es zum Messzeitpunkt T3 zu keiner signifikanten Änderung. Der Score lag bei $63,82 \pm 28,60$ fast genauso hoch wie zu T2. Eine signifikante Änderung gab es dann an T4. Hier war der Score der Kontrollgruppe mit 69,51 $\pm 29,56$ signifikant höher als an T1.

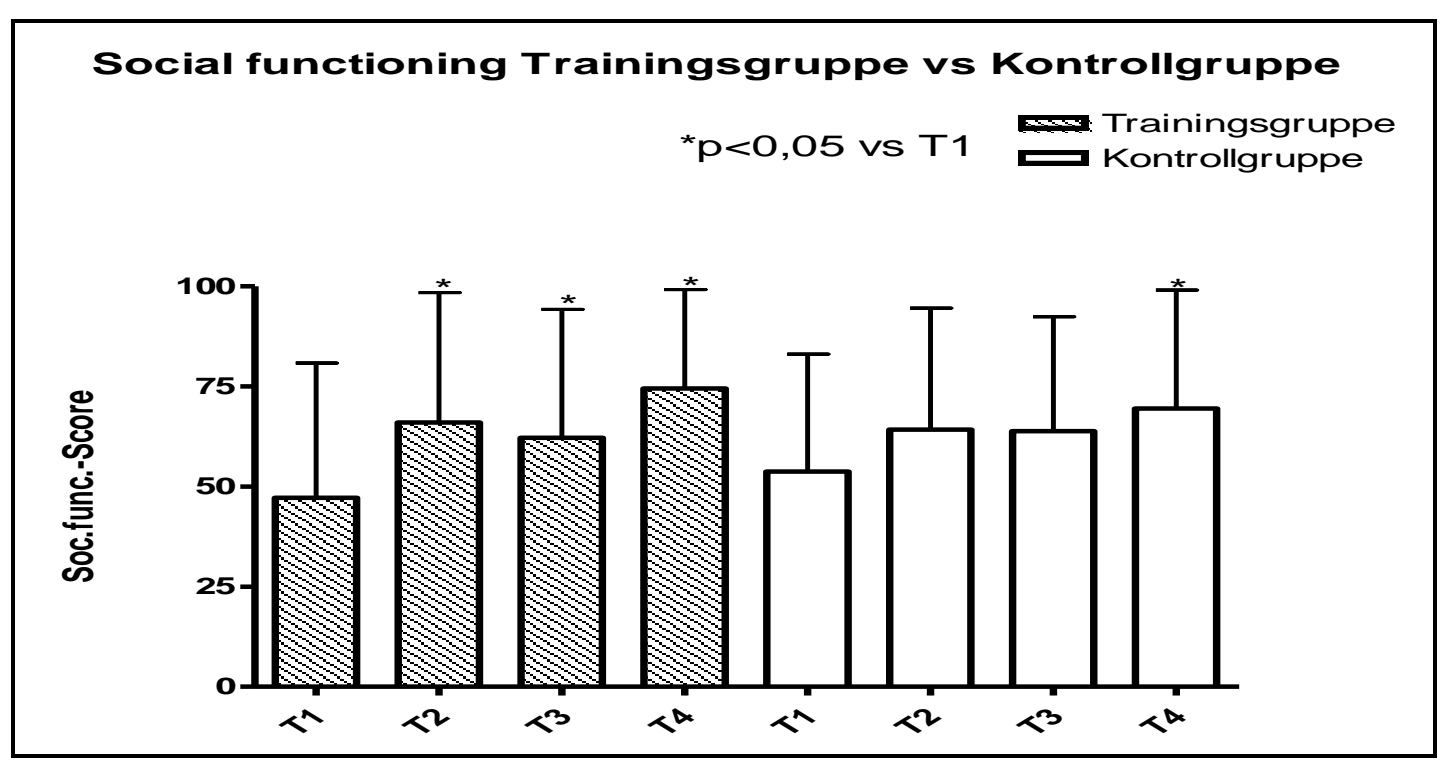

Abbildung 55: Social Functioning des EORTC der Trainings- und Kontrollgruppe von T1 bis T4.

\begin{tabular}{|l|l|l|l|l|l|l|l|l|}
\hline & Tr1 & Tr2 & Tr3 & Tr4 & K1 & K2 & K3 & K4 \\
\hline Mean & 47,22 & 65,99 & 62,20 & 74,51 & 53,74 & 64,24 & 63,82 & 69,51 \\
\hline SD( \pm ) & 33,64 & 32,45 & 32,06 & 24,70 & 29,32 & 30,36 & 28,60 & 29,56 \\
\hline
\end{tabular}

Tabelle 43: EORTC social functioning-Score der Trainings- und Kontrollgruppe von T1-T4. 


\subsubsection{Symptomskalen des EORTC QLQ-C30}

Der Fatigue Score des EORTC-Fragebogens (Abb.56 und Tabelle 44) ergab für die Trainingsgruppe an T1 einen Wert von 68,36 $\pm 23,87$ und für die Kontrollgruppe einen Score von 65,31 $\pm 22,06$. Zum Messzeitpunkt T2 lag der Score der Trainingsgruppe bei $53,24 \pm 23,48$, der der Kontrollgruppe bei $54,14 \pm 24,26$. Die Fatigue Scores der Trainings-

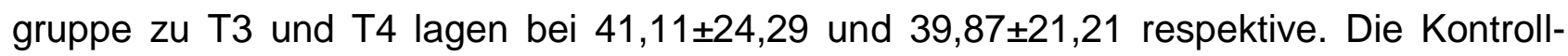
gruppe hatte an T3 einen Score von 53,93 $\pm 24,92$, an T4 einen Score von 50,68 $\pm 24,35$.

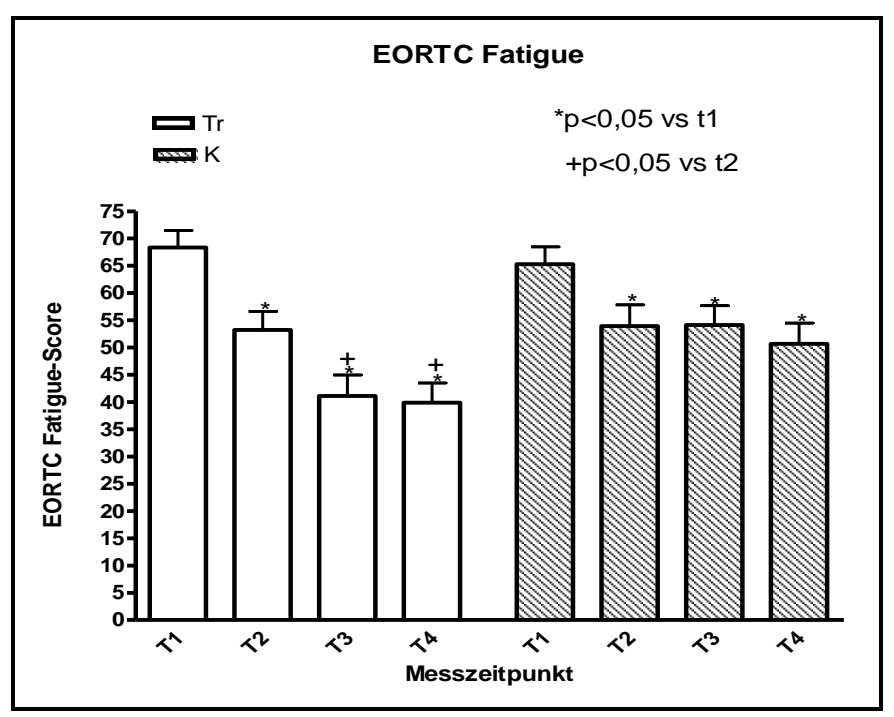

Abbildung 56: Graphen der EORTC-Fatigue der Trainings- und Kontrollgruppe von T1 bis T4.

\begin{tabular}{|l|l|l|l|l|l|l|l|l|}
\hline & Tr1 & Tr2 & Tr3 & Tr4 & K1 & K2 & K3 & K4 \\
\hline Mean & 68,36 & 53,24 & 41,11 & 39,87 & 65,31 & 54,14 & 53,93 & 50,68 \\
\hline SD( \pm ) & 23,87 & 23,48 & 24,29 & 21,21 & 22,06 & 24,26 & 24,92 & 24,35 \\
\hline
\end{tabular}

Tabelle 44: EORTC Fatigue-Score der Trainings- und Kontrollgruppe von T1-T4. 
Die Werte für Erbrechen des EORTC lagen in beiden Gruppen zu allen Messterminen in einem nicht relevanten Bereich. An T1 lag der Score der Trainingsgruppe bei 8,61 $\pm 15,49$. Der Wert der Kontrollgruppe an T1 lag bei 6,12 $\pm 12,59$. Signifikant niedriger lag der Score der Trainingsgruppe an T2 bei 2,04 $\pm 8,07$. Der Score der Kontrollgruppe an T2 lag nicht signifikant niedriger bei 3,12 $\pm 9,51$. An T3 lag der Wert der Trainingsgruppe bei 4,88 $\pm 11,33$ nicht mehr signifikant niedriger gegenüber T1. An T4 war er es wieder mit 1,96 $\pm 5,45$. In der Kontrollgruppe gab es weiterhin keine signifikanten Änderungen. Der Score an T3 lag

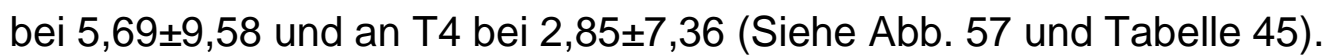

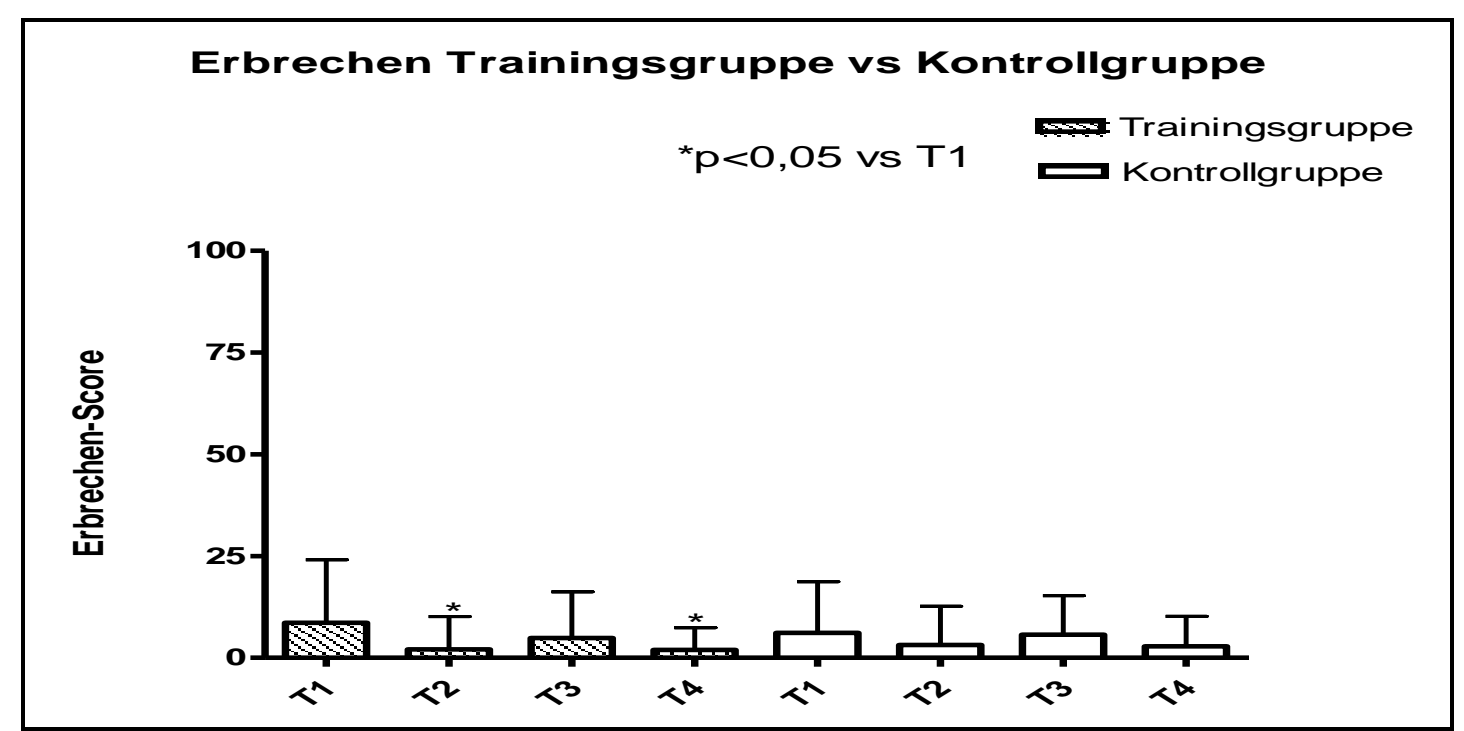

Abbildung 57: Nausea and vomiting des EORTC der Trainings- und Kontrollgruppe von T1 bis T4.

\begin{tabular}{|l|l|l|l|l|l|l|l|l|}
\hline & Tr1 & Tr2 & Tr3 & Tr4 & K1 & K2 & K3 & K4 \\
\hline Mean & 8,611 & 2,041 & 4,878 & 1,961 & 6,122 & 3,125 & 5,691 & 2,846 \\
\hline SD( $\mathbf{(})$ & 15,49 & 8,074 & 11,33 & 5,451 & 12,59 & 9,513 & 9,581 & 7,362 \\
\hline
\end{tabular}

Tabelle 45: Nausea und vomiting (Erbrechen) der Trainings- und Kontrollgruppe von T1-T4. 
In beiden Gruppen gab es beim Schmerzscore keine signifikanten Veränderungen über die gesamte Studiendauer (siehe Abb.58 und Tabelle 46). Die Trainingsgruppe lag an T1 bei 36,11 $\pm 31,32$ und an T2 bei $29,25 \pm 31,09$. Die Kontrollgruppe lag an T1 bei $43,88 \pm 32,33$ und an T2 bei 30,33.

Zum Messzeitpunkt T3 lag der Score der Trainingsgruppe bei 36,59 $\pm 33,17$. Der Wert der Kontrollgruppe lag zu diesem Zeitpunkt bei 40,24 27,13 . An T4 lag die Trainingsgruppe

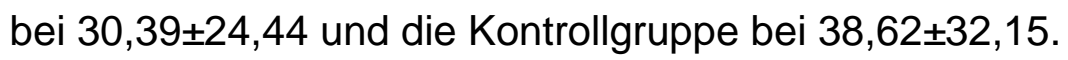

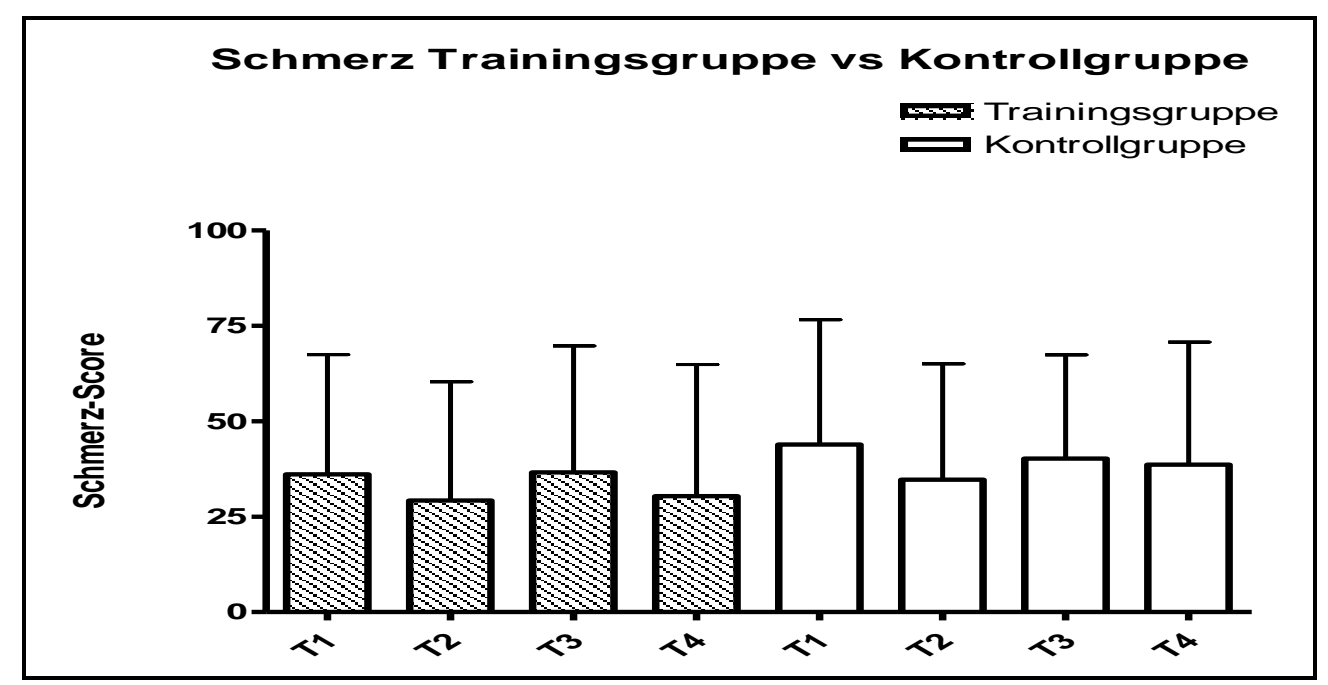

Abbildung 58: Painscore (Schmerzen) des EORTC der Trainings- und Kontrollgruppe von T1 bis T4.

\begin{tabular}{|l|l|l|l|l|l|l|l|l|}
\hline & Tr1 & Tr2 & Tr3 & Tr4 & K1 & K2 & K3 & K4 \\
\hline Mean & 36,11 & 29,25 & 36,59 & 30,39 & 43,88 & 34,72 & 40,24 & 38,62 \\
\hline SD( \pm ) & 31,32 & 31,09 & 33,17 & 34,44 & 32,75 & 30,33 & 27,13 & 32,15 \\
\hline
\end{tabular}

Tabelle 46: Pain (Schmerzen) der Trainings- und Kontrollgruppe von T1-T4. 
Der Score für Schlaflosigkeit lag bei der Trainingsgruppe zum Messzeitpunkt T1 bei $65,00 \pm 36,01$ und in der Kontrollgruppe bei 52,38 $\pm 36,00$. Zum Messzeitpunkt T2 kam es in der Trainingsgruppe zu einer signifikanten Änderung gegenüber T1. Der Wert lag an T2 bei 50,69 $\pm 37,02$ und auch an T3 mit 48,78 $\pm 36,59$ signifikant niedriger. Der Wert der Kontrollgruppe lag an T2 bei 45,83 $\pm 32,01$ nicht signifikant niedriger und an T3 bei 43,90 $\pm 32,01$ ebenfalls nicht. An T4 war der Wert der Trainingsgruppe erneut signifikant niedriger gegenüber T1 bei 46,08 $\pm 33,85$. Der Wert der Kontrollgruppe lag an T4 bei 47,15 $\pm 37,25$ (siehe Abb.59 und Tabelle 47).

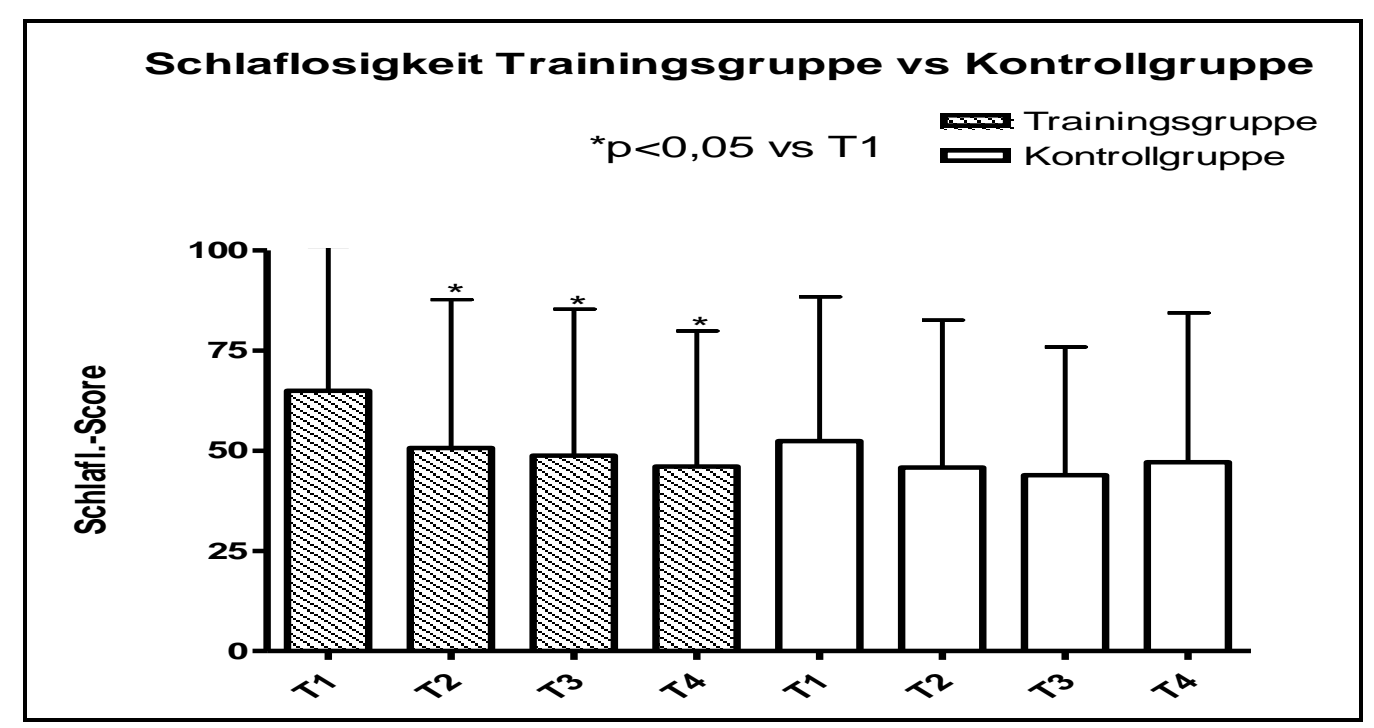

Abbildung 59: Insomnia (Schlaflosigkeit/Schlafstörungen) Score des EORTC der Trainings- und Kontrollgruppe von T1 bis T4.

\begin{tabular}{|l|l|l|l|l|l|l|l|l|}
\hline & Tr1 & Tr2 & Tr3 & Tr4 & K1 & K2 & K3 & K4 \\
\hline Mean & 65,00 & 50,69 & 48,78 & 46,08 & 52,38 & 45,83 & 43,90 & 47,15 \\
\hline SD( \pm ) & 36,01 & 37,02 & 36,59 & 33,85 & 36,00 & 36,79 & 32,01 & 37,25 \\
\hline
\end{tabular}

Tabelle 47: Insomnia (Schlaflosigkeit) der Trainings- und Kontrollgruppe von T1-T4. 
Der Wert für Dyspnoe lag in der Trainingsgruppe zum ersten Messzeitpunkt T1 bei $36,11 \pm 30,25$ und in der Kontrollgruppe bei $39,46 \pm 36,43$. Keine signifikanten Veränderungen gab es in beiden Gruppen gegenüber T2. In der Trainingsgruppe lag dieser Wert bei $31,25 \pm 30,25$ und in der Kontrollgruppe bei $31,97 \pm 33,30$. Signifikant niedriger lagen die Werte beider Gruppen an T3 im Vergleich zu T1. Die Trainingsgruppe lag hier bei $23,33 \pm 25,26$ und die Kontrollgruppe bei 25,20 $\pm 27,67$. Erneut signifikant niedriger war der Score der Trainingsgruppe an T4 im Vergleich zu T1 mit 16,67 $\pm 3,57$. Der Wert der Kontrollgruppe lag an T4 bei 27,64 $\pm 29,72$ (siehe Abb. 60 und Tabelle 48).

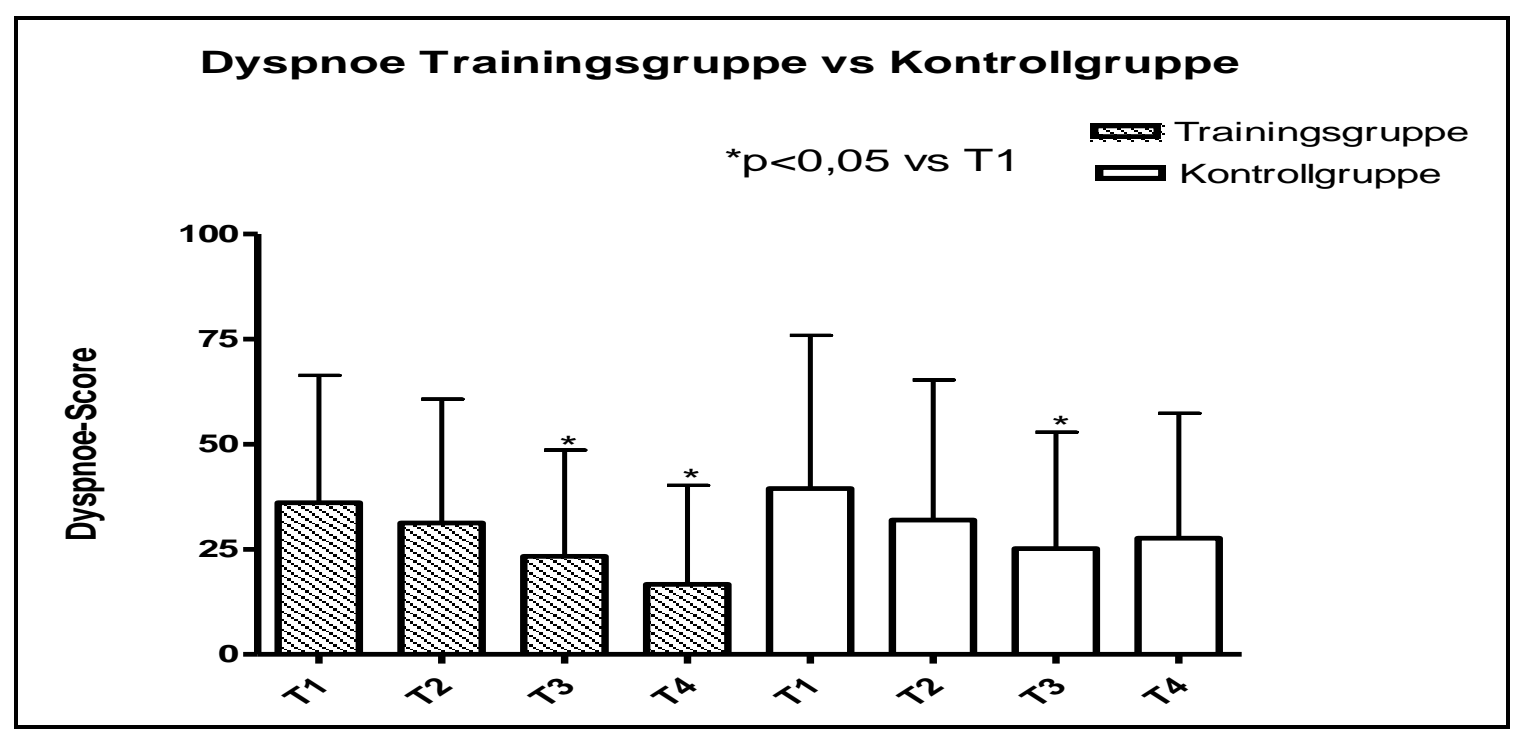

Abbildung 60: Dyspnoe des EORTC der Trainings- und Kontrollgruppe von T1 bis T4.

\begin{tabular}{|l|l|l|l|l|l|l|l|l|}
\hline & Tr1 & Tr2 & Tr3 & Tr4 & K1 & K2 & K3 & K4 \\
\hline Mean & 36,11 & 31,25 & 23,33 & 16,67 & 39,46 & 31,97 & 25,20 & 27,64 \\
\hline SD( $\mathbf{(})$ & 30,25 & 29,50 & 25,26 & 23,57 & 36,43 & 33,30 & 27,67 & 29,72 \\
\hline
\end{tabular}

Tabelle 48: Dyspnoe der Trainings- und Kontrollgruppe von T1-T4. 
Der Score für den Appetitverlust lag in der Trainingsgruppe zum Messzeitpunkt T1 bei

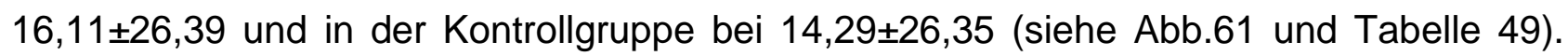
Zum Zeitpunkt T2 lag der Score der Trainingsgruppe bei 9,72 $\pm 21,70$ und der Score der Kontrollgruppe bei 11,11 $\pm 23,15$. An T3 lag der Wert der Trainingsgruppe bei 13,82 $\pm 25,79$ und an T4 signifikant niedriger gegenüber T1 bei 5,82 $\pm 17,35$. Der Score der Kontrollgrup-

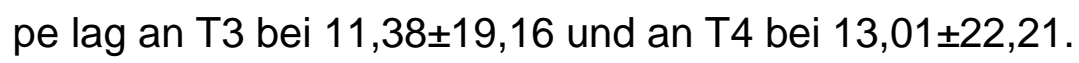

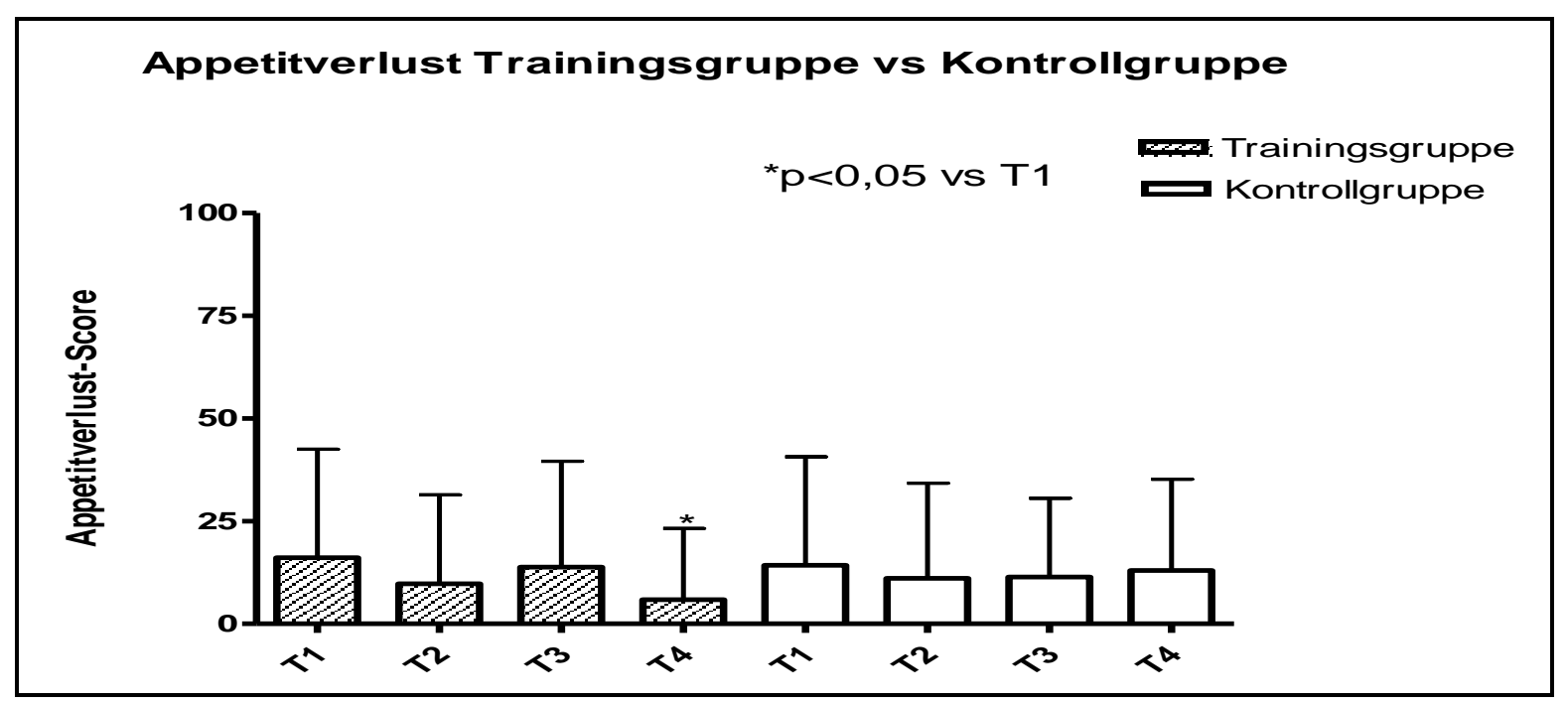

Abbildung 61: Appetite loss (Appetitverlust) des EORTC der Trainings- und Kontrollgruppe von T1 bis T4.

\begin{tabular}{|l|l|l|l|l|l|l|l|l|}
\hline & Tr1 & Tr2 & Tr3 & Tr4 & K1 & K2 & K3 & K4 \\
\hline Mean & 16,11 & 9,722 & 13,82 & 5,882 & 14,29 & 11,11 & 11,38 & 13,01 \\
\hline (SD \pm ) & 26,39 & 21,70 & 25,79 & 17,35 & 26,35 & 23,15 & 19,16 & 22,21 \\
\hline
\end{tabular}

Tabelle 49: Appetite loss (Appetitverlust) der Trainings- und Kontrollgruppe von T1-T4. 
Keine signifikanten Veränderungen gab es bei den Scores für constipation (Abb.62 und Tabelle 50), die Scores der Trainingsgruppe lag an $\mathrm{T} 1$ bei $12,43 \pm 22,23$ und an $\mathrm{T} 2$ bei $6,12 \pm 17,58$. Die Werte der Kontrollgruppe lagen bei $14,97 \pm 28,10$ an $T 1$ und bei $14,58 \pm 24,70$ an T2. Zum Messzeitpunkt T3 lag der Score in der Trainingsgruppe bei $7,32 \pm 17,50$, in der Kontrollgruppe lag dieser Wert bei 13,01 $\pm 25,69$. Die Messung an T4

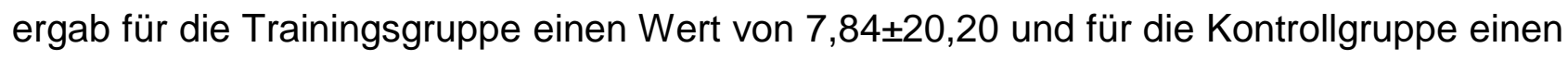
Wert von $17,89 \pm 26,97$.

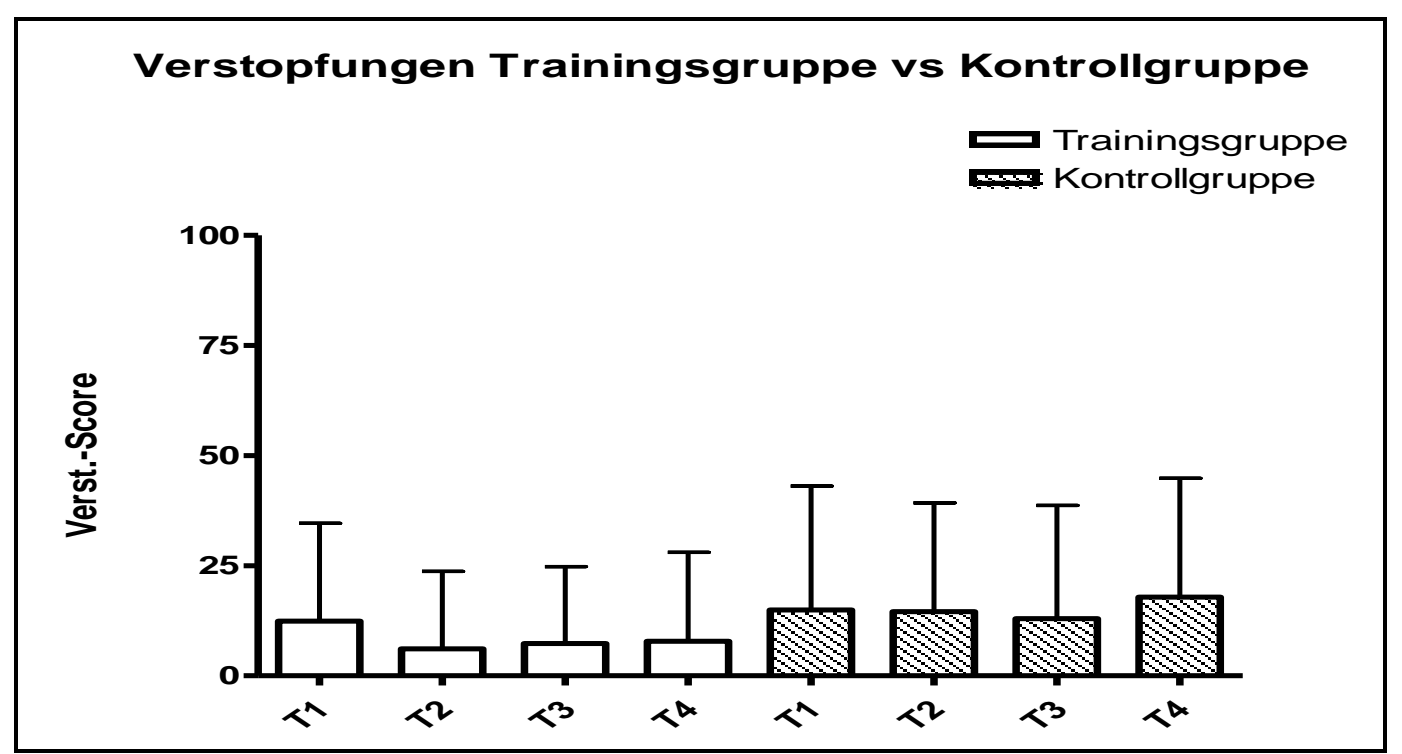

Abbildung 62: Constipation (Verstopfungen) des EORTC der Trainings- und Kontrollgruppe von T1 bis T4.

\begin{tabular}{|l|l|l|l|l|l|l|l|l|}
\hline & Tr1 & Tr2 & Tr3 & Tr4 & K1 & K2 & K3 & K4 \\
\hline Mean & 12,43 & 6,122 & 7,317 & 7,843 & 14,97 & 14,58 & 13,01 & 17,89 \\
\hline SD( \pm ) & 22,23 & 17,58 & 17,50 & 20,20 & 28,10 & 24,70 & 25,69 & 26,97 \\
\hline
\end{tabular}

Tabelle 50: Constipation (Verstopfungen) der Trainings- und Kontrollgruppe von T1-T4. 
Am Messzeitpunkt T1 lag der Score der Trainingsgruppe für Diarrhoe bei 9,60 $\pm 21,49$, der Score der Kontrollgruppe bei 3,40 $\pm 12,26$. An T2 lag der Wert der Trainingsgruppe etwas niedriger bei 7,48 $\pm 21,80$ und in der Kontrollgruppe bei $4,17 \pm 11,14$ etwas höher. Zum Zeitpunkt T3 lag der Wert der Trainingsgruppe bei 6,50 $\pm 17,03$ und an T4 signifikant niedriger gegenüber $T 1$ bei $0,98 \pm 5,72$. Die Werte der Kontrollgruppe lagen an $T 3$ bei $4,88 \pm 17,58$

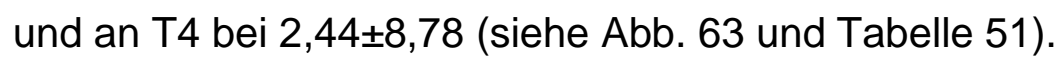

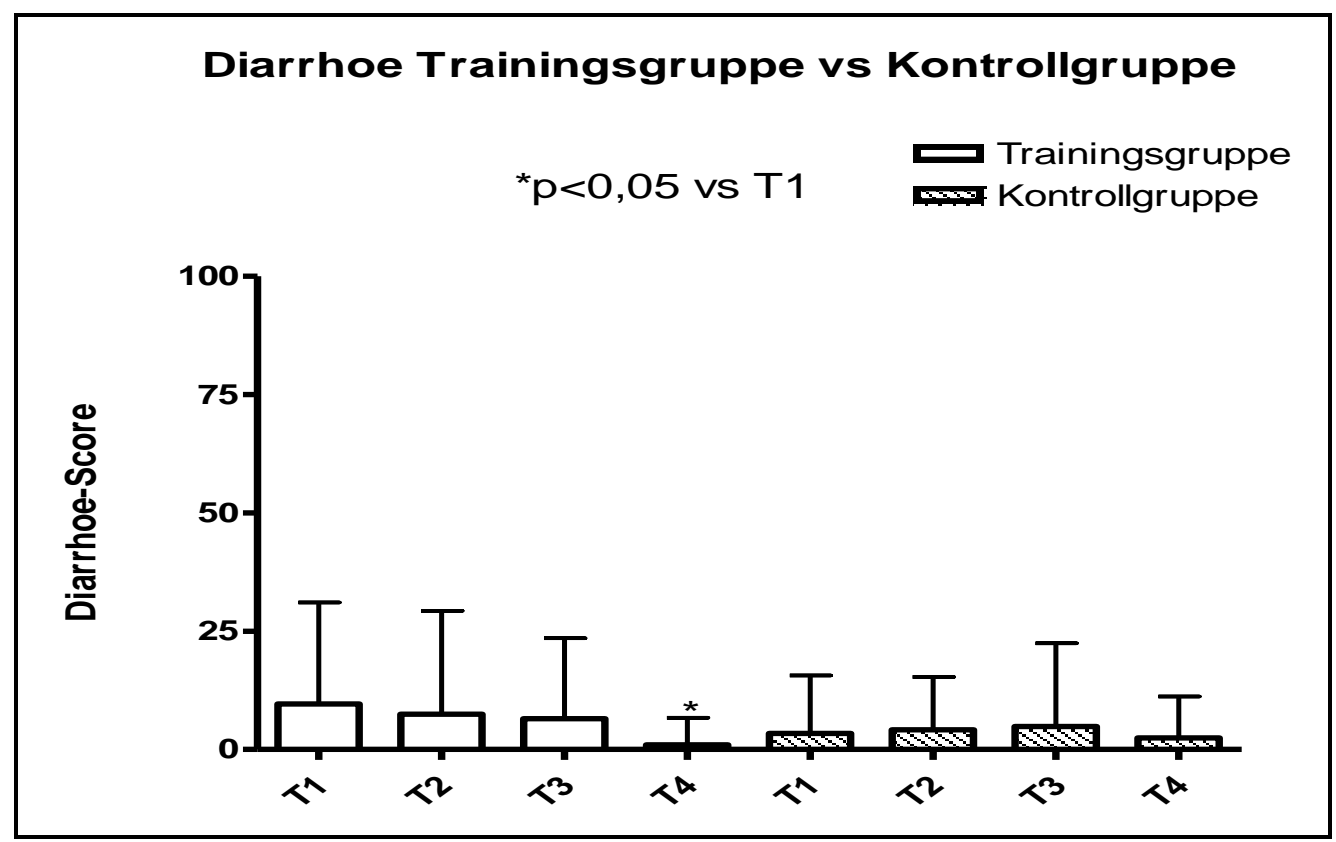

Abbildung 63: Diarrhoe des EORTC der Trainings- und Kontrollgruppe von T1 bis T4.

\begin{tabular}{|l|l|l|l|l|l|l|l|l|}
\hline & Tr1 & Tr2 & Tr3 & Tr4 & K1 & K2 & K3 & K4 \\
\hline Mean & 9,605 & 7,483 & 6,504 & 0,9804 & 3,401 & 4,167 & 4,878 & 2,439 \\
\hline SD( $\mathbf{n})$ & 21,49 & 21,80 & 17,03 & 5,717 & 12,26 & 11,14 & 17,58 & 8,788 \\
\hline
\end{tabular}

Tabelle 51: Diarrhoe der Trainings- und Kontrollgruppe von T1-T4. 
Der Wert für finanzielle Schwierigkeiten des EORTC (siehe Abb. 64 und Tabelle 52) lag zum Zeitpunkt T1 für die Trainingsgruppe bei 32,18 $\pm 34,18$ und für die Kontrollgruppe bei $30,56 \pm 30,62$. Zum Zeitpunkt T2 lag der Wert der Trainingsgruppe bei $21,09 \pm 30,20$, der Score der Kontrollgruppe lag bei $29,08 \pm 35,86$. An T3 lag der Wert für die Trainingsgruppe

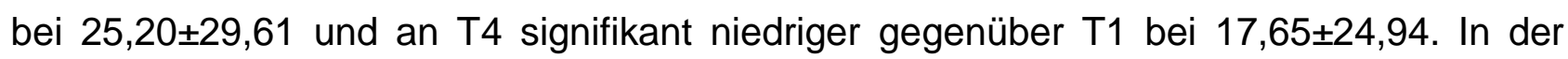
Kontrollgruppe lag der Score an T3 bei 26,02 $\pm 36,14$ und an T4 bei 22,76 $\pm 35,31$.

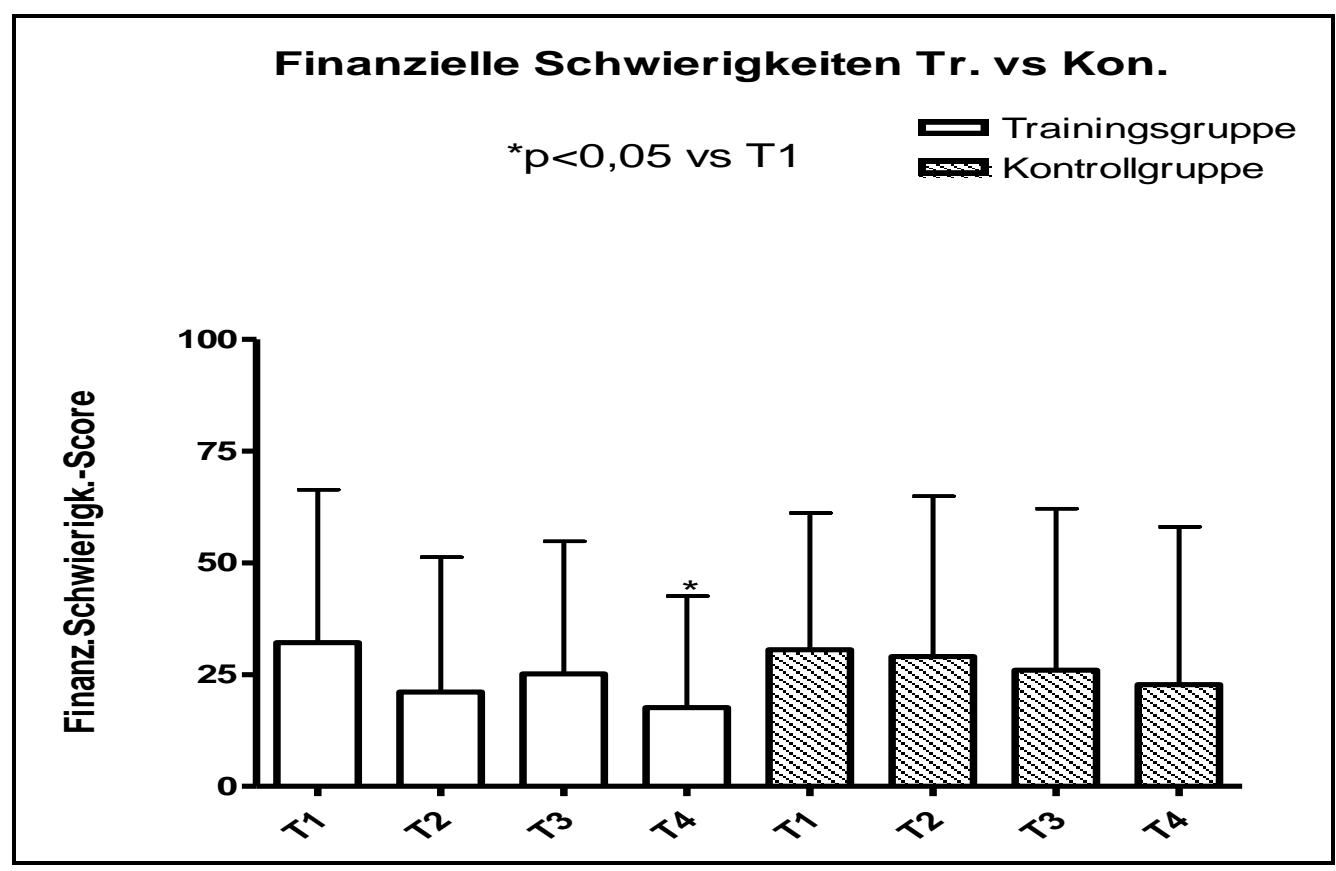

Abbildung 64: Financial difficulties (finanzielle Schwierigkeiten) des EORTC der Trainings- und Kontrollgruppe von T1 bis T4.

\begin{tabular}{|l|l|l|l|l|l|l|l|l|}
\hline & Tr1 & Tr2 & Tr3 & Tr4 & K1 & K2 & K3 & K4 \\
\hline Mean & 32,18 & 21,09 & 25,20 & 17,65 & 30,56 & 29,08 & 26,02 & 22,76 \\
\hline SD( \pm ) & 34,18 & 30,20 & 29,61 & 24,94 & 30,62 & 35,86 & 36,14 & 35,31 \\
\hline
\end{tabular}

Tabelle 52: Financial difficulties (finanzielle Schwierigkeiten) der Trainings- und Kontrollgruppe von T1-T4. 


\subsection{Ergebnisse des HADS}

Der Angstscore der Trainingsgruppe lag an $\mathrm{T} 1$ bei $8,8 \pm 4,2$. Der Depressionsscore lag bei $7,0 \pm 4,1$. Zum Messzeitpunkt T2 lag der Angstscore der Trainingsgruppe mit 7,2 $\pm 3,9$ signifikant niedriger gegenüber T1. Der Depressionsscore lag zu T2 bei 5,9 $\pm 3,2$. Zu den Messzeitpunkten T3 und T4 lagen die Angstscores der Trainingsgruppe jeweils bei 7,1 $\pm 3,8$ und

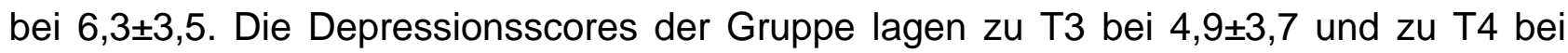
$4,0 \pm 3,1$ und somit signifikant niedriger gegenüber dem Messzeitpunkt T1 (siehe Abb. 65ad und Tabelle 53).

In der Kontrollgruppe lag der Angstscore zum ersten Messzeitpunkt T1 bei $8,4 \pm 4,5$ und der Depressionsscore bei 7,1 $\pm 4,9$. An T2 lag der Angstscore der Kontrollgruppe bei 6,9 $\pm 4,4$, der Depressionsscore lag bei 5,5 $\pm 4,4$. Zu den Messzeiten T3 und T4 änderte sich der Angstscore nur minimal auf 7,3 $\pm 4,2$ an T3 und 7,1 $\pm 4,2$ an T4. Auch der Depressionsscore änderte sich nur minimal auf 5,6 $\pm 4,4$ an T3 und 5,9 $\pm 5,1$ an T4.
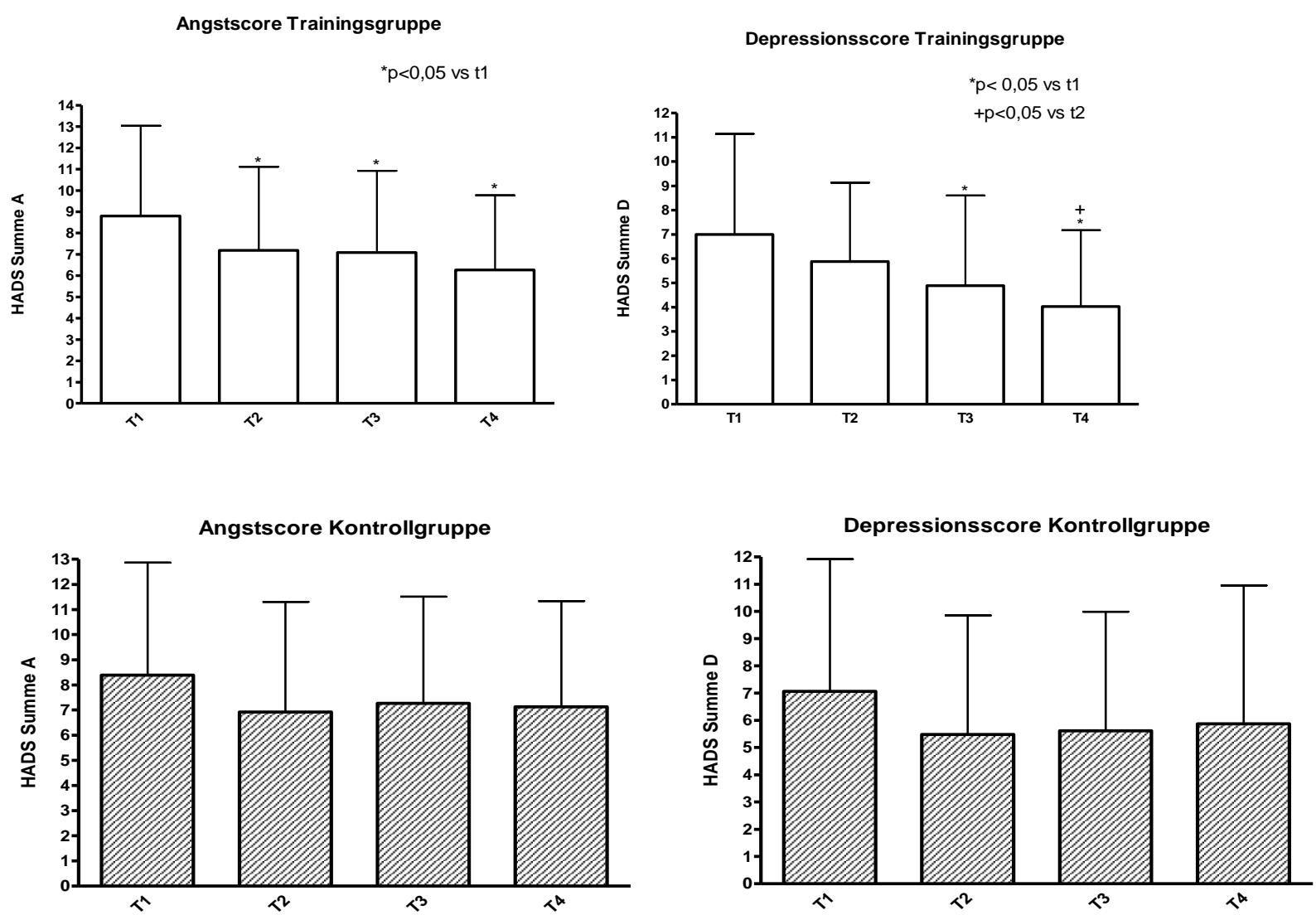

Abbildung 65 a-d: Angst- und Depressionsscores der Trainings- und Kontrollgruppe von T1 bis T4. 


\begin{tabular}{|l|l|l|}
\hline Angstscore T1 & $8,80 \pm 4,23$ & $8,39 \pm 4,48$ \\
\hline Angstscore T2 & $7,18 \pm 3,93$ & $6,92 \pm 4,38$ \\
\hline Angstscore T3 & $7,08 \pm 3,84$ & $7,27 \pm 4,24$ \\
\hline Angstscore T4 & $6,27 \pm 3,51$ & $7,13 \pm 4,21$ \\
\hline Depr.Score T1 & $7,0 \pm 4,15$ & $7,06 \pm 4,86$ \\
\hline Depr.Score T2 & $5,89 \pm 3,24$ & $5,48 \pm 4,38$ \\
\hline Depr.Score T3 & $4,89 \pm 3,72$ & $5,61 \pm 4,38$ \\
\hline Depr.Score T4 & $4,03 \pm 3,15$ & $5,88 \pm 5,10$ \\
\hline
\end{tabular}

Tabelle 53: Angst- und Depressionsscores der Trainings- und Kontrollgruppe von T1 bis T4. Werte sind dargestellt in Mean \pm SD

\subsection{Trainingsumfang und Fatigue}

Der allgemeine Fatiguescore gemessen durch den MFI lag bei der Gruppe, die unterdurchschnittlich trainierte nach drei Monaten Training zum Messzeitpunkt T3 bei 40,5 $\pm 24,23$. Der Score der durchschnittlich Trainierenden lag bei 53,5 $\pm 19,86$ und der der

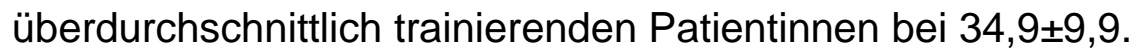

Sechs Monate nach der Rehabilitation, also nach drei weiteren Trainingsmonaten lag der Wert der unterdurchschnittlich trainierenden Patientinnengruppe bei $52,42 \pm 16,66$. Die durchschnittlich trainierende Gruppe hatte einen allgemeinen Fatiguescore von $38,29 \pm 22,43$ und die überdurchschnittlich Trainierenden lagen bei einem Score von $53,80 \pm 19,97$.

Die Schnittdefinition fand bereits auf Seite 22, Tabelle 3, statt (siehe Abb. 66a und b und Tabelle 54).

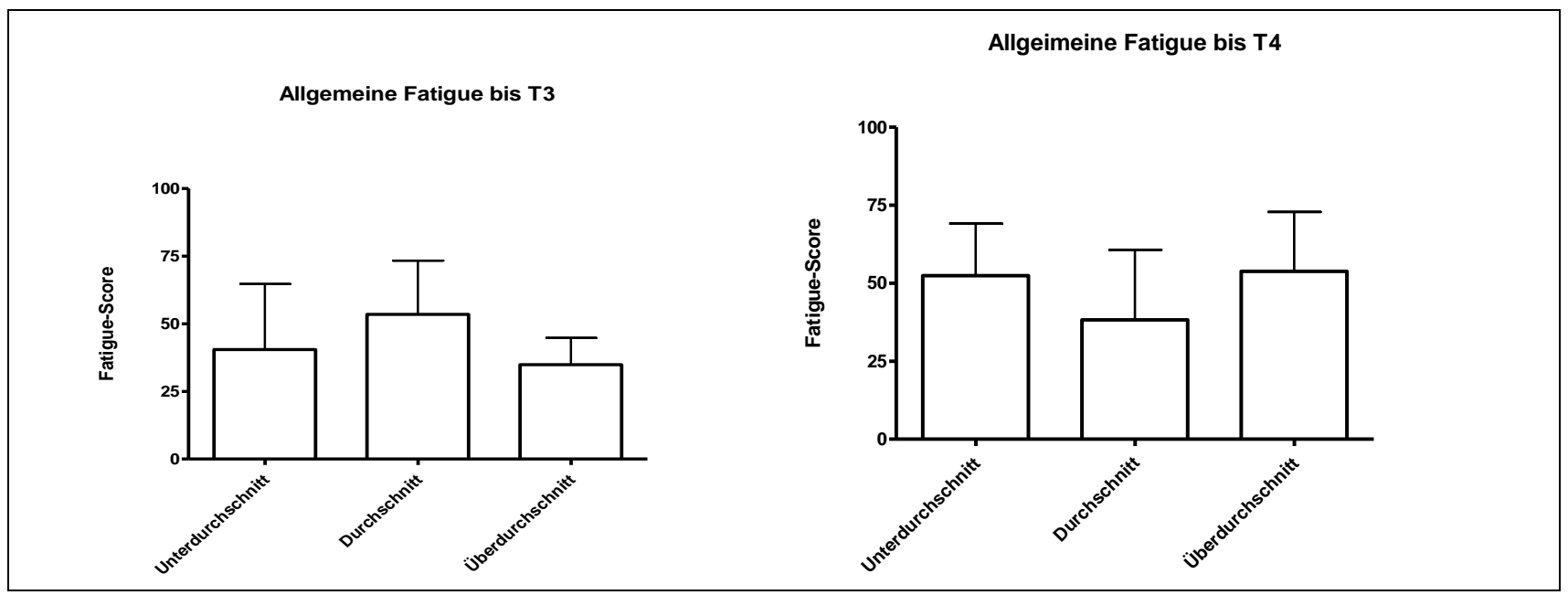

Abbildung 66 a und b: Allgemeine Fatigue bezogen auf den Trainingsumfang. 


\begin{tabular}{|l|l|l|}
\hline Unterdurchschnitt & $40,5 \pm 24,23$ & $52,42 \pm 16,66$ \\
\hline Durchschnitt & $53,5 \pm 19,86$ & $38,29 \pm 22,43$ \\
\hline Überdurchschnitt & $34,9 \pm 9,9$ & $53,80 \pm 19,07$ \\
\hline
\end{tabular}

Tabelle 54: Durchschnittliche Trainingsumfänge in den Monaten 1-3 (bisT3) und 4-6 (bisT4) nach Rehabilitation und ihr Bezug auf die allgemeine Fatigue.

Der Score der physischen Fatigue lag bei den unterdurchschnittlich Trainierenden nach drei Monaten bei $28,75 \pm 20,20$. Die durchschnittlich trainierenden Patientinnen hatten einen Score von 44,73 $\pm 15,53$ und die überdurchschnittlich Trainierenden einen Score von $26,70 \pm 17,20$.

Drei Monate später, also sechs Monate postrehabilitativ hatten die Patientinnen, die unterdurchschnittlich trainierten einen Score von 32,18 14,29 und die durchschnittlich Trainierenden einen Score von $28,00 \pm 18,17$. Die Patientinnen mit einem überdurchschnittlichen

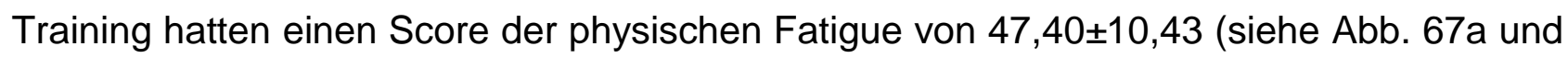
b, sowie Tabelle 55).

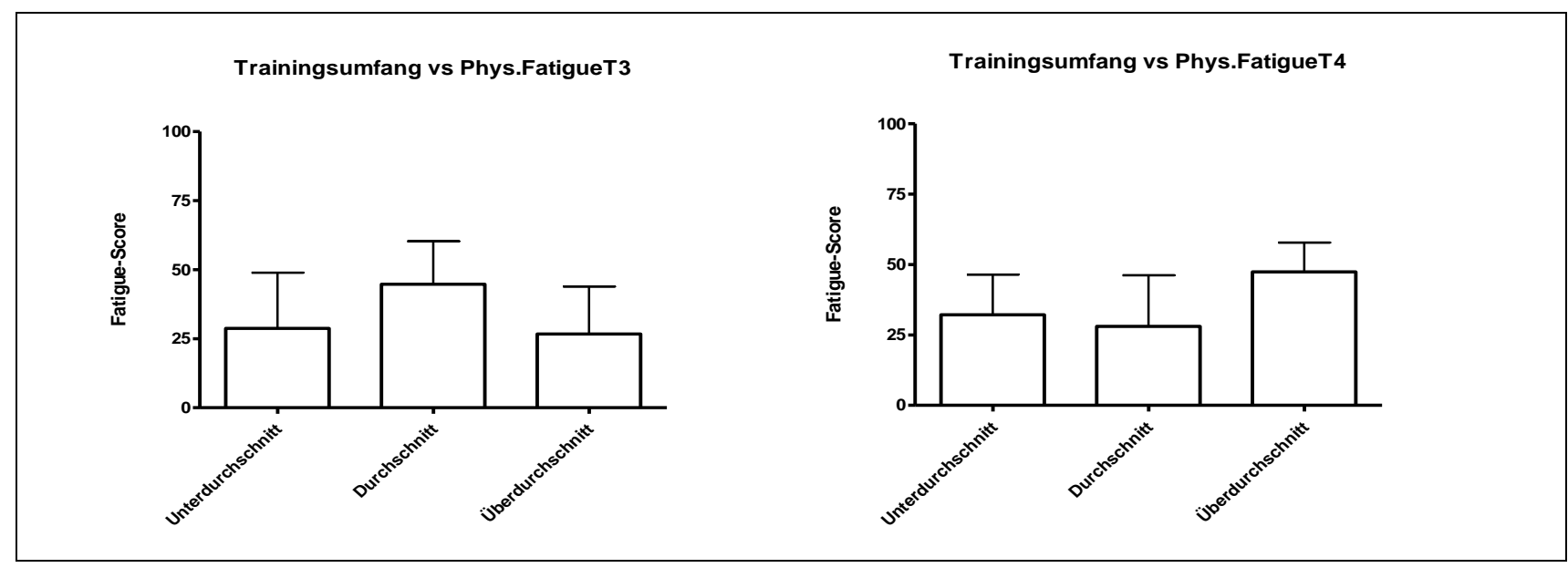

Abbildung 67 a und b: Physische Fatigue bezogen auf den Trainingsumfang.

\begin{tabular}{|l|l|l|}
\hline & Physische Fatigue bis T3 & Physische Fatigue bis T4 \\
\hline Unterdurchschnitt & $28,75 \pm 20,20$ & $32,18 \pm 14,29$ \\
\hline Durchschnitt & $44,73 \pm 15,53$ & $28,00 \pm 18,17$ \\
\hline Überdurchschnitt & $26,70 \pm 17,20$ & $47,40 \pm 10,43$ \\
\hline
\end{tabular}

Tabelle 55: Durchschnittliche Trainingsumfänge in den Monaten 1-3 (bisT3) und 4-6 (bisT4) nach Rehabilitation und ihr Bezug auf die physische Fatigue.

Die oben angeführten Vergleiche zwischen den Trainingsumfängen und den jeweiligen Ebenen der Fatigue sollten auf Grund der Dokumentationsweise der Ausdauersportarten durch die Teilnehmerinnen mit Vorsicht interpretiert werden, da eine einheitliche Zuordnung der einzelnen Parameter wie Trainingsumfänge, Dauer und Intensität der Ausdauer- 
sportarten nicht immer möglich war. Eine genauere Begriffserklärung der Grundbegriffe zur Belastungsgestaltung befindet sich in den Anlagen (Anlage 8).

\subsection{Ergebnisse der Kraftmessungen}

Die Trainingsgruppe erreichte an T1 für das rechte Bein einen Maximalkraftwert von $226,2 \pm 55,2 \mathrm{~N}$, für das linke Bein kam die Gruppe auf $237,6 \pm 44,8 \mathrm{~N}$. Die Kraftmessung an T2 ergab für das rechte Bein einen Maximalkraftwert von $253,4 \pm 49,7 \mathrm{~N}$, für das linke Bein wurde eine Maximalkraft von 258,4 $\pm 76,7 \mathrm{~N}$ gemessen (siehe Abb. 68a und b).

Die isometrische Kraftmessung der Beine ergab zum Messzeitpunkt T1 in der Kontrollgruppe für das rechte Bein einen Wert von $234 \pm 86,7 \mathrm{~N}$, für das linke Bein einen Wert von $231,7 \pm 94 \mathrm{~N}$. An T2 lag die Maximalkraft des rechten Beines der Kontrollgruppe bei $246,4 \pm 70,6 \mathrm{~N}$, die des linken Beines bei 246,3 $\pm 78 \mathrm{~N}$.

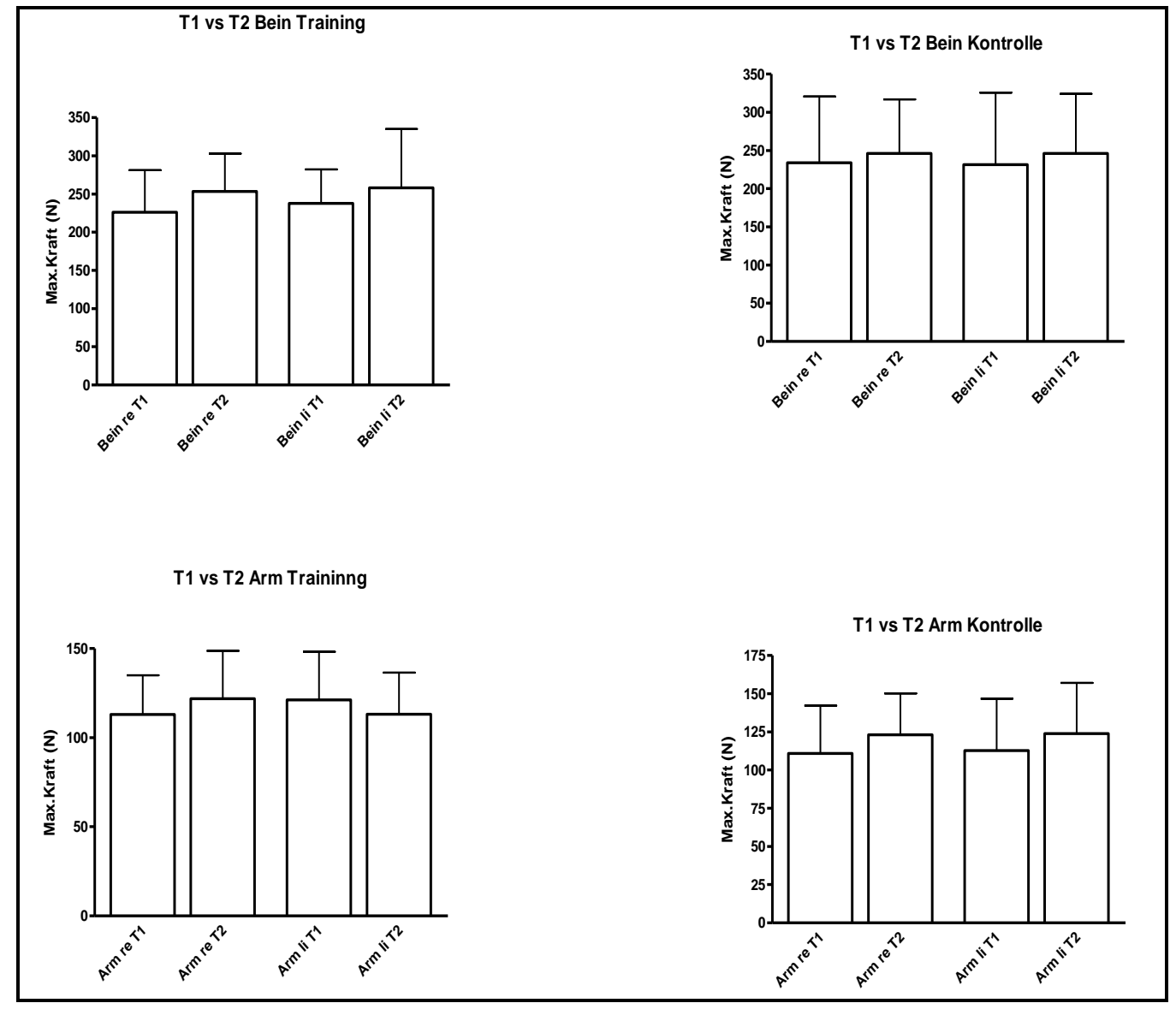

Abbildung 68 a-d: Darstellung der isometrischen Kraftmessung der Beinstrecker und Armbeuger zu Beginn und Ende (T1 und T2) der Rehabilitation in der Trainings- und Kontrollgruppe.

Die Trainingsgruppe erreichte an T1 eine Maximalkraft im rechten Arm von 113,1 $\pm 21,9 \mathrm{~N}$, Links lag diese bei $121,2 \pm 26,7 \mathrm{~N}$. An T2 lag der Wert der Maximalkraft für den rechten Arm 
bei $121,8 \pm 26,7 \mathrm{~N}$, für den linken Arm sogar niedriger gegenüber dem ersten Messzeitpunkt bei $113,2 \pm 23,3 \mathrm{~N}$ (siehe Abbildung 68c und d).

Die Maximalkraftmessung für den rechten Arm ergab am Messzeitpunkt T1 in der Kontrollgruppe einen Wert von $110,8 \pm 31,3 \mathrm{~N}$, der linke Arm lag bei $112,9 \pm 34 \mathrm{~N}$. Zum Messzeitpunkt T2 erreichte die Gruppe eine Maximalkraft des rechten Armes von 123,1 $\pm 27 \mathrm{~N}$, im linken Arm lag dieser Wert bei 123,8 $\pm 33,4 \mathrm{~N}$.

\subsection{Ergebnisse der Leistungsdiagnostik}

Die Abbildung 69 zeigt die Ergebnisse des 6 Min. Gehtests im Vergleich der Trainings- zur Kontrollgruppe. Die Kontrollgruppe hatte eine signifikante Verbesserung der zurückgelegten Strecke während 6 minütigem Gehen von T1 $(510,7 \mathrm{~m})$ nach T2 $(540,6 \mathrm{~m})$. In der Trainingsgruppe gab es eine leichte, aber nicht signifikante Verbesserung von $\mathrm{T} 1(535,2 \mathrm{~m})$ nach T2 (553m).

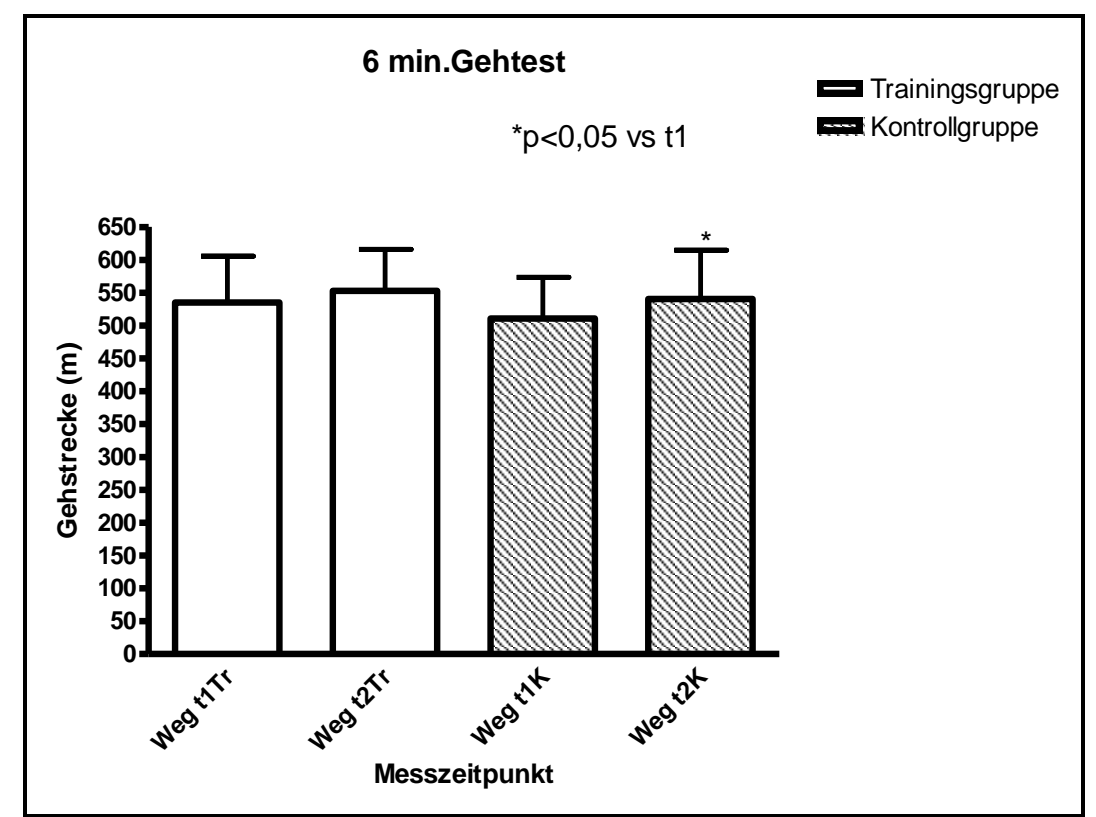

Abbildung 69: Wegstrecke des 6 Min. Gehtests zu Anfang und zum Ende der Rehabilitation in der Trainings- und Kontrollgruppe. 
In den Abbildungen 70a und b, sowie in Tabelle 56 sind die einzelnen Wattstufen an den beiden Messzeitpunkten T1 und T2 der Trainingsgruppe dargestellt. Es ist zu sehen, dass der Puls (Herzfrequenz) zusammen mit der Wattzahl ansteigt. Deutlich gleichmäßiger findet der Herzfrequenzanstieg zum Messzeitpunkt T2 (Abb. 70b) statt.

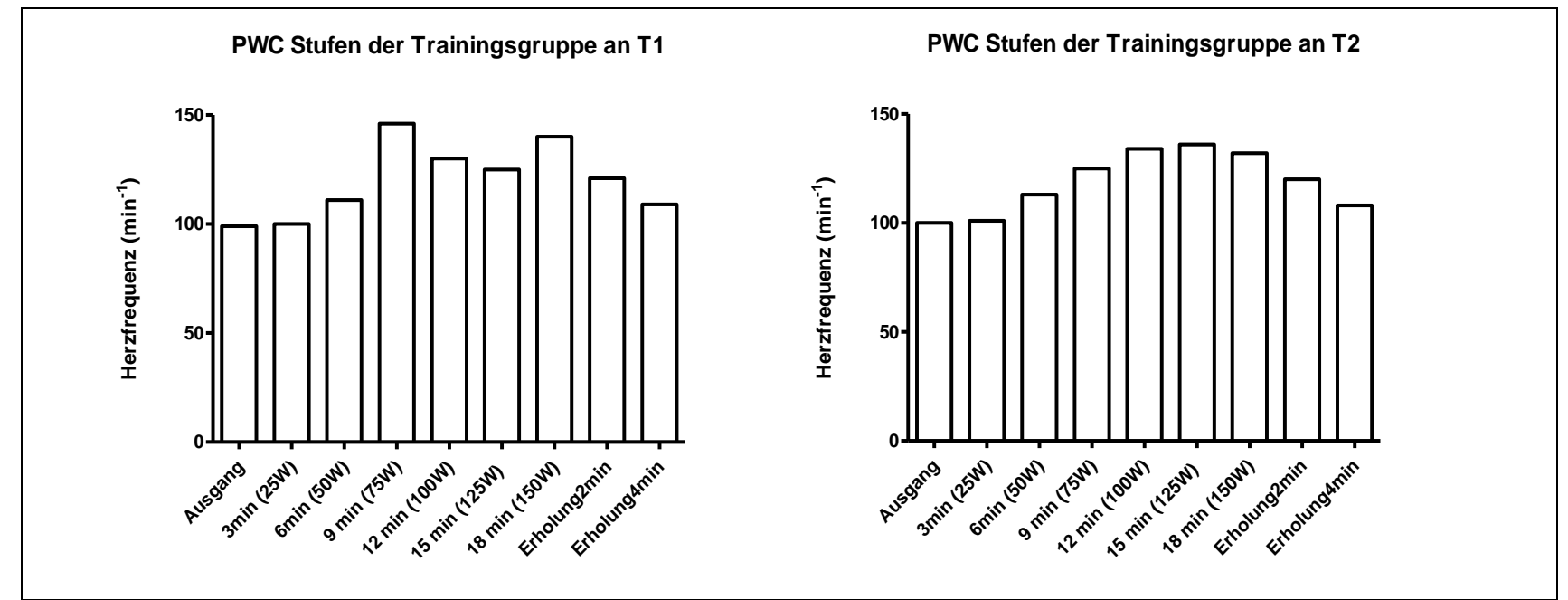

Abbildung 70 a und b: Pulsfrequenzen der Trainingsgruppe an den Messzeitpunkten T1und T2 während unterschiedlicher Wattstufen.

\begin{tabular}{|l|l|l|l|l|l|l|l|l|l|}
\hline & Ausgang & $25 W$ & $50 W$ & $75 W$ & $100 W$ & $125 W$ & $150 W$ & Erh.2min. & Erh.4min. \\
\hline TrT1 & $\begin{array}{l}99 \\
(n=60)\end{array}$ & $\begin{array}{l}100 \\
(n=60)\end{array}$ & $\begin{array}{l}111 \\
(n=59)\end{array}$ & $\begin{array}{l}146 \\
(n=49)\end{array}$ & $\begin{array}{l}130 \\
(n=27)\end{array}$ & $\begin{array}{l}125 \\
(n=10)\end{array}$ & $\begin{array}{l}140 \\
(n=3)\end{array}$ & $\begin{array}{l}121 \\
(n=60)\end{array}$ & $\begin{array}{l}109 \\
(n=60)\end{array}$ \\
\hline TrT2 & 100 & 101 & 113 & 125 & 134 & 136 & 132 & 120 & 108 \\
& $(n=52)$ & $(n=52)$ & $(n=52)$ & $(n=45)$ & $(n=26)$ & $(n=7)$ & $(n=2)$ & $(n=52)$ & $(n=52)$ \\
\hline KT1 & $\begin{array}{l}(n) \\
(n=54)\end{array}$ & $\begin{array}{l}93 \\
(n=54)\end{array}$ & $\begin{array}{l}103 \\
(n=54)\end{array}$ & $\begin{array}{l}115 \\
(n=46)\end{array}$ & $\begin{array}{l}126 \\
(n=27)\end{array}$ & $\begin{array}{l}135 \\
(n=12)\end{array}$ & $\begin{array}{l}145 \\
(n=1)\end{array}$ & $\begin{array}{l}111 \\
(n=54)\end{array}$ & $\begin{array}{l}99 \\
(n=54)\end{array}$ \\
\hline KT2 & $\begin{array}{l}95 \\
(n=51)\end{array}$ & $\begin{array}{l}95 \\
(n=49)\end{array}$ & $\begin{array}{l}105 \\
(n=47)\end{array}$ & $\begin{array}{l}119 \\
(n=45)\end{array}$ & $\begin{array}{l}130 \\
(n=26)\end{array}$ & $\begin{array}{l}137 \\
(n=9)\end{array}$ & $\begin{array}{l}143 \\
(n=3)\end{array}$ & $\begin{array}{l}114 \\
(n=51)\end{array}$ & $\begin{array}{l}102 \\
(n=51)\end{array}$ \\
\hline
\end{tabular}

Tabelle 56: Pulsfrequenzen (Mittelwerte, berechnet aus der jeweiligen Teilnehmerinnenanzahl $n$ ) der Trainings- und Kontrollgruppe während des PWC-Tests vom Start bis zur Erholung nach 4 Minuten. 
Die Abbildungen 71a und $\mathrm{b}$ und Tabelle 56 zeigen die Herzfrequenz der Kontrollgruppe während des PWC- Tests. An beiden Messzeitpunkten war ein gleichmäßiger Herzfrequenzanstieg zu verzeichnen.

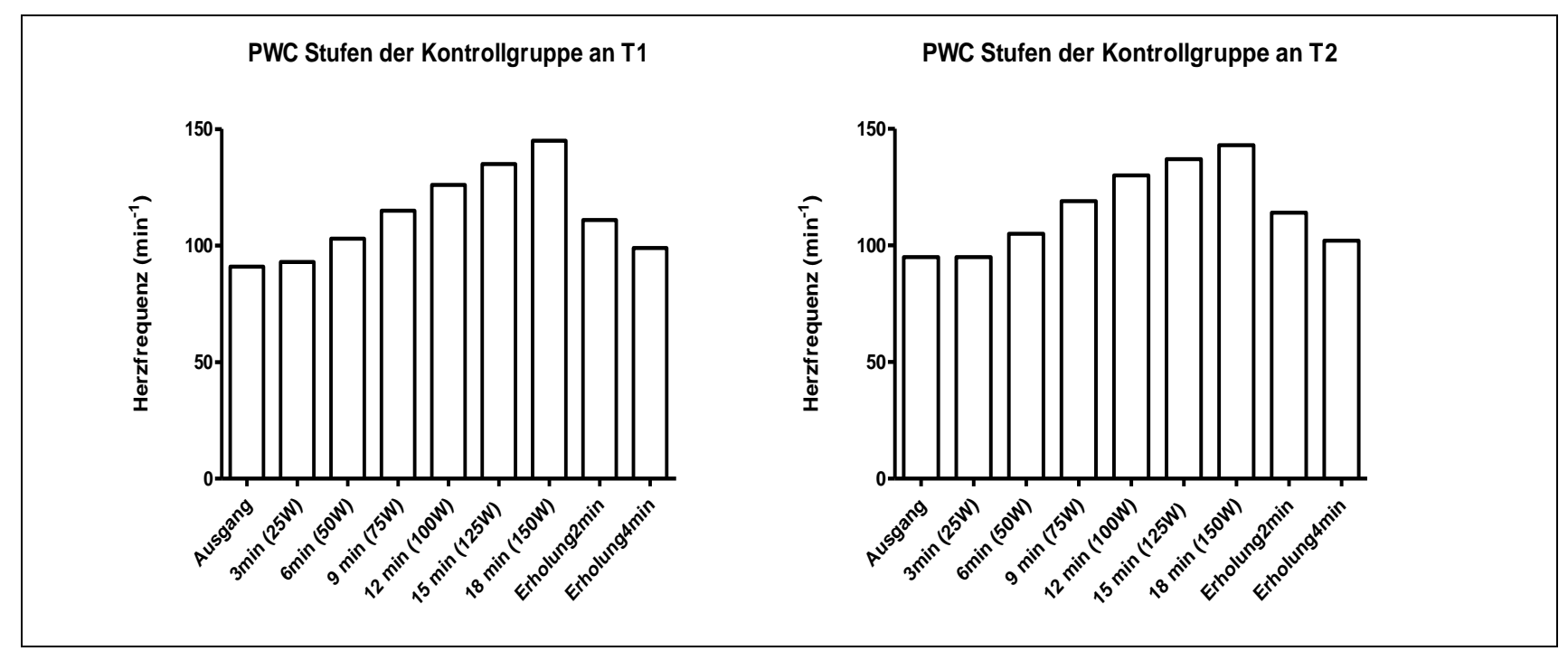

Abbildung 71 a und b: Pulsfrequenzen der Kontrollgruppe an den Messzeitpunkten T1und T2 während unterschiedlicher Wattstufen. 


\section{Diskussion}

Tumorbedingte Fatigue ist ein häufiges Symptom bei Krebsdiagnosen und unter bzw. nach der Krebstherapie. Es kann die Patienten auf den unterschiedlichsten Ebenen der psychosozialen und/oder physischen Funktionalität treffen und führt gewöhnlich zu einer deutlich wahrnehmbaren Verringerung der Lebensqualität (Jean-Pierre et al., 2007).

Die mittels Fragebögen erhobenen Daten der Fatigue, Lebensqualität, Angst und Depressionen zeigten, dass sportliche Aktivität während und nach der Rehabilitation von Brustkrebs einen positiven Einfluss auf diese Parameter nimmt. Entscheidend ist hierbei sicher auch die Intensität, mit welcher die Patientinnen das Trainingsprogramm durchgeführt haben und natürlich, welche Sportarten neben dem Training zusätzlich durchgeführt wurden. Hwang et al. (2008) zeigten in ihrer Studie, dass ein aerobes Training während der Radiotherapie bei Patientinnen nach diversen Brustkrebsoperationen zu einer Verbesserung der Lebensqualitätsparameter und der Fatigue führt. Grundsätzlich herrscht die Meinung vor, dass eher ein aerobes Ausdauertraining einen positiven Einfluss auf die Fatigue und andere Parameter der Krebserkrankung hat.

Durch die Resultate der Kraft- und Ausdauermessungen mittels Kraftmessdose und dem 6 Minuten Gehtest kann die erste Hypothese angenommen werden, da es zu einer Verbesserung der Kraft- und Konditionswerte kam. Die zweite Hypothese kann ebenfalls angenommen werden, da sich die Werte der Lebensqualitätsparameter, der Angst und Depressionen und die Werte der Fatigue in beiden Gruppen während der Rehabilitation verbessern, teils signifikant. Zu den weiteren Messzeitpunkten T3 und T4 verbessert sich die Trainingsgruppe weiterhin signifikant, die Kontrollgruppe jedoch nicht. Die dritte Hypothese kann teilweise angenommen werden, da die Dokumentation der Trainingsgruppenteilnehmerinnen darauf hinweist, dass durch das Trainingsprogramm ein selbstständiges trainieren angeregt wird. Es kann jedoch nicht gesagt werden, ob die Teilnehmerinnen der Kontrollgruppe ihr Sportprogramm wieder einstellen. Die vierte Hypothese kann wieder angenommen werden, da sich die subjektive Einschätzung der Leistungsfähigkeit der Teilnehmerinnen in der Trainingsgruppe signifikant gegenüber der Einschätzung der Kontrollgruppe verbessert hat.

In der Folgenden Diskussion sollen die Skalen und Subskalen der einzelnen Fragebögen aus Lebensqualität, Fatigue und Angst und Depressionen mit anderen Ergebnissen verglichen und diskutiert werden. Weiterer Bestandteil der Diskussion sind die Ergebnisse der 
physio- und sporttherapeutischen Fragebögen, sowie die Messergebnisse der Leistungsdiagnostiken.

\subsection{Diskussion der Instrumente}

Montazeri schrieb in seinem Literaturreview, dass unter anderem der EORTC QLQ-C30 Fragebogen zur Erhebung der Lebensqualität einer der am häufigsten eingesetzten und am besten entwickelten Fragebögen ist. Montazeri führte an, dass die Lebensqualität als einer der wichtigsten Endpunkte der klinischen Behandlung von Krebserkrankungen dargestellt wird (Montazeri, 2008). Daher sollte ein gut entwickelter Fragebogen zur Erhebung der Lebensqualität eingesetzt werden. Die Aussage, dass dieser Fragebogen zu denen am meisten genutzten Fragebögen zu Ermittlung der Lebensqualität gehört, wurde auch schon deutlich früher von Kemmler et al. getroffen (Kemmler et al., 1999). Im Bezug auf die chronische Fatigue sind die eindimensionalen Fragebögen wie der EORTC QLQC30 jedoch nur darauf focusiert, ob eine chronische Fatigue auftritt oder nicht (Jean-Pierre et al., 2007).

Der MFI-20 besteht aus fünf Skalen, die auf unterschiedliche Wahrnehmung der Fatigue basieren. "Generelle Fatigue" basiert auf generellen Aussagen über die „Funktionsfähigkeit" der Person, wie beispielsweise „Ich fühle mich erholt" (Smets et al., 1996).

Physische Fatigue bezieht sich auf ein körperliches Empfinden, welches mit dem Müdigkeitsempfinden in Verbindung steht. Reduzierte Aktivität und reduzierte Motivation sind über Parameter der „Antriebslosigkeit” abgedeckt. Die mentale Fatigue beinhaltet letztlich kognitive Symptome, wie etwa Konzentrationsstörungen. Jede Skala beinhaltet vier Posten, in denen die Patientin auf einer Sieben-Punkte-Skala angeben kann, inwiefern eine Aussage zutrifft.

Jean-Pierre et al., beschrieben den MFI als Instrument, welches sich auf die subjektive Erfahrung der Fatigue konzentriert. Die Skalen beurteilen generelle, mentale und physische Dimensionen der Fatigue, aber auch Ebenen der Motivation und Aktivitäten (Jean-Pierre et al., 2007).

Die initiale Version des MFI-20 wurde an Patienten mit chronischen Fatigue Syndrom getestet, sowie an Psychologiestudenten, Medizinstudenten, Wehrpflichtigen und jungen Medizinern. Hierbei wurde erwartet, dass Fatiguepatienten erschöpfter sind als Studenten; Soldaten sollten während eines intensiven Lehrgangs erschöpfter sein, als bei der 
Ruhephasen in der Kaserne. Junge Mediziner sollten ein höheres Maß an Erschöpfung während eines Lehrgangs der inneren Medizin haben, als vor dem Lehrgang (Smets et al., 1996). Smets et al. fassten zusammen, dass die Ergebnisse ihrere Studie die Validität des MFI-20 unterstützen (Smets et al., 1996). Auf Grund der steigenden Erfahrungswerte, dass es sich bei der chronischen Fatigue um ein multidimensionales Syndrom handelt, wurden auch multidimensionale Messinstrumente wie der MFI-20 entwickelt.

Laut Schwarz et al. sollte für jedes klinische Beispiel ein erwarteter Fatiguescore auf der Basis von Alter und Geschlechterverteilung berechnet werden. In einer repräsentativen Bevölkerungsstichprobe stellten Schwarz et al. in ihrer Studie an 2037 Personen im Alter von 14-92 Jahren über Grundwerte der Fatigue eine Korrelation mit Angst, Depressionen und der global quality-of-life-scale des EORTC QLQ-C30 fest (Schwarz et al., 2003).

Der Hospital Anxiety and Depression Scale (HADS) wird von Dolbeault et al. als "The established clinical case threshold" angeführt (Dolbeault et al., 2008). Kuhnt et al. beschrieben den HADS als ein valides und häufig eingestztes Instrument, um bei Tumorpatienten die Morbidität zu erfragen. Er besteht aus einer Angst-, und einer Depressionssubskala (Kuhnt et al., 2009).

\subsection{Die Messmethoden}

Die Kraftmessungen mit der digitalen Kraftmessdose von DIGIMAX stellte sich als gut durchführbar heraus. Wichtig war jedoch, den Patientinnen einige Probeversuche zu gewähren, um den erwarteten Messzyklus adäquat durchführen zu können. Hierbei handelte es sich um eine Eingewöhnung, um in späteren Messdurchgängen Übungseffekte und damit eventuell falsche Messwerte zu verhindern. So beschrieben es auch Heim et al. in ihrer Arbeit (Heim et al., 2007). Da sich der Messaufbau und die Messapparatur in der von Heim et al. angeführten Studie als gut erwiesen, wurde die Methode für diese Studie übernommen.

Der Sechs-Minuten-Gehtest ist in der rehabilitativen Physiotherapie ein häufig verwendeter Test (Wirz, 2006). Das Vorgehen sollte allerdings standardisiert sein und in dieser Form bei jedem Test wiederholt werden. Generell wird der Test bei Patienten mit kardiovaskulären oder pulmonalen Erkrankungen eingesetzt, um deren Belastbarkeit zu überprüfen (Steffen et al., 2002). Der Test sollte auf Grund seiner Einfachheit in der Anwendung und seiner Nähe zur täglichen Aktivität der Patientinnen in dieser Studie 
genutzt werden. Des Weiteren ist es für die Patientinnen auch ein optimaler Test für die Zeit nach der Rehabilitation, um die aerobe Ausdauerleistungsfähigkeit bestimmen und weitere Änderungen dokumentieren zu können. Außerdem konnte in diversen Studien eine gute Test-Retest Reliabilität ermittelt werden (Steffen et al., 2002).

Das Leistungskriterium Physical Working Capacity (PWC) wurde Ende der 40er Jahre von Wahlund eingeführt. Man bezog die Physical Work Capacity auf eine Pulsfrequenz von 170/min bei Arbeit auf dem Fahrradergometer bei jungen gesunden Menschen und Sportlern (Hollmann et al., 2006). Der Test kann jedoch auch mit niedrigeren Zielpulsfrequenzen $(110,130,150)$ durchgeführt werden. Diese stehen in Abhängigkeit von Alter, Geschlecht und Gesundheitszustand der Testperson. Die Auswertung des PWC stellte sich als schwierig heraus, da eine Querschnittmittelwertrechnung hier nicht durchführtbar war. Das Ziel des Tests war es, eine individuelle Leistungsbeurteilung zum Messzeitpunkt T1 abgeben und zum Messzeitpunkt T2 eventuelle Veränderungen der körperlichen Leistungsfähigkeit darstellen zu können. Da aber Patientinnen aus beiden Studiengruppen durch eine Verordnung in der Klinik Fahrradergometertraining durchgeführt haben könnten, ist eine gruppenweise Auswertung nicht repräsentativ, sondern nur eine Auswertung der einzelnen Patientinnen einer Gruppe wäre repräsentativ. Es werden in dieser Arbeit die Mittelwerte der Herzfrequenz während der einzelnen Belastungsstufen verglichen, wobei hier berücksichtigt werden muss, dass sich die Patientinnenzahl mit andauernder Testzeit ständig verringerte, da die Belastungsstufen die Patientinnen irgendwann an den Zielpuls brachten oder aber zur Aufgabe zwangen. Die geringen Unterschiede sind auf das oben schon angesprochene, eventuell ähnliche Trainingsprogramm der beiden Gruppen während der Rehabilitation zurückzuführen.

\subsection{Die Patientenrekrutierung und der Drop-out}

Ein frühzeitiges Problem ergab sich bereit während der Patientenrekrutierung. Durch die wochenweise randomisierte Rekrutierung kamen Patientinnen der Trainings- und Kontrollgruppe zwangsläufig in Kontakt und tauschten sich über die Studie aus. Patientinnen der Kontrollgruppe stellten sich nun die Frage, warum sie kein Übungsprogramm erhalten haben und zogen schon zum ersten Messtermin oder sogar vorher ihre Teilnahmebereitschaft zurück, da sie keinen persönlichen Nutzen durch eine Studienteilnahme hatten. Dadurch bildete sich schon ein geringerer Ausgangswert in der 
Kontrollgruppe zum Messzeitpunkt T1. Zum Messzeitpunkt T2 zeigte sich mit 89,3\% dann jedoch eine höhere Teilnahmebereitschaft in der Kontrollgruppe, als mit $68,6 \%$ in der Trainingsgruppe. Dies kann darauf zurückgeführt werden, dass die Teilnehmerinnen der Trainingsgruppe die erhöhte Anforderung durch das zusätzliche Übungsprogramm in Kombination mit dem Rehabilitationsprogramm der Klinik nicht erfüllen konnten oder sich diesbezüglich überfordert fühlten. Zum Messzeitpunkt T3 lag der Rücklauf der Fragebögen in der Trainingsgruppe bei 58,6\%, also 10\% niedriger als zu T2. In der Kontrollgruppe lag die drop-out Rate von T2 nach T3 bei etwa 18\%, weitere knapp 11\% der Teilnehmerinnen sendeten die Frägebögen an T4 nicht zurück. Der massive drop-out von T2 bis T4 von knapp 30\% könnte darauf zurückzuführen sein, dass die Teilnehmerinnen der Kontrollgruppe keine wirkliche Bindung zu der Studie aufbauen konnten und daher nach mehreren Monaten keine Relevanz mehr sahen, weiterhin an der Studie teilzunehmen. Eine eindeutig engere Bindung zu der Studie zeigten die Teilnehmerinnen der Trainingsgruppe, da die drop-out Rate vom Messzeitpunkt T2 zu T3 bei nur 10\% lag und von T3 zu T4 bei nur 1,5\%. Durch die erhöhte drop-out Rate in der Trainingsgruppe von T1 zu T2 und der relativ gleichmäßig abnehmenden Teilnehmerzahl in der Kontrollgruppe von T1 bis T4 liegen die Teinnehmerzahlen am Ende der Studie mit 57,1\% in der Trainingsgruppe und $60,7 \%$ in der Kontrollgruppe wieder relativ gleichmäßig beieinander.

\subsection{Die physiotherapeutischen Ergebnisse}

Zwei Drittel aller befragten Patientinnen gaben zu den Messzeitpunkten T1 und T2 an, keine schmerzhaften Operationsnarben beibehalten zu haben, etwa ein Drittel der Patientinnen hatte noch Schmerzen im Bereich des operativen Eingriffs. Ebenso gab eine Mehrheit (etwa drei viertel) der Patientinnen an keine Schwellungen im Operationsbereich oder dem Arm zu haben, ein Viertel der befragten Patientinnen gab an, Schwellungen im Operationsbereich und/oder des Armes zu haben. Die relativ geringe Anzahl von Problemen in den beiden Bereichen ist darauf zurückzuführen, dass die Operationen bei den meisten Patientinnen schon einige Wochen oder sogar Monate zurücklagen und es sich bei Beschwerden an den Narben oder Ödemen an den Extremitäten (hier besonders der Arme und Handgelenke) eher um akute postoperative Probleme handelt. Weiterhin kommt hinzu, dass noch vor zehn Jahren die axilläre Ausräumung (Axillary dissection) als Standardoperationsmethode im Management von Brustkrebs durchgeführt wurde (Kuehn et al., 2000). Heutzutage hat sich die sentinel lymph node Biopsie (SLN) als 
Standardmethode bewährt. Ihre Durchführbarkeit und Genauigkeit wird in der Literatur weitreichend beschrieben (Port et al., 2010). Daher wäre auch der deutlich minimalere und schonendere Eingriff eine Erklärung, warum die Patientinnen nicht so stark unter postoperativen Beschwerden zu leiden hatten.

Beide Gruppen gaben zu T1 und T2 an, unter verspannter (hypertoner) Schulter-NackenMuskulatur zu leiden (80\% der Trainingsgruppe und 70\% der Kontrollgruppe). Interessanterweise reduzierte sich die Anzahl derer mit Verspannungen in der Trainingsgruppe an T3 auf $65 \%$ und an T4 auf 55\%, wogegen es keine Veränderungen in der Kontrollgruppe über den Zeitraum von 6 Monaten gab. Wingate untersuchte die Effektivität von Physiotherapie bei Patientinnen nach Brustkrebsbehandlung und stellte fest, dass eine Therpie nach Mastektomie eine bessere Funktionalität ergab (Wingate, 1985).

Gosselink et al. bestätigten diese Aussage 2003 und fassten zusammen, dass Physiotherapie nach Brustoperationen dringend nötig ist, um die Funktionen der oberen Extremität wieder adäquat herstellen zu können (Gosselink et al., 2003). McNeely et al. beschrieben in ihrem Literaturreview über Trainingstherapie bei Dysfunktionen der oberen Extremität, dass sowohl Frühmobilisation postoperativ, als auch ein strukturiertes Übungsprogramm die Beweglichkeit im Schultergelenk signifikant verbessern kann (McNeely et al., 2010).

Da auch die Übungen aus dem getesteten Übungsprogramm in der Physiotherapie zur Kräftigung und Mobilisation des Schulter-Nacken-Komplexes ihre Anwendung finden, kann geschlussfolgert werden, dass die Durchführung des Übungsprogramms in der Trainingsgruppe einen positiven Einfluss auf die verspannte Muskulatur hatte. Des Weiteren sind muskuläre Verspannungen häufige Begleiterscheinungen bei Depressionen. Blumenthal et al. beschrieben in ihrer Arbeit über ältere Patienten mit Depressionen, dass sportliche Aktivität eine Alternative zu Antidepressiva bei der Behandlung sein könnte (Blumenthal et al., 1999).

Der positive Einfluss des Übungsprogramms zeigt sich auch bei den Bewegungseinschränkungen und Einschränkungen im täglichen Leben. Hier zeigte die Trainingsgruppe von T1 nach T4 eine Verbesserung von 17\%, die Kontrollgruppe verschlechterte sich, nach minimaler Verbesserung an T4 von 4\%, drei Monate später zum Messzeitpunkt T4 um 12\%. Hier ist eindeutig zu sehen, dass ein regelmäßiges Training die täglichen Aktivitäten positiv beeinflussen kann. Dies bestätigten auch Springer et al. in ihrer Studie. Sie zeigten, dass Patientinnen nach Brustkrebsoperation, die eine 
anschließende physiotherapeutische Intervention bekamen, schon nach drei Monaten ihre Basiswerte im Bewegungsausmaß und in der Kraft wieder erlangten (Springer et al., 2010).

\subsubsection{Die körperliche Leistungsfähigkeit}

Besonders bei den Patientinnen der Trainingsgruppe änderte sich die subjektiv empfundene körperliche Leistungsfähigkeit sehr deutlich. Von dem Messzeitpunkt T1 nach T4 änderte sich in der Trainingsgruppe die körperlich eingeschätzte Leistungsfähigkeit von 40\% mit mittelmäßiger Einschätzung auf 53\% gut eingeschätzter Leistungsfähigkeit. Die Kontrollgruppe änderte ihre Einschätzung von T1 nach T4 nicht, sie stand zu beiden Messzeitpunkten bei etwa $50 \%$ mittelmäßiger subjektiv empfundener körperlicher Leistungsfähigkeit. Dies zeigt, dass das strukturierte Übungsprogramm dazu beiträgt, die subjektiv empfundene, körperliche Leistungsfähigkeit zu verbessern. Sprod et al., konnten in ihrer Studie über die Auswirkungen eines drei- bzw. sechsmonatigen Ausdauertrainings auf die Physis und die Psyche von Brauskrebspatientinnen zeigen, dass ein individuell verordnetes Übungsprogramm über einen Zeitraum von sechs Monaten bereits nach drei Monaten regelmäßigen Trainings die herzkreislaufbedingte Ausdauer, die Fatigue und Anzeichen von Depressionen verbessert. Nach sechs Monaten gab es additive Effekte auf die Lungenfunktion und die muskuläre Ausdauer (Sprod et al., 2010). Die Ergebnisse der vorliegenden Studie bestätigen die Ergebnisse von Sprod et al., insbesondere im Bezug auf die Fatigue und die Depressionen, welche sich in der vorliegenden Studie ebenfalls nach drei Monaten signifikant verbessert hatten. Dies kann besonders für FatiguePatientinnen im Bezug auf das tägliche Leben von entscheidender Bedeutung sein.

\subsection{Die psychoonkologischen Ergebnisse}

In den letzten Jahren wurden die Einflüsse von sportlicher Belastung auf die Lebensqualität, die Fatigue und Ängste und Depressionen mehr und mehr Bestandteil von Studien bevor, während und nach der Krebstherapie.

Noch immer sind einiger Mediziner der Ansicht, dass aerobes, körperliches Ausdauertraining Krebspatienten eher schaden, statt helfen könnte. Neben den bekannten positiven Einflüssen von sportlicher Aktivität auf das physische und psychische Wohlbefinden, belegen immer mehr Studien, dass ein strukturell gutes Übungsprogramm nicht zu einer Überforderung, sondern zu einer Besserung vieler Begleitsymptome der 
Tumortherapie führt. Strömbeck et al. konnten in ihrer Studie an rheumatisch erkrankten Frauen zeigen, dass sich aerobes Training positiv auf die rheumatisch bedingte Fatigue und Depressionen auswirkt (Strömbeck et al., 2007).

\subsubsection{Die Lebensqualität (Quality of Life)}

Bei der Auswertung des Scorings des EORTC-QLQ C-30 ist zu beachten, dass ein Unterschied in der Berechnung der Werte in den Funktionsskalen zu den Symptomskalen besteht. Dies hat zur Folge, dass in den Symptomskalen höhere Scorings negativ zu bewerten sind, in den Funktionsskalen jedoch bedeuten höhere Scorings bessere Ergebnisse.

Die Fatigue fällt unter die Symptomskalen. Dieser Wert änderte sich positiv signifikant in der Trainingsgruppe. Auch in der Kontrollgruppe gab es eine Besserung, diese stagnierte jedoch ab dem Messzeitpunkt T2. Wiggins et al. konnten in ihrer Studie anhand einer zwölf- monatigen Trainingsintervention nachweisen, dass sich das psychologische Wohlbefinden vom Basiswert bis neun Monate nach Trainingsbeginn verbessert (Wiggins \& Simonavice, 2008). Auch Valenti et al. beschrieben in ihrer Arbeit die starke Korrelation zwischen anstrengenden Übungen und der Lebensqualität (Valenti et al., 2008). Dies bestätigt die Hypothese der vorliegenden Studie, dass die Werte der Lebensqualität und der Fatigue sich in der Trainingsgruppe auch nach der stationären Rehabilitation weiterhin verbessern werden. Keine oder nur minimale Verbesserungen wurden in der Kontrollgruppe erwartet.

Die Kontrollgruppe zeigte einen ähnlichen Verlauf wie die Patientinnen in einer Studie von Montazeri et al., welche 18 Monate nach der eigentlichen Tumorbehandlung immer noch über globale Probleme der Lebensqualität klagten, sich sogar die meisten Parameter der Symptomskalen nicht verbesserten (Montazeriet al., 2008).

Diverse Studien haben gezeigt, dass auch die Kognition oder das "Cognitive Functioning“ mit der Tumortherapie, hier besonders mit der angewanden Chemotherapie, korrelliert und dass es signifikante Unterschiede, beispielsweise bei den Scores des Emotional Functioning zwischen Burstkrebspatientinnen während der angewanten Chemotherapie und Gesunden gibt (Brezden et al., 2000). Hierzu nutzten sie den High Sensitive Cognitive Screen (HSCS). Dieser Test ist sensitiv für kognitive Beeinträchtigungen. Als weiteres Instrument wurde der Profile of Mood States (POMS) genutzt, welcher sehr sensitiv auf Gemütsveränderungen wie Ängste und Depressionen reagiert. 
In der vorliegenden Studie zeigte sich ein Zusammenhang von körperlicher Aktivität und, sowohl dem Emotional als auch dem Cognitive Functioning. Die Subskala des Cognitive Functioning des EORTC in der vorliegenden Studie zeigten in beiden Gruppen (Trainingsund Kontrollgruppe) eine Besserung der Kognition von T1 nach T2, in der Trainingsgruppe sogar signifikant. Dieser Unterschied ist auf die höheren Ausgangswerte an T1 in der Kontrollgruppe zurückzuführen. Die weiterhin signifikanten Verbesserungen der Kognition in der Trainingsgruppe von T2 bis T4 zeigen, dass eine regelmäßige sportliche Betätigung auch einen Einfluss auf die kognitiven Fähigkeiten hat.

Dies belegen auch Korstjens et al. in ihrer Studie. Sie zeigten, dass nach einem zwölfwöchigen Rehabilitationsprogramm signifikante Verbesserungen in der globalen Lebensqualität, der emotionalen und auch der kognitiven Funktion eintreten (Krostjens et al., 2006).

In der vorliegenden Studie verbesserte sich der Physical Functioning Score der Trainingsgruppe von T1 bis T3 und T1 bis T4 jeweils signifikant. In der Kontrollgruppe gab es ebenfalls eine signifikante Besserung vom Messzeitpunkt T1 nach T3, drei Monate später, am Messzeitpunkt T4 war der Physical Functioning Score jedoch wieder gesunken. Dieses Ergebnis läßt die Schlussfolgerung zu, dass ein weiter durchgeführtes Training die Physis auch nachhaltig beeinflusst und eine positive Wirkung auf die subjektiv wahrgenommene körperliche Leistungsfähigkeit hat. Diese Aussage fassten auch Schmitz et al. zusammen, indem sie beschrieben, dass Übungsprogramme während und nach der Tumorbehandlung sicher sind und zu Verbesserungen tumorassozierter Fatigue führen. Der Ratschlag „verhindere Inaktivität" wird sogar bei Tumorpatienten mit bestehender Erkrankung und schwierigen Behandlungen als hilfreich angesehen (Schmitz et al., 2010). Allerdings gibt es auch bestimmte Situationen, in denen körperliche Aktivität kontraindiziert ist. Daher sollte vor der Erstellung eines Rehabilitations- oder Trainingsplans eine sportmedizinische Untersuchung durchgeführt werden, um eventuelle Risiken auszuschliessen.

\subsubsection{Die fünf Dimensionen des MFI}

Die fünf Dimensionen des Multidimensional Fatigue Inventory (MFI) bestehen aus der allgemeinen, der physischen und der mentalen Fatigue, der reduzierten Motivation und der reduzierten Aktivität. 
Das sportliche Aktivität anhand eines strukturierten Übungsprogramms auf alle genannten fünf Dimensionen einen positiven Einfluss hat, zeigen die Ergebnisse der vorliegenden Studie. Somit findet sich eine weitere Hypothese bestätigt, nämlich das sich die Messwerte der Trainingsgruppe durch die Durchführung das Übungsprogramms auch nach der Rehabilitation weiterhin verbessern. Die signifikanten Verbesserungen der Trainingsgruppe in den Dimensionen allgemeine, mentale und physische Fatigue über den gesamten Trainings- und Testzeitraum von sechs Monaten bestätigen die Aussagen über positive Einflüsse sportlicher Aktivität auf das physische und psychische Wohlbefinden. Hier darf der Rehabilitationseffekt nicht außer Acht gelassen werden. Heim et al. beschrieben in ihrer Arbeit über die Effekte eines stationären Rehabilitationsprogramms auf die gesundheitsbezogende Lebensqualität, dass sich am Ende eines komplexen Rehabilitationsprogramms die gesundheitsbezogene Lebensqualität in vielen Beriechen verbesserte und empfahlen sogar eine fortführende ambulante Therapie, um die Rehabilitationserfolge aufrecht zu erhalten (Heim et al., 2001). Die Ergebnisse der vorliegenden Studie bestätigen die Aussage von Heim et al., die einen anhaltenden Effekt der stationären Rehabilitation auch nach drei Monaten entdeckten. Dieser wird besonders in der Kontrollgruppe dieser Studie deutlich, da auch hier eine positive Entwicklung der Lebensqualität und der Fatigue von T1 nach T2, also während der stationären Rehabilitation zu verzeichnen ist. Sowohl die körperliche Aktivität, als auch die psychosoziale Unterstützung scheinen hier eine wesentliche Rolle zu spielen. Terson de Palleville et al. konnten in ihrer Studie zeigen, dass die Fatigue gesenkt und die Aktivitäten des täglichen Lebens durch ein aerobes Trainingsprogramm eine Woche vor der Chemotherapie startend und dann weiterhin während der Behandlung durchgeführt, gesteigert werden können (Terson de Palleville et al., 2007). Auch Cramp et al. beschrieben in einem Literaturreview, welches 28 Studien beinhaltete, die sich mit Training und Tumorerkrankung/Tumortherapie beschäftigten, dass körperliches Training statistisch signifikantere Unterschiede in der Fatigue zeigten, als die Kontrollinterventionen, egal ob das Training während oder nach der Tumorbehandlung durchgeführt wurde (Cramp \& Daniel, 2008). Die signifikanten Verbesserungen in den Subskalen des MFI der vorliegenden Studie, und hier besonders die reduzierte Aktivität und die Verbesserung der Motivation (besserer Scores der reduzierten Motivation im MFI) zeigen, dass es durch körperliche Aktivität zu einer positiven Beeinflussung der Fatigue kommt. Gleiches führten auch Dimeo et al. in ihrer Studie über aeorbes Training unmittelbar nach der hochdosierten Chemotherapie an, in der sie untersuchten, dass 
aerobes Training nach Chemotherapie sicher durchgeführt werden und teilweise den Verlust der physischen Aktivität verhindert (Dimeo et al.,1997). Van Weert et al. zeigten, dass physisches Training in Kombination mit kognitiver Verhaltenstherapie und physischem Training allein im Vergleich mit keiner Intervention positive Effekte auf die Fatigue hatten (Van Weert et al., 2010).

Die weiteren Subskalen, reduzierte Aktivität und reduzierte Motivation des MFI veränderten sich in der Trainingsgruppe ebenfalls positiv signifikant vom Messzeitpunkt T1 zu T2 sowie von T2 zu T3 und T4. In der Kontrollgruppe hingegen blieben signifikante Veränderungen aus, in der Dimension der reduzierten Motivation gab es sogar eine Verschlechterung der Werte von T2 zu T4. Diese Ergebnisse verdeutlichen, welchen Einfluss besonders die Motivation auf die übrigen Parameter wie Aktivität, mentale und physische Fatigue haben kann. Laut Kuhnt et al. hat insbesondere der soziale Beistand einen Einfluss auf die reduzierte Motivation (Kuhnt et al., 2009). Des Weiteren gaben Kuhnt et al. in ihrer Studie an, das Depressionen die stärkste Wirkungsvariable in allen Fatiguedimensionen waren. Reuter et al. fanden dies bereits in ihrer Studie über die Verbindungen von Bewältigungsstrategien und Schmerz, Alter, Depressionen und der Fatigue heraus (Reuter et al., 2006).

\subsection{3 Ängste und Depressionen (HADS)}

Der HADS wurde speziell für medizinisch kranke Patienten entwickelt. Er schließt somatische Symptome von Depressionen aus und verhindert dadurch Symptomüberschneidungen zwischen somatischen Erkrankungen und Gemütserkrankungen (Reuter et al., 2006).

Die Sensitivität des HADS für Depressionen liegt laut Alexander et al. bei $50 \%$, die Spezifität bei $97 \%$. Für die Ängste lag die Sensitivität bei $71 \%$, die Spezifität bei $86 \%$ (Alexander et al., 2009). Das strukturierte klinische Interview wurde an 200 überlebenden Brustkrebspatientinnen durchgeführt, um das Leiden unter Ängsten und Depressionen zu indentifizieren. Die Werte machen den HADS zu einem sehr wirkungsvollen Selfscreening Instrument, um Ängste und Depressionen bei Patienten festzustellen (Alexander, Palmer, \& Stone, 2009).

In den Gruppen der vorliegenden Studie konnte der HADS daher auch als valides Screeninginstrument eingesetzt werden. In der Trainingsgruppe verbesserte sich der Angstscore signifikant von T1 durchgehend bis T4. In der Kontrollgruppe gab es keine 
signifikanten Verbesserungen. Ebenfalls signifikante Besserungen gab es im Depressionsscore der Trainingsgruppe von T1 zu T3 und T4. In der Kontrollgruppe hingegen kam es zu einer minimalen Besserung von $T 1$ zu T2, jedoch zu einer Verschlechterung von T2 bis T4. Dies läßt die Schlußfolgerung zu, dass die fehlende sportliche Aktivität der Kontrollgruppe einen Einfluss auf die Angst- und Depressionswerte hat. Daley schlussfolgerte, dass sportlich aktive Menschen wichtige Vorteile in der mentalen Gesundheit, insbesondere bei Depressionen, haben (Daley, 2008). Sportliche Aktivität im Zusammenhang mit der Behandlung von Depressionen ist schon seit Jahrzehnten ein wichtiges Thema in der Forschung. Die Ergebnisse dieser Studie bestätigen, dass sportliche Aktivität einen positiven Einfluss auf Depressionen hat. Es wird deutlich, dass die körperliche Aktivität auf viele kognitive Bereiche einen positiven Einfluss hat, da sich in der vorliegenden Studie außer den Depressionen auch die mentale Fatigue und das Cognitve Functioning verbessern.

Jedoch sollten die Ergebnisse nicht falsch interpretiert und die sportliche Aktivität dabei als „Allheilmittel“ bei Depressionen beschrieben werden. Daley, 2008 schrieb in der Zusammenfasung ihrer Studie ebenfalls, dass die Autoren von Reviews und Metaanalysen davor warnen, die Ergebnisse ihrer Studie als „Normwerte“ zu nutzen. Neben den Bedenken über die Methoden und der Einsetzbarkeit sportlicher Aktivität haben Reviews allerdings auch gezeigt, dass sportliche Aktivität als Behandlung von Depressionen effektiver ist, als gar keine Behandlung (Daley, 2008). Auch andere Faktoren, wie erhöhte Selbsterfahrung oder Ablenkung durch die Teilnahme an sportlichen Aktivitäten, können dafür verantwortlich sein, dass sich die Symptome der Depressionen verbessern (Nabkarson et al., 2005). Zusätzlich fanden Nabkarson et al. eine Senkung des 24Stunden Cortisol im Urin und eine Verringerung in der Sekretion von Epinephrine nach dem Sportprogramm. Die Konzentration von Epinephrine im Urin wurde als Indikator für sympathische Nervenaktivität bei der Untersuchung von psychophysischem Stress und Depressionen genutzt (Nabkarson et al., 2005). Duclos et al. schrieben, dass es als Folge von physischer Aktivität zu einer Reduktion des Stresshormons Cortisol kommen kann. Die vielfältigen und weitreichenden Folgen körperlicher Aktivität auf psychologischen Stress und Depressionen sind somit bekannt, ihre Wirkungsweise jedoch noch nicht vollends geklärt (Duclos et al., 2003). 


\subsection{Einfluss von psychischem und physischem Stress auf die Immunologie}

Diverse Studienergebnisse haben gezeigt, dass psychologischer Stress zu einer Verringerung der Immunaktivität und der natürlichen Killerzellen (NK-Zellen) führt (Esterling et al., 1994). In einer Studie von Witek-Janusek et al. wurde beschrieben, dass eine Brustbiopsie bei Patientinnen mit Verdacht auf Brustkrebs ein emotionales Erlebniss darstellt, welches durch vermehrten Stress, Angst und Gemütsveränderungen charakterisiert ist. Diese emotionale Erfahrung wird begleitet von einer reduzierten NkZellenaktivität und einer Zytokindysregulation (Witek-Janusek et al., 2007). Tatsächlich zeigten auch Brenu et al. einen Zusammenhang zwischen chronischer Fatigue und einer herabgesetzten zytotoxischen Aktivität der NK-Zellen (Brenu et al., 2010). Es wird jedoch über die sogenannte sportbedingte Zellapoptose (Zelluntergang) der Lymphozyten gesprochen. Hierbei müssen extreme Werte (75\%-85\%) der aeroben Kapazität unter sehr anstrengender sportlicher Belastung erreicht werden (Navalta et al., 2010).

Mooren et al. haben festgestellt, dass gut trainierte Individuen eine geringere Lymphozytenapoptosis erfahren, als gleiche Individuen mit einem schlechteren Trainingsstatus (Mooren et al., 2010). Hierzu schrieben Hsu et al., dass drei aufeinander folgende Tage von moderater bis zu hochintensiver Anstrengung als Frequenz nötig sind, um eine sportinduzierte Lymphozytenapoptose zu entwickeln (Hsu et al., 2010). Ein Review über Sport und Krebsrehabilitation von Spence et al. in dem zehn Studien eingeschlossen waren zeigten, dass die meisten Interventionen aus aerobem Training und Krafttrainingsprogrammen bestanden. Alle Studien zeigten eine Verbesserung der Kraft, Lebensqualität, Fatigue und Immunfunktionen, sowie einen Anstieg der Haemoglobinkonzentration (Spence et al., 2010). Gleiches ergab die vorliegende Arbeit im Bezug auf die Lebensqualität, die Fatigue und die Kraftwerte.

\subsection{Das Übungsprogramm}

Die UK National Institute of Clinical Excellence (NICE) Guideline für Depressionen rät zu einem drei mal, wöchentlichen strukturierten, beaufsichtigten Übungsprogrammen (45 Min. bis 60 Min.), über einen Zeitraum von zehn bis zwölf Wochen bei milden Depressionen (NICE, 2007).

Spence et al. fanden in ihrem systematischen Review zehn Studien, in denen Brustkrebspatientinnen mit Fatigue untersucht wurden. Sie entdeckten, dass die meisten 
Interventionen in den Studien aerobe Übungsprogramme oder Krafttraining beinhalteten und diese positive Einflüsse auf physische Funktionen, Lebensqualität und Fatigue hatten (Spence et al., 2010). Ein gruppenbasierendes Übungsprogramm konnte soziale Beziehungen der Patienten fördern, die Motivation anheben und so für eine relativ hohe Teilnahme sorgen (Nabkarson et al., 2005). Die Durchführung von selbstständigem Training kann sowohl Vorteile als auch Nachteile mit sich bringen. Die Kontrolle und damit das richtige Ausführen der Übungen ist beim individuellen Training deutlich erschwert und kann so besonders für Patienten, deren Körpergefühl nicht aussreichend ist, gefährlich sein. Der motivationelle Aspekt spielt aber sicherlich die größte Rolle und birgt die meisten Probleme. Sich alleine zu Hause zum Sport zu motivieren, fällt den meisten Patienten extrem schwer. Das häusliche Umfeld wird nicht als Sportstätte betrachtet, und es fällt immer leichter, sportliche Aktivitäten in den dafür vorgesehenen Einrichtungen durchzuführen. Zusätzlich dazu könnten sich sportlich unerfahrene Patienten entweder über- oder unterfordern. Allerdings ist betreute Selbsthilfe (guided self help) mehr, als Patienten nur Literatur zum Lesen mitzugeben. Oft basiert sie auf kognitiver und verhaltenstechnischer Herangehensweise (NICE, 2007).

Der Tatsache zufolge, dass die Teilnehmerinnen der Trainingsgruppe der vorliegenden Studie sowohl die Kraft- als auch die Dehnübung mit über 90\%iger Regelmäßigkeit durchgeführt haben, spricht für die Einsetzbarkeit des Übungsprogramms „Fitness trotz Fatigue", aber auch für die korrekt Einweisung der Patientinnen und die dadurch bedingte Herangehensweise durch die Patientinnen. Ein Ausdauertraining wurde von den Patientinnen durchschnittlich 5-7 Stunden pro Monat durchgeführt. Hierbei unterschieden sich jedoch die Art, Intensitäten und Umfänge der durchgeführten Sportarten erheblich. In der Pilotstudie von Elsner von der Malsburg trainierten die Teilnehmerinnen der Studie nur $37 \%$ der Kraft- und 42\% der Dehnübungen. Dafür wurde das Ausdauertraining in Form von Nordic Walking weit über $100 \%(100 \%=2 \times$ wöchentlich) trainiert (Elsner von der Malsburg, 2006). Ein Grund für diese deutlichen Unterschiede könnte sein, dass in der vorliegenden Studie das Hauptaugenmerk auf die Kraft- und Dehnübungen gelegt wurde, das Ausdauertraining konnte von den Patientinnen frei gewählt werden. Es war also nicht ausschlaggebend, ob die Patientinnen Schwimmen, Jogging, Nordic Walking o.ä. als Ausdauersport durchgeführt haben. Des Weiteren war es vielleicht zu viel an sportlichem und auch zeitlichem Einsatz für die Patientinnen, jeweils $2 x$ wöchentlich das Übungsprogramm und zweimal wöchentlich eine Ausdauersportart durchzuführen. 


\subsection{Kraftmessung und Ausdauertests}

Es zeigten sich in beiden Gruppen ein zeitlicher Effekt im Bereich der isometrischen Maximalkraft von T1 zu T2. Das bedeutet, dass die dreiwöchige Rehabilitation und die währenddessen durchgeführten Sport- und Bewegungstherapien sowohl in der Trainingsals auch in der Kontrollgruppe einen positiven Einfluss auf die Kraft hatten. Denn sowohl die Trainings- als auch die Kontrollgruppe führten verschiedene bewegungs- und sporttherapeutische Maßnahmen als Teil der stationären Rehabilitation durch. Der Kraftzuwachs ist auf neuronale Anpassungen zurüchzuführen. Durch regelmäßig durchgeführtes Krafttraining findet zunächst eine neuronale Anpassung statt, d.h. bei Muskelarbeit werden gleichzeitig mehrere Muskelfasern rekrutiert und diese werden mit zahlreichen Nervenimpulsen versorgt. Durch diese koordinative Anpassung (verbessertes Zusammenspiel von ZNS und Muskulatur) arbeiten die Muskelfasern innerhalb eines Muskels (intramuskulär) und die Muskelfasern verschiedener Muskeln (intermuskulär) besser zusammen. Diese Verbesserung der intra- und intermuskulären Koordination sorgt für eine Kraftzunahme und einer verbesserten Muskelökonomie.

Ein weiterer wichtiger Punkt des Krafttrainings ist eine Steigerung der täglichen Leistungsfähigkeit und somit auch der Lebensqualität. Courneya et al. fassten zusammen, dass ein Krafttrainingsprogramm sogar während der Chemotherapie die Selbsteinschätzung, die physische Fitness, die Körperkomposition und auch die Beendigungsrate der Chemotherapie bei Brustkrebspatientinnen verbessert und erhöht. Die Autoren randomisierten 242 Brustkrebspatientinnen in eine betreute Krafttrainingsgruppe und eine aerobe Ausdauergruppe (Courneya et al., 2007).

Der 6 Min. Gehtest ist ein Test zur Bestimmung der funktionellen Performance, speziell der Gangausdauerfähigkeit (Foley et al., 2010). Zudem ist der Test leicht durchführbar, kostengünstig und sicher. Er enspricht viel mehr den täglichen Beanspruchungsmustern der Patienten. Daten anderer Studien belegen, dass der Test eine gute Reproduzierbarkeit besitzt (Guyatt et al., 1985).

Die in der Kontrollgruppe sogar signifikant längeren Wegstrecken während des 6 Min. Geh-tests zeigen eindeutig, dass das Rehabilitationsprogramm der Klinik auch die physische Ausdauerleistungsfähigkeit verbessert. Auch hier muß der Zeiteffekt genauer betrachtet werden. Die Rehabilitationsdauer von ca. drei Wochen wird nicht aussreichend sein, um die aerobe Ausdauerleistungsfähigkeit der Patientinnen so deutich zu 
verbessern. Auch hier wird das strukturierte Trainingsprogramm der Klinik insbesondere die Selbsteinschätzungsfähigkeit der Patientinnen und die allgemeine körperliche Belastbarkeit verbessert haben. Erwähnt werden sollte an dieser Stelle, dass durch Training erzielbare Leistungssteigerungen vom Trainingspensum, d.h. von der Trainingsintensität und Trainingsdauer, abhängig sind (Schmidt \& Thews, 1987). Diesbezüglich ist es wichtig, welches Trainingspensum die Teilnehmerinnen der Studie besonders im Ausdauertrainingsbereich während der Rehabilitation durchgeführt haben. Da dies nicht mehr nachzuvollziehen ist, soll hier die Erklärung reichen, dass die Leistung bei konstantem Trainingspensum zu Beginn einer Trainingsperiode erheblich zunimmt, je länger jedoch die Trainingsperiode dauert, der Leistungszuwachs geringer wird (Schmidt \& Thews, 1987).

Die hohe Trainingsbeteiligung von ca. $90 \%$ läßt auf Grund der vorher genannten Kriterien die Schlussfolgerung zu, dass durch die Einstellbarkeit des Übungsprogramms auf die aktuelle Leistungsfähigkeit der Patientinnen das „optimale" Trainingspensum erreicht werden konnte.

Eine weitere wichtige Erkenntnis konnte nach Beendigung der Studie getroffen werden:

Es ist wichtig den Durchführenden des Übungsprogramms einen klaren Weg vorzugeben.

Auf Grund dieser Erkenntnisse wäre es vielleicht ratsam, den Patientinnen unabhängig voneinander durchzuführende Übungsprogramme auszuhändigen, um die Wichtigkeit beider Belastungsformen deutlicher hervorzuheben. 


\section{Zusammenfassung}

Eine weiterhin steigende Anzahl von Tumorpatienten leidet unter der tumorbedingten Erschöpfung, der sogenannten tumorassoziierten Fatigue. Dies hat erhebliche Einschränkungen in der körperlichen und mentalen Belastbarkeit und der Lebensqualität zur Folge. Durch die zunehmende Anzahl von Fatiguepatienten nimmt auch die Anzahl von Studien über die Behandlungsmöglichkeiten der Fatigue zu. Im Rahmen der vorliegenden Interventionsstudie sollte die Einsetzbarkeit eines Heimübungsprogramms und die Auswirkung auf die tumorassoziierte Fatigue, die Lebensqualität und die Leistungsfähigkeit untersucht werden. Die Messungen erfolgten an vier Messzeitpunkten (T1-T4). Hierbei lagen die Messzeitpunkte T1 und T2 jeweils zu Beginn (T1) und am Ende (T2) der stationären Rehabilitation in der Sonnenbergklinik in Bad Sooden-Allendorf. Die Messzeitpunkte T3 und T4 lagen jeweils drei Monate (T3) und sechs Monate (T4) postrehabilitativ.

An den einzelnen Messzeitpunkten beantworteten die teilnehmenden Patientinnen unterschiedliche Fragebögen zur Fatigue und Lebensqualität (MFI und EORTC QLQ-C30), Angst und Depressionen (HADS) und einen Dokumentationsbogen mit sport- und physiotherapeutischen Fragestellungen sowie Angaben zur Person.

Zusätzlich füllten die Patientinnen der Trainingsgruppe einen Sportdokumentationsbogen aus, indem sie die Durchführung des Heimübungsprogramms in Intensität und Umfang festhielten. Auch die Durchführung aeroben Ausdauertrainings wurde von den Patientinnen der Trainingsgruppe dokumentiert.

Um eventuelle Veränderungen beider Gruppen während der stationären Rehabilitation zu entdecken, wurde zu Beginn und am Ende der Rehabilitation die isometrische Maximalkraft mittels einer Kraftmessdose ermittelt und die aerobe Ausdauerleistungsfähigkeit durch den 6 Min. Gehtest und dem PWC-Fahrradergometertest bestimmt.

Die Änderungen in beiden Gruppen in den Ergebnissen der Kraft- und Ausdauermessungen in der vorliegenden Studie sind primär auf koordinative Verbesserungen der Muskulatur zurückzuführen. Der PWC-Fahrradergometertest zeigte zum Messzeitpunkt T2 einen viel gleichmäßigeren Anstieg der Herzfrequenz als an T1, wo der Anstieg der Herzfrequenz ab 75 Watt unregelmäßig war. Der zeitliche Effekt von drei bis vier Wochen lässt eine Verbesserung der inter- und intramuskulären Koordination zu (Hollmann \& Hettinger, 2000).

Die Werte der Trainingsgruppe nach der Rehabilitation veränderten sich gegenüber den Werten zu Beginn der Rehabilitation signifikant in den Bereichen der allgemeinen Fatigue, 
gemessen durch den MFI (von 68,39 $\pm 19,93$ an T1 auf 45,58 $\pm 20,97$ an T4). Die Lebensqualität, gemessen durch den EORTC, veränderte sich von 50,42 $\pm 19,84$ an T1 auf $69,12 \pm 16,73$ an T4 (Global Health Status). Ängsten und Depressionen, gemessen durch den HADS änderten sich im Bereich der Angst an T1 von 8,8 $\pm 4,2$ auf $6,3 \pm 3,5$ an T4. Der Depressionsscore verringerte sich von 7,0 $\pm 4,1$ an T1 auf 5,9 $\pm 5,1$ an T4. Wenn es in der Kontrollgruppe zu Änderungen kam, waren diese nicht signifikant. Besonders in den Funktionsskalen des EORTC QLQ-C30 kam es in der Trainingsgruppe zu deutlichen Zeiteffekten in Verbindung mit sportlicher Aktivität. Alle Werte der Funktionsskalen des EORTC verbesserten sich signifikant von $\mathrm{T} 1$ bis $\mathrm{T} 4$. Doch auch die fünf Dimensionen des MFI zeigten die Unterschiede zwischen Kontrollgruppe und Trainingsgruppe deutlich auf. Alle Werte der einzelnen Dimensionen in der Trainingsgruppe an den Messzeitpunkten T3 und T4 waren signifikant niedriger gegenüber T1. Die allgemeine Fatigue des MFI verbessere sich sogar von T2 nach T3 und T4 signifikant, was für einen positiven Zeiteffekt von sportlicher Aktivität während und weiterhin nach der Rehabilitationsmaßnahme spricht. Besonders interessant ist die signifikante Reduktion der mentalen Fatigue der Trainingsgruppe von $63,93 \pm 24,1$ an T1 auf 37,69 $\pm 25,5$ an T4. Die signifikante Verbesserung der Motivation (reduzierte Motivation von 39,02 $\pm 20,7$ an T1 und 21,02 \pm 18 an T4) wirkte sich auch positiv auf das Aktivitätsniveau der Teilnehmerinnen der Trainingsgruppe aus. Der Score der reduzierten Aktivität der Trainingsgruppe lag zum Messzeitpunkt T1 bei 58,93 $\pm 24,1$ und zum

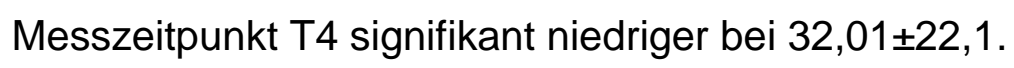

Interessant sind die positiven Veränderungen in der Trainingsgruppe im Bereich der körperlichen Selbsteinschätzung und subjektiven körperlichen Leistungsfähigkeit zu bewerten, gab es doch zu den Messzeitpunkten T3 und T4 mit 47,5\% und 52,9\% viele "gute" oder "sehr gute“ (5,9\% an T4) Einschätzungen der körperlichen Leistungsfähigkeit. Eine Verbesserung der körperlichen Leistungsfähigkeit bestätigte sich auch durch die Verbesserung beider Gruppen im 6 Min. Gehtest. Die Kontrollgruppe verbessere sich sogar signifikant von T1 $(510,7 \mathrm{~m})$ nach T2 $(540,6 \mathrm{~m})$. Doch auch die Trainingsgruppe verbesserte sich von 535,2m an T1 auf 553m an T2. Diese Ergebnisse lassen vermuten, dass auch die Rehabilitationsmaßnahmen sich positiv auf die körperliche Selbsteinschätzung und Leistungsfähigkeit auswirken.

Bei den Einschränkungen im täglichen Leben verbesserte sich die Trainingsgruppe gegenüber der Kontrollgruppe stark. 52,94\% (Trainingsgruppe) ohne Einschränkungen im täglichen Leben, standen 27,5\% (Kontrollgruppe) ohne Einschränkungen gegenüber, was 
deutlich macht, dass sportliche Aktivität auch die Leistungsfähigkeit im täglichen Leben verbessert.

Die Erkenntnisse vieler in der vorliegenden Arbeit zitierter Studien, konnten durch diese Studie bestärkt werden. Sportliche Aktivität wirkt sich während, aber auch besonders nach einer stationären, onkologischen Rehabilitation in vielen Bereichen positiv auf die Leistungsfähigkeit und die Lebensqualität der Patientinnen aus. 


\section{Danksagung}

Ich danke meinem Vater für die Finanzierung meines Studiums in Irland, ohne welches auch die Erstellung dieser Arbeit nicht möglich gewesen wäre.

Des Weiteren danke ich der Deutschen Krebshilfe für die finanzielle Förderung dieser Studie.

Prof. Dr. M.E. Heim und Prof. Dr. A. Niklas gilt mein besonderer Dank für die Betreuung und Beratung während der Studienzeit und während der Erstellung dieser Arbeit.

Prof. Dr. A. Krüger danke ich für die stets interessante Betreuung während des Doktorandenkolloquiums.

Der Sonnenbergklinik in Bad Sooden-Allendorf danke ich, dass ich die Studie dort durchführen und das Inventar der Klinik nutzen durfte.

Herrn Matthias Heim möchte ich danken, dass er sich die Zeit genommen hat, die erforderlichen Daten mit mir zu digitalisieren und auszuwerten.

Meiner Frau Juliane Wuttke gilt mein Dank für die Korrektur der Arbeit. 


\section{Literaturverzeichnis}

Aaronson, N., Ahmedzai, S., Bergman, B., Bullinger, M., Cull, A., Duez, N., et al. (3. März 1993). The european organization for Research and Treatment of cancer QLQ-C30: A quality of life instrument for use in international clinical trials in oncology. Journal of National Cancer Institute, 85 , S. 365-376.

Adamsen, L., Quist, M., Anderson, C., Moller, T., Herrstedt, J., Kronborg, D., et al. (8. Mai 2009). Effect of a multimodal high intensity exercise intervention in cancer patients undergoing chemotherapy: randomized controlled trial. BMJ, 339 , S. 1-11.

Alexander, S., Palmer, C., \& Stone, P. C. (4. Dezember 2009). Evaluation of screening instruments for depression and anxiety in breast cancer survivors. Breast Cancer Res Treat, 122(2), S. 573-578.

Bartsch, H. H., Weis, J., \& Moser, M. (2003). Cancer-Related Fatigue in Patients Attending Oncological Rehabilitation Programs: Prevalence, Patterns, Predictors. Onkologie, 26 , S. 51-57.

Bjelland, I., Dahl, A., Haug, T., \& Neckelmann, D. (9. Oktober 2002). The validity of the hospital anxiety and depression scale. An updated literature review. Journal of Psychosomatic Research, 52 , S. 69-77.

Blumenthal, J. A., Babyak, M. A., Moore, K. A., Craighead, E., Herman, S., Kathri, P., et al. (25. October 1999). Effects of exercise training on older patients with major depression. Archives of international medicine, 159 , S. 2349-2356.

Brenu, E., Staines, D., Baskurt, O., Ashton, K., Ramos, S., Rhys, C., et al. (11. Januar 2010). Immune and hemorheological changes in chronic fatigue syndrome. Journal of translational Medicine, 8 , S. 1-10.

Brezden, C., Phillips, K.-A., Abdolell, M., Bunston, T., \& Tannock, I. (July 2000). Cognitive Functioning In Breast Cancer Patients Receiving Adjuvant Chemotherapy. Journal of Clinical Oncology, 18(14), S. 2695-2701.

Carmack Taylor, C., Demore, C., Smith, M., Dunn, A., Basen-Engquist, K., Nielsen, I., et al. (31. Januar 2006). Active for life after cancer: A randomized trial examining a lifestyle physical activity program for prostate cancer patients. Psycho-Oncology, $15, \mathrm{~S}$. 847-862.

Cella, D. (1997). The functional assessment of cancer therapy-anemia (FACT-An) scale:a new tool for the assessment of outcomes in cancer anemia and fatigue. Seminars in hematology, 34(3), S. 9-13.

Courneya, K., Segal, R., Mackey, J., Gelman, K., Reid, R., Friedenreich, C., et al. (1. Oktober 2007). Effects of Aerobic and Resistance Exercise in Breast Cancer Patients Receiving Adjuvant Chemotherapy: A Multicenter Randomized Controlled Trial. Journal of Clinical Onkology, 25(28), S. 4396-4404. 
Cramp, F., \& Daniel, J. (2008). Exercise improves cancer-related fatigue. Cochrane Database of Systematic Review, 2 .

Cuijpers, P. (1997). Bibliotherapy in Unipolar Depression: A Meta-Analysis. J.Behav. Ther. \& Exp. Psychialt., 28(2), S. 139-147.

Daley, A. (15. April 2008). Exercise and Depression: A Review of Reviews. J Clin Psychol Med Settings, 15 , S. 140-147.

Deutsche Fatigue Gesellschaft. (2005). Fitness trotz Fatigue. Bonn: Eigendruck.

Deutsche Krebshilfe e.V. (2008). Fatigue. Chronische Müdigkeit bei Krebs. Bonn: Eigendruck.

Deutsche Rentenversicherung. (1. Dezember 2009). Rehabilitation. Abgerufen am 7. September 2010 von http://www.deutsche-rentenversicherung-bund.de

Deutscher Sportärztebund. (1996). Abgerufen am 10. März 2006 von Sport und Brustkrebs. Bewegung, Spiel und Sport bei Brustkrebs:

http://www.sportaerztebund.de/pub brustkrebs.htm

Dimeo, F., Fetscher, S., Lange, W., Mertelsmann, R., \& Keul, J. (1. November 1997). Effects of Aerobic Exercise on the Physical Performance and Incidence of TreatmentRelated Complications After High-Dose Chemotherapy. Blood, 90(9) , S. 3390-3394.

Dolbeault, S., A., B., V., M., Gauvain-Piquard, A., Mandereau, L., \& B., A. (Juni 2008). Screening for psychological distress in two French cancer centers: feasibility and performance of the adapted distress thermometer. Palliat Support Care, 6(2) , S. 107-117.

Duclos, M., Gouarne, C., \& Bonnemaison, D. (2003). Acute and chronic effects of exercise on tissue sensitivity to glucocorticoids. Journal of Applied Physiology, 94 , S. 869875.

Elsner von der Malsburg, M.-L. (2006). Untersuchung zur Einsetzbarkeit und Effektivität eines strukturieten Übungsprogramms in der Rehabilitation bei Brustkrebspatientinnen mit Fatigue. Göttingen: Cuvillier Verlag Göttingen.

Enright, P., \& Sherrill, D. (20. Mai 1998). Reference equations for the six-minute walk in healthey adults. Am J Respir Crit Care Med, 158 , S. 1384-1387.

Esterling, B., Kiecolt-Glaser, J., Bodnar, J., \& Glaser, R. (13. July 1994). Chronic stress, social support, and persistent alterrations in the natural killer cell response to cytokines in older adults. Health Psychol, 13 , S. 291-298.

Foley, A., Hillier, S., \& Barnard, R. (9. März 2010). Effectivness of Once-weekly gymbases exercise programms for older adults post discharged from day rehabilitation: a randomized controlled trial. Br J Sports Med, 10 , S. 1-9.

Froböse, I., Nellessen-Marks, G., \& Wilke, C. (2010). Training in der Therapie. München: Elsevier $\mathrm{GmbH}$. 
Goedendorp, M., Gielissen, M., Verhagen, C., Peters, M., \& Bleijenberg, G. (2008). Severe fatigue and related factors in cancer patients before the initiation of treatment. British Journal of Cancer, 99 , S. 1408-1414.

Gosselink, R., Rouffaer, L., Vanhelden, P., Piot, W., Troosters, T., \& Christiaens, M.R. (2003). Recovery of upper limb function after axillary dissection. Journal of Surgical Oncology, 83 , S. 204-211.

Grosser, M., Starischka, S., \& Zimmermann, E. (2008). Das neue Konditionstraining. München: BLV Buchverlag GmbH\&Co. KG.

Guyatt, G., Sullivan, M., Thompson, P., Fallen, E., Pugsley, S., Taylor, W., et al. (15. April 1985). The 6-minute walk: a new measure of exercise capacity in patients with chronic heart failure. Can Med Assoc J, 132, S. 919-923.

Haass, M., Zugck, C., \& Kübler, W. (2000). Der 6-Minuten-Gehtest: Eine kostengünstige Alternative zur Spiroergometrie bei Patienten mit Herzinsuffizienz. Zeitschrift für Kardiologie , 89 (2), S. 72-80.

Halle, Schmidt-Trucksäss, Hambrecht, \& Berg. (2008). Sporttherapie in der Medizin. Evidenzbasierte Prävention und Therapie. Stuttgart: Schattauer GmbH.

Headly, J., Ownby, K., \& John, L. (17. September 2004). The effect of seated exercise on fatigue and quality of life in women with advanced breast cancer. Oncol Nurs Forum, S. 977-983.

Heim, M., Elsner von der Malsburg, M.-L., \& Niklas, A. (2007). Randomized controlled trial of a structured training program in breast cancer patients with tumor-related chronic fatigue. Onkologie, 30 , S. 429-434.

Heim, M., Kunert, S., \& Özkan, I. (2001). Effects of inpatient rehabilitation health-related quality of life in breast cancer patients. Onkologie, 24 , S. 268-272.

Höffken, K. (2003). Den Brustkrebs besiegen.Rechtzeitig erkennen, richtig behandeln, heilen und leben. München: Ullstein Heyne List.

Hofmann, \& Kalj. (2004). Handbuch der betriebsärztlichen Praxis. Landsberg: Ecomed Medizin.

Hollmann, W. (1995). Lexikon der Sportmedizin. Heidelberg: Barth Verlag.

Hollmann, W., \& Hettinger, T. (2000). Sportmedizin. Grundlagen für Arbeit, Training und Präventivmedizin. Stuttgart: Schattauer.

Hollmann, W., \& Strüder, H. (2009). Sportmedizin. Grundlagen für körperliche Aktivität, Training und Präventivmedizin. Stuttgart: Schattauer. 
Hollmann, W., Strüder, H. K., Predel, H., \& Tagarakis, C. V. (2006). Spiroergometrie Kardiopulmonale Leistungsdiagnostik des Gesunden und Kranken . Stuttgart: Schattauer $\mathrm{GmbH}$.

Holmes, M., Chen, W., Feskanich, D., Kroenke, C., \& Colditz, G. (25. Mai 2005). Physical activity and survival after breast cancer diagnosis. The Journal of the American Medical Association, 293 , S. 2479-2786.

Holzner, B., Kemmler, G., Greil, R., Kopp, M., Zeimet, A., Raderer, M., et al. (20. 11 2002). The impact of hemoglobin levels on fatigue and quality of life in cancer patients. Annals of Oncology, 13 , S. 965-973.

Hsu, T.-G., Kuang, M., Kong, c., Fung, J., Cheng, H., \& Tsai, K. (Februar 2010). Lycocyte Mitochondria alterations after aerobic performance. Official Journal of the Americam College of sports medicine, 34(3) , S. 438-442.

Hüter-Becker, A., \& Dölken, M. (2005). Biomechanik, Bewegungslehre, Leistungsphysiologie, Trainingslehre. Stuttgart/ New York: Georg Thieme Verlag.

Hwang, J. H., Chang, H. J., Young, H. S., hah, P. W., Won, P., Jae, H. S., et al. (2008). Effects of supervised exercise therapy in patients recieving radiotherapy for beast cancer. Yonsei Med J, 49(3) , S. 443-450.

Israel, S. (1982). Sport und Herzschlagfrequenz. Leipzig: Barth.

Jean-Pierre, P., Figueroa-Moseley, C., Kohli, S., Fiscella, K., Palesh, O., \& Morrow, G. (2007). Assessment of Cancer-Related Fatigue: Implications for Clinical Diagnosis and Treatment. The Oncologist, 12, S. 11-21.

Kemmler, G., Holzner, B., Kopp, M., Dünser, M., Margreiter, R., Greil, R., et al. (27. Mai 1999). Comparison of Two Quality-of-Life Instruments for Cancer Patients: The Functional Assessment of Cancer Therapy-General and the European Organization for Research and Treatment of Cancer Quality of Life Qustionnaire C30. Journal of Clinical Oncology, 17 , S. 2932-2940.

Krebshilfe, D. (2003). Fatigue. Chronische Müdigkeit bei Krebs. Bonn: Eigendruck.

Krostjens, I., Mesters, I., van der Peet, E., Gijsen, B., \& van den Borne, B. (Dezember 2006). Quality of life of cancer survivors after physical and psychosocial rehabilitation. Eur J Cancer Prev, 15(6) , S. 541-547.

Kuehn, T., Klauss, W., Darsow, M., Regele, S., Flock, F., Maiterth, C., et al. (2000). Long-term morbidity following axillary dissection in breast cancer patients - clinical assessment, significance for life quality and the impact of demographic, oncologic and therapeutic factors. Breast Cancer Research and Treatment, 64 , S. 275-286.

Kuhnt, S., Ernst, J., Singer, S., Rüffer, J. U., Kortmann, R. D., Stolzenburg, J. U., et al. (2009). Fatigue in cancer survivors - Prevalence and Correlates. Onkologie, 32 , S. 312-317. 
Künstlinger, U. (2002). Bedeutung der körperlichen Aktivität für kardiale und zerebrale Funktionen. Am Beispiel der Primärprävention, Kardiologie, Onkologie und Psychatrie. Deutsche Zeitschrift für Sportmedizin, 53(1) , S. 27-28.

Lötzerich, H., \& Uhlenbrock, G. (1991). Sport und Immunologie. In M. Weiss, \& H. Rieder, Sportmedizinische Forschung. Berlin: Springer.

Mc Tiernan, A. (2004). Physical activity after cancer: Physiologic outcomes. Cancer Invest, 22(1), S. 68-81.

McNeely, M., Campbell, C., Ospina, M., Rowe, B., Dabbs, K., Klassen, T., et al. (2010). Exercise interventions for upper-limb dysfunction due to breast cancer treatment. The Cochrane Collaboration, 6 , 1-75.

Mellerowicz, H. (1975). Zusammenfassung zur proaktischen Ergometrie. In H. Mellerowicz, Ergometrie (S. 337-348). Berlin: Urban und Schwarzenberg.

Mock, V., \& Watson, T. (August 2004). Exercise as an intervention for cancer-related Fatigue. Physical Therapy, 84 , S. 736-743.

Montazeri, A. (29. 8 2008). Health-related quality of life in breast cancer patients: A bibliographic review of the literature from 1974 to 2007. Journal of Experimental \& Clinical Cancer Research, 27 , S. 32-63.

Montazeri, A., Vahdaninia, M., Harirchi, I., Ebrahimi, M., Kahleghi, F., \& Jarvandi, S. (11. November 2008). Quality of life in patients with breast cancer before and after diagnosis: an eighteen months follow-up study. BMC Cancer, 8 , S. 330-336.

Mooren, F., Lechtermann, A., \& Völker, K. (März 2010). Exercise-induced apoptosis of Lymphcytes depends on training status. Medicine \& Science in Sports \& Exercise, 36(9), S. $1476-1483$.

Mustian, K., Griggs, J., Morrow, G., McTiernan, A., Roscoe, J., Bole, C., et al. (16. Februar 2006). Exercise and side effects among 749 patients during and after treatment for cancer: a University of Rochester Cancer Center Community Clinical Oncology Program Study. Support Care Cancer, 14 , S. 732-741.

Nabkarson, C., Miyai, N., Sootmongkol, A., Junprasert, S., Yamamoto, H., Arita, M., et al. (26. August 2005). Effects of physical exercise on depression, neuroendocrine stress hormones and physiological fitness in adolescent females with depressive symptoms. European Journal of Public Health, 16(2), S. 179-184.

Navalta, J., Mohamed, Refaat, El-Baz, A., McFarlin, B., \& Lyons, S. (3. Mai 2010). Exercise-induced immune cell apoptosis: image-based model for morphological assessment. Eur J Appl Physiol, 10 , S. 325-331.

NICE. (2007). Management of depression in primary and secondary care. Abgerufen am 13. Juli 2010 von National Institiute for Clinical Excellence:

http://www.nice.org.uk/nicemedia/pdf/CG023fullguideline.pdf 2007. 
Oschütz, H. (1998). Zirkadianrhythmische Aspekte muskulärer Beanspruchung und wiederherstellungsfördernder Maßnahmen. In L. Zichner, M. Engelhardt, \& J. Freiwald, Die Muskulatur. Sensibles, integratives und meßbares Organ (S. 173-180). Nürnberg: Novartis Pharma Verlag.

Port, E. R., Patil, S., Stempel, M., Morrow, M., \& Cody, H. (15. April 2010). Number of Lymph Nodes Removed in Sentinel Lymph Node-Negative Breast Cancer Patients Is Significantly Related to Patient Age and Tumor Size. Cancer. American Cancer Society, 116, S. 1987-1991.

Radlinger, L. (1998). Rehabilitative Trainingslehre. Stuttgart/New York: Gearg Thieme Verlag.

Raschka, C., \& Vogel, M.-L. (2010). Herzsport. Wiebelsheim: Limperl Verlag.

Reuter, K., Classen, C. C., Roscoe, J. A., Morrow, G. R., Kirshner, J. J., Rosenbluth, R., et al. (19. Dezember 2006). Association of coping style, pain, age and depression with fatigue in women with primary breast cancer. Psycho-Oncology, 15 , S. 772-779.

Riede, U.-N., \& Schäfer, H. E. (2001). Allgemeine und spezielle Pathologie. Stuttgart, New York: Georg Thieme Verlag.

Rühl, J., \& Schube, V. (2003). Funktionelles Fitnesskrafttraining. Aachen: Meyer\&Meyer Verlag.

Schmidt, R., \& Thews, G. (1987). Physiologie des Menschen. Berlin: Springer Verlag.

Schmitz, K., Courneya, K., Matthews, C., Demark-Wahnefried, W., Galvao, D., Pinto, M., et al. (July 2010). American College of Sports Medicine roundtable on exercise guidelines for cancer survivors. Med Sci Sports Exerc, 42(7) , S. 1409-1426.

Schulz, K., \& Heesen, C. (2005). Auswirkung körperlicher Aktivität bei chronisch Kranken. Beispiele aus der Onkologie und Neurologie. Bundesgesundheitsbl-GesundheitforschGesundheitsschutz, S. 906-913.

Schünemann, H., Ascher, G., \& Jänicke, F. (1993). Brustkrebs und Sport. Deutsche Zeitschrift für Sportmedizin, 44(19) , S. 491-503.

Schwarz, R., Krauss, O., \& Hinz, A. (2003). Fatigue in the general population. Onkologie, 26 , S. $140-144$.

Singer, S., Kuhnt, S., Götze, H., Hauss, J., Hinz, A., Liebmann, A., et al. (24. Februar 2009). Hospital anxiety and depression scale cutoff scores for cancer patients in acute care. British Journal of Cancer, 100 , S. 908-912.

Smets, E., Garrsen, B., Cull, A., \& de Haes, J. (1996). Application of the multidimensional fatigue inventory (MFI-20) in cancer patientsrecieving radiotherapy. British Journal of Cancer , S. 241-245. 
Smets, E., Garssen, B., A, C., \& de Haes, J. (1996). Application of the multidimensional fatigue inventory (MFI-20) in cancer patients receiving radiotherapy. British Journal of Cancer, 73, S. 241-245.

Spence, R., Heesch, K., \& Brown, W. (April 2010). Exercise and cancer rehabilitation: a systematic review. Cancer Treat Rev, 36 , S. 185-194.

Springer, B. A., McGarvey, C., Pfalzer, L. A., Stout, N. L., Gerber, L. H., Soballe, P. W., et al. (2010). Pre-operative assessment enables early diagnosis and recovery of shoulder function in patients with breast cancer. Breast Cancer Res Treat, 120 , S. 135147.

Sprod, L., Hsieh, C., Hayward, R., \& Schneider, C. (5. Mai 2010). Three versus six months of exercise training in breast cancer survivors. Breast Cancer Res Treat, 121 , S. 413-419.

Steffen, T., Hacker, T., \& Mollinger, L. (2002). Age-and Gender related test performance in community-dwelling elderly people: six-minute walk test, berg balance scale, timed uo and go test and gait speeds. Physical Therapy, 82 , 128-137.

Stein, \& Greitmann. (2005). Rehabilitation in Orthopädie und Unfallchirurgie. Heidelberg: Springer Medizin Verlag.

Stemper, T. (1988). PWC, Cardio-Fitness-Test. In T. Stemper, Gesundheit-FitnessFreizeitsport (S. 1-13). Hamburg: SSV-Verlag.

Straus, D., Testa, M., Sarokhan, B., Czuczmann, M., Tulpule, A., Turner, R., et al. (15. Oktober 2006). Quality-of-life and health benefits of early treatment of mild anemia. CANCER, 107(8) , S. 1909-1917.

Strömbeck, B., Theander, E., \& Jacobssen, L. (17. Februar 2007). Effects of exercise on aerobic capacityand fatigue in women with primary Sjögren's syndrome.

Rheumatology, 46 , S. 868-871.

Terson de Palleville, S., Topp, R. V., \& Swank, A. (2007). Effects of aerobic training prior to and during Chemotherapy in breast cancer patients. A case study. Journal of Strength and Conditioning Research, 21(2) , S. 635-637.

Timmons, B., \& Cieslak, T. (2008). Human natural killer cell subsetsand acute exercise: a brief review. Exerc Immunol Rev, 14, S. 8-23.

Valenti, M., Porzio, G., Aielli, F., Verna, L., Cannita, K., \& Renato. (Januar 2008). Physical Exercise and Quality of Life in Breast Cancer Survivors. International Journal of Medical Sciences, 5(1) , S. 24-28.

Van Weert, E., May, A., Krostjens, I., Post, W., van der Schans, C., van den Borne, B., et al. (22. Julie 2010). Cancer-related fatigue and rehabilitation: A randomized controlled multicenter trial comparing physical training combined with cognitive-behavioral therapy with physical training alone and with no intervention. Phys Ther, 10, S. 1413-1425. 
Visser, M., \& Smets, E. (1998). Fatigue, depression and quality of life in cancer patients: How are they related. Suppport Care Cancer, 6(2) , S. 101-108.

Wiggins, M., \& Simonavice, E. (2008). Qualitiy of Life Benefits: A 12 Month Exercise and Cancer Recovery Case Study. KAHPERD Journal, 7, S. 16-19.

Wingate, L. (June 1985). Efficacy of Physical Therapy for Patients Who Have Undergone Mastectomies. Physical Therapy, 65, S. 896-900.

Wirz, M. (2006). Timed Walking Test. Physiopraxis, 93 , 36-37.

Witek-Janusek, L., Gabram, S., \& Mathews, H. (Januar 2007). Psychologic stress, reduced NK cell activity, and cytokine dyregulation in women experiencing diagnostic breast biopsy. Psychoneuroendocrinology, 32(1) , S. 22-35. 


\section{Anlagen}

Anlage 1: Informationsschreiben

\section{Patienteninformationen}

Sehr geehrte Patientinnen,

Sie befinden sich zurzeit in einer Rehabilitationsbehandlung bzw. Anschlussheilbehandlung in der Sonnenberg-Klinik in Bad Soden-Allendorf. Sie erhalten hier alle notwendigen und geeigneten Untersuchungen und Behandlungen, die wesentlichen Besserung oder Wiederherstellung Ihrer Gesundheit und Leistungsfähigkeit beitragen. Wir sind ständig bemüht, unsere Behandlungsangebote den neusten Erkenntnissen anzupassen und auch einen Beitrag hierzu zu leisten. Deshalb möchten wir mit dem Forschungsvorhaben „, Untersuchung zur Einsetzbarkeit und Effektivität eines strukturierten Übungsprogramms in der Rehabilitation bei Brustkrebspatienten mit Fatigue“, das in Kooperation mit dem Institut für Sportwissenschaften der Universität Göttingen in unserer Klinik durchgeführt wird, prüfen, ob eine Verbesserung der Behandlung durch ein strukturiertes Bewegungsprogramm möglich ist.

Viele Patienten fühlen sich nach der Erkrankung und deren Therapie erschöpft und ihre Lebensqualität ist stark beeinträchtigt. Mit Hilfe eines speziellen Übungsprogramms, welches sowohl aus Kraft- und Ausdauerübungen, als auch Entspannungsübungen besteht, möchten wir untersuchen, inwieweit sportliche Aktivität Ihre „Fatigue“ (Müdigkeit) lindern und Ihre Leistungsfähigkeit steigern kann.

Die Untersuchung soll dazu beitragen, Rehabilitationsmaßnahmen bei Frauen mit Brustkrebs noch effektiver einsetzen zu können, um die gesundheitsbezogene Lebensqualität zu verbessern.

Wir würden uns daher freuen, wenn Sie sich an dem Projekt beteiligen würden, das Übungsprogramm regelmäßig ausführen und die Fragen und Ihr Trainingsprotokoll sorgfältig und vollständig ausfüllen. Es ist geplant, an 4 Messzeitpunkten (T1-T4), d.h. am Anfang (T1) und am Ende (T2) der stationären Rehabilitation sowie nach 3 (T3) und 6 (T4) Monaten nach Entlassung aus der Klinik jeweils einen Fragebogen auszufüllen, der anschließend anonym ausgewertet wird. An den ersten beiden Messzeitpunkten werden zusätzlich zwei einfache Leistungstests durchgeführt, die zum einen die Veränderung der Ausdauer und Kraft messen und zum anderen die Leistungsfähigkeit es Herz-Kreislauf-Systems bestimmen. Zum dritten und vierten Messzeitpunkt wird Ihnen jeweils der Fragebogen zusammen mit einem frankierten Rückumschlag zugeschickt, so dass Sie den Bogen ausfüllen und mit Ihrem Fitnessbogen an uns zurückschicken können. Zu diesem Zeitpunkt wird kein Leistungstest mehr durchgeführt. Neben den Daten, die durch Fragebögen und Leistungstests erhoben werden, werden zusätzlich einige biographische Daten (u.a. Alter, Familienstand) sowie Daten zur Brusterkrankung und zur Behandlung durch den Doktoranden aus der Krankenblattakte 
entnommen. Der Doktorand arbeitet an dem Forschungsvorhaben mit und ist wie auch das Klinikpersonal zur Verschwiegenheit verpflichtet.

Die Untersuchung bringt weder gesundheitliche Risiken noch entstehende Kosten für Sie mit sich. Alle im Rahmen der klinischen Prüfung anfallenden Daten unterliegen dem Datenschutz.

Die Fragebögen werden anonym ausgewertet. Die Anonymisierung erfolgt in der Klinik durch die Vergabe einer Nummer, so dass die Zuordnung der Fragebögen zu Namen mit Anschriften nur über eine Liste erfolgen kann, die in der Klinik verbleibt. Die Liste der Nummern und den dazugehörigen Namen und Anschriften wird nur bis zu dem Zeitpunkt aufbewahrt, bis wir Sie nicht mehr kontaktieren müssen. Sie wird nach dem 4. Messzeitpunkt, an dem wir Ihnen einen Fragebogen nach Hause schicken vernichtet. Die Liste mit Namen und Anschriften ist nur dem zuständigen Klinikpersonal zugänglich. Nach Vernichtung der Liste lässt sich auch in unserer Klinik keine Verbindung von Forschungsnummern zu den Teilnehmern mehr herstellen. Bei einer Veröffentlichung durch das Forschungsinstitut lässt sich kein Bezug zu Ihrer Person mehr herstellen.

Es werden in Zusammenhang mit dieser Studie keine personenbezogenen Daten (Namen, Geburtsdatum, Adresse oder sonstige Angaben, die Rückschlüsse auf die Person zulassen) an Dritte oder andere Institutionen weitergegeben. Die Projektleiter gewährleisten die Vertraulichkeit aller Informationen.

Die Teilnahme an der Studie ist für Sie freiwillig und Sie können jederzeit, auch bei schon gegebener Einwilligung, Ihre Zustimmung zur Teilnahme ohne Nennung von Gründen zurückziehen. Auch wenn Sie die Teilnahme ablehnen, erwachsen Ihnen hieraus keine Nachteile. Wenn Sie zu einem späteren Zeitpunkt aus dem Forschungsvorhaben ausscheiden möchten, teilen Sie dies bitte schriftlich unserer Klinik mit. Wir werden dann Ihren Namen in der oben beschriebenen Liste unkenntlich machen und Sie nicht mehr anschreiben. Sie können sich darauf verlassen, dass Ihre Daten nur verwendet werden, wenn Sie die Einverständniserklärung unterschrieben haben. Wenn Sie das Vorangegangene gelesen haben, Ihnen der Inhalt klar ist und Sie an dieser Studie teilnehmen möchten, bitten wir Sie, die beiliegende Einverständniserklärung zu unterschreiben. Die Untersuchung erfolgt im eigenen Interesse der Klinik und wurde nicht von der BfA veranlasst.

Mit freundlichen Grüßen,

Prof. Dr. med. M.E. Heim 
Anlage 2: Einwilligungserklärung zur Studie

Einwilligungserklärung zur Studie:

Untersuchung zur Einsetzbarkeit und Effektivität eines strukturierten Übungsprogramms in der Rehabilitation bei Brustkrebspatienten mit Fatigue

Name der Patientin

Zimmernr.

Ich wurde von meinem Arzt über Wesen, Bedeutung und Tragweite des oben genannten Forschungsprojektes aufgeklärt. Ich habe die Patienteninformation gelesen und verstanden. Ich hatte die Möglichkeit, Fragen zu stellen, habe die Antworten verstanden und akzeptiere sie. Mein Arzt hat mich über die mit der Teilnahme an der Studie verbundenen Risiken und den möglichen Nutzen informiert.

Ich hatte ausreichend Zeit, mich zur Teilnahme an dieser Untersuchung zu entscheiden und weiß, dass die Teilnahme an dieser Untersuchung freiwillig ist. Ich weiß, dass ich jederzeit und ohne Angabe von Gründen diese Zustimmung wiederrufen kann, ohne dass sich dieser Entschluss auf die spätere Behandlung durch meinen Arzt auswirken wird.

Insbesondere bi ich damit einverstanden, dass Klinikmitarbeiter Diagnosen, Medikation und Behandlungsergebnisse aus der Krankenblattakte entnehmen und unter einer Forschungsnummer an das wissenschaftliche Forschungsinstitut übermitteln. Ich entbinde die Klinikmitarbeiter insoweit von ihrer Schweigepflicht.

Mir wurde versichert, dass keine personenbezogenen Angaben (Name, Geburtsdatum, Adresse oder sonstige Angaben, die Rückschlüsse auf meine Person zulassen) an Dritte weitergegeben werden und dass im Zusammenhang mit dieser Untersuchung erhobene Daten gelöscht werden, sobald sie für die weitere wissenschaftliche Auswertungen nicht mehr erforderlich sind.

Hiermit erkläre ich mich bereit, an der geplanten Untersuchung unter den im Aufklärungsschreiben genannten Vorrausetzungen teilzunehmen.

Ort und Datum

Unterschrift des Patienten 
Anlage 3: Testprotokoll 6 Min. Gehtest

Testprotokoll 6 min. GT

\begin{tabular}{|c|c|c|c|}
\hline Name: & & Testdatum: & Abreise: \\
\hline Geb.: & Größe: & Gew.: & \\
\hline Zeit: & Gehstrecke: & Puls: & $\begin{array}{l}\text { Blut- } \\
\text { druck: }\end{array}$ \\
\hline \multicolumn{4}{|l|}{ Ausgang } \\
\hline \multicolumn{4}{|l|}{2 Min. } \\
\hline \multicolumn{4}{|l|}{4 Min. } \\
\hline \multicolumn{4}{|l|}{6 Min. } \\
\hline \multicolumn{4}{|l|}{$\begin{array}{c}\text { Nach } 2 \\
\text { Min. }\end{array}$} \\
\hline \multicolumn{4}{|l|}{$\begin{array}{c}\text { Nach } 4 \\
\text { Min. }\end{array}$} \\
\hline & & & \\
\hline & & & \\
\hline & & & \\
\hline & & & \\
\hline & & & \\
\hline
\end{tabular}


Anlage 4: Testprotokoll PWC-Fahrradergometertest

Testprotokoll PWC 150

\begin{tabular}{|c|c|c|c|}
\hline Name: & & Testdatum: & Abreise: \\
\hline Geb.: & Größe: & Gew.: & \\
\hline Zeit: & Watt: & Puls: & $\begin{array}{l}\text { Blut- } \\
\text { druck: }\end{array}$ \\
\hline \multicolumn{4}{|l|}{ Ausgang } \\
\hline 3 Min. & 25 & & \\
\hline 6 Min. & 50 & & \\
\hline 9 Min. & 75 & & \\
\hline 12 Min. & 100 & & \\
\hline $15 \mathrm{Min}$. & 125 & & \\
\hline $18 \mathrm{Min}$. & 150 & & \\
\hline $21 \mathrm{Min}$. & 175 & & \\
\hline 24 Min. & 200 & & \\
\hline nach 2 Min. & 50 & & \\
\hline nach2 Min. & 25 & & \\
\hline
\end{tabular}


Name des Patienten:

Zimmernummer:

Wie erschöpft fühlen Sie sich?

Bitte machen Sie ein Kreuz auf der Stelle der Skala, die nach Ihrer eigenen Einschätzung Ihren aktuellen Erschöpfungszustand beschreibt!
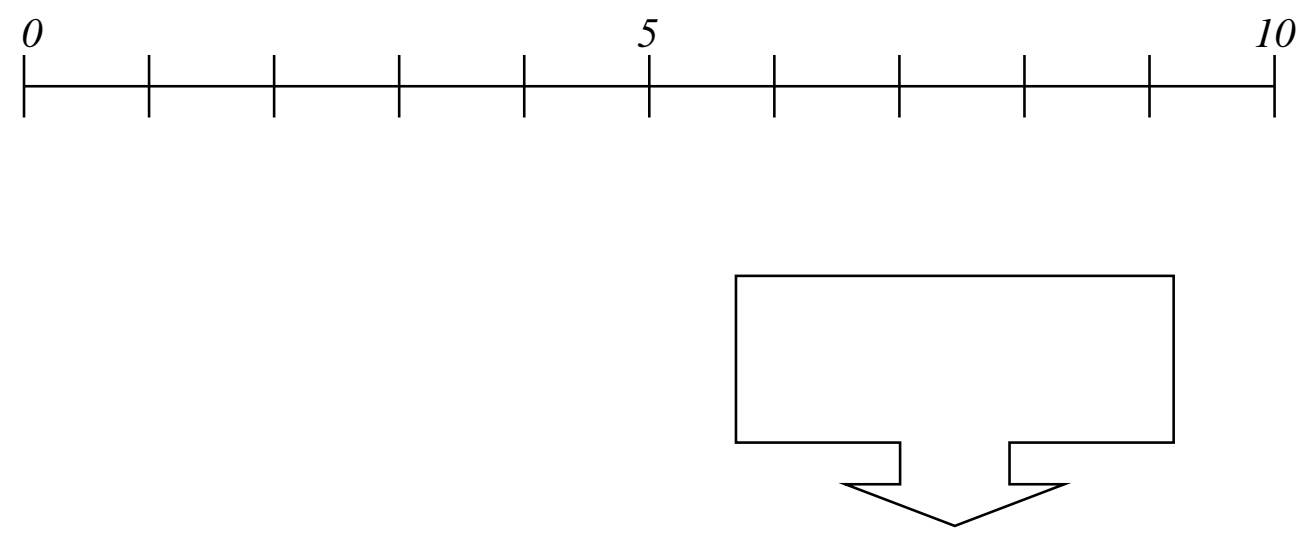

Falls das Kreuz auf der Skala im Bereich 4-10 liegt, möchten wir Sie gerne am Donnerstag um 16.00 Uhr zu einer Informationsveranstaltung in die Bibliothek im Erdgeschoss einladen.

Bitte geben Sie den Zettel in jedem Fall ausgefüllt im Stationszimmer ab, auch wenn Sie nicht an Erschöpfung leiden bzw. nicht zur Informationsveranstaltung kommen möchten.

Vielen Dank! 
Anlage 6: Dokumentationsbogen:

\section{Dokumentationsbogen}

Sporttherapie zur Behandlung des Fatigue-Syndroms bei Patientinnen mit Zustand nach MammaCa-Operation

Prof. Dr. med. M. Heim

Chefarzt der Sonnenbergklinik Bad Soden-Allendorf

Erhebungszeitpunkt: Beginn der stationären Rehabilitation (t1)

Teilnehmer-Nr.:

Dieser Fragebogen wurde an folgendem Datum ausgefüllt:

$\overline{\text { Tag }} \quad \overline{\text { Monat }} \quad \overline{\mathrm{Jahr}}$ 


\section{Soziale Anamnese und allgemeine Fragen zur Person:}

Alter:

Aktuelle berufliche Situation:

- Hausfrau

○ Ganztagsbeschäftigung

○ Teilzeitbeschäftigung

- Selbstständig

○ Rentnerin, Ruhestand

Sonstiges: (bitte angeben)

Größe:

Gewicht:

Raucher

Nichtraucher

Wenn Raucher:

Seit wie viel Jahren?

Seit welchem Lebensjahr?

Wie viele Zigaretten pro Tag?

\section{Fragen zur Brusterkrankung und Behandlung}

Datum der Erkrankung (Diagnose):

Monat:

Jahr:

Datum der Erstbehandlung:

Monat: Jahr:

Welche Art der Erstbehandlung wurde durchgeführt? (bitte ankreuzen)

$\begin{array}{ll}\text { Operation } & \mathrm{O} \\ \text { Wenn ja: } & \text { M } \\ & \text { Brie } \\ & \\ & \mathrm{O} \\ & \mathrm{O} \\ & \mathrm{O}\end{array}$

Sonstige Therapieformen? 
Laufen zurzeit noch spezifische Behandlungen?Ja $\quad \mathrm{O} \quad$ Nein $\quad \mathrm{O}$

Wenn ja, welche?

Chemotherapie $\quad \mathrm{O}$

Hormontherapie $\quad \mathrm{O}$

Schmerztherapie O

Antikörpertherapie $\mathrm{O}$

Andere

Ist nach der Entfernung der Brust ein weiterer Tumor (Lokalrezidiv) aufgetreten und behandelt worden? Ja O Nein O

Sind Tochtergeschwülste (Metastasen) im Organismus aufgetreten bzw. behandelt worden?

$\mathrm{Ja} \quad \mathrm{O}$ Nein $\mathrm{O}$

Stationäre Rehabilitation aufgrund der Brustbehandlung bereits zu einem früheren Zeitpunkt?
$\mathrm{Ja}$
$\mathrm{O}$
Nein $\mathrm{O}$

Wenn ja, wann?

\section{Physiotherapeutischer Fragebogen}

Sind die Operationsnarben schmerzhaft?

Sind Schwellungen im OP-Bereich vorhanden?

Sind schmerzhafte Muskelverspannungen im Schulter-Nacken-Bereich vorhanden?

$$
\mathrm{Ja} \quad \mathrm{O}
$$

Ist die Atmung erschwert oder schmerzhaft?

Oberarm

Unterarm

Hand
$\mathrm{Ja} \quad \mathrm{O}$

$\mathrm{Ja} \quad \mathrm{O}$

Nein $\mathrm{O}$

$\begin{array}{llll}\mathrm{Ja} & \mathrm{O} & \text { Nein } & \mathrm{O} \\ \mathrm{Ja} & \mathrm{O} & \text { Nein } & \mathrm{O}\end{array}$

Nein $\mathrm{O}$

Nein O

Nein $\mathrm{O}$

Nein $\mathrm{O}$

vorhanden?

Ist ein gefühlter Kraftverlust im Arm oder Schulter vorhanden?

$$
\begin{array}{lll}
\mathrm{Ja} & \mathrm{O} & \text { Nein } \mathrm{O}
\end{array}
$$

Sind Bewegungseinschränkungen und Einschränkungen im täglichen Leben vorhanden?

Ja O Nein O 
Wenn ja, wo? (z.B. Schulter, Arm; Dinge heben, halten etc.)

\section{Fragen zur sportlichen Aktivität}

Betrieben Sie vor Ihrer Erkrankung Sport?

Nein, gar nicht $\quad \mathrm{O}$

Unregelmäßig $\mathrm{O}$

Ja, regelmäßig $\quad \mathrm{O}$

Wenn ja: Welche Sportarten?

Aus welcher Motivation treiben Sie Sport?

Gesundheit

$\mathrm{O}$

Spaß

$\mathrm{O}$

Ausgleich

$\mathrm{O}$

Geselligkeit

$\mathrm{O}$

Andere

Haben Sie während Ihrer Therapie Sport betrieben?

Nein, gar nicht

$\mathrm{O}$

Unregelmäßig

$\mathrm{O}$

Ja, regelmäßig

$\mathrm{O}$

Wenn ja: Welche Sportarten?

Std. pro Woche

seit wann?

Wie würden Sie Ihre aktuelle körperliche Leistungsfähigkeit einschätzen?

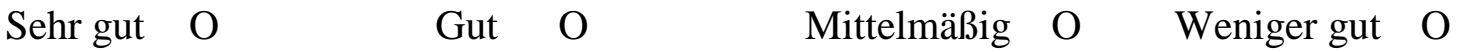

Schlecht O 
Anlage 7: Ergometrie

\section{Was ist Ergometrie?}

Ergometrie bedeutet in wörtlicher Übersetzung Leistungsmessung (ergon $=$ griech. das Werk, die Arbeit, die Leistung). Bei der Ergometrie wird im Gegensatz zu anderen Funktionsprüfungen die Leistung des Körpers physikalisch exakt in den international gebräuchlichen Leistungsgrößen $\mathrm{mkp} / \mathrm{sec}$ bzw. Watt gemessen $(1 \mathrm{mkp} / \mathrm{sec}=9,81$ Watt $\approx 10$ Watt.

Die ergometrisch gemessene Leistung ist genau vergleichbar und reproduzierbar. Doch müssen hierfür bestimmte, jetzt international definierte Leistungsumsatzbedingungen (s. III.2) eingehalten werden (Temperatur im Untersuchungsraum, Ernährung am Untersuchungstage u. a. betr.).

Bei den für die Ergometrie erforderlichen Leistungsmeßgeräten werden mechanisch und elektromagnetisch gebremste Ergometer unterschieden. Die ergometrische Leistung ist bei beiden Formen das Produkt aus Bremskraft in $1 \mathrm{sec}$ (Leistung ist physikalisch $\frac{\mathrm{Kraft} \times \text { Weg }}{\text { Zeit }}$. Bei der Eichung der Ergometer wird die Leistung auf Meßskalen in mkp/sec bzw. in Watt angegeben. Oder sie ist aus Tabellen nach dem gemessenen Bremswiderstand, der Drehzahl und der Zeit ablesbar.

Die ergometrische Leistung kann in Form von Handkurbelarbeit im Stehen oder Fußkurbelarbeit im Sitzen oder Fußkurbelarbeit im Liegen produziert werden. Bei gleicher physikalischer Leistung von z. B. 100 Watt differiert bei diesen drei ergometrischen Leistungsformen die biologische Leistung etwas. Vergleichswerte wurden bestimmt (s. I., 4).

Bei gleicher physikalischer Leistung kann die biologische Leistung unterschiedlich sein in Abhängigkeit auch von der Drehzahl, der Kurbellänge, der Kurbelhöhe, der Untersuchungsmethodik u. a. Eine Standardisierung der Methodik war deshalb erforderlich. Sie wurde inzwischen von verschiedenen Ergometrie-Arbeitskreisen in Deutschland und auch international durchgeführt. (s. III.)

Quelle: (Mellerowicz, 1975). 
Die Herzschlagfrequenz während einer relativ gleichen Leistung von $1 \mathrm{Watt} / 1 \mathrm{~kg}$ Körpergewicht wird mit den Mittelwerten und der Standardabweichung verglichen (s. Abb. 189). Je höher die Herzschlagfrequenz ist, um so kleiner ist die kardio-korporale Leistungsbreite, je kleiner die Herzschlagfrequenz, um so größer ist die kardio-korporale Leistungsbreite. Diese einfache ergometrische Methode erlaubt bereits in 3, 4, 5 bis 6 Minuten in vielen Fällen eine brauchbare und hinreichend zuverlässige Beurteilung der kardio-korporalen Leistungsbreite.

Man mißt die Herzschlagfrequenz am einfachsten auskultatorisch oder palpatorisch mit einer Stoppuhr. Zwischen der 50. und 60. Sekunde jeder Minute wird die Zeitdauer von 10 Herzschlägen gestoppt und daraus die Minutenfrequenz errechnet bzw. aus einer Tabelle entnommen (Tab. 54).

Oder man zählt die Herzschläge bzw. Pulszahlen innerhalb 6 Sekunden und multipliziert sie mit 10, um die Minutenfrequenz zu erhalten.

Genauere Werte erhält man, wenn man $10 \mathrm{R}$-Zacken zwischen der 50 und 60. Sekunde jeder Minute elektrokardiographisch registriert und aus der Zeitdauer die Minutenfrequenz errechnet.

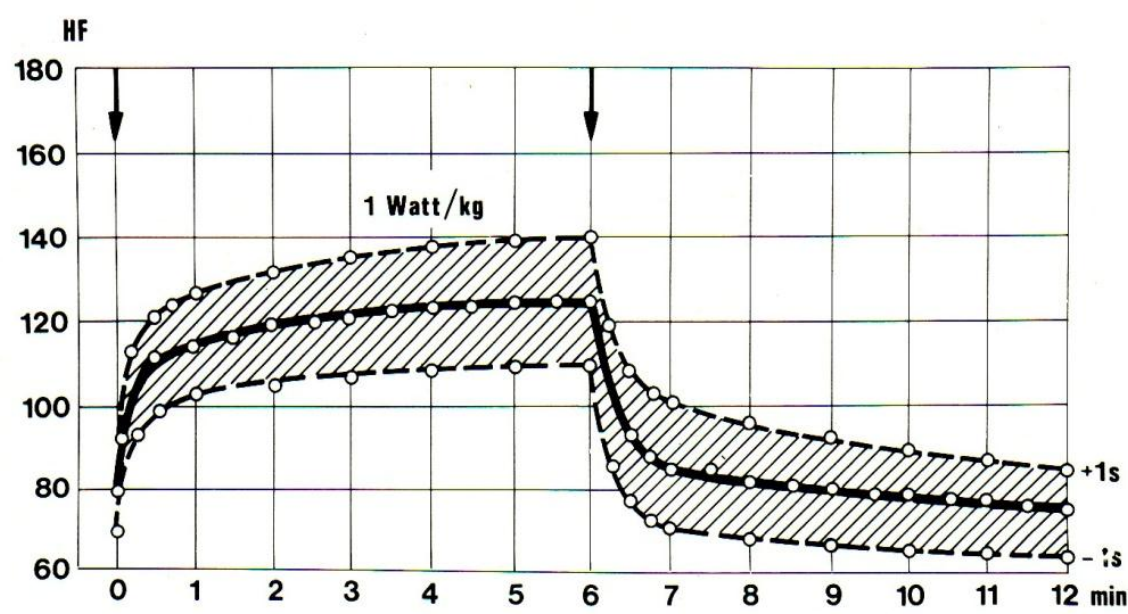

Quelle: (Mellerowicz, 1975). 


\begin{tabular}{|l|l|l|l|l|l|l|l|l|l|l|}
\hline \multicolumn{1}{|l|}{ Frauen [Alter in Jahren] } \\
\hline & $20-24$ & $25-29$ & $30-34$ & $35-39$ & $40-44$ & $45-49$ & $50-54$ & $55-59$ & $60-64$ \\
\hline Gewicht [kg] & Watt \\
\hline $58-61$ & 130 & 125 & 125 & 120 & 115 & 115 & 105 & 100 & 100 \\
\hline $62-65$ & 135 & 135 & 130 & 125 & 120 & 120 & 110 & 110 & 105 \\
\hline $66-69$ & 140 & 140 & 135 & 130 & 130 & 125 & 120 & 115 & 110 \\
\hline $70-73$ & 150 & 145 & 140 & 135 & 130 & 130 & 125 & 120 & 115 \\
\hline $74-77$ & 155 & 150 & 145 & 140 & 135 & 135 & 130 & 125 & 120 \\
\hline$\geq 78$ & 160 & 155 & 150 & 150 & 145 & 140 & 135 & 130 & 130 \\
\hline
\end{tabular}

Maximale Leistungsfähigkeit (Watt) in Abhängigkeit von Alter, Gewicht und Geschlecht (hier Frauen) nach Reiterer. Quelle: (Hofmann \& Kalj, 2004).

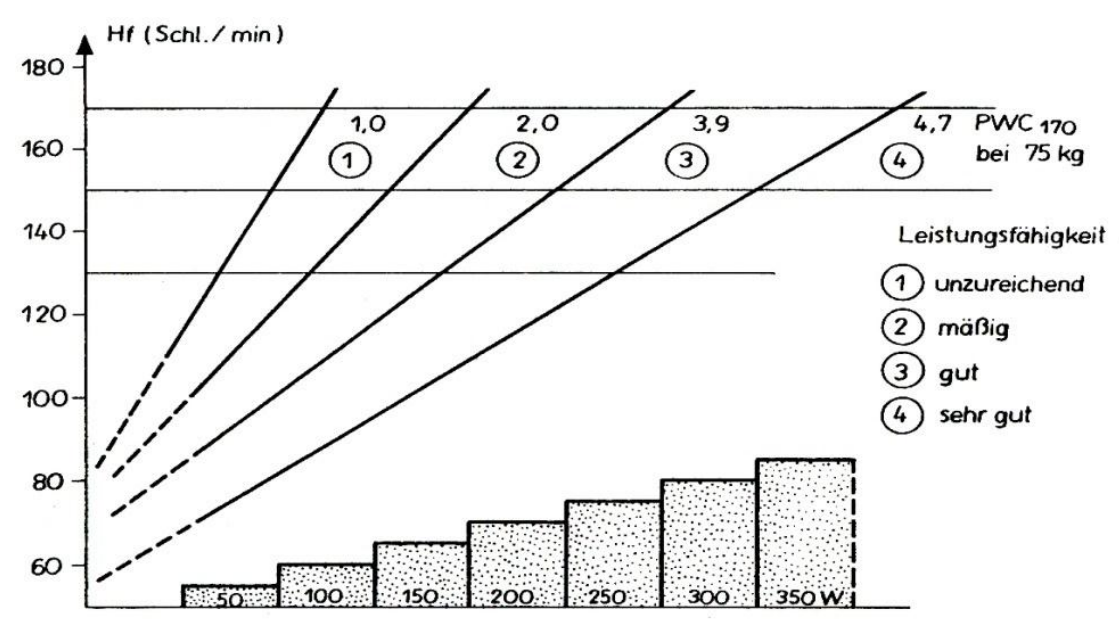

Bewertung der Leistungsfähigkeit bei unterschiedlich erreichten Wattstufen, bzw. umgerechnet in Watt/Kg Körpermasse (Hofmann \& Kalj, 2004).

Der PWC-Test startet bei 25 Watt für drei Minuten. Nun wird alle Drei Minuten um 25 Watt gesteigert, bis eine Herzfrequenz von 110, 130, 150 oder 170 Schlägen/min. (je nach Testart PWC110, PWC130, PWC150 oder PWC170) erreicht wird. Nachdem die Zielherzfrequenz erreicht wurde, wird bei einer selbstgewählten Belastungsstufe (in der vorliegenden Studie lag diese zwischen 30 und Watt) noch 4 Minuten ein Cool-Down gefahren. 
Anlage 8: Belastungsgestaltung und Trainingsprinzipien

Training ist ganz allgemein ein Sammelbegriff aller Maßnahmen des Prozesses zur Steigerung, Stabilisierung und teilweise auch Reduzierung der sportlichen Leistung. Training ist im biologischen Sinne ein anpassendes Reagieren menschlicher Systeme (z.B. Herz-Kreislauf-System, Muskelsystem, Zentralnervensystem, hormonelles System) nach einer Ursachen-Wirkungs-Kette. Zentrale Bedeutungen in dieser Kette haben Trainingsbelastung, Trainingsbeanspruchung, und Trainingsanpassung.

Trainingsbelastung: Gesamtheit auf den Körper einwirkende Belastungsreize. Gewöhnlich wird zwischen äußerer und innerer Belastung unterschieden.

Trainingsanpassung: Die funktionelle und morphologische Veränderung der Organsysteme auf die wirksamen Belastungsreize. Die Anpassung äußert sich für gewöhnlich zweifach: in einer Vergrößerung der Leistungsreserven und in der Fähigkeit zu tiefer Ausschöpfung dieser Reserven.

Belastungsintensität: Stärke des Belastungsreizes. Anstrengungsgrad, mit dem eine Übung durchgeführt wird. Sie wird in Zeiten, Geschwindigkeiten, Lasten oder physiologischen Parametern erfasst.

Belastungsdauer: Zeitdauer eines Einzelreizes oder einer Übungsserie. Sie wird durch Zeitangaben oder durch Wiederholungszahlen erfasst.

Belastungsdichte: Zeitspanne zwischen den einzelnen Belastungsreizen, mit der der Wechsel zwischen Belastung und Erholung reguliert wird.

Belastungsumfang: Gesamtmenge an Belastungsreizen in einer Trainingseinheit oder auch über längere Trainingsabschnitte. Trainingshäufigkeit: Anzahl der Trainingseinheiten, gemessen in Tagen (Grosser, Harischka, \& Zimmermann, 2008). 
Gleichgewichtsfähigkeit: Eine durch Lernen erworbene generalisierbare Fähigkeit, um den Körper unter allen Bedingungen im Gleichgewicht zuhalten oder es wiederherzustellen, als auch die Bewegungshandlung unter den verschiedensten Bedingungen ausführen zu können.

Kinästhetische Differenzierungsfähigkeit: Sie befähigt eine Person, Teil- und Ganzkörperbewegungen auszuführen, wobei die nacheinander folgenden Bewegungsabschnitte fließend ineinander übergehen, aber dennoch gut sichtbar voneinander ausgeführt werden.

Rhythmusfähigkeit: Die Rhythmusfähigkeit beruht entweder auf der Generierung eines eigenen, internen Bewegungsrhythmus oder auf der fixen oder auch variablen Synchronisation der Bewegungsabläufe mit rhythmischen externen Signalen.

Räumliche Orientierungsfähigkeit: Die Orientierungsfähigkeit sorgt für die Wahrnehmung der Position und der Bewegung der Person auf einer Fläche oder im Raum.

Reaktionsfähigkeit: Die Reaktionsfähigkeit zeichnet sich durch zweckgebundene und zielgerichtete sensomotorische Handlungen auf verschiedene externe Signale aus.

Umstellungsfähigkeit: Die Umstellungsfähigkeit sorgt für das situative Modifizieren bis hin zur völligen Neuentscheidung und damit den Ersatz einer laufenden Bewegungshandlung durch eine andere.

Kopplungsfähigkeit: Die Kopplungsfähigkeit führt dazu, Teilkörperbewegungen aufeinander abzustimmen und im zeitlichen Nacheinander der Gesamtbewegung zu unterstellen (Bertram \& Laube, 2008).

Ein rehabilitatives Training zielt besonders auf eine Verbesserung der oben genannten Fertigkeiten ab, da die Adaptationsprozesse sich in einem Zeitraum von 3-4 Wochen abspielen. 
Anlage 10: Die konditionellen Fähigkeiten (fünf motorischen Hauptbeanspruchungsformen)

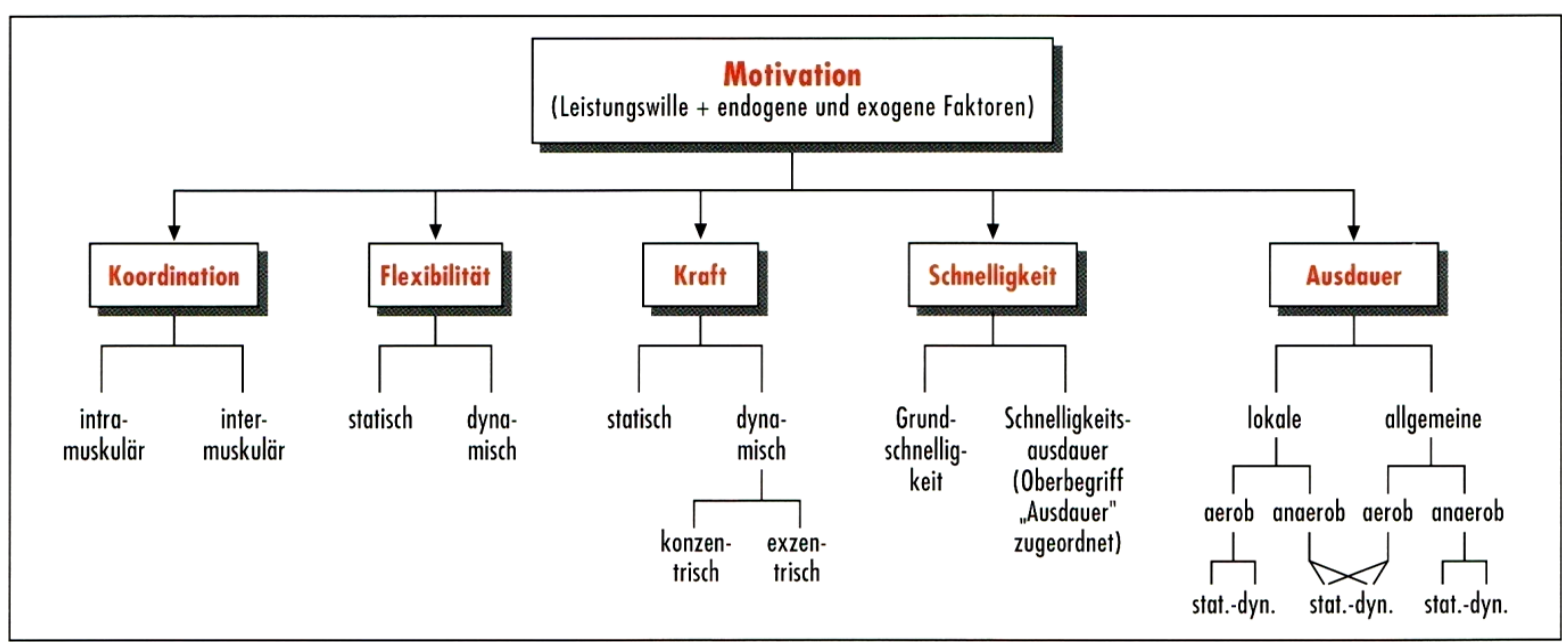

In der vorliegenden Studie waren besonders die Koordination und die statische Kraft von entscheidender Bedeutung. Diese Fähigkeiten hatten auf die Kraftmessungen mit der Kraftmessdose den größten Einfluss. Durch Verbesserung der inter- und Intramuskulären Koordination ist es der Patientin später möglich gewesen, eine größere statische Kraft zu generieren. Die Motivation als übergeordneter Faktor hat einen wesentlichen Einfluss auf die Reproduzierbarkeit der Messungen (Hollmann \& Hettinger). 


\section{Eidesstattliche Erklärung:}

„Ich versichere, dass ich die eingereichte Dissertation „Evaluation über die Effektivität eines strukturierten Trainingsprogramms zur Behandlung des chronischen FatigueSyndroms bei Brustkrebspatientinnen" selbständig und ohne unerlaubte Hilfsmittel verfasst habe. Anderer als der von mir angegebenen Hilfsmittel und Schriften habe ich mich nicht bedient. Alle wörtlich oder sinngemäß den Schriften anderer Autoren entnommenen Stellen habe ich kenntlich gemacht."

\section{Lebenslauf:}

Name: Marcus Wuttke

Familienstand: verheiratet

Ausbildung:

Seit 2009 Wissenschaftlicher Mitarbeiter der Sportmedizin am Institut für Sportwissenschaften der Universität Göttingen

2005 - heute Gesundheitszentrum Promotio in Göttingen, als Physiotherapeut

2007 - 2009 Doktorand der Sportwissenschaften am Institut für Sportwissenschaft der Universität Göttingen

2005 - 2007 Universitätsklinikum Göttingen, Abt. Sportmedizin sowie klinische und experimentelle Endokrinologie als wissenschaftlicher Mitarbeiter in diversen Forschungsprojekten

2004 - 2005 Masterstudiengang am Trinity College in Dublin, Irland mit Abschluss Master of Science in Sports Medicine

2001 - 2004 Ausbildung zum Physiotherapeuten an der Medizinischen Akademie in Seesen

1999 - 2001 Studium im Fachbereich Physiktechnik an der Fachhochschule Hildesheim/ Holzminden ohne Abschluss

1997 - 1998 Zivildienst beim ASC Göttingen (Sportverein)

1997 - 1997 Befristeter Arbeitsvertrag Feinmechaniker (Feingerätebau), Max Planck Institut für biophysikalische Chemie in Göttingen

1994 - 1997 Ausbildung zum Feinmechaniker (Feingerätebau), Max Planck Institut für biophysikalische Chemie in Göttingen 
